Configurações das Linhas de Curvatura Principal sobre Superfícies Seccionalmente Suaves

Gláucia Aparecida Soares Miranda

TESE APRESENTADA

$\mathrm{AO}$

Instituto de Matemática e Estatística

DA

Universidade de SÃo Paulo

PARA

OBTENÇÃO DO TÍTULO

$\mathrm{DE}$

Doutor EM CIÊNCIAS

\author{
Programa: Matemática \\ Orientador: Prof. Dr. Jorge Manuel Sotomayor Tello
}

Projeto financiado pela CAPES

São Paulo, agosto de 2014 


\title{
Configurações das Linhas de Curvatura Principal sobre Superfícies Seccionalmente Suaves
}

\begin{abstract}
Esta versão da tese contém as correções e alterações sugeridas pela Comissão Julgadora durante a defesa da versão original do trabalho, realizada em 26/06/2014. Uma cópia da versão original está disponível no Instituto de Matemática e Estatística da Universidade de São Paulo.
\end{abstract}

Comissão Julgadora:

- Prof. Dr. Jorge Manuel Sotomayor Tello (IME - USP)

- Prof. Dr. Ronaldo Alves Garcia (UFG)

- Prof. Dr. Luis Fernando de Osório Mello (UNIFEI)

- Prof. Dr. Fábio Scalco Dias (UNIFEI)

- Prof. Dra. Martha Patrícia Dussan Angulo (IME - USP) 


\section{Dedicatória}

Ao meu pai,

Geraldo Saraiva Soares. 


\section{Agradecimentos}

A Deus, por tudo.

Ao meu orientador, professor Jorge Manuel Sotomayor Tello pelos ensinamentos, paciência, disponibilidade (até nos finais de semana) e atenção dedicados à realização deste trabalho.

Ao professor Ronaldo Alves Garcia, pelas valiosas contribuições dadas desde a fase inicial do projeto e por receber-me, tantas vezes, para discutirmos pontos importantes desta tese.

Aos demais membros da banca, Luis Fernando de Osório Mello, Fábio Scalco Dias e Martha Patrícia Dussan Angulo, pelas sugestões.

À minha família e aos meus amigos pelo apoio.

Ao meu marido Marcelo, pelo apoio e paciência durante todos esses anos.

À CAPES pelo apoio financeiro indispensável. 


\title{
Resumo
}

\author{
SOARES MIRANDA, G.A. Configurações das Linhas de Curvatura Principal \\ sobre Superfícies Seccionalmente Suaves. 2014. 122 f. Tese (Doutorado) - Insti- \\ tuto de Matemática e Estatística, Universidade de São Paulo, São Paulo, 2014.
}

Nesta tese apresentamos uma contribuição para o estudo da transição do retrato de fase de uma equação diferencial descontínua específica ao longo de uma linha de descontinuidade. A equação diferencial que tratamos neste trabalho é a das linhas de curvatura principal de uma superfície $\mathbf{S}$ contendo uma curva distinguida $\mathbf{B}$ e imersa em $\mathbb{R}^{3}$. A linha de descontinuidade é a curva $\mathbf{B}$, a qual é o bordo comum de duas superfícies suaves justapostas que formam $\mathbf{S}$.

$\mathrm{Na}$ primeira parte do trabalho consideramos a superfície seccionalmente suave, $\mathbf{S}=\mathbf{S}^{+} \cup \mathbf{B} \cup \mathbf{S}^{-}$, obtida pela justaposição de $\mathbf{S}^{+}$e $\mathbf{S}^{-}$ao longo do bordo comum $\mathbf{B}$. O estudo da configuração principal de $\mathbf{S}$ nos casos em que as linhas de curvatura principal das superfícies $\mathbf{S}^{+}$e $\mathbf{S}^{-}$tem contato quadrático ou cruzam transversalmente $\mathbf{B}$ foi feito por comparação com a configuração principal de uma superfície suave $\mathbf{S}_{\varepsilon}$, obtida de $\mathbf{S}$ pelo processo da $\varepsilon$-regularização ao longo da curva de descontinuidade $\mathbf{B}$, com $\varepsilon>0$ suficientemente pequeno.

Na segunda parte do trabalho estudamos as linhas de curvatura principal de uma superfície $\mathbf{S}$ em $\mathbb{R}^{3}$ com bordo $\mathbf{B}$ e da superfície suave obtida de $\mathbf{S}$ através dos processos de engrossamento e regularização definidos por Garcia e Sotomayor em [5], onde os autores consideraram o caso genérico, sem pontos umbílicos e contato quadrático de uma linha de curvatura principal com B. Damos aqui continuidade ao estudo feito em [5] analisando o caso de contato cúbico com o bordo $\mathbf{B}$.

Obtivemos que dos pontos da curva bordo comum B de contato quadrático e de cruzamento transversal emergem, sobre a superfície regularizada, pontos umbílicos Darbouxianos dos tipos $D_{1}$ e $D_{3}$, enquanto que, para o ponto sobre $\mathbf{B}$ de contato cúbico obtivemos pontos umbílicos Darbouxianos dos tipos $D_{1}, D_{2}$ e $D_{3}$ e também pontos umbílicos não Darbouxianos dos tipos $D_{12}$ e $D_{23}$.

Palavras chave: singularidade quadrática; cruzamento transversal; singularidade cúbica; pontos umbílicos; configuração principal. 


\section{Abstract}

\section{SOARES MIRANDA, G.A. Configurations of Principal Curvature Lines on}

Piecewise Smooth Surfaces. 2014. 122 p. Tese (Doutorado) - Instituto de Matemática e Estatística, Universidade de São Paulo, São Paulo, 2014.

In this work we present a contribution to the study of the transition of the phase portrait of a specific discontinuous differential equation along a line of discontinuity. The differential equations under consideration will be that of the principal curvature lines of a surface $\mathbf{S}$ with a distinguished curve $\mathbf{B}$ immersed in $\mathbb{R}^{3}$, where the line of discontinuity is the curve $\mathbf{B}$ which is the common border of two smooth surfaces attached to make up $\mathbf{S}$.

In the first part of the work we consider a piecewise smooth surface $\mathbf{S}=\mathbf{S}^{+} \cup \mathbf{B} \cup \mathbf{S}^{-}$, obtained by the juxtaposition of two smooth surfaces $\mathbf{S}^{+}$and $\mathbf{S}^{-}$along their common border $\mathbf{B}$. The analysis of the principal configuration of $\mathbf{S}$ in the cases where the principal curvature lines of the surfaces $\mathbf{S}^{+}$and $\mathbf{S}^{-}$have quadratic contact or cross transversally $\mathbf{B}$ was carried out by comparison with a smooth surface $\mathbf{S}_{\varepsilon}$, obtained from $\mathbf{S}$ by the $\varepsilon$-regularization along the discontinuity curve $\mathbf{B}$, with small $\varepsilon>0$.

In the second part of the work we study the principal curvature lines of a surface $\mathbf{S}$ in $\mathbb{R}^{3}$ with boundary $\mathbf{B}$ and of the smooth surface obtained from $\mathbf{S}$ by thickening and smoothing introduced by Garcia and Sotomayor in [5], where they considered the generic case of no umbilic points and at most quadratic contact of principal lines with $\mathbf{B}$. Here we pursue the study in [5] and analyze the case of cubic contact with the border $\mathbf{B}$.

We established that while from quadratic contact points with $\mathbf{B}$ emerge on the smoothed surface Darbouxian umbilics of $D_{1}$ and $D_{3}$ types, from the cubic contact points appear Darbouxian umbilics of types $D_{1}, D_{2}$ and $D_{3}$ as well as non Darbouxian points of types $D_{12}$ and $D_{23}$.

Keywords: quadratic singularity; cross transversally; cubic singularity; umbilic points; principal configuration. 


\section{Sumário}

Lista de Símbolos

Lista de Figuras vii

Introdução xi xi

1 Levantamento de Lie-Cartan, Pontos Umbílicos e suas Bifurcações 1

1.1 Equações Diferenciais das Linhas de Curvatura Principal . . . . . . . . . . 1

1.2 O Campo de Lie-Cartan e os Pontos Umbílicos Darbouxianos . . . . . . . 3

1.3 Pontos Umbílicos de Codimensão 1 . . . . . . . . . . . . . . . . . . . 7

1.3 .1 O Padrão de Bifurcação do Ponto Umbílico $D_{12}$. . . . . . . . . . . 7

$1.3 .2 \quad$ O Padrão de Bifurcação do Ponto Umbílico $D_{23}$. . . . . . . . . . . . 10

2 Configuração Principal sobre Superfícies Suaves por Partes e Curva de

Descontinuidade Plana

2.1 Configuração das Linhas de Curvatura Principal de uma Superfície Suave por Partes . . . . . . . . . . . . . . . . . . . . . . . . 13

$2.1 .1 \quad 1^{\circ}$ Caso: Singularidade Quadrática . . . . . . . . . . . . . . . 14

$2.1 .2 \quad 2^{\circ}$ Caso: Ponto de Cruzamento Transversal . . . . . . . . . . . . . . 23

$2.1 .3 \quad 3^{\circ}$ Caso: Ponto de Dobra . . . . . . . . . . . . . . . . . . . . . 32

2.2 Regularização da Superfície S em uma Carta Local . . . . . . . . . . . . . 32

$2.2 .1 \quad 1^{\circ}$ Caso: Regularização na Vizinhança de uma Singularidade Quadrática $\left(k_{3}^{+}=k_{3}^{-}=0\right)$. . . . . . . . . . . . 38

$2.2 .2 \quad 2^{\circ}$ Caso: Regularização na Vizinhança de um Ponto de Cruzamento Transversal $\left.\left(k_{3}^{+} \cdot k_{3}^{-} \neq 0\right)\right] \ldots \ldots \ldots . \ldots \ldots 4$

$2.2 .3 \quad 3^{\circ}$ Caso: Regularização na Vizinhança de um Ponto de Dobra

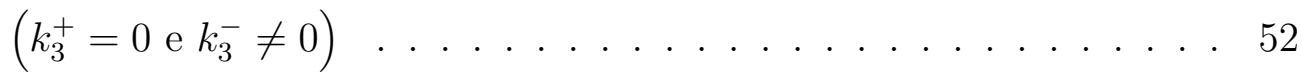

3 Configuração Principal sobre Superfícies Suaves por Partes e Curva de Descontinuidade com Torção $\quad 55$

3.1 Configuração Principal da Superfície $\mathbf{S}=\mathbf{S}^{+} \cup \mathbf{B} \cup \mathbf{S}^{-}$: Singularidades Quadráticas e Pontos Umbílicos . . . . . . . . . . . . . . 56 
3.2 Regularização da Superfície $\mathbf{S}$. . . . . . . . . . . . . . . . . . . . . 62

3.3 Pontos de Dobras e Cruzamentos Transversais . . . . . . . . . . . . . . . 68

3.3 .1 Ponto de Dobra . . . . . . . . . . . . . . . . . . . . . 68

3.3 .2 Cruzamentos Transversais . . . . . . . . . . . . . . . . . . 68

4 Configuração Principal sobre Superfícies com Bordo em $\mathbb{R}^{3}$ : Contato $\begin{array}{ll}\text { Cúbico e Pontos Umbílicos } & 73\end{array}$

4.1 Introdução . . . . . . . . . . . . . . . . . . . . . . . . 73

$4.1 .1 \quad$ Envelopes de Superfícies Regulares _. . . . . . . . . . . . . 74

4.2 Configuração Principal de $\mathbf{S}_{\varepsilon}=\mathbf{S}_{\varepsilon,+} \cup \mathbf{T}_{\varepsilon} \cup \mathbf{S}_{\varepsilon,-} \ldots \ldots \ldots \ldots 75$

4.3 Regularização de $\mathbf{S}_{\varepsilon}$ em uma Carta Local . . . . . . . . . . . . . . . . 83

4.4 Apêndice. . . . . . . . . . . . . . . . . . . . . . . . . . . . . . . 104

\begin{tabular}{ll}
\hline Conclusão & 107
\end{tabular}

\begin{tabular}{ll}
\hline Referências Bibliográficas & 109
\end{tabular}

Índice Remissivo 111 


\section{Lista de Figuras}

$1.1 \quad$ Linhas de curvaturas principais próximas aos pontos umbílicos $D_{i}$ e suas

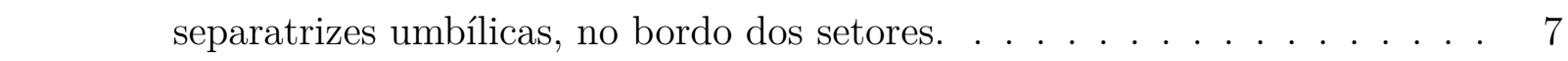

1.2 Ponto umbílico $D_{12}$ e sua bifurcação. . . . . . . . . . . . . . . . . . . . . . 10

1.3 Bifurcação do ponto umbílico $D_{23}$. Os pontos umbílicos $D_{2}$ e $D_{3}$ colidem

formando o ponto $D_{23}$, e depois não há pontos umbílicos. Fixada uma

\begin{tabular}{|c|}
\hline orientação, as linhas tracejadas (respectivamente contínuas) ilustram as \\
\hline \hline linhar
\end{tabular}

linhas de curvatura principal da folheação principal mínima, $\mathcal{F}_{1, \alpha}$ (respectivamente máxima, $\left.\mathcal{F}_{2, \alpha}\right) . \ldots \ldots \ldots \ldots$. . . . . . . . . . . . 11

2.1 Configuração principal de $\mathbf{S}^{+}$em uma vizinhança da origem: tangência \begin{tabular}{|c|c|}
\hline interna para $c>0$ e externa para $c<0$. Supondo-se $k_{2}^{+}<k_{1}$, temos \\
\hline
\end{tabular} \begin{tabular}{|c|}
\hline que as linhas tracejadas (respectivamente contínuas) ilustram as linhas de \\
\hline
\end{tabular} curvatura principal mínima (respectivamente máxima). . . . . . . . . . . . 18

2.2 Configuração principal de $\mathbf{S}^{-}$em uma vizinhança da origem: tangência \begin{tabular}{|c|c|}
\hline interna para $\gamma>0$ e externa para $\gamma<0$. Supondo-se $k_{1}<k_{2}^{-}$, temos \\
\hline
\end{tabular} \begin{tabular}{|c|}
\hline que as linhas tracejadas (respectivamente contínuas) ilustram as linhas de \\
\hline
\end{tabular}

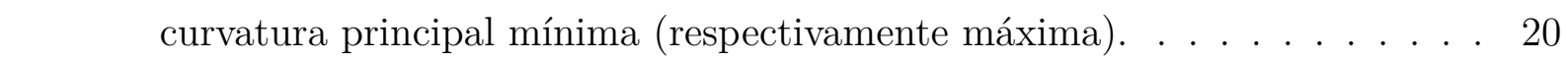

2.3 Configurações principais suaves por partes de $\mathbf{S}=\mathbf{S}^{+} \cup \boldsymbol{\delta} \cup \mathbf{S}^{-}$em uma vizinhança da origem considerando $k_{2}^{+}<k_{1}<k_{2}^{-}$. Linhas tracejadas (respectivamente contínuas) ilustram as linhas de curvatura principal mínima (respectivamente máxima). . . . . . . . . . . . . . . . 22

2.4 Configurações principais suaves por partes de $\mathbf{S}=\mathbf{S}^{+} \cup \boldsymbol{\delta} \cup \mathbf{S}^{-}$em uma vizinhança da origem considerando $k_{1}<k_{2}^{+}$e $k_{1}<k_{2}^{-}$. Linhas tracejadas (respectivamente contínuas) ilustram as linhas de curvatura principal mínima (respectivamente máxima). . . . . . . . . . . . . . . . 23

$2.5 \quad \delta\left(u_{0}\right)$ é um ponto de deslizamento para as linhas de curvatura principais

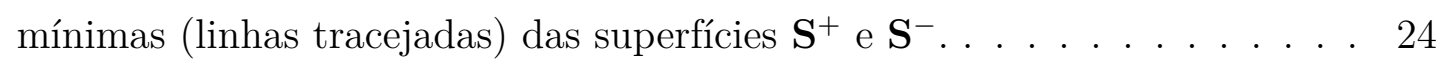

$2.6 \quad \delta\left(u_{0}\right)$ é um ponto de costura para as linhas de curvatura principais mínimas (linhas tracejadas) das superfícies $\mathbf{S}^{+}$e $\mathbf{S}^{-}$. . . . . . . . . . . . . 24 
$2.7 \delta\left(u_{0}\right)$ é um ponto de transição para as linhas de curvatura principais mínimas (linhas tracejadas) e máximas (linhas contínuas) das superfícies $\mathbf{S}^{+}$e

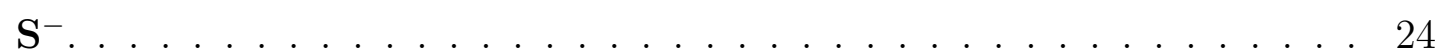

$2.8 \delta\left(u_{0}\right)$ é um ponto de costura para as linhas de curvatura principais mínimas

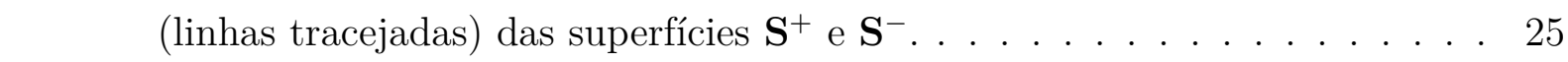

2.9 Exemplos de ângulos formados entre as linhas principais mínimas (linhas tracejadas) e as linhas principais máximas (linhas contínuas) de $\mathbf{S}^{+}$e de

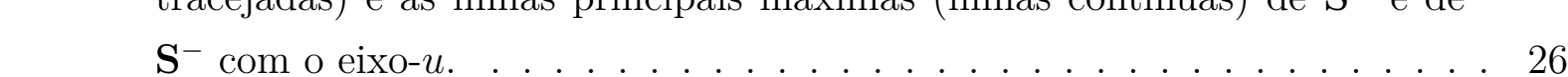

2.10 A origem $\delta(0)$ é um ponto de transição e, para as linhas de curvatura principal mínima, $\delta(u)$ é ponto de costura se $u>0$ e ponto de deslizamento se $u<0$. Linhas tracejadas (respectivamente contínuas) ilustram as linhas de curvatura principal mínima (respectivamente máxima). . . . . . . . . . 30

2.11 Configuração principal suave por partes de $\mathbf{S}=\mathbf{S}^{+} \cup \boldsymbol{\delta} \cup \mathbf{S}^{-}$em uma vizinhança de um ponto de $\boldsymbol{\delta}$-dobra, considerando $k_{2}^{+}<k_{1}$. Linhas tracejadas \begin{tabular}{|c|}
\hline (respectivamente contínuas) ilustram as linhas de curvatura principal mí- \\
\hline
\end{tabular}

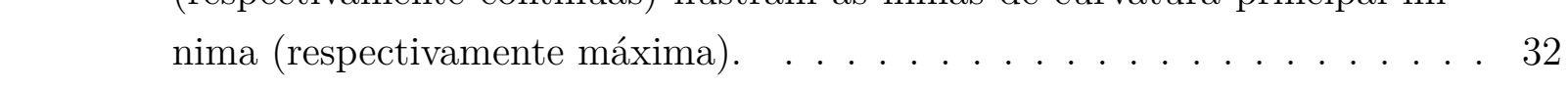

2.12 Gráfico de uma função de transição $\phi . \ldots . . . . . . .33$

2.13 Gráficos da função de transição $\phi_{\varepsilon}$, para $\varepsilon \in\left\{\varepsilon_{0}, \varepsilon_{1}\right\}, \varepsilon_{1}<\varepsilon_{0}$. . . . . . . . 34

2.14 À esquerda, temos que a origem é um ponto de tangência quadrática tal

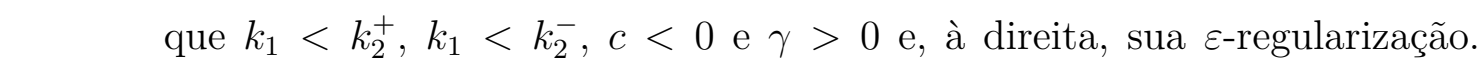
Linhas principais mínimas (linhas tracejadas) são deslizantes e as linhas

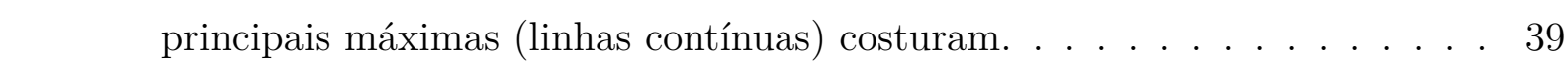

2.15 À esquerda, temos que a origem é um ponto de tangência quadrática tal

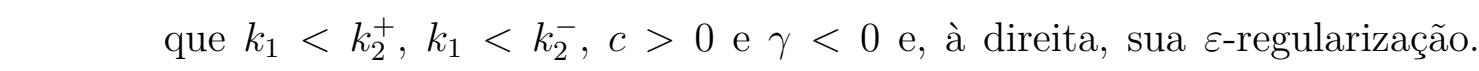
Linhas principais mínimas (linhas tracejadas) são deslizantes e as linhas

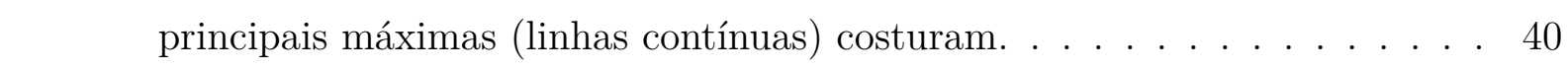

2.16 Regiões do plano $c \gamma$ onde temos uma única raiz real $(\Delta>0)$ ou três raízes reais distintas $(\Delta<0)$. . . . . . . . . . . . . . . . . . . . . . . . . 43

2.17 À esquerda, temos que a origem é um ponto de tangência tal que $k_{2}^{+}<$ $k_{1}<k_{2}^{-}, c>0, \gamma>0$ e $\gamma>c\left(\frac{k_{1}-k_{2}^{-}}{k_{1}-k_{2}^{+}}\right)$e, à direita, sua $\varepsilon$-regularização. Linhas principais mínimas (linhas tracejadas) são deslizantes e as linhas principais máximas (linhas contínuas) costuram. . . . . . . . . . . . . . . 46

2.18 À esquerda, temos que a origem é um ponto de tangência tal que $k_{2}^{+}<$ $k_{1}<k_{2}^{-}, c>0, \gamma>0$ e $\gamma<c\left(\frac{k_{1}-k_{2}^{-}}{k_{1}-k_{2}^{+}}\right)$e, à direita, sua $\varepsilon$-regularização. Linhas principais máximas (linhas contínuas) são deslizantes e as linhas principais mínimas (linhas tracejadas) costuram. . . . . . . . . . . . . . . 46 
2.19 À esquerda, temos que a origem é um ponto de cruzamento transversal (observe que devemos ter $\omega=90^{\circ}$ ) e, à direita, sua $\varepsilon$-regularização. Linhas principais mínimas (linhas tracejadas) são deslizantes se $u<0$ e costuram \begin{tabular}{|c|c|}
\hline se $u>0$. As linhas principais máximas (linhas contínuas) costuram se \\
\hline
\end{tabular}

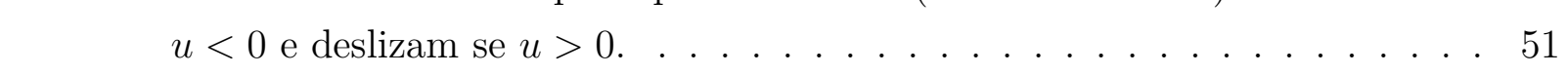

2.20 À esquerda, temos que a origem é um ponto de $\boldsymbol{\delta}$-dobra $\left(k_{3}^{+}=0, k_{3}^{-} \neq 0\right.$ e $\left.c>0\right)$ e, à direita, sua $\varepsilon$-regularização. . . . . . . . . . . . . . . . . . . 53

2.21 À esquerda, temos que a origem é um ponto de $\boldsymbol{\delta}$-dobra $\left(k_{3}^{+}=0, k_{3}^{-} \neq 0\right.$ e $\left.c<0\right)$ e, à direita, sua $\varepsilon$-regularização. . . . . . . . . . . . . . . . . . . . 53

3.1 Configuração principal de $\mathbf{S}^{+}$em uma vizinhança de $\delta\left(u_{0}\right)$ : tangência interna para $\tau_{g}^{\prime}\left(u_{0}\right)>0$ e externa para $\tau_{g}^{\prime}\left(u_{0}\right)<0$, considerando $u_{0}=0$. Supondo $\left(k_{n}-k^{+}\right)\left(u_{0}\right)>0$, temos que as linhas tracejadas (respectivamente contínuas) ilustram as linhas de curvatura principal mínima (respectiva-

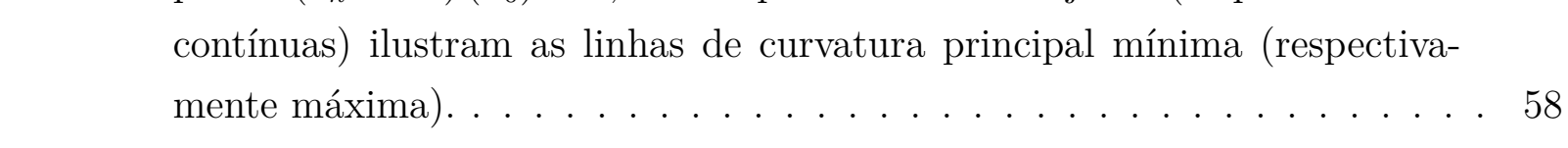

3.2 Configuração principal de $\mathbf{S}^{-}$em uma vizinhança da origem: tangência in\begin{tabular}{|c|}
\hline terna para $\tau_{g}^{\prime}\left(u_{0}\right)>0$ e externa para $\tau_{g}^{\prime}\left(u_{0}\right)<0$, considerando $u_{0}=0$. Su- \\
\hline \hline pondo $\left(k_{n}-k^{-}\right)\left(u_{0}\right)<0$, temos que as linhas tracejadas (respectivamente \\
\hline contínuas) ilustram as linhas de curvatura principal mínimas (respectiva- \\
\hline
\end{tabular}

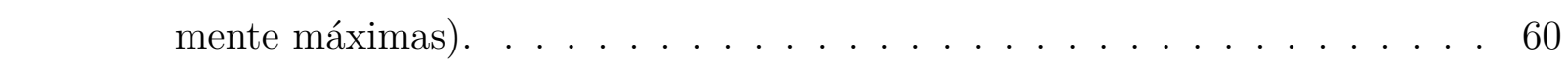

3.3 Configuração principal suave por partes de $\mathbf{S}=\mathbf{S}^{+} \cup \mathbf{B} \cup \mathbf{S}^{-}$em uma vizinhança da origem considerando $u_{0}=0$ e $k^{+}\left(u_{0}\right)<k_{n}\left(u_{0}\right)<k^{-}\left(u_{0}\right)$. Linhas tracejadas (respectivamente contínuas) ilustram as linhas de curvatura principal mínimas (respectivamente máximas). Dois casos: $\left.\tau_{g}^{\prime}\left(u_{0}\right)\right)>0$, à

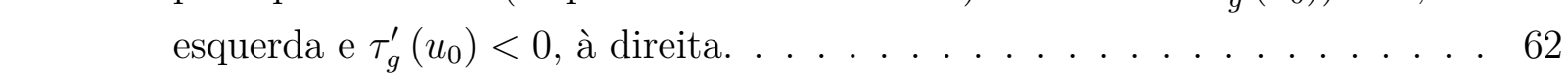

3.4 Configuração principal suave por partes de $\mathbf{S}=\mathbf{S}^{+} \cup \mathbf{B} \cup \mathbf{S}^{-}$em uma vizinhança da origem considerando $u_{0}=0$ e $k_{n}\left(u_{0}\right)<k^{+}\left(u_{0}\right)$ e $k_{n}\left(u_{0}\right)<$ $k^{-}\left(u_{0}\right)$. Linhas tracejadas (respectivamente contínuas) ilustram as linhas de curvatura principal mínimas (respectivamente máximas). Dois casos: $\tau_{g}^{\prime}\left(u_{0}\right)>0$, à esquerda e $\tau_{g}^{\prime}\left(u_{0}\right)<0$, à direita. . . . . . . . . . . . 62

3.5 Exemplos de ângulos formados entre as linhas principais mínimas (linhas tracejadas) e as linhas principais máximas (linhas contínuas) de $\mathbf{S}^{+}$e de

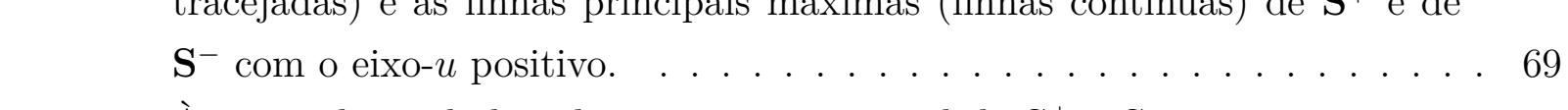

$3.6 \quad \grave{A}$ esquerda, as linhas de curvatura principal de $\mathbf{S}^{+}$e $\mathbf{S}^{-}$são transversais ao bordo comum $\mathbf{B}$ e, à direita, a $\varepsilon$-regularização de $\mathbf{S}$. . . . . . . . . . . . . 72

4.1 Um aberto do $\varepsilon$-engrossamento da superfície $\mathbf{S}$. . . . . . . . . . . . . . 75 
4.2 Configurações suaves por partes da superfície $\mathbf{S}_{\varepsilon}=\mathbf{S}_{\varepsilon,+} \cup \mathbf{T}_{\varepsilon} \cup \mathbf{S}_{\varepsilon,-}$ em uma vizinhança do ponto de tangência quadrática, sendo $k_{n}\left(u_{0}\right)<k_{n}^{\perp}\left(u_{0}\right)$. Dois casos: tangência interna se $\tau_{g}^{\prime}\left(u_{0}\right)>0$, à esquerda, e tangência interna se $\tau_{g}^{\prime}\left(u_{0}\right)<0$, à direita. Para mais detalhes sobre o caso quadrático ver [5].] . 82

4.3 Configurações suaves por partes da superfície $\mathbf{S}_{\varepsilon}=\mathbf{S}_{\varepsilon,+} \cup \mathbf{T}_{\varepsilon} \cup \mathbf{S}_{\varepsilon,-}$ em uma vizinhança do ponto de contato cúbico, sendo $k_{n}\left(u_{0}\right)<k_{n}^{\perp}\left(u_{0}\right)$. Dois casos: $\tau_{g}^{\prime \prime}\left(u_{0}\right)>0$, à esquerda e $\tau_{g}^{\prime \prime}\left(u_{0}\right)<0$, à direita. . . . . . . . . . . . . 83

4.4 Configurações suaves por partes da superfície $\mathbf{S}_{\varepsilon}=\mathbf{S}_{\varepsilon,+} \cup \mathbf{T}_{\varepsilon} \cup \mathbf{S}_{\varepsilon,-}$ em uma vizinhança do ponto de contato cúbico, sendo $k_{n}\left(u_{0}\right)>k_{n}^{\perp}\left(u_{0}\right)$. Dois casos: $\tau_{g}^{\prime \prime}\left(u_{0}\right)>0$, à esquerda e $\tau_{g}^{\prime \prime}\left(u_{0}\right)<0$, à direita. . . . . . . . . . . . . 83

4.5 B é o deslocamento paralelo do bordo $\mathbf{B}_{\varepsilon,+}$ na direção de $-\mathcal{N}$, sendo $\mathcal{N}$ campo normal da superfície $\mathbf{S}_{\varepsilon,+} \ldots \ldots \ldots \ldots$. . . . . . . . . . . 85

4.6 Solução do sistema (4.14): para cada $u_{0}$ fixado temos um círculo de raio $f\left(u_{0}, v\right)$ obtido da interseção entre a esfera de centro $Q=\delta_{+}\left(u_{0}\right)-\varepsilon \mathcal{N}\left(u_{0}\right)$ e raio $\varepsilon$ e o plano $\Pi$ de equação $\left(1+\varepsilon k_{n}\left(u_{0}\right)\right) x+\varepsilon \tau_{g}\left(u_{0}\right) y=0$. . . . . . . . 86

$4.7 \quad$ Família a 1 parâmetro de superfícies $\mathbf{S}_{\tau_{0}}$ com bordo variável $\mathbf{B}_{\tau_{0}}$. Se $\tau_{0}=0$ \begin{tabular}{|c|c|c|}
\hline temos um ponto de contato cúbico entre as linhas de curvatura principal \\
\hline
\end{tabular} mínima de $\mathbf{S}_{0}=\mathbf{S}$ com o bordo $\mathbf{B}_{0}=\mathbf{B}$. Com relação à superfície $\mathbf{S}_{\tau_{0}}$ $\left(\tau_{0}<0\right)$, o contato das linhas de curvatura de $\mathbf{S}_{\tau_{0}}$ com $\mathbf{B}_{\tau_{0}}$ é quadrático externo (curva azul) e quadrático interno (curva vermelha). Destes pontos de tangências quadráticas externa e interna emergem pontos umbílicos Darbouxianos, respectivamente, dos tipos $D_{1}$ e $D_{3}$; isto foi demonstrado por \begin{tabular}{|c|}
\hline Garcia e Sotomayor em [5], em um contexto local específico e para valores \\
\hline
\end{tabular} pequenos do parâmetro $\varepsilon>0 . \ldots \ldots . \ldots . \ldots . \ldots 92$

4.8 Diagrama de bifurcação em uma vizinhança de um ponto de contato cúbico. 93

4.9 Gráfico da função de transição $\phi$, para $a>0$ e $\mu>0$ suficientemente pequenos. . . . . . . . . . . . . . . . . . . 105 


\section{Introdução}

A estrutura formada pelos pontos umbílicos e pelas linhas de curvatura principal de uma superfície imersa em $\mathbb{R}^{3}$ definem o que chamamos de configuração principal da superfície. As linhas de curvatura principal são as curvas integrais dos campos de direções, segundo as quais a curvatura normal tem valores extremais: máximo ou mínimo. As curvaturas normais correspondentes são chamadas curvaturas principais e os pontos onde as curvaturas principais coincidem são os pontos umbílicos. As linhas de curvatura principal estão definidas em toda a superfície e interceptam-se ortogonalmente fora dos pontos umbílicos. Ver [6, 13].

O estudo da configuração principal de uma superfície em $\mathbb{R}^{3}$ mereceu a atenção inicial de matemáticos como Monge e Dupin. Em 1796, Monge em [10] apresentou a descrição, por integração direta das equações diferenciais das linhas de curvatura principal, do primeiro exemplo de configuração principal não trivial que ocorre no elipsóide de três eixos distintos

$$
\frac{x^{2}}{a^{2}}+\frac{y^{2}}{b^{2}}+\frac{z^{2}}{c^{2}}-1=0, \quad a>b>c>0 .
$$

O elipsóide, com sua configuração principal, é conhecido como o Elipsóide de Monge. Este é o primeiro exemplo encontrado na literatura de folheações com singularidades. Posteriormente, várias contribuições foram dadas por Dupin, Darboux e outros.

Em 1896, Darboux [2], descreveu o comportamento das linhas de curvatura principal próximas de um ponto umbílico genérico, na classe de superfícies analíticas. Sob certas condições nas terceiras derivadas da superfície, ele encontrou três tipos $D_{1}, D_{2}$ e $D_{3}$, conhecidos como pontos umbílicos Darbouxianos. Em [8] pode-se encontrar dados históricos sobre este assunto.

Em 1982, Gutierrez e Sotomayor [7, 8], abordaram o estudo da configuração principal de uma superfície sem bordo $\mathbf{S}$, do ponto de vista da estabilidade estrutural sob pequenas deformações da superfície $\mathbf{S}$. Eles estabeleceram que, com topologias adequadas, toda superfície compacta pode ser $C^{2}$ - aproximada por uma cuja configuração principal é estruturalmente estável. As condições suficientes para a estabilidade estrutural da configuração principal de uma superfície em $\mathbb{R}^{3}$, sem bordo e compacta são as seguintes:

(i) Todos os pontos umbílicos são Darbouxianos; 
(ii) Todos os ciclos principais são hiperbólicos;

(iii) Não existem conexões ou autoconexões de separatrizes umbílicas;

(iv) O conjunto limite de cada linha de curvatura principal está contido no conjunto dos pontos umbílicos ou ciclos principais.

Em 2002, Garcia e Sotomayor [5], deram um primeiro passo no sentido de estender, para superfícies $\mathbf{S}$ com bordo $\mathbf{B}$, as propriedades de estabilidade das configurações principais obtidas em [7, 8] para superfícies sem bordo. Outro tipo de deformação de (S, B) foi introduzida por estes autores em [5], onde o estudo da configuração principal de (S, B) foi feito por comparação com a configuração principal de uma deformação apropriada $\mathbf{S}_{\varepsilon, \mu}$, a qual foi obtida através dos processos de $\varepsilon$-engrossamento e $\mu$-regularização (ou $\mu$-suavização) aplicados à superfície com bordo.

Em linguagem geométrica, o $\varepsilon$-engrossamento de $\mathbf{S}$ é a fronteira de uma vizinhança tubular de $\mathbf{S}$ de raio $\varepsilon$, a qual consiste em duas cópias de $\mathbf{S}$, denotadas por $\mathbf{S}_{\varepsilon,+}$ e $\mathbf{S}_{\varepsilon,-}$, obtidas deslocando-se $\mathbf{S}$ de $\pm \varepsilon$ ao longo da sua normal e sendo $\mathbf{S}_{\varepsilon, \pm}$ unidas por um semitubo $\mathbf{T}_{\varepsilon}$, de raio $\varepsilon$ e com centro variando sobre o bordo $\mathbf{B}$ de $\mathbf{S}$. Veja definição 4.1.2. Estas três superfícies $C^{\infty}$, quando justapostas, formam uma superfície (dita suave por partes ou seccionalmente suave) que é só de classe $C^{1}$, ao longo dos bordos $\mathbf{B}_{\varepsilon, \pm}$ de $\mathbf{S}_{\varepsilon, \pm}$. A $\mu$-regularização consiste em considerar uma aproximação adicional para esta superfície por outra, de classe $C^{\infty}$, suportada numa faixa de largura $\mu$ ao longo dos bordos $\mathbf{B}_{\varepsilon, \pm}$.

Garcia e Sotomayor provaram que, com restrições específicas e das propriedades das funções suaves que caracterizam o processo, dos pontos de tangências supostas quadráticas das linhas de curvatura principal com o bordo $\mathbf{B}$ emergem pontos umbílicos Darbouxianos dos tipos $D_{1}$ e $D_{3}$ sobre $\mathbf{S}_{\varepsilon, \mu}$, para $\varepsilon$ e $\mu$ positivos e suficientemente pequenos. O tipo $D_{2}$ não aparece no processo de engrossamento e suavização da variedade com bordo definido pelos autores.

No presente trabalho propomos abordar uma situação mais geral de superfícies suaves por partes, acima discutidas, a partir do trabalho [5]. Na primeira parte da tese (capítulos 2 e 3) consideramos a superfície suave por partes $\mathbf{S}=\mathbf{S}^{+} \cup \mathbf{B} \cup \mathbf{S}^{-}$, obtida pela justaposição de duas superfícies suaves $\left(\mathbf{S}^{+}, \mathbf{B}\right)$ e $\left(\mathbf{S}^{-}, \mathbf{B}\right)$ ao longo do bordo comum $\mathbf{B}$ e estudamos os efeitos da regularização sobre os pontos de $\mathbf{B}$ de contato quadrático ou de cruzamento transversal. Na segunda parte do trabalho (capítulo 4) damos continuidade ao estudo iniciado em [5] por Garcia e Sotomayor, analisando um caso complementar, no qual o contato de uma linha de curvatura principal com o bordo $\mathbf{B}$ é cúbico, dito tangencialmente crítico. Apresentamos as condições para que destes pontos de contato quadrático, cruzamento transversal e de contato cúbico emerjam, sobre a superfície regularizada, pontos umbílicos.

A tese está divida da seguinte forma: 
No capítulo 1 fazemos uma revisão dos principais conceitos e resultados necessários para o bom desenvolvimento deste trabalho. A equação diferencial das linhas de curvatura de uma superfície imersa em $\mathbb{R}^{3}$ será apresentada. Também apresentamos as condições que definem os pontos umbílicos Darbouxianos dos tipos $D_{1}, D_{2}$ e $D_{3}$ e suas bifurcações de codimensão 1: os pontos $D_{12}$ e $D_{23}$.

No capítulo 2 consideramos a superfície seccionalmente suave $\mathbf{S}=\mathbf{S}^{+} \cup \boldsymbol{\delta} \cup \mathbf{S}^{-}$, obtida pela justaposição, ao longo de $\boldsymbol{\delta}$, de duas superfícies suaves $\mathbf{S}^{+}$e $\mathbf{S}^{-}$com bordo comum $\boldsymbol{\delta}$. Supomos que $\boldsymbol{\delta}$ é uma curva plana em $\mathbb{R}^{3}$ (ou seja, tem torção geodésica nula) e que $\mathbf{S}^{ \pm}$estão parametrizadas, sem perda de generalidade, por gráficos em uma vizinhança da origem. Definimos três tipos de pontos sobre o bordo comum $\boldsymbol{\delta}$ : pontos de contato quadrático, de dobra e de cruzamento transversal e estudamos os efeitos da regularização para estes três casos. Os principais resultados estão contidos nos teoremas 2.2.6, 2.2.10 e 2.2 .11 .

No capítulo 3 fizemos um estudo análogo ao do capítulo 2, considerando, no entanto, que a curva bordo comum tem torção genérica (possivelmente não nula). Nesse contexto, temos dois tipos de pontos a serem analisados: pontos de contato quadrático e de cruzamento transversal. Os principais resultados deste capítulo estão contidos nos teoremas 3.2 .4 e 3.3 .1

No capítulo 4 estudamos as linhas de curvatura principal de uma superfície $\mathbf{S}$ em $\mathbb{R}^{3}$ com bordo $\mathbf{B}$ e da superfície suave $\mathbf{S}_{\varepsilon, \mu}$, obtida de $\mathbf{S}$, através do $\varepsilon$-engrossamento e $\mu$-regularização. Consideramos que o contato de uma linha de curvatura principal com o bordo B é cúbico. Vimos que de tais pontos de contato cúbico emergem, sobre a superfície regularizada $\mathbf{S}_{\varepsilon, \mu}$, pontos umbílicos não Darbouxianos dos tipos $D_{12}$ e $D_{23}$, além dos pontos umbílicos Darbouxianos dos tipos $D_{1}, D_{2}$ e $D_{3}$. Veja teorema 4.3.4. 


\section{Capítulo 1}

\section{Levantamento de Lie-Cartan, Pontos Umbílicos e suas Bifurcações}

Este capítulo é introdutório ao estudo da configuração principal de superfícies em $\mathbb{R}^{3}$. Revisaremos alguns conceitos e resultados necessários para o bom desenvolvimento deste trabalho. Na seção 1.1 determinaremos a equação diferencial das linhas de curvatura principal para uma superfície imersa em $\mathbb{R}^{3}$ (equação 1.4 ). Na seção 1.2 utilizaremos o levantamento de Lie-Cartan (definição 1.2.1) para reduzir o estudo das linhas de curvatura principal, em uma vizinhança dos pontos umbílicos, ao estudo de curvas integrais de campos de linhas, em uma vizinhança dos seus equilíbrios. Este estudo foi feito por Bruce e Fidal em [1]. Veja também [9]. Na seção 1.3 apresentaremos dois padrões genéricos de bifurcações de pontos umbílicos, também conhecidos como pontos umbílicos de codimensão 1: o ponto umbílico do tipo $D_{12}$ (definição 1.3.1) e o ponto umbílico do tipo $D_{23}$ (definição 1.3.4.

\subsection{Equações Diferenciais das Linhas de Curvatura Principal}

Seja $\mathbf{S}$ uma superfície em $\mathbb{R}^{3}$, regular de classe $C^{r}(r \geq 5)$, compacta, conexa e orientada. Uma imersão $\alpha: \mathbf{S} \longrightarrow \mathbb{R}^{3}$ é uma aplicação tal que $D \alpha(\mathfrak{p}): T_{\mathfrak{p}} \mathbf{S} \longrightarrow \mathbb{R}^{3}$ é injetora, para cada $\mathfrak{p} \in \mathbf{S}$.

O espaço $\mathbb{R}^{3}$ está orientado e munido do produto interno Euclideano $\langle$,$\rangle .$

A métrica induzida sobre $T_{\mathfrak{p}} \mathbf{S}$ é definida por

$$
\langle u, v\rangle_{\mathfrak{p}}:=\langle D \alpha(\mathfrak{p}) u, D \alpha(\mathfrak{p}) v\rangle, \quad \text { onde } u, v \in T_{\mathfrak{p}} \mathbf{S}
$$

Denotemos por $\mathcal{N}_{\alpha}$ a aplicação normal unitária a $\alpha$ que define a orientação positiva da superfície $\mathbf{S}$. Isto significa que se $(u, v):(\mathbf{S}, \mathfrak{p}) \rightarrow\left(\mathbb{R}^{2}, 0\right)$ é uma carta positiva em torno 
de $\mathfrak{p}$, então $\left\{\alpha_{u}, \alpha_{v}, \mathcal{N}_{\alpha}\right\}$ é um referencial positivo em $\mathbb{R}^{3}$. Assim, a aplicação normal $\mathcal{N}_{\alpha}$ se exprime por $\mathcal{N}_{\alpha}=\left(\alpha_{u} \wedge \alpha_{v}\right) /\left|\alpha_{u} \wedge \alpha_{v}\right|$, onde $\wedge$ denota o produto vetorial em $\mathbb{R}^{3}$ e ||$=\langle,\rangle^{1 / 2}$ a norma Euclideana em $\mathbb{R}^{3}$.

A carta $(u, v)$ induz a base $\{d u, d v\}$ no espaço das formas lineares, sendo $\{d u, d v\}$ a base dual da base $\left\{\alpha_{u}, \alpha_{v}\right\}$ de $T_{\mathfrak{p}} \mathbf{S}$.

As formas fundamentais de $\mathbf{S}$, denotadas por $I_{\alpha}, I I_{\alpha}: T_{\mathfrak{p}} \mathbf{S} \longrightarrow \mathbb{R}$, na carta $(u, v)$ são dadas por

$$
\begin{aligned}
& I_{\alpha}(w)=E(\mathfrak{p}) d u^{2}+2 F(\mathfrak{p}) d u d v+G(\mathfrak{p}) d v^{2}=w P w^{T}, \\
& I I_{\alpha}(w)=e(\mathfrak{p}) d u^{2}+2 f(\mathfrak{p}) d u d v+g(\mathfrak{p}) d v^{2}=w Q w^{T},
\end{aligned}
$$

onde $E=\left\langle\alpha_{u}, \alpha_{u}\right\rangle, F=\left\langle\alpha_{u}, \alpha_{v}\right\rangle, G=\left\langle\alpha_{v}, \alpha_{v}\right\rangle, e=\left\langle\alpha_{u u}, \mathcal{N}_{\alpha}\right\rangle, f=\left\langle\alpha_{u v}, \mathcal{N}_{\alpha}\right\rangle$, $g=\left\langle\alpha_{v v}, \mathcal{N}_{\alpha}\right\rangle, w=(d u, d v), P=\left[\begin{array}{cc}E & F \\ F & G\end{array}\right]$ e $Q=\left[\begin{array}{ll}e & f \\ f & g\end{array}\right]$.

A curvatura normal $k_{n}: T_{\mathfrak{p}} \mathbf{S} \backslash\{0\} \longrightarrow \mathbb{R}$ no ponto $\mathfrak{p}$ e na direção $w=(d u, d v)$ é definida por $k_{n}(\mathfrak{p}, w)=\frac{I I_{\alpha}(w)}{I_{\alpha}(w)}$. As direções onde $k_{n}(\mathfrak{p},$.$) assume valores críticos são$ chamadas direções principais. Os valores de $k_{n}$ nestas direções são chamados curvaturas principais, as quais denotaremos por $k_{1}(\mathfrak{p}) \leq k_{2}(\mathfrak{p})$. Quando $k_{1}(\mathfrak{p})=k_{2}(\mathfrak{p})$, dizemos que $\mathfrak{p}$ é um ponto umbílico . O conjunto $\mathcal{U}_{\alpha}=\left\{\mathfrak{p} \in \mathbf{S} ; k_{1}(\mathfrak{p})=k_{2}(\mathfrak{p})\right\}$ será chamado conjunto umbílico da superfície $\mathbf{S}$.

Fora dos pontos umbílicos, as direções principais determinam dois campos de direções tangentes à superfície, mutuamente ortogonais, denominados campos de direções principais. Os pontos umbílicos são considerados singularidades destes campos.

Restringindo $k_{n}$ à condição $I_{\alpha}=1$, observamos que as direções principais onde $k_{n}(\mathfrak{p},$. assume valores críticos são dadas pelo método dos multiplicadores de Lagrange (com multiplicador $k$ ) resolvendo a equação

$$
\nabla I I_{\alpha}=k \nabla I_{\alpha}
$$

Neste caso, $k$ é uma das curvaturas principais. Portanto, tais curvaturas são definidas por

$$
\operatorname{det}(Q-k P)=0
$$

Da equação (1.1) temos que as direções principais são as soluções do seguinte sistema de equações lineares:

$$
\left\{\begin{array}{l}
\left(e-k_{i} E\right) d u+\left(f-k_{i} F\right) d v=0 \\
\left(f-k_{i} F\right) d u+\left(g-k_{i} G\right) d v=0
\end{array},\right.
$$

onde $k_{1}$ e $k_{2}$ são as curvaturas principais. O sistema $(1.3)$ descreve os campos de direções 
principais. As curvas integrais destes campos de direções são chamadas linhas de curvatura principal da superfície S. As folheações principais definidas pelo conjunto das linhas de curvatura principal (mínimas e máximas) serão denotadas por $\mathcal{F}_{1, \alpha}$ e $\mathcal{F}_{2, \alpha}$. A terna $\left(\mathcal{U}_{\alpha}, \mathcal{F}_{1, \alpha}, \mathcal{F}_{2, \alpha}\right)$ é chamada configuração principal da superfície $\mathbf{S}$.

A curvatura normal $k_{n}(\mathfrak{p}, w)$ na direção $[d u: d v]$, também denotada por $\lambda=\frac{d v}{d u}$, pode ser reescrita como:

$$
k_{n}(\mathfrak{p}, \lambda)=\frac{e(\mathfrak{p}) d u^{2}+2 f(\mathfrak{p}) d u d v+g(\mathfrak{p}) d v^{2}}{E(\mathfrak{p}) d u^{2}+2 F(\mathfrak{p}) d u d v+G(\mathfrak{p}) d v^{2}}=\frac{e(\mathfrak{p})+2 f(\mathfrak{p}) \lambda+g(\mathfrak{p}) \lambda^{2}}{E(\mathfrak{p})+2 F(\mathfrak{p}) \lambda+G(\mathfrak{p}) \lambda^{2}}
$$

Os valores extremos de $k_{n}$ são caracterizados por $\frac{d k_{n}}{d \lambda}=0$. Logo, efetuando-se este cálculo obtemos, a seguir, a equação diferencial das linhas de curvatura da superfície $\mathbf{S}$ :

$$
(F g-G f) \lambda^{2}+(E g-G e) \lambda+(E f-F e)=0
$$

ou equivalentemente,

$$
(F g-G f) d v^{2}+(E g-G e) d u d v+(E f-F e) d u^{2}=0 .
$$

Sendo $L=(F g-G f), M=(E g-G e)$ e $N=(E f-F e)$, podemos concluir que os pontos umbílicos da superfície $\mathbf{S}$ ocorrem em $L=M=N=0$.

\subsection{O Campo de Lie-Cartan e os Pontos Umbílicos Darbouxianos}

Uma ferramenta importante que utilizaremos se insere no estudo das equações diferenciais implícitas. Trata-se do campo de linhas de Lie-Cartan, que é um levantamento da equação diferencial das linhas de curvatura principal de $\mathbf{S}$ a uma superfície no fibrado projetivo PS sobre $\mathbf{S}$.

Denotemos por $\mathcal{I}^{r, s}=\mathcal{I}^{r, s}\left(\mathbf{S}, \mathbb{R}^{3}\right), s \leq r$, o conjunto das imersões de $\mathbf{S}$ em $\mathbb{R}^{3}$ de classe $C^{r}$ munido com a topologia $C^{s}$.

Para cada $\alpha \in \mathcal{I}^{r}=\mathcal{I}^{r, r}\left(\mathbf{S}, \mathbb{R}^{3}\right)$, considere a seguinte equação diferencial implícita

$$
\mathbf{L S}(u, v ;[d u: d v])=L d v^{2}+M d u d v+N d u^{2}=0
$$

a qual define uma superfície $\mathbb{L}_{\alpha}:=\mathbf{L S}^{-1}\{0\}$ no fibrado projetivo PS sobre $\mathbf{S}, 11$ com projeção $\pi: \mathbf{P S} \longrightarrow \mathbf{S}$. Esta superfície será chamada superfície de Lie-Cartan associada

\footnotetext{
${ }^{1} \mathbf{P S}$ é definido pela seguinte relação de equivalência: para $s_{1}, s_{2} \in \mathbf{S}$ e $v_{1}, v_{2} \in \mathbf{T S}$ (fibrado tangente de $\mathbf{S}),\left(\mathfrak{p}_{1}, v_{1}\right)$ é equivalente a $\left(\mathfrak{p}_{2}, v_{2}\right)$, se $\mathfrak{p}_{1}=\mathfrak{p}_{2}$ e $v_{1}$ e $v_{2}$ são colineares, isto é $v_{1} \wedge v_{2}=0$.
} 
à imersão $\alpha$.

A superfície de Lie-Cartan que acabamos de definir é de classe $C^{r-2}$ e, apesar de ser regular em $\mathbf{S} \backslash \mathcal{U}_{\alpha}$, pode apresentar singularidades sobre $\mathcal{U}_{\alpha}$.

Em termos da carta $(u, v)$ com domínio $V \subset \mathbf{S}$, são definidas duas cartas $(u, v ; p=d v / d u)$ e $(u, v ; q=d u / d v)$ cujos domínios cobrem o conjunto aberto $\pi^{-1}(V)$. $\mathrm{Na}$ carta $(u, v ; p=d v / d u), \mathbb{L}_{\alpha}$ é dada por:

$$
\mathcal{L}(u, v ; p)=L(u, v) p^{2}+M(u, v) p+N(u, v)=0,
$$

e na carta $(u, v ; q=d u / d v)$, por:

$$
\mathcal{G}(u, v ; q)=L(u, v)+M(u, v) q+N(u, v) q^{2}=0 .
$$

Definição 1.2.1. Na carta $(u, v ; p), p=\frac{d v}{d u}$, o campo de linhas de Lie-Cartan (ou levantamento da equação diferencial das linhas de curvatura principal), de classe $C^{r-3}$, é definido por:

$$
X_{\alpha}:=\mathcal{L}_{p} \frac{\partial}{\partial u}+p \mathcal{L}_{p} \frac{\partial}{\partial v}-\left(\mathcal{L}_{u}+p \mathcal{L}_{v}\right) \frac{\partial}{\partial p} .
$$

Na carta $(u, v ; q), q=\frac{d u}{d v}$, o campo de linhas tem a seguinte expressão:

$$
Y_{\alpha}:=q \mathcal{G}_{q} \frac{\partial}{\partial u}+\mathcal{G}_{q} \frac{\partial}{\partial v}-\left(\mathcal{G}_{v}+q \mathcal{G}_{u}\right) \frac{\partial}{\partial q} .
$$

As projeções das curvas integrais de $X_{\alpha}$ por $\pi(u, v ; p)=(u, v)$ são as linhas de curvatura principal da superfície $\mathbf{S}$ e satisfazem $p=d v / d u$. Além disso, o campo $X_{\alpha}$ é tangente à superfície de Lie-Cartan, pois

$$
X_{\alpha} \mathcal{L}=\mathcal{L}_{u} \mathcal{L}_{p}+p \mathcal{L}_{v} \mathcal{L}_{p}-\mathcal{L}_{p} \mathcal{L}_{u}-p \mathcal{L}_{p} \mathcal{L}_{v}=0
$$

Definição 1.2.2. Um ponto $\mathfrak{p} \in \mathcal{U}_{\alpha}$ é Darbouxiano se satisfaz às condições $T$ ) e D) abaixo:

T): (Condição de Transversalidade) A superfície de Lie-Cartan, $\mathbb{L}_{\alpha}$, é regular também sobre $\pi^{-1}(\mathfrak{p})$.

D): (Condição do Discriminante) As singularidades do campo de Lie-Cartan sobre $\pi^{-1}(\mathfrak{p})$ são hiperbólicas e dadas por um dos seguintes casos:

$D_{1}$ : uma única sela, ou

$D_{2}$ : uma único nó entre duas selas, ou 
$D_{3}$ : três selas.

Para efeito de cálculos, será útil expressar as condições Darbouxianas em uma carta local apropriada: seja $\mathfrak{p} \in \mathcal{U}_{\alpha}$ e suponha $\alpha$ expressa na carta de Monge $(u, v):(\mathbf{S}, \mathfrak{p}) \rightarrow\left(\mathbb{R}^{2}, 0\right)$ por $\alpha(u, v)=(u, v, h(u, v))$, sendo

$$
\begin{aligned}
h(u, v) & =\frac{k}{2}\left(u^{2}+v^{2}\right)+\frac{a}{6} u^{3}+\frac{b}{2} u v^{2}+\frac{c}{6} v^{3}+\frac{A}{24} u^{4}+\frac{B}{6} u^{3} v+ \\
& +\frac{C}{4} u^{2} v^{2}+\frac{D}{6} u v^{3}+\frac{E}{24} v^{4}+O(5) .
\end{aligned}
$$

Nesta expressão o coeficiente de $u^{2} v$ pode ser feito nulo por meio de uma rotação no plano $(u, v)$. A equação diferencial das linhas de curvatura principal da superfície $\mathbf{S}$ próxima do ponto umbílico $\mathfrak{p}$ é dada por:

$$
-\left[b v+P_{1}\right] d v^{2}+\left[(b-a) u+c v+P_{2}\right] d u d v+\left[b v+P_{3}\right] d u^{2}=0
$$

onde $P_{i}(i=1,2,3)$ representam funções de ordem $O\left(u^{2}+v^{2}\right)$.

Observe que, na carta $(u, v ; p)$, as singularidades do campo de Lie-Cartan $X_{\alpha}$ (equação (1.8)) são dadas por $\left(0,0 ; p_{i}\right)$, sendo $p_{i}(i=0,1,2)$ raízes do polinômio

$$
p\left(b p^{2}-c p+a-2 b\right)=0 .
$$

Segue da condição de transversalidade $T$ que $b \neq 0$ (veja demonstração do teorema $1.2 .3 \mathrm{em}\left[7\right.$, 8] ), logo as singularidades de $X_{\alpha}$ sobre a superfície $\mathbb{L}_{\alpha}$ estão localizadas ao longo do eixo $p$, cujas coordenadas $p_{0}, p_{1}$ e $p_{2}$ são dadas por

$$
\begin{aligned}
& p_{0}=0, \quad p_{1}=\frac{c}{2 b}-\sqrt{\left(\frac{c}{2 b}\right)^{2}-\frac{a}{b}+2}, \\
& p_{2}=\frac{c}{2 b}+\sqrt{\left(\frac{c}{2 b}\right)^{2}-\frac{a}{b}+2}
\end{aligned}
$$

A superfície implícita $\mathcal{L}(u, v ; p)=L(u, v) p^{2}+M(u, v) p+N(u, v)=0$ é regular em uma vizinhança da linha projetiva se, e somente se, $b(b-a) \neq 0$. Próximo do ponto singular $p_{0}=(0,0,0)$ de $X_{\alpha}$ segue que $\mathcal{L}_{v}\left(p_{0}\right)=b \neq 0$ e, portanto, pelo Teorema da Função Implícita, exite uma função $v=v(u, p)$ tal que $\mathcal{L}(u, v(u, p) ; p)=0$. Assim, temos a seguinte expansão de Taylor para a função $v$ :

$$
v(u, p)=-\frac{B}{2 b} u^{2}+\frac{a-b}{b} u p+O(3) .
$$

A expressão do campo de Lie-Cartan $X_{\alpha}$ na carta $(u, p)$ é dada por: 


$$
\begin{aligned}
\dot{u} & =\mathcal{L}_{p}(u, v(u, p) ; p)=(b-a) u+\frac{1}{2} \frac{\left[b\left(C-A+2 k^{3}\right)-c B\right]}{b} u^{2}+\frac{c(a-b)}{b} u p+O(3) \\
\dot{p} & =-\left(\mathcal{L}_{u}+p \mathcal{L}_{v}\right)(u, v(u, p) ; p)=-B u+(a-2 b) p-c p^{2}+ \\
& +\frac{1}{2} \frac{\left[B\left(C-k^{3}\right)-a_{41} b\right]}{b} u^{2}+\frac{\left[b\left(A-C-2 k^{3}\right)+a\left(k^{3}-C\right)\right]}{b} u p+O(3)
\end{aligned}
$$

onde $a_{41}=\left.\frac{\partial^{5} h}{\partial u^{4} \partial v}\right|_{u=v=0}$. Entretanto, $a_{41}$ não terá influência na análise qualitativa que segue.

O teorema 1.2.3, abaixo, relaciona as condições de Darboux $T$ ) e $D$ ) da definição 1.2 .2 com os coeficientes da parametrização de $\mathbf{S}$ na carta de Monge.

Teorema 1.2.3 (Gutierrez e Sotomayor, [7, 8]). Seja $\mathfrak{p}$ um ponto umbílico de uma imersão $\alpha$ de classe $C^{r}, r \geq 4$, expressa na carta de Monge $(u, v):(\mathbf{S}, \mathfrak{p}) \rightarrow\left(\mathbb{R}^{2}, 0\right)$ por $\alpha(u, v)=(u, v, h(u, v))$, sendo

$$
h(u, v)=\frac{k}{2}\left(u^{2}+v^{2}\right)+\frac{a}{6} u^{3}+\frac{b}{2} u v^{2}+\frac{c}{6} v^{3}+O(4) .
$$

Suponha que as seguintes condições são satisfeitas:

T) $b(b-a) \neq 0$;

D) $\left(\frac{c}{2 b}\right)^{2}-\frac{a}{b}+2<0$

$\left.D_{2}\right)\left(\frac{c}{2 b}\right)^{2}+2>\frac{a}{b}>1, \quad a \neq 2 b$

$\left.D_{3}\right) \frac{a}{b}<1$

Então, cada folheação principal tem, em uma vizinhança da origem, um setor hiperbólico no caso $D_{1}$, um setor parabólico e um hiperbólico no caso $D_{2}$ e três setores hiperbólicos no caso $D_{3}$. Os umbílicos são chamados Darbouxianos dos tipos $D_{1}, D_{2}$ e $D_{3}$. Veja figura 1.1 .

O subscrito $(i=1,2,3)$ de $D_{i}$ denota o número de separatrizes umbílicas de $\mathfrak{p}$, que são linhas de curvatura principal tendendo a $\mathfrak{p}$ e que separam regiões de diferentes padrões de aproximação a $\mathfrak{p}$.

Outras propriedades gerais das linhas de curvatura e dos pontos umbílicos podem ser encontradas em [6, 13]. 


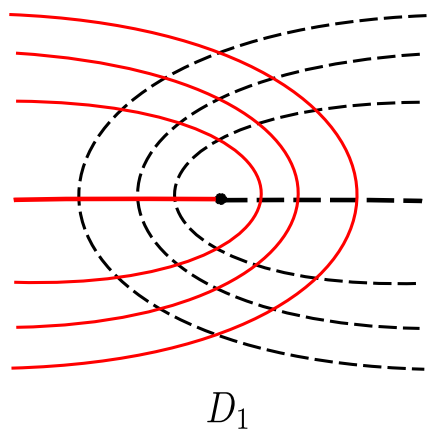

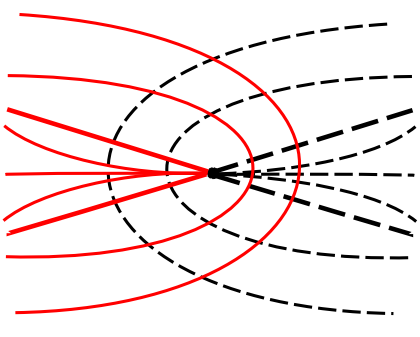

$D_{2}$

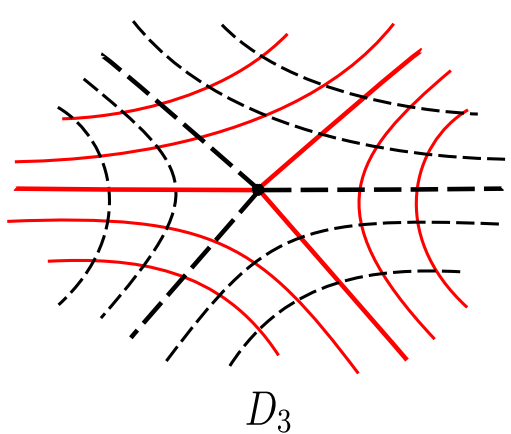

Figura 1.1: Linhas de curvaturas principais próximas aos pontos umbilicos $D_{i}$ e suas separatrizes umbilicas, no bordo dos setores.

\subsection{Pontos Umbílicos de Codimensão 1}

Dois padrões genéricos de bifurcações de pontos umbílicos surgem em codimensão 1. O primeiro deles, chamado $D_{12}$, ocorre quando a condição do discriminante $D$ é desrespeitada, enquanto a condição de transversalidade $T$ é preservada. O segundo padrão, chamado de $D_{23}$, ocorre quando a condição $T$ é desrespeitada e a condição $D$ é preservada. A expressão codimensão 1 significa que as mudanças qualitativas na configuração principal das linhas de curvatura próximas dos pontos umbílicos estão bem descritas por uma família a 1 parâmetro $\alpha_{\lambda}$ de imersões de uma superfície $\mathbf{S}$ em $\mathbb{R}^{3}$, onde $\lambda \in \mathbb{R}$. $\mathrm{O}$ espaço destas famílias está munido com a topologia de Whitney.

\subsubsection{O Padrão de Bifurcação do Ponto Umbílico $D_{12}$}

Vamos estudar as mudanças qualitativas da configuração principal na vizinhança de um ponto umbílico não Darbouxiano que ocorrem quando a condição de transversalidade $T, b(b-a) \neq 0$, é preservada e somente a condição $D$ é desrespeitada da maneira mais simples possível.

Definição 1.3.1. O ponto $\mathfrak{p} \in \mathcal{U}_{\alpha}$ é chamado ponto umbílico do tipo $D_{12}$ se satisfaz às condições $T$ ) e $D_{12}$ ) abaixo:

T) A superfície de Lie-Cartan, $\mathbb{L}_{\alpha}$, é regular também sobre $\pi^{-1}(\mathfrak{p})$.

$\left.D_{12}\right)$ As singularidades do campo de Lie-Cartan são uma sela hiperbólica e uma sela-nó cuja variedade central está situada ao longo da linha projetiva sobre $\mathfrak{p}$.

Considerando a mesma notação da seção 1.2, seja p um ponto umbílico da imersão $\alpha$ expressa na carta de Monge $(u, v):(\mathbf{S}, \mathfrak{p}) \rightarrow\left(\mathbb{R}^{2}, 0\right)$ por $\alpha(u, v)=(u, v, h(u, v))$, sendo

$$
\begin{aligned}
h(u, v) & =\frac{k}{2}\left(u^{2}+v^{2}\right)+\frac{a}{6} u^{3}+\frac{b}{2} u v^{2}+\frac{b^{\prime}}{2} u^{2} v+\frac{c}{6} v^{3}+\frac{A}{24} u^{4}+\frac{B}{6} u^{3} v+ \\
& +\frac{C}{4} u^{2} v^{2}+\frac{D}{6} u v^{3}+\frac{E}{24} v^{4}+O(5),
\end{aligned}
$$


onde assumimos, como antes, que o coeficiente $b^{\prime}$ pode ser considerado nulo.

A caracterização do ponto umbílico $D_{12}$, da definição 1.3.1, é dada em termos da carta de Monge como segue:

T) $b(b-a)>0$ e, ou

(1) $\frac{a}{b}=\left(\frac{c}{2 b}\right)^{2}+2$, ou

(2) $\frac{a}{b}=2$.

O ponto umbílico $D_{12}$ possui duas separatrizes umbílicas: a separatriz isolada e a separatriz não-isolada. A separatriz isolada caracteriza-se pelo fato de não possuir tangência com qualquer outra linha de curvatura que também se aproxima do ponto umbílico. A separatriz não-isolada possui tangência com as linhas de curvatura principal que se aproximam do ponto umbílico, exceto com a separatriz isolada. Veja figura 1.2, na qual as separatrizes umbílicas estão hachuradas.

Os coeficientes $L=F g-G f, M=E g-G e$ e $N=E f-F e$ da equação diferencial das linhas de curvatura principal $L(u, v) d v^{2}+M(u, v) d u d v+N(u, v) d u^{2}=0$ são dados por:

$$
\begin{aligned}
L(u, v) & =-b v-\frac{B}{2} u^{2}-\left(C-k^{3}\right) u v-\frac{D}{2} v^{2}+O(3) \\
M(u, v) & =(b-a) u+c v+\left[\frac{C-A}{2}+k^{3}\right] u^{2}+(D-B) u v+ \\
& +\left[\frac{E-C}{2}-k^{3}\right] v^{2}+O(3) \\
N(u, v) & =b v+\frac{B}{2} u^{2}+\left(C-k^{3}\right) u v+\frac{D}{2} v^{2}+O(3) .
\end{aligned}
$$

A condição $D_{12}$ é equivalente à existência de uma raiz dupla e não nula do polinômio $b p^{2}-c p+a-2 b=0$ (sendo $p=d v / d u$ ), que corresponde a $b \neq 0$ e $p_{1}=p_{2} \neq p_{0}$. Portanto, assumindo que $b(b-a) \neq 0$, as curvas $L(u, v)=0$ e $M(u, v)=0$ interceptam-se transversalmente em $(0,0)$ se, e somente se, $b \neq a$.

Foi demonstrado em [7] por Gutierrez e Sotomayor que a condição $D_{1}$ é satisfeita se, se somente se, as raízes do polinômio $b p^{2}-c p+a-2 b=0$ são não nulas e puramente imaginárias. Também que a condição $D_{2}$ é satisfeita se, e somente se, as raízes $p_{1}$ e $p_{2}$ de $b p^{2}-c p+a-2 b=0$ são distintas, não nulas e satisfazem $p_{1} \cdot p_{2}>-1$.

Proposição 1.3.2 ([6], proposição 4.2.1). Suponha que $\alpha \in \mathcal{I}^{r}, r \geq 5$, satisfaz a condição $D_{12}$ em um ponto umbílico $\mathfrak{p}$. Então, a configuração principal local de $\alpha$ em torno de $\mathfrak{p} e ́$ como na figura 1.2, centro. 
Demonstração. Considere o campo de Lie-Cartan na carta $p=d v / d u$ como na expressão (1.8). Se $a=2 b \neq 0$ e $c \neq 0$, temos que $p_{0}=(0,0,0)$ é um ponto singular isolado tipo sela-nó com sua separatriz central contida na linha projetiva, que neste caso é o eixo $p$. De fato, os autovalores de $D X_{\alpha}(0)$ são $\lambda_{1}=-b \neq 0$ e $\lambda_{2}=0$ e o eixo $p$ é invariante, sendo $X_{\alpha}$ dado por $\dot{p}=-c p^{2}+O(2)$, segundo a equação 1.10 .

O outro ponto singular de $X_{\alpha}$ é dado por $p_{1}=(0,0, c / b)$. Assim,

$$
D X_{\alpha}\left(0,0 ; p_{1}\right)=\left[\begin{array}{ccc}
-b & -c & 0 \\
-c & -\frac{c^{2}}{b} & 0 \\
B_{1} & B_{2} & \frac{c^{2}}{b}
\end{array}\right]
$$

onde

$$
\begin{aligned}
& B_{1}=\frac{b^{2} c\left(A-k^{3}-2 C\right)+b c^{2}(2 B-D)+c^{3}\left(C-k^{3}\right)-b^{3} D}{b^{3}}, \\
& B_{2}=\frac{b^{2} c(B-2 D)+b c^{2}\left(2 C+k^{3}-E\right)+b^{3}\left(k^{3}-C\right)+D c^{3}}{b^{3}} .
\end{aligned}
$$

Os autovalores não nulos de $D X_{\alpha}\left(0,0, p_{1}\right)$ são

$$
\lambda_{1}=\frac{c^{2}}{b} \quad \text { e } \quad \lambda_{2}=-\frac{c^{2}+b^{2}}{b} .
$$

Portanto, $p_{1}$ é um ponto de sela hiperbólica de $X_{\alpha}$ com autovalores $\lambda_{1}$ e $\lambda_{2}$.

Uma análise similar pode ser feita quando $\left(\frac{c}{2 b}\right)^{2}-\frac{a}{b}+2=0$.

Isto completa a demonstração.

Proposição 1.3.3 ([6], proposição 4.2.2). Suponha que $\alpha \in \mathcal{I}^{r}, r \geq 5$, satisfaz a condição $D_{12}$ em um ponto umbílico $\mathfrak{p}$. Então, existe uma vizinhança $V$ de $\mathfrak{p}$, uma vizinhança $\mathcal{V}$ de $\alpha$ e uma função $\mathcal{B}: \mathcal{V} \longrightarrow \mathbb{R}$ de classe $C^{r-3}$ tal que cada $\beta \in \mathcal{V}$ tem um único ponto umbílico $\mathfrak{p}_{\beta}$ em $V$, sendo:

(i) $d \mathcal{B}(\alpha) \neq 0$,

(ii) $\mathcal{B}(\beta)>0$ se, e somente se, $\mathfrak{p}_{\beta}$ é Darbouxiano do tipo $D_{1}$,

(iii) $\mathcal{B}(\beta)<0$ se, e somente se, $\mathfrak{p}_{\beta}$ é Darbouxiano do tipo $D_{2}$,

(iv) $\mathcal{B}(\beta)=0$ se, e somente se, $\mathfrak{p}_{\beta}$ é do tipo $D_{12}$.

A configuração principal de $\beta$ em torno de $\mathfrak{p}$ é como na figura 1.2 , direita, esquerda e centro, respectivamente. 


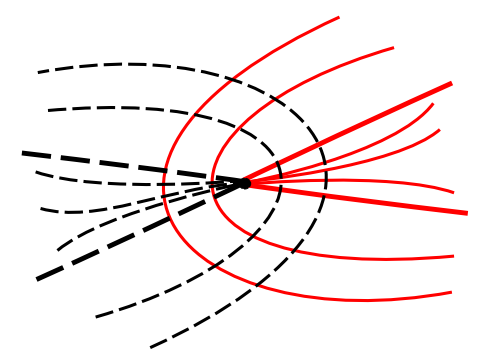

$D_{2}$

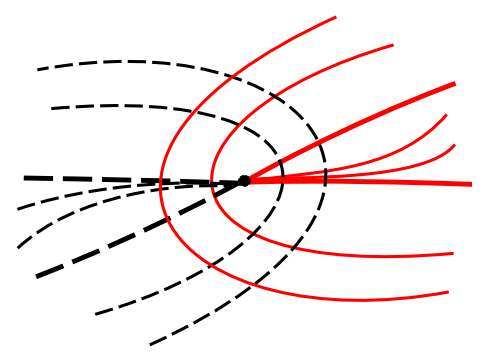

$D_{12}$

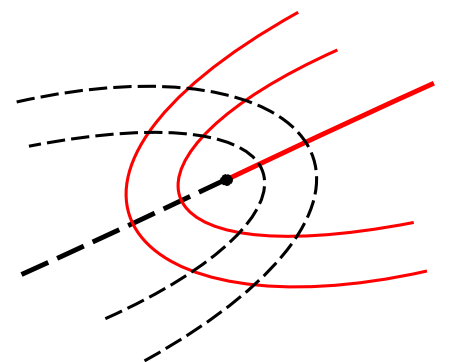

$D_{1}$

Figura 1.2: Ponto umbílico $D_{12}$ e sua bifurcação.

\subsubsection{O Padrão de Bifurcação do Ponto Umbílico $D_{23}$}

Iremos estudar as mudanças qualitativas da configuração principal na vizinhança de um ponto umbílico não Darbouxiano, chamado $D_{23}$, que ocorrem quando a condição de transversalidade $T$ é desrespeitada.

Definição 1.3.4. O ponto $\mathfrak{p} \in \mathcal{U}_{\alpha}$ é um ponto umbílico do tipo $D_{23}$ se a regularidade da supefície $\mathbb{L}_{\alpha}$ falha em dois pontos sobre o ponto umbílico, onde a superfície $\mathbb{L}_{\alpha}$ é não degenerada do tipo Morse.

Proposição 1.3.5 ([6], proposição 4.2.3). Suponha que $\alpha \in \mathcal{I}^{r}, r \geq 5, \mathfrak{p} \in \mathcal{U}_{\alpha} e$ considere a carta de Monge dada pela expressão (1.11) com $b^{\prime}=0, b=a \neq 0$ e $\chi=b\left(C-A+2 k^{3}\right)-c B \neq 0$. Então $\mathfrak{p}$ é do tipo $D_{23}$ e a configuração principal local de $\alpha$ em torno de $\mathfrak{p}$ é como na figura 1.3 , centro.

Demonstração. Pode ser encontrada em [6], p. 94 e também em [9], p. 333.

Observe que o contato das curvas $M=0$ e $N=0$ no ponto umbílico é quadrático. De fato, da equação (1.12) segue, pelo Teorema da Função Implícita, que $M(u, v(u))=0$ para $v=\frac{\chi+c B}{2 b} u^{2}+O(2)$ de classe $C^{r-2}$. Portanto, $n(u)=N(u, v(u))$ é de classe $C^{r-2}$ e $n(u)=\frac{\chi+c B}{2} u^{2}+O(2)$.

Observação 1.3.6. As seguintes três condições são equivalentes:

(i) a existência de exatamente dois pontos críticos de $\mathbb{L}_{\alpha}$ não degenerados do tipo Morse ao longo do eixo projetivo;

(ii) a existência de um ponto regular de $\mathbb{L}_{\alpha}$ sobre o eixo projetivo, o qual é um equilíbrio do tipo sela-nó do campo de Lie-Cartan com variedade central transversal ao eixo projetivo;

(iii) o contato quadrático das curvas $M=0$ e $N=0$ no ponto umbílico. 
Note que as folheações principais em torno do ponto umbílico $D_{23}$ são topologicamente distintas, o que difere dos outros pontos umbílicos discutidos até o momento. Uma delas, localizada na folha de paralelos possui duas separatrizes umbílicas e dois setores hiperbólicos. A outra, localizada na folha de sela-nó possui três separatrizes umbílicas, um setor parabólico e dois setores hiperbólicos.

A bifurcação $D_{23}$ é, portanto, uma transição entre a situação em que temos dois pontos umbílicos Darbouxianos, um do tipo $D_{2}$ e outro do tipo $D_{3}$ para a situação em que não há pontos umbílicos. Veja figura 1.3 na qual as folheações máxima e mínima foram desenhadas separadamente.

Proposição 1.3.7 ([6], proposição 4.2.4). Suponha que $\alpha \in \mathcal{I}^{r}, r \geq 5$, satisfaz a condição $D_{23}$ em um ponto umbílico $\mathfrak{p}$. Então, existe uma vizinhança $V$ de $\mathfrak{p}$, uma vizinhança $\mathcal{V}$ de $\alpha$ e uma função $\mathcal{B}: \mathcal{V} \longrightarrow \mathbb{R}$ de classe $C^{r-3}$ tal que:

(i) $d \mathcal{B}(\alpha) \neq 0$,

(ii) $\mathcal{B}(\beta)>0$ se, e somente se, $\beta$ não possui pontos umbílicos em $V$,

(iii) $\mathcal{B}(\beta)<0$ se, e somente se, $\beta$ possui dois pontos umbilicos Darbouxianos de tipos $D_{2}$ e $D_{3}$,

(iv) $\mathcal{B}(\beta)=0$ se, e somente se, $\beta$ possui um único ponto umbílico em $V$, o qual é do tipo $D_{23}$.

A configuração principal de $\beta$ em torno de $\mathfrak{p}$ é como na figura 1.3, direita, esquerda e centro, respectivamente.
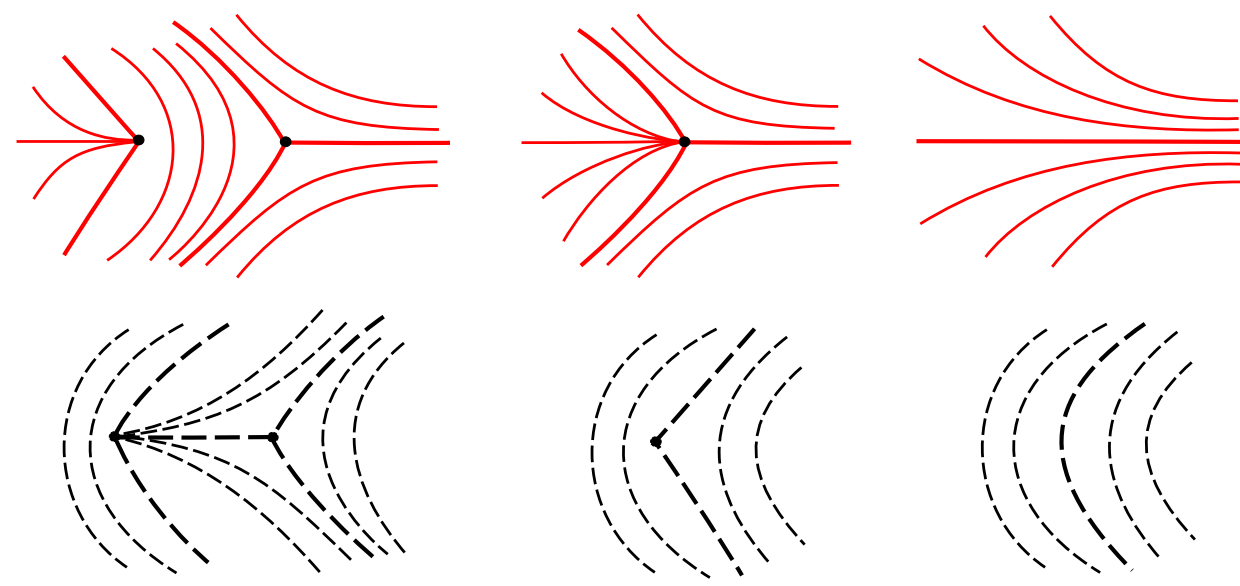

$$
D_{2}-D_{3}
$$

$D_{23}$

Figura 1.3: Bifurcação do ponto umbilico $D_{23}$. Os pontos umbilicos $D_{2}$ e $D_{3}$ colidem formando o ponto $D_{23}$, e depois não há pontos umbílicos. Fixada uma orientação, as linhas tracejadas (respectivamente contínuas) ilustram as linhas de curvatura principal da folheação principal minima, $\mathcal{F}_{1, \alpha}$ (respectivamente máxima, $\mathcal{F}_{2, \alpha}$ ). 


\section{Capítulo 2}

\section{Configuração Principal sobre Superfícies Suaves por Partes e Curva de Descontinuidade Plana}

Sejam $\mathbf{S}^{+}$e $\mathbf{S}^{-}$duas superfícies em $\mathbb{R}^{3}$, ambas de classe $C^{\infty}$ tendo como bordo comum uma curva plana $\boldsymbol{\delta}$. Considere $\mathbf{S}=\mathbf{S}^{+} \cup \boldsymbol{\delta} \cup \mathbf{S}^{-}$a superfície obtida pela justaposição de $\mathbf{S}^{+}$e $\mathbf{S}^{-}$ao longo do bordo comum $\boldsymbol{\delta}$. Neste capítulo serão estudadas as linhas de curvatura principal da superfície seccionalmente suave (ou suave por partes), $\mathbf{S}=\mathbf{S}^{+} \cup$ $\boldsymbol{\delta} \cup \mathbf{S}^{-} \subset \mathbb{R}^{3}$, e da superfície suave, $\mathbf{S}_{\varepsilon}$, obtida através da $\varepsilon$-regularização de $\mathbf{S}$ ao longo da curva de descontinuidade $\boldsymbol{\delta}, \varepsilon>0$ suficientemente pequeno (veja definição 2.2.2. . Suporemos inicialmente que $\boldsymbol{\delta}$ é uma curva plana (contida no plano $v=0$ ) e que $\mathbf{S}^{ \pm}$estão parametrizadas, sem perda de generalidade, por gráficos em uma vizinhança da origem.

Analisaremos três casos relativos à configuração principal da superfície suave por partes S: quando a origem é um ponto de tangência quadrática, um ponto de cruzamento transversal (proposições 2.1.1 e 2.1.3) ou um ponto de dobra (definição 2.1.10). Na seção 2.1 apresentaremos e definiremos com precisão cada um desses casos antes de aplicarmos a regularização. Na seção 2.2 definiremos a $\varepsilon$-regularização e estudaremos seus efeitos nos três casos citados acima. Veremos que, sob certas condições (estabelecidas nos teoremas 2.2.6, 2.2.10 2.2.11), de tais pontos do bordo comum emergem, sobre $\mathbf{S}_{\varepsilon}$, pontos umbílicos Darbouxianos dos tipos $D_{1}$ e $D_{3}$, para $\varepsilon>0$ suficientemente pequeno.

\subsection{Configuração das Linhas de Curvatura Principal de uma Superfície Suave por Partes}

Nesta seção será apresentada a formulação do primeiro problema abordado neste trabalho. Trata-se do caso em que o bordo $\boldsymbol{\delta}$, comum às superfícies $\mathbf{S}^{+}$e $\mathbf{S}^{-}$, é uma curva plana em $\mathbb{R}^{3}$. As proposições 2.1.1 e 2.1.3 estabelecem as condições para que a origem seja 
um ponto de tangência quadrática ou um ponto de cruzamento transversal tanto para a superfície $\mathbf{S}^{+}$quanto para a superfície $\mathbf{S}^{-}$.

\subsection{1 $1^{\circ}$ Caso: Singularidade Quadrática}

Sejam $\mathbf{S}^{+}$e $\mathbf{S}^{-}$duas superfícies em $\mathbb{R}^{3}$, ambas de classe $C^{\infty}$ tendo como bordo uma curva plana que definiremos a seguir. Sem perda de generalidade, vamos tomar parametrizações de $\mathbf{S}^{+}$e $\mathbf{S}^{-}$como gráficos em uma vizinhança da origem. Mais precisamente, na carta $(u, v)$ definida em uma vizinhança da origem, consideremos as parametrizações $X^{+}(u, v)=\left(u, v, h^{+}(u, v)\right)$ e $X^{-}(u, v)=\left(u, v, h^{-}(u, v)\right)$ das superfícies $\mathbf{S}^{+}$e $\mathbf{S}^{-}$, respectivamente, onde $h^{+}, h^{-} \in C^{\infty}$ são dadas por

$$
\begin{aligned}
h^{+}(u, v) & =\frac{1}{2} k_{1}^{+} u^{2}+k_{3}^{+} u v+\frac{1}{2} k_{2}^{+} v^{2}+\frac{1}{6} a u^{3}+\frac{1}{2} b u v^{2}+\frac{1}{2} c u^{2} v+\frac{1}{6} d v^{3}+ \\
& +\frac{1}{24} A u^{4}+\frac{1}{6} B u^{3} v+\frac{1}{4} C u^{2} v^{2}+\frac{1}{6} D u v^{3}+\frac{1}{24} E v^{4}+O(5)
\end{aligned}
$$

definida para $v>0$, e

$$
\begin{aligned}
h^{-}(u, v) & =\frac{1}{2} k_{1}^{-} u^{2}+k_{3}^{-} u v+\frac{1}{2} k_{2}^{-} v^{2}+\frac{1}{6} \alpha u^{3}+\frac{1}{2} \beta u v^{2}+\frac{1}{2} \gamma u^{2} v+\frac{1}{6} \rho v^{3}+ \\
& +\frac{1}{24} A_{1} u^{4}+\frac{1}{6} B_{1} u^{3} v+\frac{1}{4} C_{1} u^{2} v^{2}+\frac{1}{6} D_{1} u v^{3}+\frac{1}{24} E_{1} v^{4}+O(5),
\end{aligned}
$$

definida para $v<0.17$

Vamos supor que $h^{+}(u, 0)=h^{-}(u, 0)$ para $v=0$. Portanto, comparando os coeficientes de (2.1) e 2.2), para que $h^{+}(u, 0)=h^{-}(u, 0)$, devemos ter $k_{1}^{+}=k_{1}^{-}, a=\alpha$ e $A=A_{1}$. Assim, considerando $k_{1}=k_{1}^{+}=k_{1}^{-}$, podemos reescrever 2.1) e 2.2), respectivamente, como

$$
\begin{aligned}
h^{+}(u, v) & =\frac{1}{2} k_{1} u^{2}+k_{3}^{+} u v+\frac{1}{2} k_{2}^{+} v^{2}+\frac{1}{6} a u^{3}+\frac{1}{2} b u v^{2}+\frac{1}{2} c u^{2} v+\frac{1}{6} d v^{3}+ \\
& +\frac{1}{24} A u^{4}+\frac{1}{6} B u^{3} v+\frac{1}{4} C u^{2} v^{2}+\frac{1}{6} D u v^{3}+\frac{1}{24} E v^{4}+O(5),
\end{aligned}
$$

\footnotetext{
${ }^{1}$ Os sobrescritos "+" e "-" das superfícies $\mathbf{S}^{+}$e $\mathbf{S}^{-}$, bem como para suas respectivas parametrizações $X^{+}=\left(u, v, h^{+}\right)$e $X^{-}=\left(u, v, h^{-}\right)$, referem-se à parte superior (quando $v>0$ ) e à parte inferior (quando $v<0$ ) do plano $u v$.
} 
definida para $v \geq 0$, e

$$
\begin{aligned}
h^{-}(u, v) & =\frac{1}{2} k_{1} u^{2}+k_{3}^{-} u v+\frac{1}{2} k_{2}^{-} v^{2}+\frac{1}{6} a u^{3}+\frac{1}{2} \beta u v^{2}+\frac{1}{2} \gamma u^{2} v+\frac{1}{6} \rho v^{3}+ \\
& +\frac{1}{24} A u^{4}+\frac{1}{6} B_{1} u^{3} v+\frac{1}{4} C_{1} u^{2} v^{2}+\frac{1}{6} D_{1} u v^{3}+\frac{1}{24} E_{1} v^{4}+O(5),
\end{aligned}
$$

definida para $v \leq 0$.

A curva parametrizada por $\delta(u)=\left(u, 0, h^{+}(u, 0)\right)=\left(u, 0, h^{-}(u, 0)\right)$, contida no plano $v=0$, é o bordo comum das superfícies $\mathbf{S}^{+}$e $\mathbf{S}^{-}$, onde $\boldsymbol{\delta}=\{\delta(u), u \in \mathbb{R}\}$.

A seguir calcularemos os coeficientes das formas fundamentais das superfícies $\mathbf{S}^{+}$e $\mathbf{S}^{-}$ na carta $(u, v)$, os quais serão úteis, posteriormente, na descrição da equação diferencial das linhas de curvatura destas superfícies.

Para a superfície $\mathbf{S}^{+}$, considerando a parametrização $X^{+}(u, v)=\left(u, v, h^{+}(u, v)\right)$ e sendo $h^{+}$como em 2.3 temos:

$$
\begin{aligned}
\frac{\partial h^{+}}{\partial u} & =k_{1} u+k_{3}^{+} v+\frac{1}{2} a u^{2}+c u v+\frac{1}{2} b v^{2}+\frac{1}{6} A u^{3}+\frac{1}{2} B u^{2} v+\frac{1}{2} C u v^{2}+\frac{1}{6} D v^{3}+O(4), \\
\frac{\partial h^{+}}{\partial v} & =k_{3}^{+} u+k_{2}^{+} v+\frac{1}{2} c u^{2}+b u v+\frac{1}{2} d v^{2}+\frac{1}{6} B u^{3}+\frac{1}{2} C u^{2} v+\frac{1}{2} D u v^{2}+\frac{1}{6} E v^{3}+O(4), \\
\frac{\partial^{2} h^{+}}{\partial u^{2}} & =k_{1}+a u+c v+\frac{1}{2} A u^{2}+B u v+\frac{1}{2} C v^{2}+O(3), \\
\frac{\partial^{2} h^{+}}{\partial u \partial v} & =k_{3}^{+}+c u+b v+\frac{1}{2} B u^{2}+C u v+\frac{1}{2} D v^{2}+O(3), \\
\frac{\partial^{2} h^{+}}{\partial v^{2}} & =k_{2}^{+}+b u+d v+\frac{1}{2} C u^{2}+D u v+\frac{1}{2} E v^{2}+O(3) .
\end{aligned}
$$

O vetor normal (não unitário) a $\mathbf{S}^{+}$é dado por:

$$
\mathcal{N}^{+}(u, v)=\left(\frac{\partial X^{+}}{\partial u} \wedge \frac{\partial X^{+}}{\partial v}\right)(u, v)=\left(-\frac{\partial h^{+}}{\partial u},-\frac{\partial h^{+}}{\partial v}, 1\right)
$$

Os coeficientes da Primeira Forma Fundamental de $X^{+}$expressos na carta $(u, v)$ são:

$$
\begin{aligned}
E & =\left\langle\frac{\partial X^{+}}{\partial u}, \frac{\partial X^{+}}{\partial u}\right\rangle=\left\langle\left(1,0, \frac{\partial h^{+}}{\partial u}\right),\left(1,0, \frac{\partial h^{+}}{\partial u}\right)\right\rangle=1+k_{1}^{2} u^{2}+2 k_{1} k_{3}^{+} u v+ \\
& +\left(k_{3}^{+}\right)^{2} v^{2}+k_{1} a u^{3}+\left(2 k_{3}^{+} c+k_{1} b\right) u v^{2}+\left(2 k_{1} c+k_{3}^{+} a\right) u^{2} v+k_{3}^{+} b v^{3}+O(4),
\end{aligned}
$$




$$
\begin{aligned}
F & =\left\langle\frac{\partial X^{+}}{\partial u}, \frac{\partial X^{+}}{\partial v}\right\rangle=\left\langle\left(1,0, \frac{\partial h^{+}}{\partial u}\right),\left(0,1, \frac{\partial h^{+}}{\partial v}\right)\right\rangle=k_{1} k_{3}^{+} u^{2}+\left(\left(k_{3}^{+}\right)^{2}+k_{1} k_{2}\right) u v+ \\
& +k_{2} k_{3}^{+} v^{2}+\left(\frac{1}{2} k_{1} c+\frac{1}{2} k_{3}^{+} a\right) u^{3}+\left(\frac{3}{2} k_{3}^{+} c+k_{1} b+\frac{1}{2} k_{2} a\right) u^{2} v+ \\
& +\left(\frac{3}{2} k_{3}^{+} b+\frac{1}{2} k_{1} d+k_{2} c\right) u v^{2}+\left(\frac{1}{2} k_{3}^{+} d+\frac{1}{2} k_{2} b\right) v^{3}+O(4) \\
G & =\left\langle\frac{\partial X^{+}}{\partial v}, \frac{\partial X^{+}}{\partial v}\right\rangle=\left\langle\left(0,1, \frac{\partial h^{+}}{\partial v}\right),\left(0,1, \frac{\partial h^{+}}{\partial v}\right)\right\rangle=1+k_{3}^{2} u^{2}+2 k_{2} k_{3} u v+ \\
& +k_{2}^{2} v^{2}+k_{3}^{+} c u^{3}+\left(k_{3}^{+} d+2 k_{2} b\right) u v^{2}+\left(2 k_{3}^{+} b+k_{2} c\right) u^{2} v+k_{2} d v^{3}+O(4)
\end{aligned}
$$

Os coeficientes da Segunda Forma Fundamental de $X^{+}$expressos na carta $(u, v)$ são dados por $\frac{e}{\left|\mathcal{N}^{+}\right|}, \frac{f}{\left|\mathcal{N}^{+}\right|}$e $\frac{g}{\left|\mathcal{N}^{+}\right|}$, onde $\mathcal{N}^{+}$é o vetor normal definido em 2.5 e

$$
\begin{aligned}
& e=\left\langle\frac{\partial^{2} X^{+}}{\partial u^{2}}, \mathcal{N}^{+}\right\rangle=k_{1}+a u+c v+\frac{1}{2} A u^{2}+B u v+\frac{1}{2} C v^{2}+O(3), \\
& f=\left\langle\frac{\partial^{2} X^{+}}{\partial u \partial v}, \mathcal{N}^{+}\right\rangle=k_{3}^{+}+c u+b v+\frac{1}{2} B u^{2}+C u v+\frac{1}{2} D v^{2}+O(3), \\
& g=\left\langle\frac{\partial^{2} X^{+}}{\partial v^{2}}, \mathcal{N}^{+}\right\rangle=k_{2}^{+}+b u+d v+\frac{1}{2} C u^{2}+D u v+\frac{1}{2} E v^{2}+O(3)
\end{aligned}
$$

Para a superfície $\mathbf{S}^{-}$, considerando a parametrização $X^{-}(u, v)=\left(u, v, h^{-}(u, v)\right)$ e sendo $h^{-}$como em (2.4) temos:

$$
\begin{aligned}
\frac{\partial h^{-}}{\partial u} & =k_{1} u+k_{3}^{-} v+\frac{1}{2} a u^{2}+\gamma u v+\frac{1}{2} \beta v^{2}+\frac{1}{6} A u^{3}+\frac{1}{2} B_{1} u^{2} v+\frac{1}{2} C_{1} u v^{2}+\frac{1}{6} D_{1} v^{3}+O(4), \\
\frac{\partial h^{-}}{\partial v} & =k_{3}^{-} u+k_{2}^{-} v+\frac{1}{2} \gamma u^{2}+\beta u v+\frac{1}{2} \rho v^{2}+\frac{1}{6} B_{1} u^{3}+\frac{1}{2} C_{1} u^{2} v+\frac{1}{2} D_{1} u v^{2}+\frac{1}{6} E_{1} v^{3}+O(4), \\
\frac{\partial^{2} h^{-}}{\partial u^{2}} & =K_{1}+a u+\gamma v+\frac{1}{2} A u^{2}+B_{1} u v+\frac{1}{2} C_{1} v^{2}+O(3), \\
\frac{\partial^{2} h^{-}}{\partial u \partial v} & =k_{3}^{-}+\gamma u+\beta v+\frac{1}{2} B_{1} u^{2}+C_{1} u v+\frac{1}{2} D_{1} v^{2}+O(3), \\
\frac{\partial^{2} h^{-}}{\partial v^{2}} & =k_{2}^{-}+\beta u+\rho v+\frac{1}{2} C_{1} u^{2}+D_{1} u v+\frac{1}{2} E_{1} v^{2}+O(3) .
\end{aligned}
$$

O vetor normal (não unitário) a $\mathbf{S}^{-}$é dado por: 


$$
\mathcal{N}^{-}(u, v)=\left(\frac{\partial X^{-}}{\partial u} \wedge \frac{\partial X^{-}}{\partial v}\right)(u, v)=\left(-\frac{\partial h^{-}}{\partial u},-\frac{\partial h^{-}}{\partial v}, 1\right) .
$$

Os coeficientes da Primeira Forma Fundamental de $X^{-}$expressos na carta $(u, v)$ são:

$$
\begin{aligned}
E & =\left\langle\frac{\partial X^{-}}{\partial u}, \frac{\partial X^{-}}{\partial u}\right\rangle=\left\langle\left(1,0, \frac{\partial h^{-}}{\partial u}\right),\left(1,0, \frac{\partial h^{-}}{\partial u}\right)\right\rangle=1+\lambda^{2} u^{2}+2 \lambda k_{3}^{+} u v+ \\
& +\left(k_{3}^{+}\right)^{2} v^{2}+k_{1} a u^{3}+\left(2 k_{3}^{-} \gamma+k_{1} \beta\right) u v^{2}+\left(2 k_{1} \gamma+k_{3}^{-} \alpha\right) u^{2} v+k_{3}^{-} \beta v^{3}+O(4), \\
F & =\left\langle\frac{\partial X^{-}}{\partial u}, \frac{\partial X^{-}}{\partial v}\right\rangle=\left\langle\left(1,0, \frac{\partial h^{-}}{\partial u}\right),\left(0,1, \frac{\partial h^{-}}{\partial v}\right)\right\rangle=\lambda k_{3}^{-} u^{2}+\left(\left(k_{3}^{-}\right)^{2}+k_{1} k_{2}^{-}\right) u v+ \\
& +k_{2}^{-} k_{3}^{-} v^{2}+\left(\frac{1}{2} k_{1} \gamma+\frac{1}{2} k_{3}^{-} a\right) u^{3}+\left(\frac{3}{2} k_{3}^{-} \gamma+k_{1} \beta+\frac{1}{2} k_{2}^{-} a\right) u^{2} v+ \\
& +\left(\frac{3}{2} k_{3}^{-} \beta+\frac{1}{2} k_{1} \rho+k_{2}^{-} \gamma\right) u v^{2}+\left(\frac{1}{2} k_{3}^{-} \rho+\frac{1}{2} k_{2}^{-} \beta\right) v^{3}+O(4), \\
G & =\left\langle\frac{\partial X^{-}}{\partial v}, \frac{\partial X^{-}}{\partial v}\right\rangle=\left\langle\left(0,1, \frac{\partial h^{-}}{\partial v}\right),\left(0,1, \frac{\partial h^{-}}{\partial v}\right)\right\rangle=1+\left(k_{3}^{-}\right)^{2} u^{2}+2 k_{2}^{-} k_{3}^{-} u v+ \\
& +\left(k_{2}^{-}\right)^{2} v^{2}+k_{3}^{-} \gamma u^{3}+\left(k_{3}^{-} \rho+2 k_{2}^{-} \beta\right) u v^{2}+\left(2 k_{3}^{-} \beta+k_{2}^{-} \gamma\right) u^{2} v+k_{2}^{-} \rho v^{3}+O(4) .
\end{aligned}
$$

Os coeficientes da Segunda Forma Fundamental de $X^{-}$expressos na carta $(u, v)$ são dados por $\frac{e}{\left|\mathcal{N}^{-}\right|}, \frac{f}{\left|\mathcal{N}^{-}\right|}$e $\frac{g}{\left|\mathcal{N}^{-}\right|}$, onde $\mathcal{N}^{-}$é o vetor normal definido em $(2.8)$ e

$$
\begin{aligned}
& e=\left\langle\frac{\partial^{2} X^{-}}{\partial u^{2}}, \mathcal{N}^{-}\right\rangle=k_{1}+a u+\gamma v+\frac{1}{2} A u^{2}+B_{1} u v+\frac{1}{2} C_{1} v^{2}+O(3), \\
& f=\left\langle\frac{\partial^{2} X^{-}}{\partial u \partial v}, \mathcal{N}^{-}\right\rangle=k_{3}^{-}+\gamma u+\beta v+\frac{1}{2} B_{1} u^{2}+C_{1} u v+\frac{1}{2} D_{1} v^{2}+O(3), \\
& g=\left\langle\frac{\partial^{2} X^{-}}{\partial v^{2}}, \mathcal{N}^{-}\right\rangle=k_{2}^{-}+\beta u+\rho v+\frac{1}{2} C_{1} u^{2}+D_{1} u v+\frac{1}{2} E_{1} v^{2}+O(3) .
\end{aligned}
$$

A proposição 2.1.1, abaixo, estabelece as condições para que as linhas de curvatura principal da superfície $\mathbf{S}^{+}$sejam tangentes quadraticamente ou cruzem transversalmente o bordo $\boldsymbol{\delta}$ na vizinhança da origem. 
Proposição 2.1.1. Considere a superfície $\mathbf{S}^{+}$parametrizada, em uma vizinhança da origem, por $X^{+}(u, v)=\left(u, v, h^{+}(u, v)\right)$, sendo $h^{+}(u, v)=\frac{1}{2} k_{1} u^{2}+k_{3}^{+} u v+\frac{1}{2} k_{2}^{+} v^{2}+\frac{1}{6} a u^{3}+$ $+\frac{1}{2} b u v^{2}+\frac{1}{2} c u^{2} v+\frac{1}{6} d v^{3}+O(4)$, e a curva bordo parametrizada por $\delta$. Então, as linhas de curvatura principal de $\mathbf{S}^{+}$são transversais a $\delta$ na origem $\delta(0)$ se, e somente se, $k_{3}^{+} \neq 0$. Supondo que $k_{2}^{+}<k_{1}$, uma linha de curvatura principal máxima tem contato quadrático com $\boldsymbol{\delta}$ em $\delta(0)$ se, e somente se, $k_{3}^{+}=0$ e $c \neq 0$. Este contato é interno (respectivamente externo) se $c>0$ (respectivamente $c<0$ ). Veja figura 2.1.

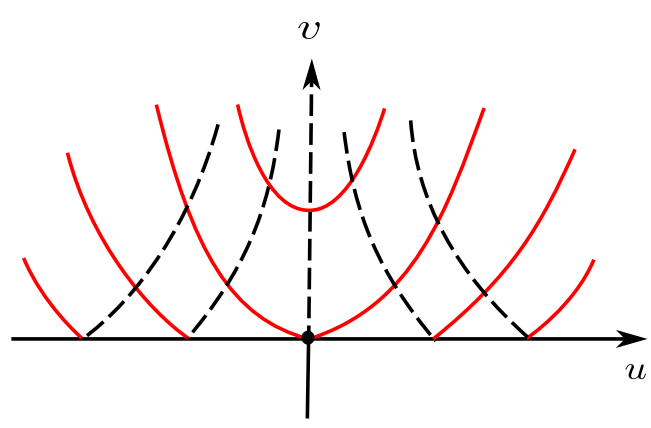

$c>0$

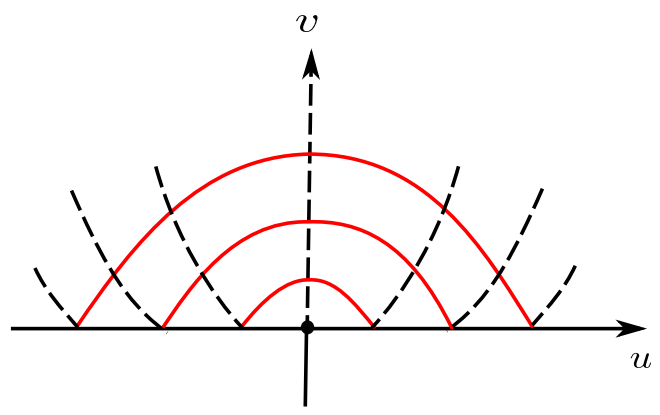

$c<0$

Figura 2.1: Configuração principal de $\mathbf{S}^{+}$em uma vizinhança da origem: tangência interna para $c>0$ e externa para $c<0$. Supondo-se $k_{2}^{+}<k_{1}$, temos que as linhas tracejadas (respectivamente contínuas) ilustram as linhas de curvatura principal mínima (respectivamente máxima).

Demonstração. Seja $X^{+}(u, v)=\left(u, v, h^{+}(u, v)\right)$ a parametrização da superfície $\mathbf{S}^{+}$, onde

$$
\begin{aligned}
h^{+}(u, v) & =\frac{1}{2} k_{1} u^{2}+k_{3}^{+} u v+\frac{1}{2} k_{2}^{+} v^{2}+\frac{1}{6} a u^{3}+\frac{1}{2} b u v^{2}+\frac{1}{2} c u^{2} v+\frac{1}{6} d v^{3}+ \\
& +\frac{1}{24} A u^{4}+\frac{1}{6} B u^{3} v+\frac{1}{4} C u^{2} v^{2}+\frac{1}{6} D u v^{3}+\frac{1}{24} E v^{4}+O(5) .
\end{aligned}
$$

Lembremos que a equação diferencial das linhas de curvatura é dada por:

$$
(F g-G f) d v^{2}+(E g-G e) d u d v+(E f-F e) d u^{2}=0 .
$$

Das expressões (2.6) e 2.7) obtemos que os coeficientes da Primeira e Segunda Formas Fundamentais na origem $(0,0)$ são:

$$
\begin{aligned}
E(0,0) & =1, & F(0,0) & =0, & G(0,0) & =1, \\
e(0,0) & =k_{1}, & f(0,0) & =k_{3}^{+}, & g(0,0) & =k_{2}^{+} .
\end{aligned}
$$

Assim, as direções principais de $\mathbf{S}^{+}$em $(0,0)$ satisfazem

$$
\left(-k_{3}^{+}\right) d v^{2}+\left(k_{2}^{+}-k_{1}\right) d u d v+\left(k_{3}^{+}\right) d u^{2}=0 .
$$


e o vetor $T(0)$ é uma direção principal de $(2.12)$ se, e somente se, $k_{3}^{+}=0(T(u)$ é o vetor tangente unitário da curva $u \longmapsto \delta(u))$.

Próximo da origem, a equação 2.11 torna-se:

$$
\begin{aligned}
& {\left[-c u-b v-\frac{1}{2} B u^{2}+\left(-C+\left(k_{2}^{+}\right)^{2} k_{1}\right) u v-\frac{1}{2} D v^{2}+O(3)\right] d v^{2}+\left[k_{2}^{+}-k_{1}+\right.} \\
& +(b-a) u+(d-c) v+\left(\frac{1}{2} C+k_{1}^{2} k_{2}^{+}-\frac{1}{2} A\right) u^{2}+(D-B) u v+ \\
& \left.+\left(\frac{1}{2} E-\left(k_{2}^{+}\right)^{2} k_{1}-\frac{1}{2} C\right) v^{2}+O(3)\right] d u d v+\left[c u+b v+\frac{1}{2} B u^{2}+\right. \\
& \left.+\left(C-k_{1}^{2} k_{2}^{+}\right) u v+\frac{1}{2} D v^{2}+O(3)\right] d u^{2}=0 .
\end{aligned}
$$

Usando o "Método dos Coeficientes a Determinar", temos que na vizinhança da origem, as linhas de curvatura principal máxima são soluções de (2.13) e descritas por:

$$
v(u)=\frac{c}{2\left(k_{1}-k_{2}^{+}\right)} u^{2}+\frac{c(3 b-2 a)+B\left(k_{1}-k_{2}^{+}\right)}{6\left(k_{1}-k_{2}^{+}\right)^{2}} u^{3}+O(4), \quad v \geq 0 .
$$

Isto completa a demonstração.

Observação 2.1.2. Na proposição 2.1.1 mudamos a orientação de $\mathbf{S}^{+}$se considerarmos $k_{2}^{+}>k_{1}$. Logo, supondo que $k_{3}^{+}=0$, uma linha de curvatura principal mínima terá contato quadrático com $\boldsymbol{\delta}$ em $\delta(0)$ interno (respectivamente externo) se $c<0$ (respectivamente $c>0)$.

A proposição seguinte é a análoga da proposição 2.1.1 para a superfície $\mathbf{S}^{-}$.

Proposição 2.1.3. Considere a superfície $\mathbf{S}^{-}$parametrizada, em uma vizinhança da origem, por $X^{-}(u, v)=\left(u, v, h^{-}(u, v)\right)$, sendo $h^{-}(u, v)=\frac{1}{2} k_{1} u^{2}+k_{3}^{-} u v+\frac{1}{2} k_{2}^{-} v^{2}+\frac{1}{6} a u^{3}+$ $+\frac{1}{2} \beta u v^{2}+\frac{1}{2} \gamma u^{2} v+\frac{1}{6} \rho v^{3}+O(4)$, e a curva bordo parametrizada por $\delta$. Então, as linhas de curvatura principal de $\mathbf{S}^{-}$são transversais a $\delta$ na origem $\delta(0)$ se, e somente se, $k_{3}^{-} \neq 0$. Supondo que $k_{1}<k_{2}^{-}$, uma linha de curvatura principal mínima tem contato quadrático com $\boldsymbol{\delta}$ em $\delta(0)$ se, e somente se, $k_{3}^{-}=0$ e $\gamma \neq 0$. Este contato é interno (respectivamente externo) se $\gamma>0$ (respectivamente $\gamma<0$ ). Veja figura 2.2.

Demonstração. Seja $X^{-}(u, v)=\left(u, v, h^{-}(u, v)\right)$ a parametrização da superfície $\mathbf{S}^{-}$, onde

$$
\begin{aligned}
h^{-}(u, v) & =\frac{1}{2} k_{1} u^{2}+k_{3}^{-} u v+\frac{1}{2} k_{2}^{-} v^{2}+\frac{1}{6} a u^{3}+\frac{1}{2} \beta u v^{2}+\frac{1}{2} \gamma u^{2} v+\frac{1}{6} \rho v^{3}+ \\
& +\frac{1}{24} A u^{4}+\frac{1}{6} B_{1} u^{3} v+\frac{1}{4} C_{1} u^{2} v^{2}+\frac{1}{6} D_{1} u v^{3}+\frac{1}{24} E_{1} v^{4}+O(5) .
\end{aligned}
$$



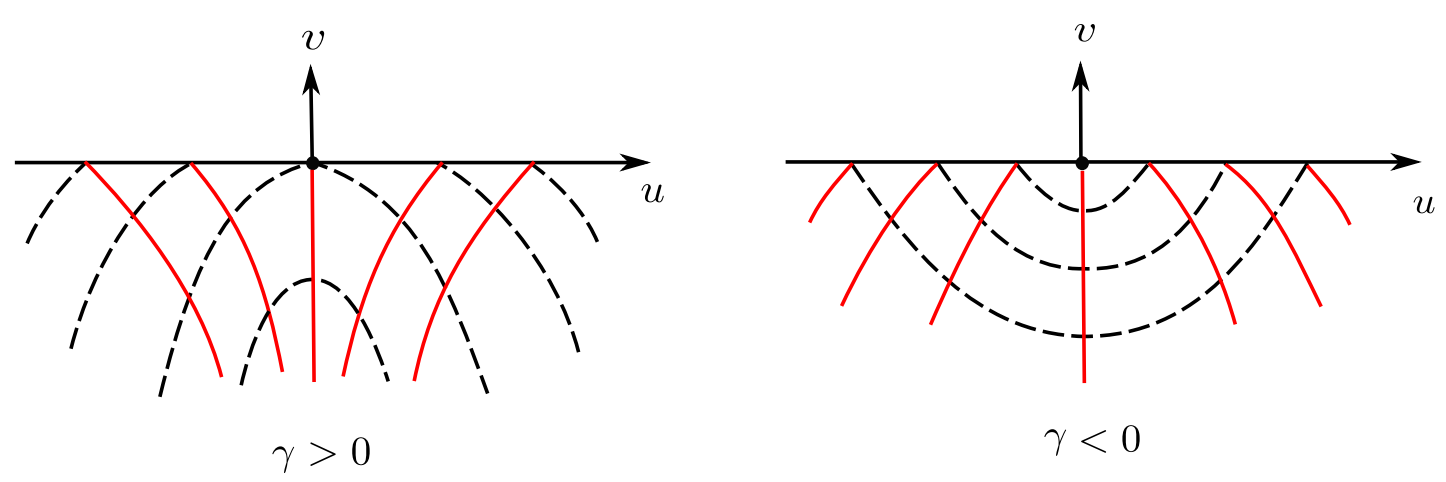

Figura 2.2: Configuração principal de $\mathbf{S}^{-}$em uma vizinhança da origem: tangência interna para $\gamma>0$ e externa para $\gamma<0$. Supondo-se $k_{1}<k_{2}^{-}$, temos que as linhas tracejadas (respectivamente contínuas) ilustram as linhas de curvatura principal mínima (respectivamente máxima).

Das expressões 2.9 e 2.10 obtemos que os coeficientes da Primeira e Segunda Formas Fundamentais na origem $(0,0)$ são dados por:

$$
\begin{aligned}
E(0,0) & =1, & F(0,0) & =0, & G(0,0) & =1, \\
e(0,0) & =k_{1}, & f(0,0) & =k_{3}^{-}, & g(0,0) & =k_{2}^{-} .
\end{aligned}
$$

Assim, as direções principais de $\mathbf{S}^{-}$em $(0,0)$ satisfazem

$$
\left(-k_{3}^{-}\right) d v^{2}+\left(k_{2}^{-}-k_{1}\right) d u d v+\left(k_{3}^{-}\right) d u^{2}=0 .
$$

e o vetor $T(0)$ é uma direção principal de 2.14 se, e somente se, $k_{3}^{-}=0(T(u)$ é o vetor tangente unitário da curva $u \longmapsto \delta(u))$.

Próximo da origem, a equação 2.11 torna-se:

$$
\begin{aligned}
& {\left[-\gamma u-\beta v-\frac{1}{2} B_{1} u^{2}+\left(-C_{1}+\left(k_{2}^{-}\right)^{2} k_{1}\right) u v-\frac{1}{2} D_{1} v^{2}+O(3)\right] d v^{2}+\left[k_{2}^{-}-k_{1}+\right.} \\
& +(\beta-a) u+(\rho-\gamma) v+\left(\frac{1}{2} C_{1}+k_{1}^{2} k_{2}^{-}-\frac{1}{2} A\right) u^{2}+\left(D_{1}-B_{1}\right) u v+ \\
& \left.+\left(\frac{1}{2} E-\left(k_{2}^{-}\right)^{2} k_{1}-\frac{1}{2} C\right) v^{2}+O(3)\right] d u d v+\left[\gamma u+\beta v+\frac{1}{2} B_{1} u^{2}+\right. \\
& \left.+\left(C_{1}-k_{1}^{2} k_{2}^{-}\right) u v+\frac{1}{2} D_{1} v^{2}+O(3)\right] d u^{2}=0
\end{aligned}
$$

Pelo "Método dos Coeficientes a Determinar" temos que na vizinhança da origem as linhas de curvatura principal mínima são soluções de 2.15 e descritas por:

$$
v(u)=\frac{\gamma}{2\left(k_{1}-k_{2}^{-}\right)} u^{2}+\frac{\gamma(3 \beta-2 a)+B_{1}\left(k_{1}-k_{2}^{-}\right)}{6\left(k_{1}-k_{2}^{-}\right)^{2}} u^{3}+O(4), \quad v \leq 0 .
$$

Isto completa a demonstração. 
Observação 2.1.4. Na proposição 2.1.3 mudamos a orientação de $\mathbf{S}^{-}$se considerarmos $k_{1}>k_{2}^{-}$. Logo, supondo que $k_{3}^{-}=0$, uma linha de curvatura principal máxima terá contato quadrático com $\boldsymbol{\delta}$ em $\delta(0)$ interno (respectivamente externo) se $\gamma<0$ (respectivamente $\gamma>0)$

Considere $\mathbf{S}=\mathbf{S}^{+} \cup \boldsymbol{\delta} \cup \mathbf{S}^{-}$como sendo a superfície sem bordo obtida pela justaposição de $\mathbf{S}^{+}$e $\mathbf{S}^{-}$ao longo do bordo $\boldsymbol{\delta}$. Observe que $\mathbf{S}$ é suave no complementar da curva bordo comum $\boldsymbol{\delta}$, de classe $C^{0}$ ao longo de $\boldsymbol{\delta}$ e diferenciável, isto é, tem plano tangente, apenas na origem $\delta(0)$, onde $\boldsymbol{\delta}=\{\delta(u), u \in \mathbb{R}\}$. A curva $\boldsymbol{\delta}$ será também chamada curva de descontinuidade da superfície $\mathbf{S}$.

Supondo que $k_{3}^{+}=k_{3}^{-}=0$, as folheações principais de $\mathbf{S}^{+}$e $\mathbf{S}^{-}$, em uma vizinhança da origem, são descritas pelas proposições 2.1.1 e 2.1.3, respectivamente (veja figuras 2.1 e 2.2 ). As figuras 2.3 e 2.4 ilustram as possíveis configurações topológicas de $\mathbf{S}$.

Veremos que, para uma função de regularização específica, a configuração principal suave por partes é deformada em uma suave na qual surgem pontos umbílicos. As tangências quadráticas das folhas suaves por partes da folheação principal da superfície $\mathbf{S}=\mathbf{S}^{+} \cup \boldsymbol{\delta} \cup \mathbf{S}^{-}$darão lugar a configurações principais locais equivalentes àquelas das vizinhanças dos umbílicos Darbouxianos dos tipos $D_{1}$ e $D_{3}$.

Sob certas condições, estabelecidas no teorema 2.2.6, após a regularização da superfície $\mathbf{S}$, na figura 2.3 a configuração principal de $(i)$ será equivalente à do umbílico $D_{3}$, as configurações principais de $(i i)$ e de (iii) serão equivalentes às dos umbílicos $D_{1}$ e $D_{3}$ e a configuração principal de $(i v)$ será equivalente à do umbílico $D_{1}$. Por outro lado, as configurações principais da figura 2.4 não originarão umbílicos após a regularização (veja observação 2.2.5. 
As figuras 2.3 e 2.4 abaixo ilustram as possíveis configurações topológicas de $\mathbf{S}:{ }^{2}$

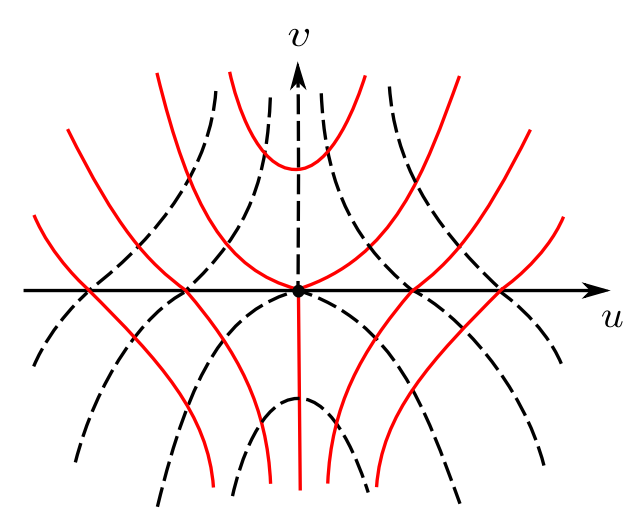

(i) $\quad c>0$ e $\gamma>0$

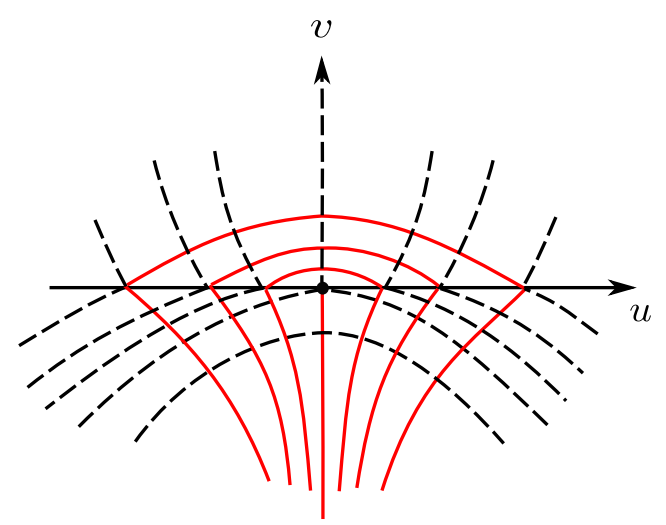

(iii) $\quad c<0$ e $\gamma>0$

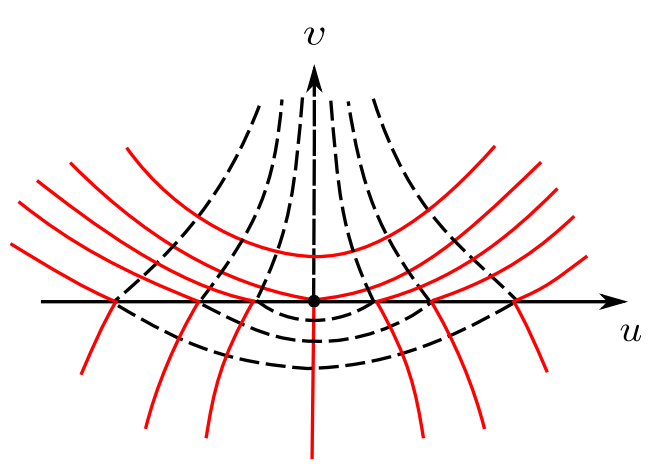

(ii) $\quad c>0$ e $\gamma<0$

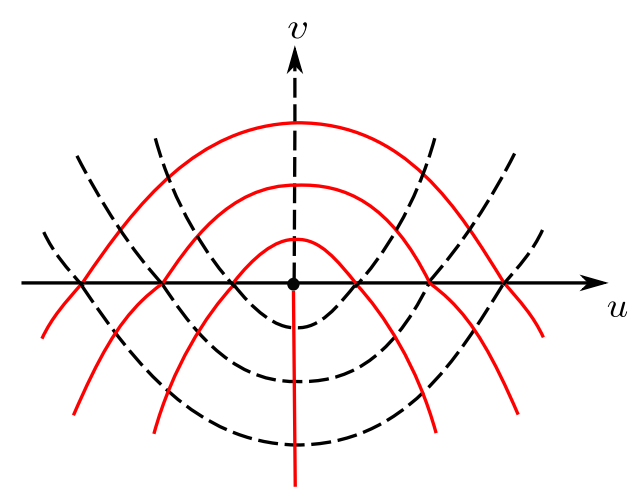

(iv) $\quad c<0$ e $\gamma<0$

Figura 2.3: Configuraçôes principais suaves por partes de $\mathbf{S}=\mathbf{S}^{+} \cup \boldsymbol{\delta} \cup \mathbf{S}^{-}$em uma vizinhança da origem considerando $k_{2}^{+}<k_{1}<k_{2}^{-}$. Linhas tracejadas (respectivamente contínuas) ilustram as linhas de curvatura principal mínima (respectivamente máxima).

${ }^{2}$ Para esboçar as linhas de curvatura de $\mathbf{S}$ basta analisar o sinal de $c$ na proposição 2.1.1 e de $\gamma$ na proposição 2.1.3 observando as possibilidades $\left(k_{1}<k_{2}^{+}\right.$e $\left.k_{1}<k_{2}^{-}\right)$e $k_{2}^{+}<k_{1}<k_{2}^{-}$. 


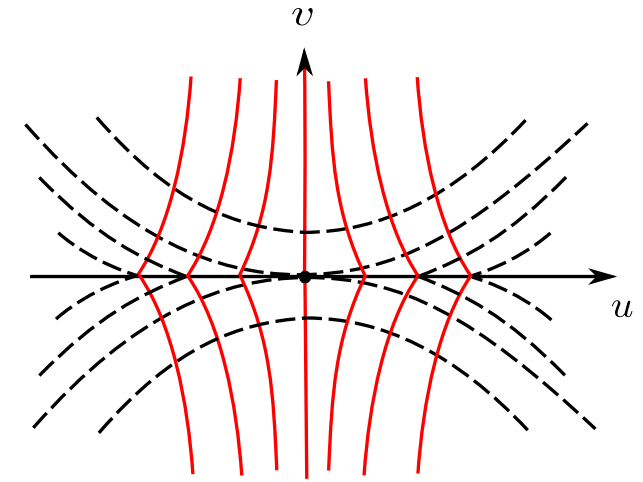

(i) $\quad c<0$ e $\gamma>0$

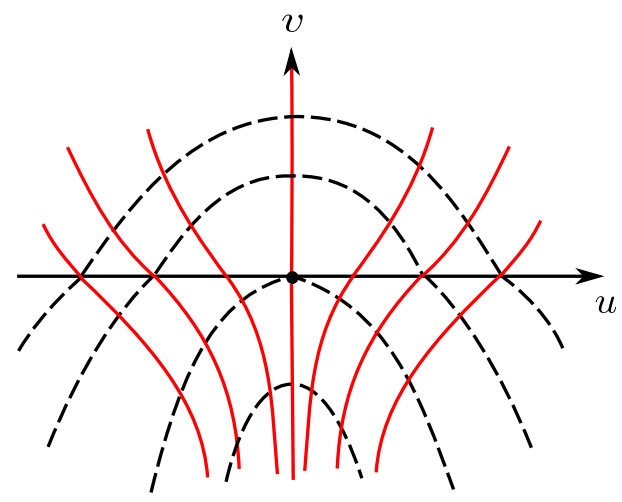

(iii) $\quad c>0$ e $\gamma>0$

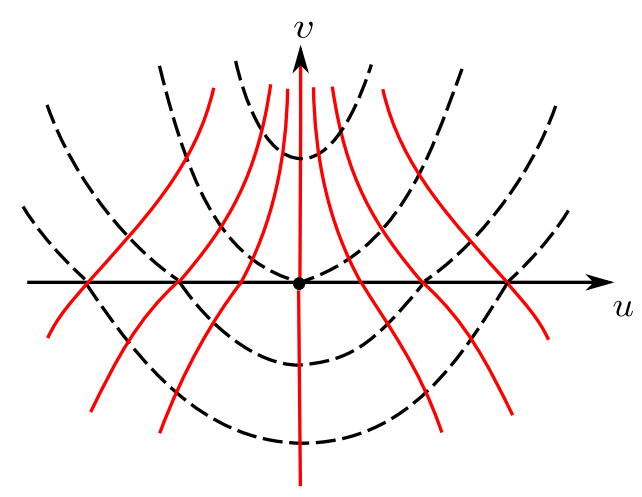

(ii) $\quad c<0$ e $\gamma<0$

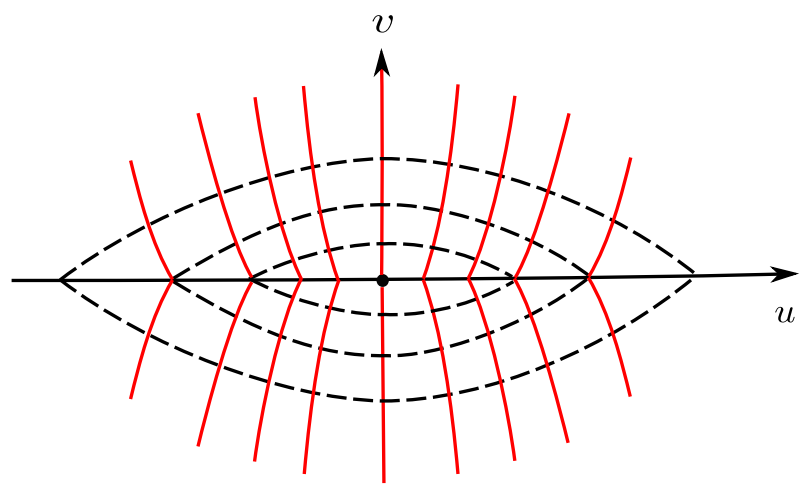

(iv) $\quad c>0$ e $\gamma<0$

Figura 2.4: Configurações principais suaves por partes de $\mathbf{S}=\mathbf{S}^{+} \cup \boldsymbol{\delta} \cup \mathbf{S}^{-}$em uma vizinhança da origem considerando $k_{1}<k_{2}^{+}$e $k_{1}<k_{2}^{-}$. Linhas tracejadas (respectivamente contínuas) ilustram as linhas de curvatura principal mínima (respectivamente máxima).

\subsection{2 $2^{\circ}$ Caso: Ponto de Cruzamento Transversal}

Seja $\mathcal{C}$ o conjunto dos pontos sobre $\boldsymbol{\delta}$ de singularidades tangenciais. Se $\delta\left(u_{0}\right) \in \boldsymbol{\delta} \backslash \mathcal{C}$, temos que as linhas de curvaturas principais das superfícies $\mathbf{S}^{+}$e $\mathbf{S}^{-}$são transversais ao bordo comum $\delta$ em $\delta\left(u_{0}\right)$. Portanto, fica bem definido o ângulo $w\left(u_{0}\right) \in(0, \pi)$ entre a linha de curvatura principal mínima da superfície $\mathbf{S}^{+}$e a linha de curvatura principal mínima da superfície $\mathbf{S}^{-}$. Considere também $\theta^{+}\left(u_{0}\right)$ (respectivamente $\theta^{-}\left(u_{0}\right)$ ) como sendo o ângulo entre o eixo- $u$ e a linha principal mínima da superfície $\mathbf{S}^{+}$(respectivamente $\mathbf{S}^{-}$) transversal à curva $\boldsymbol{\delta}$ em $\delta\left(u_{0}\right)$ (veja figuras 2.5, 2.6, 2.7 e 2.8. . Neste contexto, podemos introduzir as seguintes definições 2.1.5 e 2.1.6, abaixo:

Definição 2.1.5. Seja $\delta\left(u_{0}\right) \in \boldsymbol{\delta} \backslash \mathcal{C}$ e suponha $\theta^{+}\left(u_{0}\right) \in\left[0, \frac{\pi}{2}\right)$ e $\theta^{-}\left(u_{0}\right) \in\left[0,-\frac{\pi}{2}\right)$. O ponto $\delta\left(u_{0}\right)$ será chamado:

(i) um ponto de deslizamento se $\omega\left(u_{0}\right)=\left(\theta^{+}-\theta^{-}\right)\left(u_{0}\right) \in\left[0, \frac{\pi}{2}\right)$, ou equivalentemente, se $\tan \left(\theta^{+}\left(u_{0}\right)\right) \cdot \tan \left(\theta^{-}\left(u_{0}\right)\right)>-1$ (figura 2.5); 


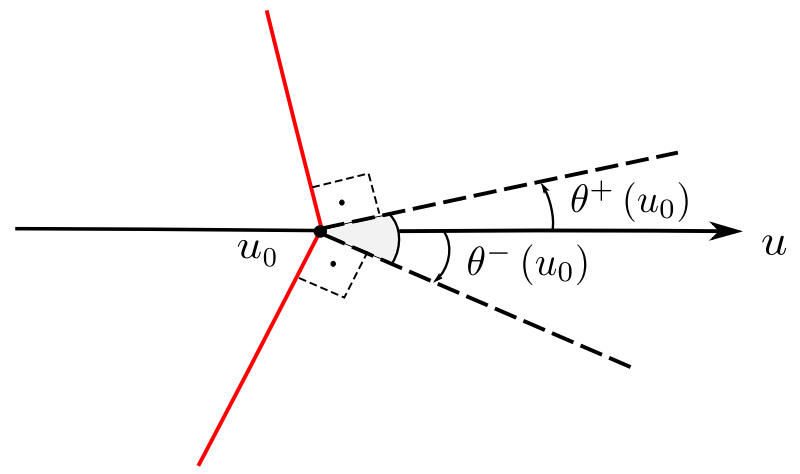

Figura 2.5: $\delta\left(u_{0}\right)$ é um ponto de deslizamento para as linhas de curvatura principais mínimas (linhas tracejadas) das superfícies $\mathbf{S}^{+}$e $\mathbf{S}^{-}$.

(ii) um ponto de costura se $\omega\left(u_{0}\right)=\left(\theta^{+}-\theta^{-}\right)\left(u_{0}\right) \in\left(\frac{\pi}{2}, \pi\right)$, ou equivalentemente, se $\tan \left(\theta^{+}\left(u_{0}\right)\right) \cdot \tan \left(\theta^{-}\left(u_{0}\right)\right)<-1$ (figura 2.6);

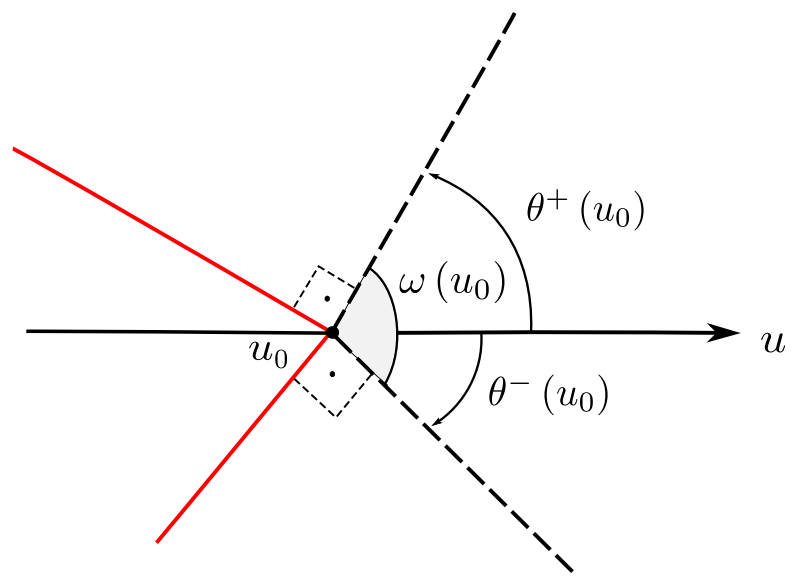

Figura 2.6: $\delta\left(u_{0}\right)$ é um ponto de costura para as linhas de curvatura principais mínimas (linhas tracejadas) das superfícies $\mathbf{S}^{+} e \mathbf{S}^{-}$.

(iii) um ponto de transição se $\omega\left(u_{0}\right)=\left(\theta^{+}-\theta^{-}\right)\left(u_{0}\right)=\frac{\pi}{2}$, ou equivalentemente, se $\tan \left(\theta^{+}\left(u_{0}\right)\right) \cdot \tan \left(\theta^{-}\left(u_{0}\right)\right)=-1$ (figura 2.7).

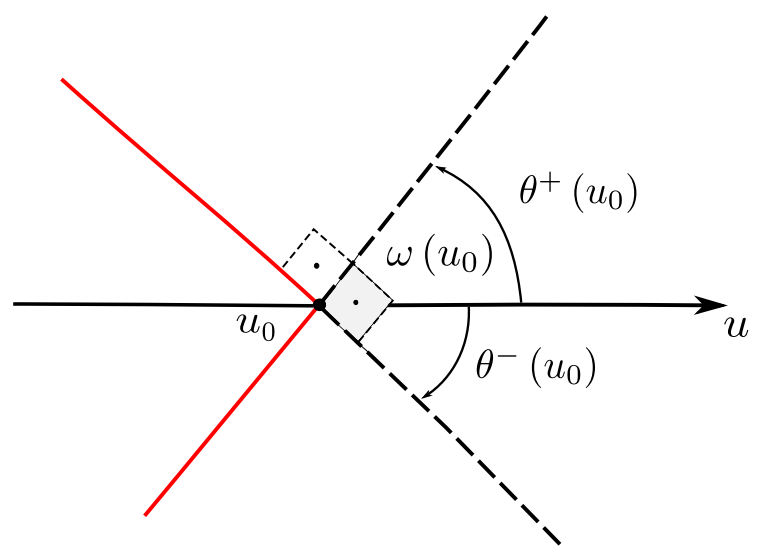

Figura 2.7: $\delta\left(u_{0}\right)$ é um ponto de transição para as linhas de curvatura principais mínimas (linhas tracejadas) e máximas (linhas contínuas) das superfícies $\mathbf{S}^{+} e \mathbf{S}^{-}$. 
Definição 2.1.6. Seja $\delta\left(u_{0}\right) \in \boldsymbol{\delta} \backslash \mathcal{C}$. O ponto $\delta\left(u_{0}\right)$ será também chamado um ponto de costura se $\omega\left(u_{0}\right)=\left(\theta^{+}-\theta^{-}\right)\left(u_{0}\right) \in\left[0, \frac{\pi}{2}\right) \cup\left(\frac{\pi}{2}, \pi\right]$ e $\theta^{+}\left(u_{0}\right) \in\left[\frac{\pi}{2}, \pi\right]$ (figura 2.8. esquerda) ou se $\omega\left(u_{0}\right) \in\left[0, \frac{\pi}{2}\right) \cup\left(\frac{\pi}{2}, \pi\right]$ e $\theta^{-}\left(u_{0}\right) \in\left[-\frac{\pi}{2},-\pi\right]$ (figura 2.8, direita).
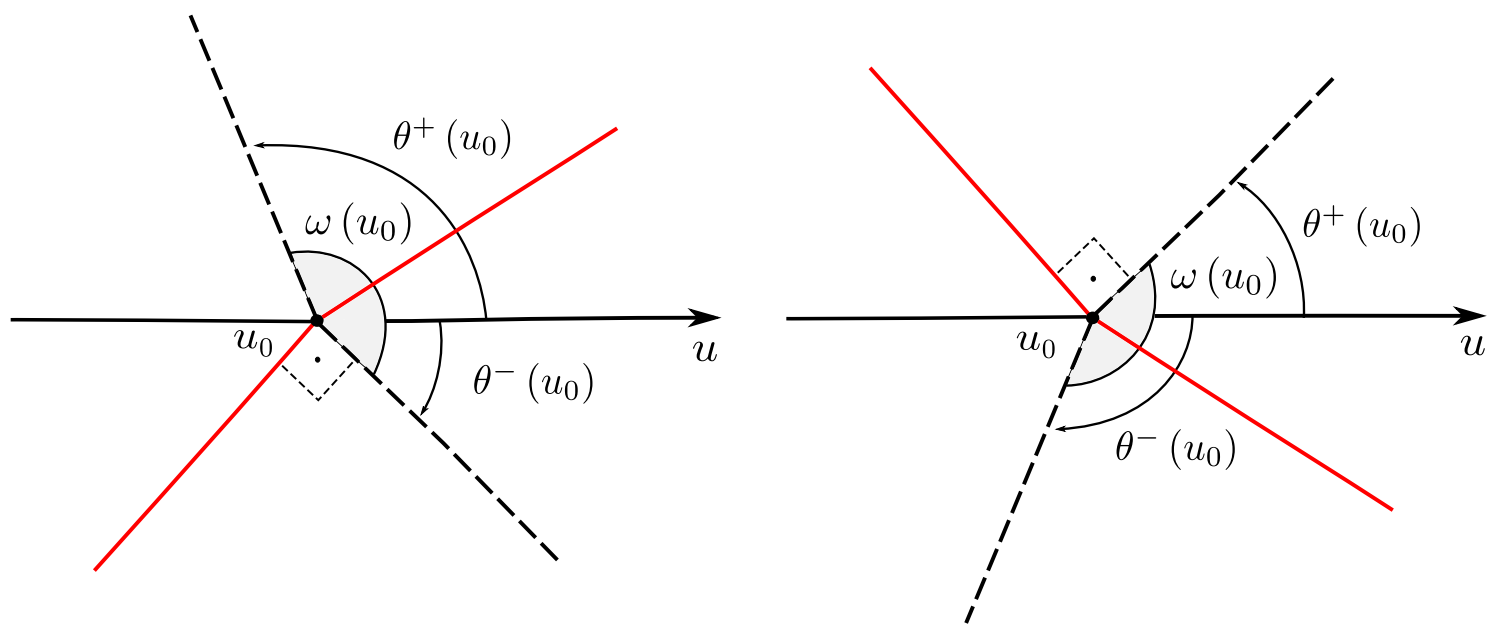

Figura 2.8: $\delta\left(u_{0}\right)$ é um ponto de costura para as linhas de curvatura principais mínimas (linhas tracejadas) das superfícies $\mathbf{S}^{+} e \mathbf{S}^{-}$.

A nomenclatura das definições 2.1.5 e 2.1.6, acima, é a mesma adotada por Filippov em [3] e nos trabalhos de Sotomayor e Teixeira [12] e Sotomayor e Machado [11].

Observação 2.1.7. Como as linhas de curvatura principal de uma superfície encontramse ortogonalmente segue, pela definição 2.1.5, que se $\delta\left(u_{0}\right)$ é um ponto de costura (respectivamente deslizamento) para as linhas principais minimas de $\mathbf{S}^{+}$e de $\mathbf{S}^{-}$, então $\delta\left(u_{0}\right)$ será um ponto de deslizamento (respectivamente costura) para as linhas principais máximas de $\mathbf{S}^{+}$e de $\mathbf{S}^{-}$. Já o ponto de costura da definição 2.1.6 é ponto de costura tanto para as linhas de curvatura principais minimas quanto para as linhas de curvatura principais máximas das superfícies $\mathbf{S}^{+}$e $\mathbf{S}^{-}$.

Vimos na proposição 2.1.1 (respectivamente 2.1.3) que as linhas de curvatura principal da superfície $\mathbf{S}^{+}$(respectivamente $\mathbf{S}^{-}$) são transversais ao bordo comum $\boldsymbol{\delta}$ em uma vizinhança da origem $\delta(0)$ se, e somente se, $k_{3}^{+} \neq 0$ (respectivamente $k_{3}^{-} \neq 0$ ).

Suponhamos que $k_{3}^{+} \cdot k_{3}^{-} \neq 0$. Usando a mesma notação das definições 2.1.5 e 2.1.6. vamos supor $u_{0}=0$ e $\theta_{0}^{+}:=\theta^{+}(0)$ (respectivamente $\theta_{0}^{-}:=\theta^{-}(0)$ ) o ângulo entre o eixo- $u$ e a linha principal mínima da superfície $\mathbf{S}^{+}$(respectivamente $\mathbf{S}^{-}$) transversal à curva $\boldsymbol{\delta}$ na origem (veja figura 2.9).

Uma condição necessária para a existência de pontos umbílicos sobre a regularização da superfície $\mathbf{S}$ é que tenhamos sobre $\boldsymbol{\delta}$ um ponto de transição. Neste ponto, por definição, as linhas de curvatura principais mínimas (e máximas) de $\mathbf{S}^{+}$e $\mathbf{S}^{-}$são ortogonais. 
A proposição 2.1.8, abaixo, estabelece as condições para que a origem $\delta(0)$ seja um ponto de transição.

Proposição 2.1.8. Sob as mesmas condições acima, considere a superfície $\mathbf{S}=\mathbf{S}^{+} \cup \boldsymbol{\delta} \cup \mathbf{S}^{-}$e suponha que $k_{3}^{+} \cdot k_{3}^{-} \neq 0$. Então,

$$
\delta(0) \text { é ponto de transição } \Longleftrightarrow \frac{k_{2}^{+}-k_{1}}{k_{3}^{+}}=\frac{k_{2}^{-}-k_{1}}{k_{3}^{-}} \quad \text { e } \quad k_{3}^{+} \cdot k_{3}^{-}<0 .
$$

Veja figura 2.9.

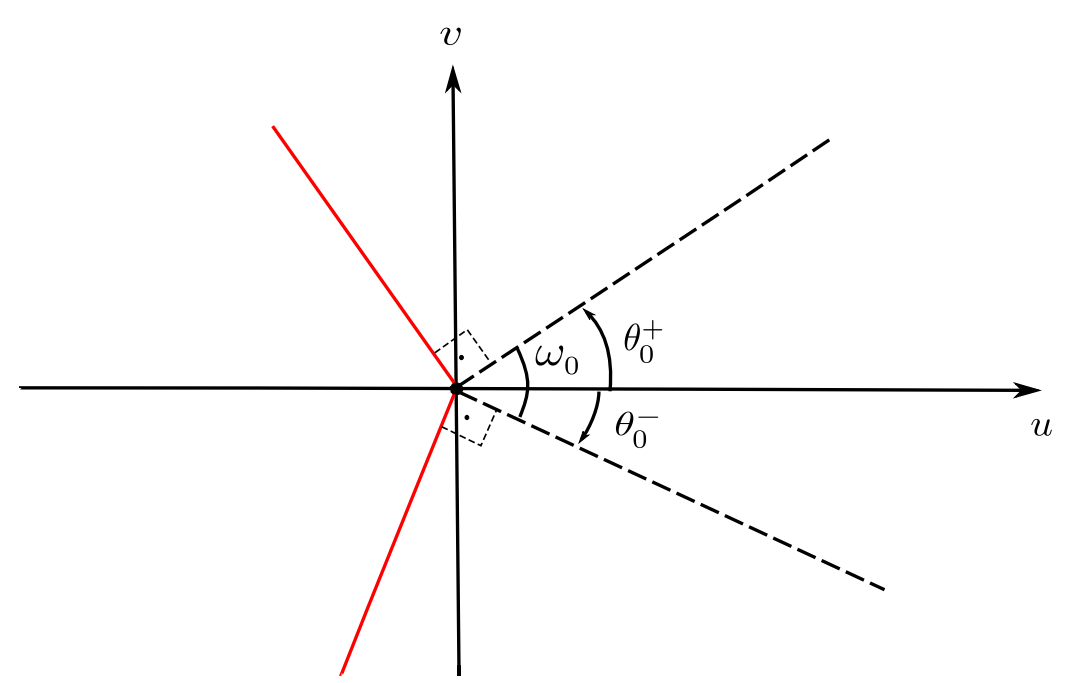

Figura 2.9: Exemplos de ângulos formados entre as linhas principais minimas (linhas tracejadas) e as linhas principais máximas (linhas contínuas) de $\mathbf{S}^{+}$e de $\mathbf{S}^{-}$com o eixo-u.

Demonstração. Para o caso em que $k_{3}^{+} \neq 0$ e $k_{3}^{-} \neq 0$ as linhas de curvatura principal de ambas as superfícies $\mathbf{S}^{+}$e $\mathbf{S}^{-}$são transversais ao bordo $\boldsymbol{\delta}$ em uma vizinhança da origem. O primeiro passo é determinar as curvaturas principais das superfícies $\mathbf{S}^{+}$e $\mathbf{S}^{-}$ em $(u, v)=(0,0)$ e, posteriormente, usando o sistema (1.3) do capítulo 1. encontrar as condições para que $\omega_{0}=\pi$.

No que segue, $k_{(1,+)} \leq k_{(2,+)}$ são as curvaturas principais da superfície $\mathbf{S}^{+}$em $(0,0)$ e $k_{(1,-)} \leq k_{(2,-)}$ são as curvaturas principais da superfície $\mathbf{S}^{-}$em $\left.(0,0)\right]^{3}$ Das expressões de

\footnotetext{
${ }^{3}$ Lembremos que, em termos dos coeficientes das formas fundamentais, as curvaturas Gaussiana $(\mathcal{K})$
} e Média $(\mathcal{H})$ são dadas respectivamente por:

$$
\mathcal{K}=k_{1} \cdot k_{2}=\frac{1}{|\mathcal{N}|^{2}} \frac{e g-f^{2}}{E G-F^{2}}, \quad \mathcal{H}=\frac{k_{1}+k_{2}}{2}=\frac{1}{|\mathcal{N}|} \frac{e G-2 f F+g E}{2\left(E G-F^{2}\right)} .
$$

onde $k_{1} \leq k_{2}$ são as curvaturas principais e $\mathcal{N}$ o vetor normal à superfície.

Portanto, $k_{1}$ e $k_{2}$ podem ser expressas por:

$$
k_{1}=\mathcal{H}-\sqrt{\mathcal{H}^{2}-\mathcal{K}}, \quad k_{2}=\mathcal{H}+\sqrt{\mathcal{H}^{2}-\mathcal{K}}
$$


$E, F, G, e, f$ e $g$ encontradas em (2.6), 2.7), 2.9) e 2.10) temos:

$$
\begin{aligned}
& k_{(1,+)}(0,0)=\frac{1}{2}\left(k_{1}+k_{2}^{+}-\sqrt{\left(k_{2}^{+}-k_{1}\right)^{2}+4\left(k_{3}^{+}\right)^{2}}\right), \\
& k_{(2,+)}(0,0)=\frac{1}{2}\left(k_{1}+k_{2}^{+}+\sqrt{\left(k_{2}^{+}-k_{1}\right)^{2}+4\left(k_{3}^{+}\right)^{2}}\right), \\
& k_{(1,-)}(0,0)=\frac{1}{2}\left(k_{1}+k_{2}^{-}-\sqrt{\left(k_{2}^{-}-k_{1}\right)^{2}+4\left(k_{3}^{-}\right)^{2}}\right), \\
& k_{(2,-)}(0,0)=\frac{1}{2}\left(k_{1}+k_{2}^{-}+\sqrt{\left(k_{2}^{-}-k_{1}\right)^{2}+4\left(k_{3}^{-}\right)^{2}}\right) .
\end{aligned}
$$

Do sistema 1.3 do capítulo 1 temos que as direções principais, relativas à superfície $\mathbf{S}^{+}(v \geq 0)$, são soluções do sistema:

$$
\left\{\begin{array}{c}
\left(\frac{e}{\left|\mathcal{N}^{+}\right|}-k_{(i,+)} E\right) d u+\left(\frac{f}{\left|\mathcal{N}^{+}\right|}-k_{(i,+)} F\right) d v=0 \\
\left(\frac{f}{\left|\mathcal{N}^{+}\right|}-k_{(i,+)} F\right) d u+\left(\frac{g}{\left|\mathcal{N}^{+}\right|}-k_{(i,+)} G\right) d v=0
\end{array}\right.
$$

onde $k_{(i,+)}(i=1,2)$ são as curvaturas principais determinadas em $(2.17)$ e $\mathcal{N}^{+}$o vetor normal à superfície $\mathbf{S}^{+}$definido em 2.5). Agora, substituindo em 2.18 as expressões de $E(0,0), F(0,0), G(0,0), e(0,0), f(0,0)$ e $g(0,0)$ dadas por 2.6) e 2.7) e observando que $\left|\mathcal{N}^{+}(0,0)\right|=1$ encontramos dois sistemas:

Sistema que determina a direção principal mínima em $\mathbf{S}^{+}$:

$$
\left\{\begin{array}{l}
\frac{1}{2}\left(k_{1}-k_{2}^{+}+\sqrt{\left(k_{2}^{+}-k_{1}\right)^{2}+4\left(k_{3}^{+}\right)^{2}}\right) d u+k_{3}^{+} d v=0 \\
k_{3}^{+} d u+\frac{1}{2}\left(k_{2}^{+}-k_{1}+\sqrt{\left(k_{2}^{+}-k_{1}\right)^{2}+4\left(k_{3}^{+}\right)^{2}}\right) d v=0
\end{array}\right.
$$

Sistema que determina a direção principal máxima em $\mathbf{S}^{+}$:

$$
\left\{\begin{array}{l}
\frac{1}{2}\left(k_{1}-k_{2}^{+}-\sqrt{\left(k_{2}^{+}-k_{1}\right)^{2}+4\left(k_{3}^{+}\right)^{2}}\right) d u+k_{3}^{+} d v=0 \\
k_{3}^{+} d u+\frac{1}{2}\left(k_{2}^{+}-k_{1}-\sqrt{\left(k_{2}^{+}-k_{1}\right)^{2}+4\left(k_{3}^{+}\right)^{2}}\right) d v=0
\end{array} .\right.
$$

Analogamente ao que foi feito para a superfície $\mathbf{S}^{+}$temos que as direções principais, 
relativas à superfície $\mathbf{S}^{-}(v \leq 0)$, são soluções do sistema:

$$
\left\{\begin{array}{l}
\left(\frac{e}{\left|\mathcal{N}^{-}\right|}-k_{(i,-)} E\right) d u+\left(\frac{f}{\left|\mathcal{N}^{-}\right|}-k_{(i,-)} F\right) d v=0 \\
\left(\frac{f}{\left|\mathcal{N}^{-}\right|}-k_{(i,-)} F\right) d u+\left(\frac{g}{\left|\mathcal{N}^{-}\right|}-k_{(i,-)} G\right) d v=0
\end{array},\right.
$$

onde $k_{(i,-)}(i=1,2)$ são as curvaturas principais determinadas em 2.17 e $\mathcal{N}^{-}$o vetor normal à superfície $\mathbf{S}^{-}$definido em (2.8). Agora, substituindo em 2.21) as expressões de $E(0,0), F(0,0), G(0,0), e(0,0), f(0,0)$ e $g(0,0)$ dadas por 2.9) e 2.10 e observando que $\left|\mathcal{N}^{-}(0,0)\right|=1$ temos dois sistemas:

Sistema que determina a direção principal mínima em $\mathbf{S}^{-}$:

$$
\left\{\begin{array}{l}
\frac{1}{2}\left(k_{1}-k_{2}^{-}+\sqrt{\left(k_{2}^{-}-k_{1}\right)^{2}+4\left(k_{3}^{-}\right)^{2}}\right) d u+k_{3}^{-} d v=0 \\
k_{3}^{-} d u+\frac{1}{2}\left(k_{2}^{-}-k_{1}+\sqrt{\left(k_{2}^{-}-k_{1}\right)^{2}+4\left(k_{3}^{-}\right)^{2}}\right) d v=0
\end{array}\right.
$$

Sistema que determina a direção principal máxima em $\mathbf{S}^{-}$:

$$
\left\{\begin{array}{l}
\frac{1}{2}\left(k_{1}-k_{2}^{-}-\sqrt{\left(k_{2}^{-}-k_{1}\right)^{2}+4\left(k_{3}^{-}\right)^{2}}\right) d u+k_{3}^{-} d v=0 \\
k_{3}^{-} d u+\frac{1}{2}\left(k_{2}^{-}-k_{1}-\sqrt{\left(k_{2}^{-}-k_{1}\right)^{2}+4\left(k_{3}^{-}\right)^{2}}\right) d v=0
\end{array} .\right.
$$

Supondo, sem perda de generalidade, que $\theta_{0}^{+}$(respectivamente $\left.\theta_{0}^{-}\right)$é o ângulo entre o eixo- $u$ positivo e a linha principal mínima da superfície $\mathbf{S}^{+}$(respectivamente $\mathbf{S}^{-}$), temos que $\tan \left(\theta_{0}^{+}\right)=d v / d u$ (respectivamente $\tan \left(\theta_{0}^{-}\right)=d v / d u$ ) é dada por qualquer uma das equações do sistema 2.19) (respectivamente (2.22) ) acima.

Observe que $\omega_{0}=\pi$ se, e somente se, $\tan \left(\theta_{0}^{+}\right) \cdot \tan \left(\theta_{0}^{-}\right)=-1$.

Da primeira equação do sistema (2.19) e do sistema 2.22 (também poderíamos ter escolhido a segunda equação destes sistemas) obtemos que

$$
\begin{aligned}
& \tan \left(\theta_{0}^{+}\right)=\left(\frac{d v}{d u}\right)^{+}=-\frac{k_{1}-k_{2}^{+}+\sqrt{\left(k_{2}^{+}-k_{1}\right)^{2}+4\left(k_{3}^{+}\right)^{2}}}{2 k_{3}^{+}} \\
& \tan \left(\theta_{0}^{-}\right)=\left(\frac{d v}{d u}\right)^{-}=-\frac{k_{1}-k_{2}^{-}+\sqrt{\left(k_{2}^{-}-k_{1}\right)^{2}+4\left(k_{3}^{-}\right)^{2}}}{2 k_{3}^{-}}
\end{aligned}
$$

Pode-se verificar que a condição (2.16) encontrada é a mesma para ambas equações dos sistemas 2.19 e 2.22 escolhidas. 
Portanto, $\tan \left(\theta_{0}^{+}\right) \cdot \tan \left(\theta_{0}^{-}\right)=-1$ se, e somente se,

$$
\left(k_{1}-k_{2}^{+}+\sqrt{\left(k_{2}^{+}-k_{1}\right)^{2}+4\left(k_{3}^{+}\right)^{2}}\right) \cdot\left(k_{1}-k_{2}^{-}+\sqrt{\left(k_{2}^{-}-k_{1}\right)^{2}+4\left(k_{3}^{-}\right)^{2}}\right)=-4 k_{3}^{+} k_{3}^{-} \text {. }
$$

A equação 2.24 é satisfeita se $\frac{k_{2}^{+}-k_{1}}{k_{3}^{+}}=\frac{k_{2}^{-}-k_{1}}{k_{3}^{-}}$e $k_{3}^{+} \cdot k_{3}^{-}<0$.

De fato, observe que

$$
\frac{k_{2}^{+}-k_{1}}{k_{3}^{+}}=\frac{k_{2}^{-}-k_{1}}{k_{3}^{-}} \Longleftrightarrow k_{3}^{-}=\frac{k_{3}^{+}\left(k_{2}^{-}-k_{1}\right)}{k_{2}^{+}-k_{1}}
$$

logo, substituindo $k_{3}^{-}=\frac{k_{3}^{+}\left(k_{2}^{-}-k_{1}\right)}{k_{2}^{+}-k_{1}}$ no lado esquerdo da equação 2.24 e considerando que $k_{3}^{+} \cdot k_{3}^{-}<0$, obtemos

$$
\begin{aligned}
& \left(k_{1}-k_{2}^{+}+\sqrt{\left(k_{2}^{+}-k_{1}\right)^{2}+4\left(k_{3}^{+}\right)^{2}}\right)\left(k_{1}-k_{2}^{-}+\sqrt{\left(k_{2}^{-}-k_{1}\right)^{2}+4\left(\frac{k_{3}^{+}\left(k_{2}^{-}-k_{1}\right)}{k_{2}^{+}-k_{1}}\right)^{2}}\right) \\
= & \left(k_{1}-k_{2}^{+}+\sqrt{\left(k_{2}^{+}-k_{1}\right)^{2}+4\left(k_{3}^{+}\right)^{2}}\right)\left(k_{1}-k_{2}^{-}+\sqrt{\frac{\left(k_{2}^{-}-k_{1}\right)^{2}\left[\left(k_{2}^{+}-k_{1}\right)^{2}+4\left(k_{3}^{+}\right)^{2}\right]}{\left(k_{2}^{+}-k_{1}\right)^{2}}}\right) \\
= & \left(k_{1}-k_{2}^{+}+\sqrt{\left(k_{2}^{+}-k_{1}\right)^{2}+4\left(k_{3}^{+}\right)^{2}}\right)\left(k_{1}-k_{2}^{-}+\left|\frac{k_{2}^{-}-k_{1}}{k_{2}^{+}-k_{1}}\right| \sqrt{\left(k_{2}^{+}-k_{1}\right)^{2}+4\left(k_{3}^{+}\right)^{2}}\right) \\
\stackrel{(*)}{=} & \left(k_{1}-k_{2}^{+}+\sqrt{\left(k_{2}^{+}-k_{1}\right)^{2}+4\left(k_{3}^{+}\right)^{2}}\right)\left(k_{1}-k_{2}^{-}-\frac{k_{2}^{-}-k_{1}}{k_{2}^{+}-k_{1}} \sqrt{\left(k_{2}^{+}-k_{1}\right)^{2}+4\left(k_{3}^{+}\right)^{2}}\right) \\
= & \frac{k_{2}^{-}-k_{1}}{k_{2}^{+}-k_{1}}\left[\left(k_{1}-k_{2}^{+}\right)+\sqrt{\left(k_{2}^{+}-k_{1}\right)^{2}+4\left(k_{3}^{+}\right)^{2}}\right]\left[\left(k_{1}-k_{2}^{+}\right)-\sqrt{\left(k_{2}^{+}-k_{1}\right)^{2}+4\left(k_{3}^{+}\right)^{2}}\right] \\
= & \frac{k_{2}^{-}-k_{1}}{k_{2}^{+}-k_{1}}\left[\left(k_{1}-k_{2}^{+}\right)^{2}-\left(k_{2}^{+}-k_{1}\right)^{2}-4\left(k_{3}^{+}\right)^{2}\right]=-4 k_{3}^{+}\left[k_{3}^{+}\left(\frac{k_{2}^{-}-k_{1}}{k_{2}^{+}-k_{1}}\right)^{2}=-4 k_{3}^{+} k_{3}^{-} .\right. \\
& (*) \mid \frac{k_{2}^{-}-k_{1}}{k_{2}^{+}-k_{1}}=-\frac{k_{2}^{-}-k_{1}}{k_{2}^{+}-k_{1}} \text { pois, por hipótese, } k_{3}^{+} \cdot k_{3}^{-}<0 \mathrm{e} k_{3}^{-}=k_{3}^{+}\left(\frac{k_{2}^{-}-k_{1}}{\left.k_{2}^{+}-k_{1}\right)^{2}} .\right.
\end{aligned}
$$

Portanto a equação (2.24) é verificada e isto completa a demonstração da proposição.

Estabelecemos na proposição 2.1.8 as condições para que $\delta(0)$ seja um ponto de tran- 
sição, no caso em que $k_{3}^{+} \cdot k_{3}^{-} \neq 0$. Vamos agora determinar as hipóteses para que $\delta(u)$ seja um ponto de costura quando $u>0$ e um ponto de deslizamento quando $u<0$ para as linhas de curvatura principal mínima (veja figura 2.10). Como acima, considere $\theta^{+}(u) \in[0, \pi / 2)$ (respectivamente $\theta^{-}(u) \in[0,-\pi / 2)$ ) o ângulo entre a linha principal mínima de $\mathbf{S}^{+}$(respectivamente $\mathbf{S}^{-}$) com o bordo $\boldsymbol{\delta}$ no ponto $\delta(u)$ e $\omega(u)=\left(\theta^{+}-\theta^{-}\right)(u)$.

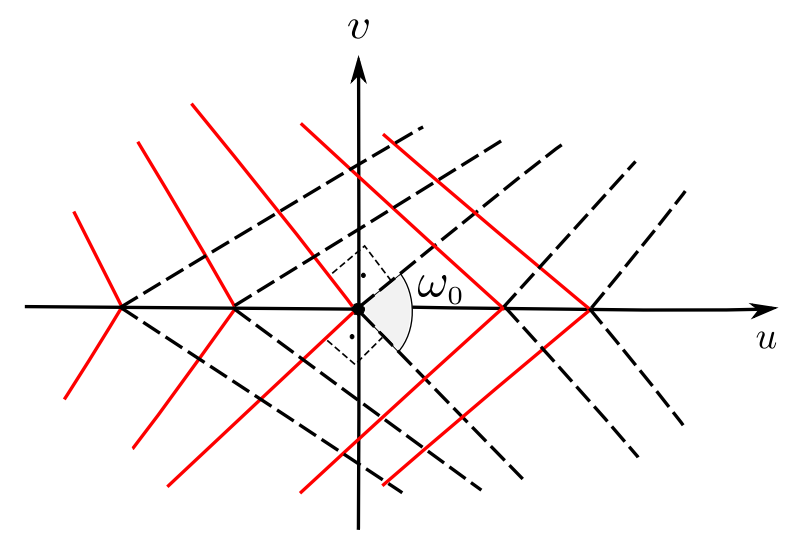

Figura 2.10: A origem $\delta(0)$ é um ponto de transição e, para as linhas de curvatura principal minima, $\delta(u)$ é ponto de costura se $u>0$ e ponto de deslizamento se $u<0$. Linhas tracejadas (respectivamente contínuas) ilustram as linhas de curvatura principal minima (respectivamente máxima).

Proposição 2.1.9. Suponha que $k_{3}^{+}<0<k_{3}^{-}, \quad k_{2}^{+}<k_{1}<k_{2}^{-}$,

$$
\frac{k_{2}^{+}-k_{1}}{k_{3}^{+}}=\frac{k_{2}^{-}-k_{1}}{k_{3}^{-}} \quad \text { e } k_{3}^{-}>\frac{\left(k_{1}-k_{2}^{-}\right)\left[\gamma\left(k_{1}-k_{2}^{-}\right)+c\left(k_{1}-k_{2}^{-}\right)\right]}{(a-b)\left(k_{1}-k_{2}^{-}\right)+(a-\beta)\left(k_{1}-k_{2}^{+}\right)} .
$$

Então, para as linhas de curvatura principal mínima, $\delta(u)$ é um ponto de costura se $u>0$ $e$ de deslizamento se $u<0$.

Demonstração. O primeiro passo é determinar as curvaturas principais das superfícies $\mathbf{S}^{+}$ e $\mathbf{S}^{-}$em $(u, v)=(u, 0)$ e, posteriormente, usando o sistema 1.3 do capítulo 1, encontrar as condições para que $\delta(u)$ seja ponto de deslizamento para $u<0$ e de costura para $u>0$.

No que segue, $k_{(1,+)} \leq k_{(2,+)}$ são as curvaturas principais da superfície $\mathbf{S}^{+}$em $(u, 0)$ e $k_{(1,-)} \leq k_{(2,-)}$ são as curvaturas principais da superfície $\mathbf{S}^{-}$em $(u, 0)$. Usando as expressões de $E, F, G, e, f, g, \mathcal{N}^{+}$e $\mathcal{N}^{-}$, respectivamente em (2.6), 2.7), 2.9), 2.10), 2.5 e 2.8) determinamos $k_{(i,+)}(u, 0)$ e $k_{(i,-)}(u, 0), i=1,2$.

Do sistema 1.3 do capítulo 1 temos que as direções principais mínimas, relativas à superfície $\mathbf{S}^{+}(v \geq 0)$, são soluções de:

$$
\left\{\begin{array}{l}
\left(\frac{e}{\left|\mathcal{N}^{+}\right|}-k_{(1,+)} E\right) d u+\left(\frac{f}{\left|\mathcal{N}^{+}\right|}-k_{(1,+)} F\right) d v=0 \\
\left(\frac{f}{\left|\mathcal{N}^{+}\right|}-k_{(1,+)} F\right) d u+\left(\frac{g}{\left|\mathcal{N}^{+}\right|}-k_{(1,+)} G\right) d v=0
\end{array},\right.
$$


onde $k_{(1,+)}(u, 0)$ é curvatura principal mínima e $\mathcal{N}^{+}(u, 0)$ o vetor normal de $\mathbf{S}^{+}$dado pela equação 2.5 .

Analogamente para a superfície $\mathbf{S}^{-}(v \leq 0)$, temos que as direções principais mínimas são soluções do sistema:

$$
\left\{\begin{array}{l}
\left(\frac{e}{\left|\mathcal{N}^{-}\right|}-k_{(1,-)} E\right) d u+\left(\frac{f}{\left|\mathcal{N}^{-}\right|}-k_{(1,-)} F\right) d v=0 \\
\left(\frac{f}{\left|\mathcal{N}^{-}\right|}-k_{(1,-)} F\right) d u+\left(\frac{g}{\left|\mathcal{N}^{-}\right|}-k_{(1,-)} G\right) d v=0
\end{array}\right.
$$

onde $k_{(1,-)}(u, 0)$ é curvatura principal mínima e $\mathcal{N}^{-}(u, 0)$ o vetor normal de $\mathbf{S}^{-}$dado pela equação (2.8).

Vamos definir a seguinte função:

$$
\chi(u):=\left[\tan \theta^{+}(u)\right]\left[\tan \theta^{-}(u)\right]=\left(\frac{d v}{d u}\right)^{+}\left(\frac{d v}{d u}\right)^{-}(u)
$$

onde $\left(\frac{d v}{d u}\right)^{ \pm}$foram obtidos dos sistemas 2.25 e 2.26 .

Substituindo $k_{3}^{+}=\frac{k_{3}^{-}\left(k_{2}^{+}-k_{1}\right)}{k_{2}^{-}-k_{1}}$ (condição para que em $u=0$ tenhamos um ponto de transição) na expressão para $\chi$ em 2.27 obtemos $\chi(u)=-1+\mathcal{M}_{1} u+\mathcal{M}_{2} u^{2}+\cdots$, onde

$$
\begin{aligned}
\mathcal{M}_{1}= & -\left\{\frac{k_{3}^{-}\left[\left(k_{1}-k_{2}^{-}\right)(b-a)+\left(k_{1}-k_{2}^{+}\right)(\beta-a)\right]}{2\left(k_{3}^{-}\right)^{3}\left(k_{1}-k_{2}^{+}\right)\left[4\left(k_{3}^{-}\right)^{2}+\left(k_{1}-k_{2}^{-}\right)^{2}\right]}+\right. \\
& \left.+\frac{\gamma\left(k_{1}-k_{2}^{+}\right)\left(k_{1}-k_{2}^{-}\right)+c\left(k_{1}-k_{2}^{-}\right)^{2}}{2\left(k_{3}^{-}\right)^{3}\left(k_{1}-k_{2}^{+}\right)\left[4\left(k_{3}^{-}\right)^{2}+\left(k_{1}-k_{2}^{-}\right)^{2}\right]}\right\} \cdot \\
& \left\{\left(k_{1}-k_{2}^{-}\right)\left[4\left(k_{3}^{-}\right)^{2}+\left(k_{1}-k_{2}^{-}\right)^{2}\right]+\sqrt{4\left(k_{3}^{-}\right)^{2}+\left(k_{1}-k_{2}^{-}\right)^{2}}\left[\left(k_{1}-k_{2}^{-}\right)^{2}+2\left(k_{3}^{-}\right)^{2}\right]\right\} .
\end{aligned}
$$

Portanto, se considerarmos $\mathcal{M}_{1}<0$, teremos:

1) $u>0 \Longrightarrow \chi(u)=-1+\mathcal{M}_{1} u+\cdots<-1 \Longrightarrow \delta(u)$ é ponto de costura;

2) $u<0 \Longrightarrow \chi(u)=-1+\mathcal{M}_{1} u+\cdots>-1 \Longrightarrow \delta(u)$ é ponto de deslizamento.

Como $k_{3}^{-}>0$ e $k_{2}^{+}<k_{1}<k_{2}^{-}$, o denominador de $\mathcal{M}_{1}$ torna-se positivo. Fazendo um 
estudo do sinal do numerador da função $\mathcal{M}_{1}$ na variável $k_{3}^{-}$concluímos o seguinte:

$$
\mathcal{M}_{1}<0 \Longleftrightarrow k_{3}^{-}>\frac{\left(k_{1}-k_{2}^{+}\right)\left[\gamma\left(k_{1}-k_{2}^{+}\right)+c\left(k_{1}-k_{2}^{-}\right)\right]}{(a-b)\left(k_{1}-k_{2}^{-}\right)+(a-\beta)\left(k_{1}-k_{2}^{+}\right)} .
$$

Isto completa a demonstração.

\subsection{3 $\quad 3^{\circ}$ Caso: Ponto de Dobra}

A última possibilidade a ser analisada é o caso em que $k_{3}^{+}=0$ e $k_{3}^{-} \neq 0$ (ou equivalentemente, $k_{3}^{+} \neq 0$ e $\left.k_{3}^{-}=0\right)$. Definimos esta situação a seguir.

Definição 2.1.10. [Ponto de Dobra] Um ponto sobre a curva bordo $\boldsymbol{\delta}$ é um ponto de $\boldsymbol{\delta}$-dobra se é um ponto de tangência quadrática das linhas de curvatura da superfície $\mathbf{S}^{+}$(respectivamente da superfície $\mathbf{S}^{-}$) com $\boldsymbol{\delta}$ e um ponto de cruzamento transversal das linhas de curvatura da superfície $\mathbf{S}^{-}$(respectivamente da superfície $\mathbf{S}^{+}$) com $\boldsymbol{\delta}$.

As figuras 2.11, abaixo, são exemplos de pontos de $\boldsymbol{\delta}$-dobras.

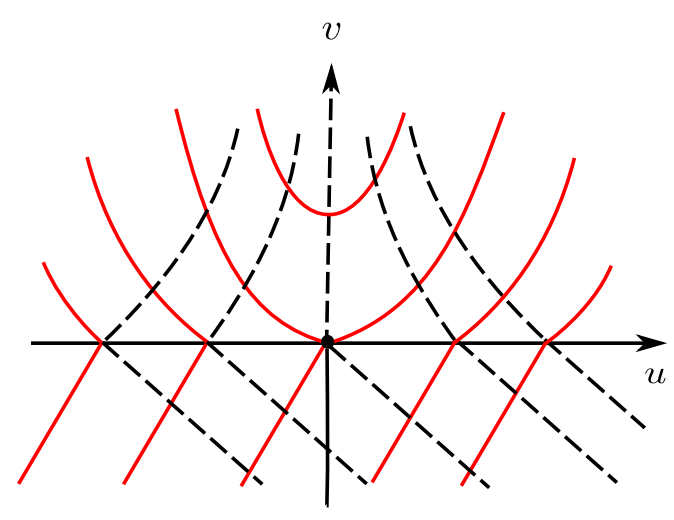

(i) $k_{3}^{+}=0, k_{3}^{-} \neq 0$ e $c>0$

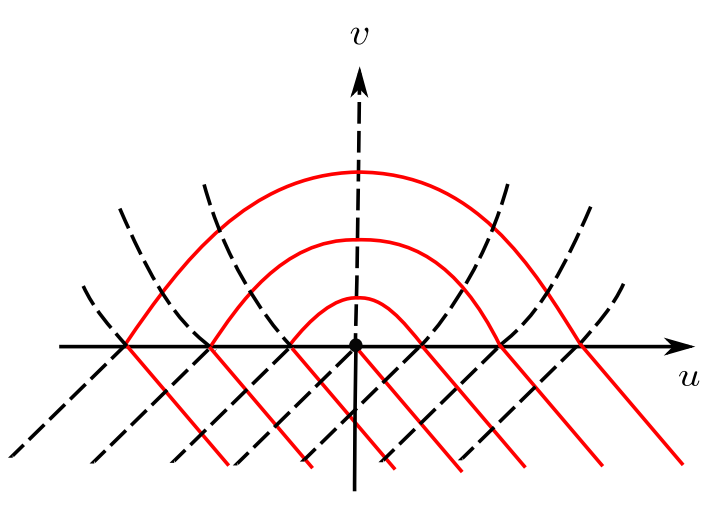

(ii) $k_{3}^{+}=0, k_{3}^{-} \neq 0$ e $c<0$

Figura 2.11: Configuração principal suave por partes de $\mathbf{S}=\mathbf{S}^{+} \cup \boldsymbol{\delta} \cup \mathbf{S}^{-}$em uma vizinhança de um ponto de $\boldsymbol{\delta}$-dobra, considerando $k_{2}^{+}<k_{1}$. Linhas tracejadas (respectivamente contínuas) ilustram as linhas de curvatura principal mínima (respectivamente máxima).

Observação 2.1.11. Para o caso em que $k_{2}^{+}>k_{1}, k_{3}^{+}=0$ e $k_{3}^{-} \neq 0$, a configuração principal suave por partes da superfície $\mathbf{S}$ é topologicamente equivalente à da figura 2.11.

\subsection{Regularização da Superfície S em uma Carta Lo- cal}

Nesta seção definiremos a regularização da superfície suave por partes $\mathbf{S}$ ao longo da curva $\boldsymbol{\delta}$ e especificaremos o tipo de função de transição usada. O resultado da regularização é uma superfície de classe $C^{\infty}$ para a qual podemos aplicar a teoria clássica. 
Definição 2.2.1. [Função de transição] Uma função $\phi: \mathbb{R} \rightarrow \mathbb{R}, \phi \in C^{\infty}$, é uma função de transição se $\phi^{\prime}(t)>0$, para $t \in(-1,1)$ e $\phi(t)=\left\{\begin{array}{l}0, t \leq-1 \\ 1, \quad t \geq 1\end{array}\right.$. Veja figura 2.12

Em [5], Garcia e Sotomayor definiram a regularização de superfícies em $\mathbb{R}^{3}$ usando uma função de transição específica. Para $\varepsilon>0$ suficientemente pequeno, a $\varepsilon$-regularização de uma superfície $\mathbf{S}$ é um mergulho de $\mathbf{S}$ em $\mathbb{R}^{3}$ que coincide com a identidade exceto em uma E-vizinhança tubular da curva $\boldsymbol{\delta}$ e cuja imagem, $\mathbf{S}_{\varepsilon}$, é uma superfície suave (observando que $\mathbf{S}$ é suave somente no complementar da curva $\boldsymbol{\delta}$ ). Para nossos propósitos, vamos considerar a seguinte definição usando cartas locais.

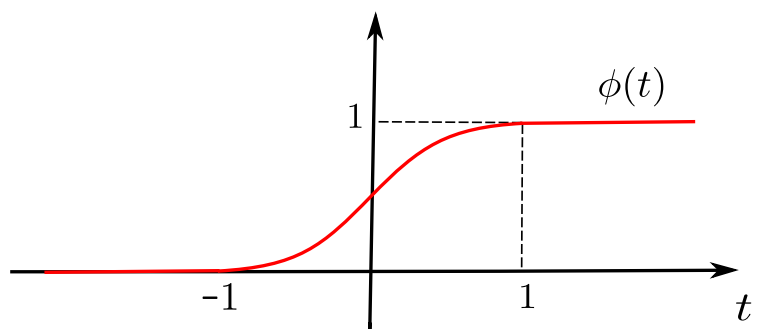

Figura 2.12: Gráfico de uma função de transição $\phi$.

Definição 2.2.2. [Regularização] Considere $\phi$ uma função de transição e $\mathbf{S}=\mathbf{S}^{+} \cup \boldsymbol{\delta} \cup \mathbf{S}^{-}$ uma superfície sem bordo em $\mathbb{R}^{3}$ contendo uma curva de descontinuidade $\boldsymbol{\delta}$, sendo $\mathbf{S}^{+}, \mathbf{S}^{-}$ e $\boldsymbol{\delta}$ definidas anteriormente. Para $\varepsilon>0$, a $\phi_{\varepsilon}$-regularização de $\mathbf{S}$, denotada por $\mathbf{S}_{\varepsilon}$, é (não unicamente) definida por uma "soma" com peso $\phi_{\varepsilon}$ das parametrizações de $\mathbf{S}^{+} e \mathbf{S}^{-}$. Mais precisamente, considerando $X^{+}(u, v)=\left(u, v, h^{+}(u, v)\right)$ e $X^{-}(u, v)=\left(u, v, h^{-}(u, v)\right)$ as parametrizações locais das superfícies $\mathbf{S}^{+}$e $\mathbf{S}^{-}$, temos que $\mathbf{S}_{\varepsilon}$ é definida por $X(u, v, \varepsilon)=$ $(u, v, h(u, v, \varepsilon))$ sendo

$$
h(u, v, \varepsilon)=\phi_{\varepsilon}(v) h^{+}(u, v)+\left[1-\phi_{\varepsilon}(v)\right] h^{-}(u, v),
$$

onde $\phi_{\varepsilon}(v)=\phi\left(\frac{v}{\varepsilon}\right) e h^{+}, h^{-}$são, respectivamente, dadas pelas equações (2.3) e 2.4) da seção 2.1, as quais serão reescritas abaixo:

$$
\begin{aligned}
& h^{+}(u, v)=\frac{1}{2} k_{1} u^{2}+k_{3}^{+} u v+\frac{1}{2} k_{2}^{+} v^{2}+\frac{1}{6} a u^{3}+\frac{1}{2} b u v^{2}+\frac{1}{2} c u^{2} v+\frac{1}{6} d v^{3}+\cdots, \quad v \geq 0, \\
& h^{-}(u, v)=\frac{1}{2} k_{1} u^{2}+k_{3}^{-} u v+\frac{1}{2} k_{2}^{-} v^{2}+\frac{1}{6} a u^{3}+\frac{1}{2} \beta u v^{2}+\frac{1}{2} \gamma u^{2} v+\frac{1}{6} \rho v^{3}+\cdots, \quad v \leq 0 .
\end{aligned}
$$

Dizemos que $\varepsilon$ é o parâmetro de regularização e para cada valor fixo de $\varepsilon>0$, a superfície $\mathbf{S}_{\varepsilon}$ é chamada de superfície regularizada. 
Denomina-se região de regularização a faixa em torno da curva $\boldsymbol{\delta}$ dada por:

$$
\delta_{\varepsilon}=\left\{\left(u, v, h^{+}(u, v)\right) \in \mathbb{R}^{3} ; v \in[0, \varepsilon)\right\} \cup\left\{\left(u, v, h^{-}(u, v)\right) \in \mathbb{R}^{3} ; v \in(-\varepsilon, 0]\right\} .
$$

É nesta região que ocorre a pró-mediação entre as superfícies $\mathbf{S}^{+}$e $\mathbf{S}^{-}$.

Observe que a função $\phi$ é estritamente crescente no intervalo $(-1,1)$ e a função $\phi_{\varepsilon}$ no intervalo $(-\varepsilon, \varepsilon)$. A figura 2.13, abaixo, é um esboço do gráfico de $\phi_{\varepsilon}(v)$ para dois valores distintos de $\varepsilon$. Para o gráfico tracejado fizemos $\varepsilon=\varepsilon_{1}$ e para o gráfico contínuo $\varepsilon=\varepsilon_{0}$, sendo $\varepsilon_{1}<\varepsilon_{0}$.

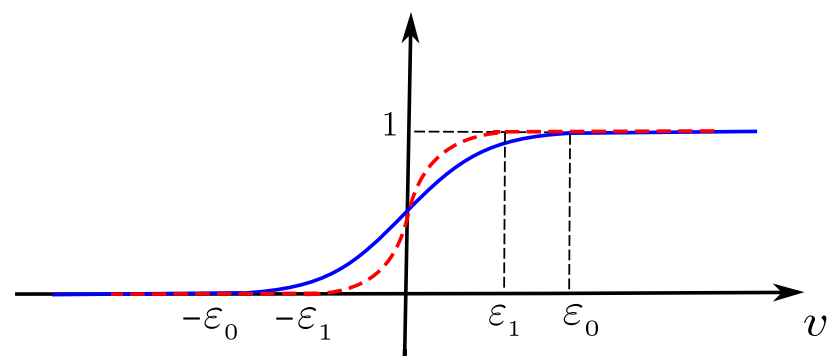

Figura 2.13: Gráficos da função de transição $\phi_{\varepsilon}$, para $\varepsilon \in\left\{\varepsilon_{0}, \varepsilon_{1}\right\}, \varepsilon_{1}<\varepsilon_{0}$.

A seguir calcularemos os coeficientes das formas fundamentais da superfície regularizada $\mathbf{S}_{\varepsilon}, \varepsilon>0$, os quais serão úteis posteriormente. Na carta $(u, v)$ temos a seguinte parametrização para $\mathbf{S}_{\varepsilon}$ :

$$
X_{\varepsilon}(u, v)=(u, v, h(u, v, \varepsilon))=\left(u, v, \phi\left(\frac{v}{\varepsilon}\right) h^{+}(u, v)+\left[1-\phi\left(\frac{v}{\varepsilon}\right)\right] h^{-}(u, v)\right) .
$$

Vamos considerar o reescalonamento $v=\varepsilon w$ na expressão (2.28). Assim, na carta $(u, w)$ a parametrização de $\mathbf{S}_{\varepsilon}$ é dada por:

$$
Y_{\varepsilon}(u, w)=(u, \varepsilon w, h(u, \varepsilon w))=\left(u, \varepsilon w, \phi(w) h^{+}(u, \varepsilon w)+[1-\phi(w)] h^{-}(u, \varepsilon w)\right) .
$$

Substituindo as expressões de $h^{+}(u, \varepsilon w)$ e $h^{-}(u, \varepsilon w)$ na equação (2.29) obtemos:

$$
\begin{aligned}
h(u, \varepsilon w) & =\frac{1}{2} k_{1} u^{2}+\varepsilon\left[k_{3}^{-}+\phi(w)\left(k_{3}^{+}-k_{3}^{-}\right)\right] u w+\frac{1}{2} \varepsilon^{2}\left[k_{2}^{-}+\phi(w)\left(k_{2}^{+}-k_{2}^{-}\right)\right] w^{2}+ \\
& +\frac{1}{6} a u^{3}+\frac{1}{2} \varepsilon[\gamma+\phi(w)(c-\gamma)] u^{2} w+\frac{1}{2} \varepsilon^{2}[\beta+\phi(w)(b-\beta)] u w^{2}+ \\
& +\frac{1}{6} \varepsilon^{3}[\rho+\phi(w)(d-\rho)] w^{3}+\frac{1}{24} A u^{4}+\frac{1}{6} \varepsilon\left[B_{1}+\phi(w)\left(B-B_{1}\right)\right] u^{3} w+ \\
& +\frac{1}{4} \varepsilon^{2}\left[C_{1}+\phi(w)\left(C-C_{1}\right)\right] w^{2} v^{2}+\frac{1}{4} \varepsilon^{3}\left[C_{1}+\phi(w)\left(C-C_{1}\right)\right] u^{2} w^{2}+ \\
& +\frac{1}{6} \varepsilon^{3}\left[D_{1}+\phi(w)\left(D-D_{1}\right)\right] u w^{3}+\frac{1}{24} \varepsilon^{4}\left[E 1-\phi(w)\left(E-E_{1}\right)\right] w^{4}+O(5) .
\end{aligned}
$$


Logo, na carta $(u, w)$ temos:

$$
\begin{aligned}
\frac{\partial h}{\partial u} & =k_{1} u+\varepsilon\left[k_{3}^{-}+\phi(w)\left(k_{3}^{+}-k_{3}^{-}\right)\right] w+\frac{1}{2} a u^{2}+\varepsilon[\gamma+\phi(w)(c-\gamma)] u w+ \\
& +\frac{1}{2} \varepsilon^{2}[\beta+\phi(w)(b-\beta)] w^{2}+\frac{1}{6} A u^{3}+\frac{1}{2} \varepsilon\left[B_{1}+\phi(w)\left(B-B_{1}\right)\right] u^{2} w+ \\
& +\frac{1}{2} \varepsilon^{2}\left[C_{1}+\phi(w)\left(C-C_{1}\right)\right] u w^{2}+\frac{1}{6} \varepsilon^{3}\left[D_{1}+\phi(w)\left(D-D_{1}\right)\right] w^{3}+O(4) \\
\frac{\partial h}{\partial w} & =\varepsilon\left[k_{3}^{-}+\phi(w)\left(k_{3}^{+}-k_{3}^{-}\right)\right] u+\varepsilon^{2}\left[k_{2}^{-}+\phi(w)\left(k_{2}^{+}-k_{2}^{-}\right)\right] w+\frac{1}{2} \varepsilon[\gamma+ \\
& +\phi(w)(c-\gamma)] u^{2}+\varepsilon\left[\phi^{\prime}(w)\left(k_{3}^{+}-k_{3}^{-}\right)+\varepsilon(\beta+\phi(w)(b-\beta))\right] u w+ \\
& +\frac{1}{2} \varepsilon^{2}\left[\phi^{\prime}(w)\left(k_{2}^{+}-k_{2}^{-}\right)+\varepsilon(\rho+\phi(w)(d-\rho))\right] w^{2}+\frac{1}{6} \varepsilon\left[B_{1}+\phi(w)\left(B-B_{1}\right)\right] u^{3}+ \\
& +\frac{1}{2} \varepsilon\left[\phi^{\prime}(w)(c-\gamma)+\varepsilon\left(C_{1}+\phi(w)\left(C-C_{1}\right)\right)\right] u^{2} w+\frac{1}{2} \varepsilon^{2}\left[\phi^{\prime}(w)(b-\beta)+\right. \\
& \left.+\varepsilon\left(D_{1}+\phi(w)\left(D-D_{1}\right)\right)\right] u w^{2}+\frac{1}{6} \varepsilon^{3}\left[\phi^{\prime}(w)(d-\rho)+\varepsilon\left(E_{1}+\phi(w)\left(E-E_{1}\right)\right)\right] w^{3}+ \\
& +\frac{1}{6} \varepsilon \phi^{\prime}(w)\left(B-B_{1}\right) u^{3} w+\frac{1}{4} \varepsilon^{2} \phi^{\prime}(w)\left(C-C_{1}\right) u^{2} w^{2}+\frac{1}{6} \varepsilon^{3} \phi^{\prime}(w)\left(D-D_{1}\right) u w^{3}+ \\
& +\frac{1}{24} \varepsilon^{4} \phi^{\prime}(w)\left(E-E_{1}\right) w^{4}+O(5) .
\end{aligned}
$$

O vetor normal (não unitário) a $\mathbf{S}_{\varepsilon}$ é dado por:

$$
\mathcal{N}_{\varepsilon}(u, w)=\left(\left(Y_{\varepsilon}\right)_{u} \wedge\left(Y_{\varepsilon}\right)_{w}\right)(u, w)=\left(1,0, \frac{\partial h}{\partial u}\right) \wedge\left(0, \varepsilon, \frac{\partial h}{\partial w}\right)=\left(-\varepsilon \frac{\partial h}{\partial u},-\frac{\partial h}{\partial w}, \varepsilon\right)
$$

sendo $\frac{\partial h}{\partial u}$ e $\frac{\partial h}{\partial w}$ obtidos em 2.30 .

Os coeficientes da Primeira Forma Fundamental de $Y_{\varepsilon}$, expressos na carta $(u, w)$, são:

$$
\begin{aligned}
E(u, w, \varepsilon) & =\left\langle\frac{\partial Y_{\varepsilon}}{\partial u}, \frac{\partial Y_{\varepsilon}}{\partial u}\right\rangle=\left\langle\left(1,0, \frac{\partial h}{\partial u}\right),\left(1,0, \frac{\partial h}{\partial u}\right)\right\rangle=1+k_{1}^{2} u^{2}+2 \varepsilon k_{1}\left[k_{3}^{-}+\right. \\
& \left.+\phi(w)\left(k_{3}^{+}-k_{3}^{-}\right)\right] u w+\varepsilon^{2}\left[k_{3}^{-}+\phi(w)\left(k_{3}^{+}-k_{3}^{-}\right)\right]^{2} w^{2}+O(3),
\end{aligned}
$$




$$
\begin{aligned}
F(u, w, \varepsilon) & =\left\langle\frac{\partial Y_{\varepsilon}}{\partial u}, \frac{\partial Y_{\varepsilon}}{\partial w}\right\rangle=\left\langle\left(1,0, \frac{\partial h}{\partial u}\right),\left(0, \varepsilon, \frac{\partial h}{\partial w}\right)\right\rangle=k_{1} \varepsilon\left[\phi(w)\left(k_{3}^{+}-k_{3}^{-}\right)+\right. \\
& \left.+k_{3}^{-}\right] u^{2}+\varepsilon^{2}\left\{\left(k_{3}^{-}\right)^{2}+k_{1} k_{2}^{-}+\phi(w)\left[k_{1}\left(k_{2}^{+}-k_{2}^{-}\right)+2 k_{3}^{-}\left(k_{3}^{+}-k_{3}^{-}\right)\right]+\right. \\
& \left.+\left[\phi(w)\left(k_{3}^{+}-k_{3}^{-}\right)\right]^{2}\right\} u w+\varepsilon^{3}\left\{k_{3}^{-} k_{2}^{-}+\phi(w)\left[k_{3}^{-}\left(k_{2}^{+}-k_{2}^{-}\right)+\right.\right. \\
& \left.\left.\left.+k_{2}^{-}\left(k_{3}^{+}-k_{3}^{-}\right)\right]+\phi(w)^{2}\left(k_{3}^{+}-k_{3}^{-}\right)\left(k_{2}^{+}-k_{2}^{-}\right)\right]\right\} w^{2}+O(3) \\
G(u, w, \varepsilon) & =\left\langle\frac{\partial Y_{\varepsilon}}{\partial w}, \frac{\partial Y_{\varepsilon}}{\partial w}\right\rangle=\left\langle\left(0, \varepsilon, \frac{\partial h}{\partial w}\right),\left(0, \varepsilon, \frac{\partial h}{\partial w}\right)\right\rangle=\varepsilon^{2}+\varepsilon^{2}\left[\phi(w)\left(k_{3}^{+}-k_{3}^{-}\right)\right. \\
& \left.+k_{3}^{-}\right]^{2} u^{2}+2 \varepsilon^{3}\left\{k_{3}^{-} k_{2}^{-}+\phi(w)\left[k_{3}^{-}\left(k_{2}^{+}-2 k_{2}^{-}\right)+k_{2}^{-} k_{3}^{+}\right]+\right. \\
& \left.+\phi(w)^{2}\left(k_{3}^{+}-k_{3}^{-}\right)\left(k_{2}^{+}-k_{2}^{-}\right)\right\} u w+\varepsilon^{4}\left[\left(k_{2}^{-}\right)^{2}+2 \phi(w) k_{2}^{-}\left(k_{2}^{+}-k_{2}^{-}\right)+\right. \\
& \left.+\phi(w)^{2}\left(k_{2}^{+}-k_{2}^{-}\right)^{2}\right] w^{2}+O(3) .
\end{aligned}
$$

Os coeficientes da Segunda Forma Fundamental de $Y_{\varepsilon}$, expressos na carta $(u, w)$, são dados por $\frac{e}{\left|\mathcal{N}_{\varepsilon}\right|}, \frac{f}{\left|\mathcal{N}_{\varepsilon}\right|}$ e $\frac{g}{\left|\mathcal{N}_{\varepsilon}\right|}$, onde $\mathcal{N}_{\varepsilon}$ é o vetor normal definido em 2.31 e

$$
\begin{aligned}
e(u, w, \varepsilon) & =\left\langle\frac{\partial^{2} Y_{\varepsilon}}{\partial u^{2}}, \mathcal{N}_{\varepsilon}\right\rangle(u, w, \varepsilon)=\varepsilon k_{1}+\varepsilon a u+\varepsilon^{2}[\gamma+\phi(w)(c-\gamma)] w+\frac{1}{2} \varepsilon A u^{2}+ \\
& +\varepsilon^{2}\left[B_{1}+\phi(w)\left(B-B_{1}\right)\right] u w+\frac{1}{2} \varepsilon^{3}\left[C_{1}+\phi(w)\left(C-C_{1}\right)\right] w^{2}+O(3) \\
f(u, w, \varepsilon) & =\left\langle\frac{\partial^{2} Y_{\varepsilon}}{\partial u \partial w}, \mathcal{N}_{\varepsilon}\right\rangle(u, w, \varepsilon)=\varepsilon^{2}\left[k_{3}^{-}+\phi(w)\left(k_{3}^{+}-k_{3}^{-}\right)\right]+\varepsilon^{2}[\phi(w)(c-\gamma)+ \\
& +\gamma] u+\varepsilon^{2}\left\{\phi^{\prime}(w)\left(k_{3}^{+}-k_{3}^{-}\right)+\varepsilon[\beta+\phi(w)(b-\beta)]\right\} w+ \\
& +\frac{1}{2} \varepsilon^{2}\left[B_{1}+\phi(w)\left(B-B_{1}\right)\right] u^{2}+\varepsilon^{2}\left\{\phi^{\prime}(w)(c-\gamma)+\varepsilon\left[\phi(w)\left(C-C_{1}\right)+\right.\right. \\
& \left.\left.+C_{1}\right]\right\} u w+\frac{1}{2} \varepsilon^{3}\left\{\phi^{\prime}(w)(b-\beta)+\varepsilon\left[D_{1}+\phi(w)\left(D-D_{1}\right)\right]\right\} w^{2}+O(3)
\end{aligned}
$$




$$
\begin{aligned}
g(u, w, \varepsilon) & =\left\langle\frac{\partial^{2} Y_{\varepsilon}}{\partial w^{2}}, \mathcal{N}_{\varepsilon}\right\rangle(u, w, \varepsilon)=\varepsilon^{3}\left[k_{2}^{-}+\phi(w)\left(k_{2}^{+}-k_{2}^{-}\right)\right]+\varepsilon^{2}\left\{2 \phi^{\prime}(w)\left(k_{3}^{+}-k_{3}^{-}\right)+\right. \\
& +\varepsilon[\beta+\phi(w)(b-\beta)]\} u+\varepsilon^{3}\left\{2 \phi^{\prime}(w)\left(k_{2}^{+}-k_{2}^{-}\right)+\varepsilon[\rho+\phi(w)(d-\rho)]\right\} w+ \\
& +\frac{1}{2} \varepsilon^{2}\left\{2 \phi^{\prime}(w)(c-\gamma)+\varepsilon\left[C_{1}+\phi(w)\left(C-C_{1}\right)\right]\right\} u^{2}+\varepsilon^{2}\left\{\phi^{\prime \prime}(w)\left(k_{3}^{+}-k_{3}^{-}\right)+\right. \\
& \left.+2 \varepsilon \phi^{\prime}(w)(b-\beta)+\varepsilon^{2}\left[D_{1}+\phi(w)\left(D-D_{1}\right)\right]\right\} u w+\frac{1}{2} \varepsilon^{3}\left\{\phi^{\prime \prime}(w)\left(k_{2}^{+}-k_{2}^{-}\right)+\right. \\
& \left.+2 \varepsilon \phi^{\prime}(w)(d-\rho)+\varepsilon^{2}\left[E_{1}+\phi(w)\left(E-E_{1}\right)\right]\right\} w^{2}+O(3) .
\end{aligned}
$$

Lembremos que, na carta $(u, w)$, a equação diferencial das linhas de curvatura é dada por:

$$
L(u, w, \varepsilon) d w^{2}+M(u, w, \varepsilon) d u d w+N(u, w, \varepsilon) d u^{2}=0,
$$

sendo $L=F g-G f, M=E g-G e$ e $N=E f-F e$.

Substituindo as expressões de $E, F$ e $G$ obtidas em (2.32) e de $e, f$ e $g$ obtidas em (2.33) temos:

$$
\begin{aligned}
L(u, w, \varepsilon) & =-\varepsilon^{4}\left\{k_{3}^{-}+\phi(w)\left(k_{3}^{+}-k_{3}^{-}\right)+[\gamma+\phi(w)(c-\gamma)] u+\right. \\
& \left.+\left[\varepsilon(\beta+\phi(w)(b-\beta))+\phi^{\prime}(w)\left(k_{3}^{+}-k_{3}^{-}\right)\right] w\right\}+O(2), \\
M(u, w, \varepsilon) & =\varepsilon^{2}\left\{\varepsilon\left[k_{2}^{-}-k_{1}+\phi(w)\left(k_{2}^{+}-k_{2}^{-}\right)\right]+[\varepsilon(\beta-a+\phi(w)(b-\beta))+\right. \\
& \left.+2 \phi^{\prime}(w)\left(k_{3}^{+}-k_{3}^{-}\right)\right] u+\varepsilon[\varepsilon \rho-\varepsilon \gamma+\varepsilon \phi(w)(\gamma-c+d-\rho)+ \\
& \left.\left.+2 \phi^{\prime}(w)\left(k_{2}^{+}-k_{2}^{-}\right)\right] w\right\}+O(2), \\
N(u, w, \varepsilon) & =\varepsilon^{2}\left\{k_{3}^{-}+\phi(w)\left(k_{3}^{+}-k_{3}^{-}\right)+[\gamma+\phi(w)(c-\gamma)] u+\right. \\
& \left.+\left[\varepsilon(\beta+\phi(w)(b-\beta))+\phi^{\prime}(w)\left(k_{3}^{+}-k_{3}^{-}\right)\right] w\right\}+O(2) .
\end{aligned}
$$

O objetivo é analisar os efeitos da regularização sobre os pontos da curva bordo comum $\boldsymbol{\delta}$ de tangência quadrática, de dobra e/ou de cruzamento transversal das linhas de curvatura de $\mathbf{S}^{+}$e $\mathbf{S}^{-}$com $\boldsymbol{\delta}$. Vamos estudar os efeitos dessa $\varepsilon$-regularização para esses três casos que foram apresentados respectivamente nas seções 2.1.1, 2.1.3 e 2.1.2. Veremos que, sob certas condições (estabelecidas nos lemas 2.2.3 e 2.2.8 e teoremas 2.2.6, 2.2.10 e 2.2 .11 de tais pontos de $\boldsymbol{\delta}$ emergem pontos umbílicos Darbouxianos sobre $\mathbf{S}_{\varepsilon}$ dos tipos $D_{1}$ e $D_{3}$, para $\varepsilon>0$ suficientemente pequeno. Nas próximas três seções apresentaremos os principais resultados obtidos para os casos citados. 


\subsection{1 $1^{\circ}$ Caso: Regularização na Vizinhança de uma Singulari- dade Quadrática $\left(k_{3}^{+}=k_{3}^{-}=0\right)$}

Nesta seção estabeleceremos as hipóteses para que da origem, $\delta(0)$, de contato quadrático das linhas de curvatura principal das superfícies $\mathbf{S}^{+}$e $\mathbf{S}^{-}$com o bordo comum $\boldsymbol{\delta}$ emerjam pontos umbílicos Darbouxianos dos tipos $D_{1}$ e $D_{3}$ sobre $\mathbf{S}_{\varepsilon}$, para $\varepsilon>0$ suficientemente pequeno. O principal resultado está contido no teorema 2.2.6.

Sabemos das proposições 2.1.1 e 2.1.3 da seção 2.1.1 que as linhas de curvatura principal das superfícies $\mathbf{S}^{+}$e $\mathbf{S}^{-}$têm contato quadrático com a curva bordo $\boldsymbol{\delta}$ na origem $\delta(0)$ se, e somente se, $k_{3}^{+}=k_{3}^{-}=0$ e $\gamma \cdot c \neq 0$.

O lema 2.2.3. seguinte, apresenta condições relativas à função de transição $\phi$ para que a origem seja um ponto umbílico da superfície regularizada $\mathbf{S}_{\varepsilon}$, para $\varepsilon>0$ suficientemente pequeno.

Lema 2.2.3. Suponha que a origem seja um ponto de tangência quadrática tal que $k_{3}^{+}=k_{3}^{-}=0, \gamma \cdot c \neq 0$, $\phi$ uma função de transição e $\mathbf{S}_{\varepsilon}$ a superfície regularizada parametrizada (como na definição 2.2.2) por $X(u, v, \varepsilon)=(u, v, h(u, v, \varepsilon)$ ), sendo

$$
h(u, v, \varepsilon)=\phi\left(\frac{v}{\varepsilon}\right) h^{+}(u, v)+\left[1-\phi\left(\frac{v}{\varepsilon}\right)\right] h^{-}(u, v) .
$$

Se $k_{2}^{+}<k_{1}<k_{2}^{-} \quad$ e $\phi(0)=\frac{k_{2}^{-}-k_{1}}{k_{2}^{-}-k_{2}^{+}} \quad$ então o conjunto

$$
U_{\varepsilon}=\{(u, v, \varepsilon): L(u, v, \varepsilon)=M(u, v, \varepsilon)=0\}
$$

é uma curva regular tal que $X\left(U_{\varepsilon}\right)=\{(0,0,0)\}$ é ponto umbílico de $\mathbf{S}_{\varepsilon}$.

Demonstração. Considerando $(u, w)=(0,0), k_{3}^{+}=k_{3}^{-}=0$ e $\phi(0)=\frac{k_{2}^{-}-k_{1}}{k_{2}^{-}-k_{2}^{+}}$nas expressões de $L(u, w)$ e $M(u, w)$ obtidas em (2.34), na carta $(u, w)$, obtemos:

$$
L(0,0, \varepsilon)=M(0,0, \varepsilon)=0, \quad \forall \varepsilon>0 .
$$

Portanto, em $(u, w)=(0,0)$ temos um ponto umbílico. Retornando às coordenadas originais $(u, v, \varepsilon)$, lembrando que $v=\varepsilon w, \varepsilon>0$ suficientemente pequeno, segue que $w=\varepsilon^{-1} v$ e a curva de pontos umbílicos é dada por $U(\varepsilon)=\{(0,0, \varepsilon), \varepsilon>0\}$.

Isto completa a demonstração.

Observação 2.2.4. A hipótese $k_{2}^{+}<k_{1}<k_{2}^{-}$no lema 2.2.3 implica

$$
0<\frac{k_{2}^{-}-k_{1}}{k_{2}^{-}-k_{2}^{+}}<1
$$

$\operatorname{logo} \phi(0) \in(0,1)$ (veja definição de $\phi$ em 2.2.1). 
Observação 2.2.5. Para o caso em que $k_{1}<k_{2}^{+}$e $k_{1}<k_{2}^{-}$a superfície regularizada $\mathbf{S}_{\varepsilon}$ não possui pontos umbílicos em uma vizinhança da origem (veja figuras 2.4. 2.14 e.2.15). De fato, vimos no lema 2.2 .3 que:

$$
M(0,0, \varepsilon)=0 \Longrightarrow \phi(0)=\phi_{\varepsilon}(0)=\frac{k_{2}^{-}-k_{1}}{k_{2}^{-}-k_{2}^{+}}, \quad \varepsilon>0 .
$$

Logo, supondo $k_{1}<k_{2}^{+}$e $k_{1}<k_{2}^{-}$, segue que $\phi(0) \notin(0,1)$, a função $M$ não se aluna em $(0,0)$ e, por continuidade de $M$, concluímos que a superfície regularizada $\mathbf{S}_{\varepsilon}$ não possui pontos umbílicos em uma vizinhança da origem, para todo $\varepsilon>0$.

As figuras 2.14 e 2.15, abaixo, representam os pontos de tangências quadráticas da figura 2.4 (à esquerda), e suas $\varepsilon$-regularizações (à direita). A região de regularização foi feita em grande escala para facilitar a visualização das linhas de curvatura principal da superfície regularizada, $\mathbf{S}_{\varepsilon}$, na vizinhança da origem.

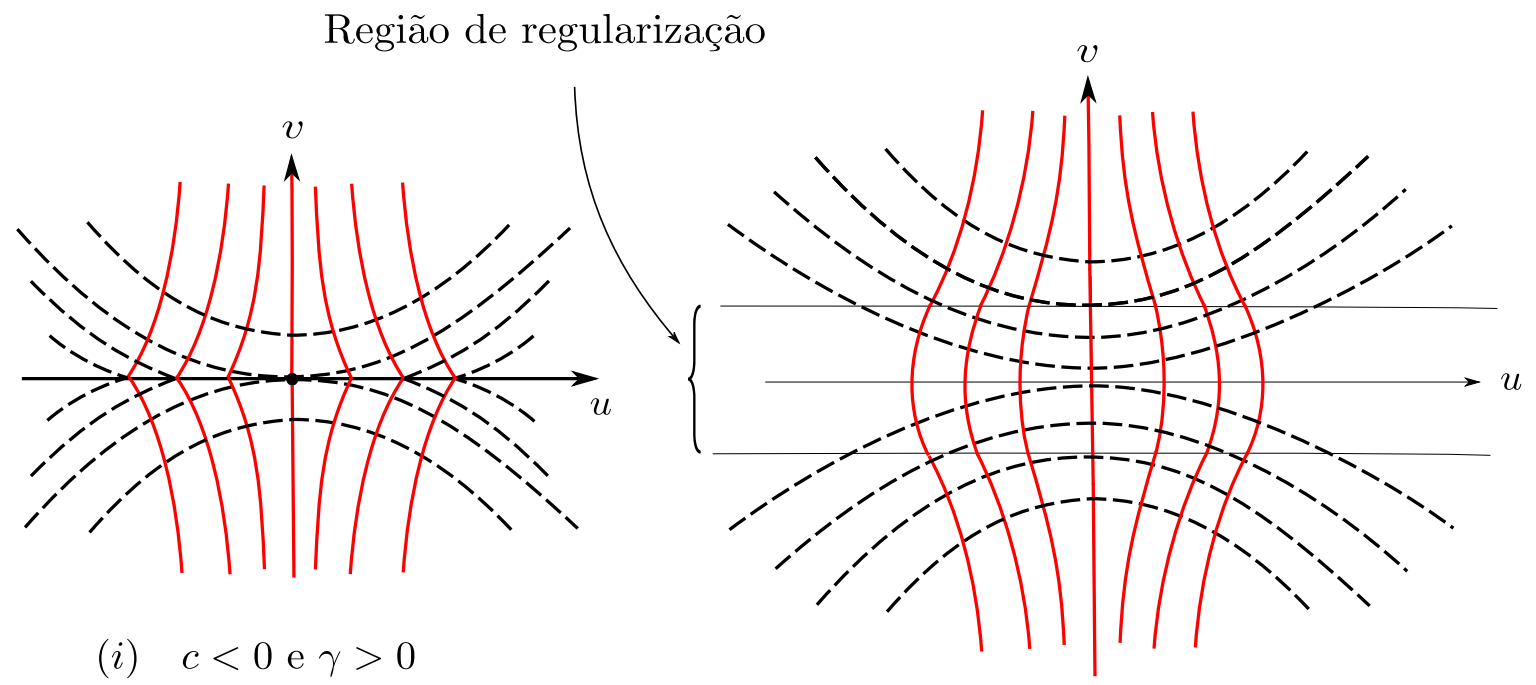

Figura 2.14: $\grave{A}$ esquerda, temos que a origem é um ponto de tangência quadrática tal que $k_{1}<k_{2}^{+}, k_{1}<k_{2}^{-}, c<0$ e $\gamma>0$ e, à direita, sua $\varepsilon$-regularização. Linhas principais mínimas (linhas tracejadas) são deslizantes e as linhas principais máximas (linhas contínuas) costuram. 


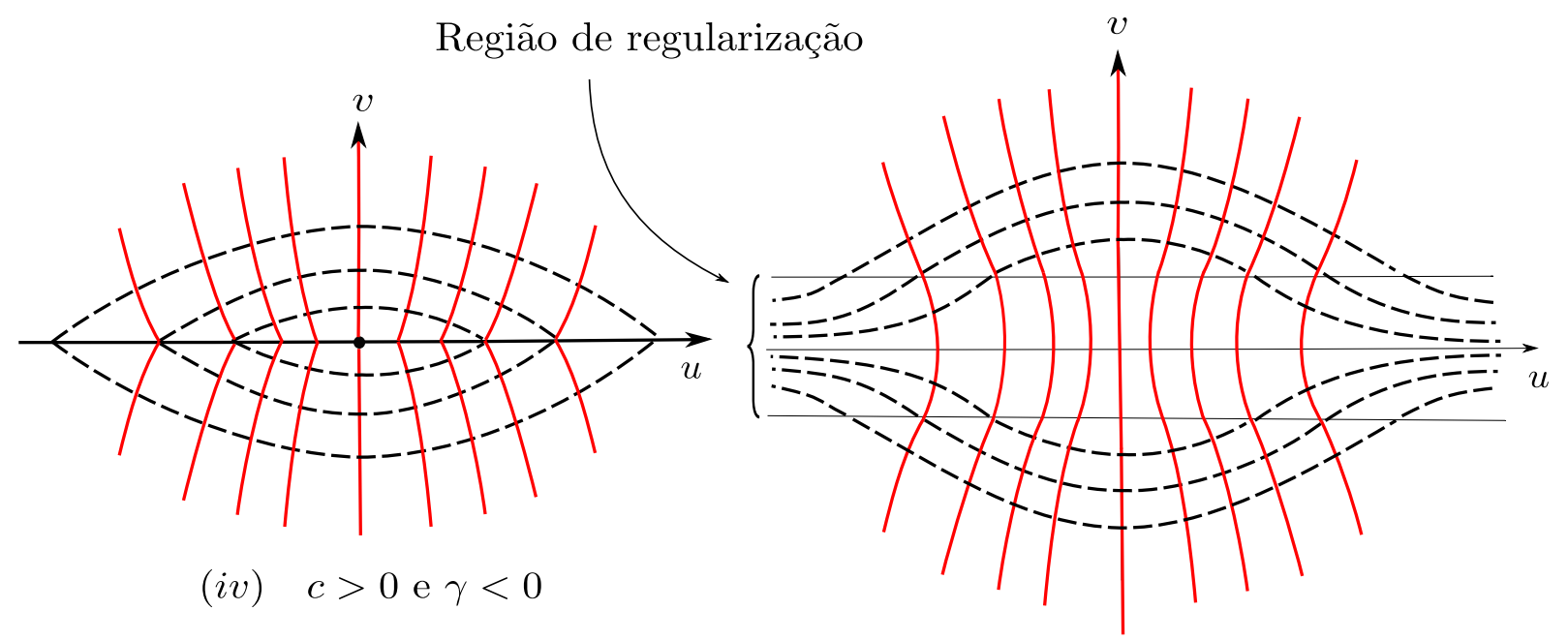

Figura 2.15: $\grave{A}$ esquerda, temos que a origem é um ponto de tangência quadrática tal que $k_{1}<k_{2}^{+}, k_{1}<k_{2}^{-}, c>0$ e $\gamma<0$ e, à direita, sua $\varepsilon$-regularização. Linhas principais mínimas (linhas tracejadas) são deslizantes e as linhas principais máximas (linhas contínuas) costuram.

O principal resultado obtido nesta seção é o próximo teorema que classifica o ponto umbílico do lema 2.2.3.

Teorema 2.2.6. Considere a superfície $\mathbf{S}=\mathbf{S}^{+} \cup \boldsymbol{\delta} \cup \mathbf{S}^{-}$e sua $\varepsilon$-regularização $\mathbf{S}_{\varepsilon}, \varepsilon>0$ suficientemente pequeno. Suponha que a origem $\delta(0)$ seja um ponto de tangência quadrática tal que $k_{3}^{+}=k_{3}^{-}=0, \gamma c \neq 0, k_{2}^{+}<k_{1}<k_{2}^{-}$e $\phi(0)=\frac{k_{2}^{-}-k_{1}}{k_{2}^{-}-k_{2}^{+}}$. Então, sobre $\mathbf{S}_{\varepsilon}$ a origem será um ponto umbílico Darbouxiano dos tipos $D_{1}$ ou $D_{3}$, sendo do tipo $D_{1}$ se $\gamma<c\left(\frac{k_{1}-k_{2}^{-}}{k_{1}-k_{2}^{+}}\right)$e do tipo $D_{3}$ se $\gamma>c\left(\frac{k_{1}-k_{2}^{-}}{k_{1}-k_{2}^{+}}\right)$.

Demonstração. Os pontos umbílicos da superfície regularizada $\mathbf{S}_{\varepsilon}$ são dados pelas equações $L(u, w, \varepsilon)=M(u, w, \varepsilon)=N(u, w, \varepsilon)=0$.

Pelo lema 2.2.3, considerando $k_{3}^{+}=k_{3}^{-}=0$ e $\phi(0)=\frac{k_{2}^{-}-k_{1}}{k_{2}^{-}-k_{2}^{+}}$, na vizinhança do ponto umbílico $(0,0)$, as expressões 2.34 de $L, M$ e $N$ tornam-se:

$$
\begin{aligned}
L(u, w, \varepsilon) & =\varepsilon^{4}\left[\frac{c\left(k_{2}^{-}-k_{1}\right)-\gamma\left(k_{2}^{+}-k_{1}\right)}{k_{2}^{+}-k_{2}^{-}}\right] u+ \\
& +\varepsilon^{5}\left[\frac{b\left(k_{2}^{-}-k_{1}\right)-\beta\left(k_{2}^{+}-k_{1}\right)}{k_{2}^{+}-k_{2}^{-}}\right] w+O(2),
\end{aligned}
$$




$$
\begin{aligned}
M(u, w, \varepsilon) & =\varepsilon^{3}\left[\frac{\beta\left(k_{2}^{+}-k_{1}\right)-a\left(k_{2}^{+}-k_{2}^{-}\right)-b\left(k_{2}^{-}-k_{1}\right)}{k_{2}^{+}-k_{2}^{-}}\right] u+ \\
& +\varepsilon^{3}\left\{\varepsilon\left[\frac{(c-d)\left(k_{2}^{-}-k_{1}\right)+(\rho-\gamma)\left(k_{2}^{+}-k_{1}\right)}{k_{2}^{+}-k_{2}^{-}}\right]+\right. \\
& \left.+3 \phi^{\prime}(0)\left(k_{2}^{+}-k_{2}^{-}\right)\right\} w+O(2), \\
N(u, w, \varepsilon) & =\varepsilon^{2}\left[\frac{\gamma\left(k_{2}^{+}-k_{1}\right)-c\left(k_{2}^{-}-k_{1}\right)}{k_{2}^{+}-k_{2}^{-}}\right] u+ \\
& +\varepsilon^{3}\left[\frac{\beta\left(k_{2}^{+}-k_{1}\right)-b\left(k_{2}^{-}-k_{1}\right)}{k_{2}^{+}-k_{2}^{-}}\right] w+O(2) .
\end{aligned}
$$

A Condição de Transversalidade T: Vamos calcular o determinante Jacobiano das funções $L(u, w, \varepsilon)$ e $M(u, w, \varepsilon)$ em $(u, w)=(0,0)$, usando as expressões de $L$ e $M$ definidas em (2.35).

$$
\operatorname{det}\left(\left.\frac{\partial(L, M)}{\partial(u, w)}\right|_{u=w=0}\right)=3 \phi^{\prime}(0)\left(k_{2}^{+}-k_{2}^{-}\right)^{2}\left[\gamma\left(k_{1}-k_{2}^{+}\right)-c\left(k_{1}-k_{2}^{-}\right)\right] \varepsilon^{7}+O(\varepsilon, 8) \text {. }
$$

Portanto, para $\varepsilon>0$ suficientemente pequeno, como $\phi^{\prime}(0)>0$ pela definição da função $\phi$, temos

$$
\operatorname{det}\left(\left.\frac{\partial(L, M)}{\partial(u, w)}\right|_{u=w=0}\right) \neq 0 \Longleftrightarrow \gamma\left(k_{1}-k_{2}^{+}\right)-c\left(k_{1}-k_{2}^{-}\right) \neq 0 \Longleftrightarrow \gamma \neq c\left(\frac{k_{1}-k_{2}^{-}}{k_{1}-k_{2}^{+}}\right) .
$$

A Condição do Discriminante D: Para $(u, w)$ próximo ao ponto umbílico $(0,0)$, a equação diferencial das linhas de curvatura da superfície $\mathbf{S}_{\varepsilon}$ é dada por:

$$
L(u, w, \varepsilon) d w^{2}+M(u, w, \varepsilon) d u d w+N(u, w, \varepsilon) d u^{2}=0,
$$

sendo as expressões de $L, M$ e $N$ dadas em 2.35 .

Vamos escrever a equação 2.37 nas coordenadas originais $(u, v, \varepsilon)$, lembrando que $v=\varepsilon w, \varepsilon>0$ suficientemente pequeno. Logo, ao substituirmos as expressões $w=v / \varepsilon$ e $d w=d v / \varepsilon$ na equação (2.37) obtemos, na carta $(u, v)$, a seguinte equação diferencial para as linhas de curvatura de $\mathbf{S}_{\varepsilon}$ :

$$
L(u, v, \varepsilon) d v^{2}+M(u, v, \varepsilon) d u d v+N(u, v, \varepsilon) d u^{2}=0
$$


sendo

$$
\begin{aligned}
L(u, v, \varepsilon) & =\varepsilon\left[\gamma\left(k_{1}-k_{2}^{+}\right)+c\left(k_{2}^{-}-k_{1}\right)\right] u+ \\
& +\varepsilon\left[\beta\left(k_{1}-k_{2}^{+}\right)+b\left(k_{2}^{-}-k_{1}\right)\right] v+O(2) \\
M(u, v, \varepsilon) & =\varepsilon\left[a\left(k_{2}^{-}-k_{2}^{+}\right)+b\left(k_{1}-k_{2}^{-}\right)+\beta\left(k_{2}^{+}-k_{1}\right)\right] u+ \\
& +\left\{\varepsilon\left[(d-c)\left(k_{1}-k_{2}^{-}\right)+(\gamma-\rho)\left(k_{1}-k_{2}^{+}\right)\right]+\right. \\
& \left.+3 \phi^{\prime}(0)\left(k_{2}^{+}-k_{2}^{-}\right)^{2}\right\} v+O(2), \\
N(u, v, \varepsilon) & =-\varepsilon\left[\gamma\left(k_{1}-k_{2}^{+}\right)+c\left(k_{2}^{-}-k_{1}\right)\right] u- \\
& -\varepsilon\left[\beta\left(k_{1}-k_{2}^{+}\right)+b\left(k_{2}^{-}-k_{1}\right)\right] v+O(2) .
\end{aligned}
$$

Na carta $(u, v ; q)$, sendo $q=d u / d v$, vamos considerar a superfície de Lie-Cartan $\mathcal{G}^{-1}\{0\}$ sobre a qual temos definido o campo de Lie-Cartan $Y$, onde:

$$
\mathcal{G}(u, v, \varepsilon ; q)=L(u, v, \varepsilon)+M(u, v, \varepsilon) q+N(u, v, \varepsilon) q^{2}=0
$$

$L, M, N$ dadas pelas expressões da equação 2.39 e

$$
Y=q \mathcal{G}_{q} \frac{\partial}{\partial u}+\mathcal{G}_{q} \frac{\partial}{\partial v}-\left(\mathcal{G}_{v}+q \mathcal{G}_{u}\right) \frac{\partial}{\partial q} .
$$

Lembremos que as singularidades do campo $Y$ são $(0,0 ; q)$, sendo $q$ as raízes do polinômio cúbico $\Psi(q)=-\left(\mathcal{G}_{v}+q \mathcal{G}_{u}\right)$.

Logo,

$$
\Psi(q)=-\left(\mathcal{G}_{v}+q \mathcal{G}_{u}\right)=0 \Longleftrightarrow q^{3}+A_{2} q^{2}+A_{1} q+A_{0}=0,
$$

onde

$$
\begin{aligned}
& A_{2}=\frac{2\left[a\left(k_{2}^{+}-k_{2}^{-}\right)+b\left(k_{2}^{-}-k_{1}\right)-\beta\left(k_{2}^{+}-k_{1}\right)\right]}{\gamma\left(k_{1}-k_{2}^{+}\right)+c\left(k_{2}^{-}-k_{1}\right)}, \\
& A_{1}=\frac{(d-2 c)\left(k_{2}^{-}-k_{1}\right)+(\rho-2 \gamma)\left(k_{1}-k_{2}^{+}\right)}{\gamma\left(k_{1}-k_{2}^{+}\right)+c\left(k_{2}^{-}-k_{1}\right)}-\frac{3 \phi^{\prime}(0)\left(k_{2}^{+}-k_{2}^{-}\right)^{2}}{\varepsilon\left[\gamma\left(k_{1}-k_{2}^{+}\right)+c\left(k_{2}^{-}-k_{1}\right)\right]}, \\
& A_{0}=\frac{\beta\left(k_{2}^{+}-k_{1}\right)+b\left(k_{1}-k_{2}^{-}\right)}{\gamma\left(k_{1}-k_{2}^{+}\right)+c\left(k_{2}^{-}-k_{1}\right)} .
\end{aligned}
$$


Observe que os denominadores dos coeficientes $A_{2}, A_{1}$ e $A_{0}$ dados em 2.40 são não nulos, pela condição de transversalidade em 2.36.

O discriminante $\Delta$ da equação cúbica $q^{3}+A_{2} q^{2}+A_{1} q+A_{0}=0$ é dado por:

$$
\Delta=\frac{1}{4}\left(A_{0}+\frac{2 A_{2}^{3}}{27}-\frac{A_{2} A_{1}}{3}\right)^{2}+\frac{1}{27}\left(A_{1}-\frac{A_{2}^{2}}{3}\right)^{3} \text {. }
$$

Portanto, para $\varepsilon>0$ suficientemente pequeno, o sinal de $\Delta$ é determinado pelo sinal de $\gamma\left(k_{1}-k_{2}^{+}\right)+c\left(k_{2}^{-}-k_{1}\right)$, pois a segunda parcela do coeficiente $A_{1}$ na equação 2.40 é a única que contém $\varepsilon$ no denominador e, como já observamos, $\phi^{\prime}(0)>0$. Assim,

$$
\begin{aligned}
& \Delta>0 \Longleftrightarrow \gamma\left(k_{1}-k_{2}^{+}\right)+c\left(k_{2}^{-}-k_{1}\right)<0 \Longleftrightarrow \gamma<c\left(\frac{k_{1}-k_{2}^{-}}{k_{1}-k_{2}^{+}}\right), \\
& \Delta<0 \Longleftrightarrow \gamma\left(k_{1}-k_{2}^{+}\right)+c\left(k_{2}^{-}-k_{1}\right)>0 \Longleftrightarrow \gamma>c\left(\frac{k_{1}-k_{2}^{-}}{k_{1}-k_{2}^{+}}\right) .
\end{aligned}
$$

Logo, se $\Delta>0, \Psi(q)$ possui uma única raiz real denotada por $q=q_{1}$ e se $\Delta<0$, $\Psi(q)$ possui três raízes reais distintas $q$, sendo $q \in\left\{q_{1}, q_{2}, q_{3}\right\}$. Veja figura 2.16, na qual podemos observar que a reta pontilhada de equação $\gamma=c\left(\frac{k_{1}-k_{2}^{-}}{k_{1}-k_{2}^{+}}\right)$tem coeficiente angular negativo pois, por hipótese, $k_{2}^{+}<k_{1}<k_{2}^{-}$.

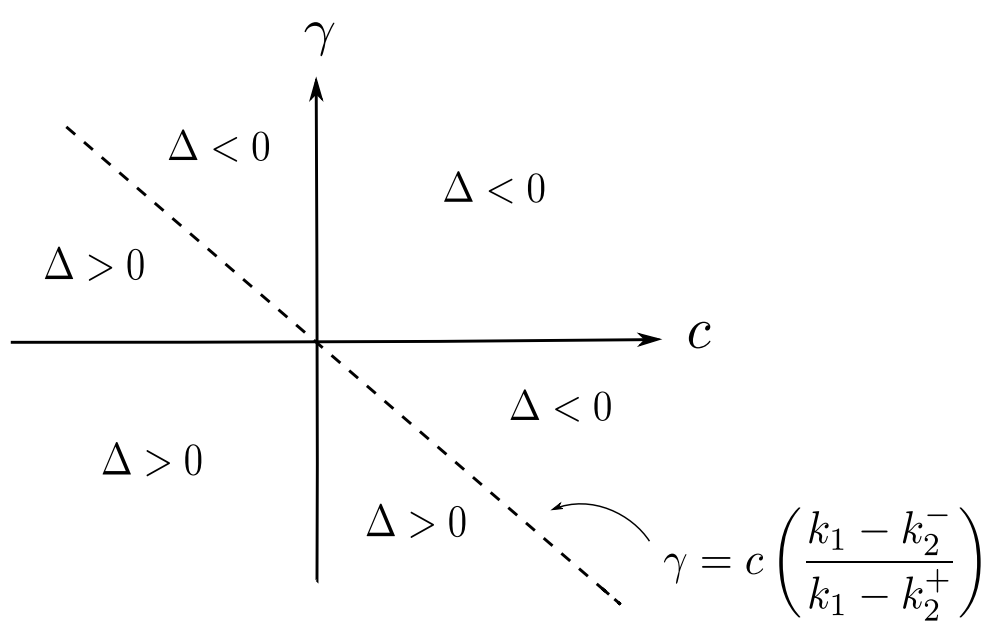

Figura 2.16: Regiões do plano c $\gamma$ onde temos uma única raiz real $(\Delta>0)$ ou três raízes reais distintas $(\Delta<0)$.

Passemos agora a estudar a natureza dos equilíbrios $(0,0 ; q), q \in\left\{q_{1}, q_{2}, q_{3}\right\}$ nos casos em que $\Delta>0$ e $\Delta<0$.

A linearização do campo $Y=\left(Y_{1}, Y_{2}, Y_{3}\right)=\left(q \mathcal{G}_{q}, \mathcal{G}_{q},-\left(\mathcal{G}_{v}+q \mathcal{G}_{u}\right)\right)$ em $(0,0 ; q)$, 
$q \in\left\{q_{1}, q_{2}, q_{3}\right\}$ é:

$$
D Y(0,0 ; q)=\left[\begin{array}{ccc}
\frac{\partial Y_{1}}{\partial u}(0,0 ; q) & \frac{\partial Y_{1}}{\partial v}(0,0 ; q) & 0 \\
\frac{\partial Y_{2}}{\partial u}(0,0 ; q) & \frac{\partial Y_{2}}{\partial v}(0,0 ; q) & 0 \\
0 & 0 & \Psi^{\prime}(q)
\end{array}\right]
$$

sendo

$$
\begin{aligned}
\frac{\partial Y_{1}}{\partial u}(0,0 ; q) & =2 \varepsilon\left[c\left(k_{1}-k_{2}^{-}\right)-\gamma\left(k_{1}-k_{2}^{+}\right)\right] q^{2}+ \\
& +\varepsilon\left[a\left(k_{2}^{-}-k_{2}^{+}\right)+\beta\left(k_{2}^{+}-k_{1}\right)+b\left(k_{1}-k_{2}^{-}\right)\right] q \\
\frac{\partial Y_{1}}{\partial v}(0,0 ; q) & =2 \varepsilon\left[b\left(k_{1}-k_{2}^{-}\right)-\beta\left(k_{1}-k_{2}^{+}\right)\right] q^{2}+ \\
& +\left\{\varepsilon\left[(d-c)\left(k_{1}-k_{2}^{-}\right)+(\gamma-\rho)\left(k_{1}-k_{2}^{+}\right)\right]+3 \phi^{\prime}(0)\left(k_{2}^{+}-k_{2}^{-}\right)^{2}\right\} q \\
\frac{\partial Y_{2}}{\partial u}(0,0 ; q) & =2 \varepsilon\left[c\left(k_{1}-k_{2}^{-}\right)-\gamma\left(k_{1}-k_{2}^{+}\right)\right] q+ \\
& +\varepsilon\left[a\left(k_{2}^{-}-k_{2}^{+}\right)+\beta\left(k_{2}^{+}-k_{1}\right)+b\left(k_{1}-k_{2}^{-}\right)\right] \\
\frac{\partial Y_{2}}{\partial v}(0,0 ; q) & =2 \varepsilon\left[b\left(k_{1}-k_{2}^{-}\right)-\beta\left(k_{1}-k_{2}^{+}\right)\right] q+ \\
& +\varepsilon\left[(d-c)\left(k_{1}-k_{2}^{-}\right)+(\gamma-\rho)\left(k_{1}-k_{2}^{+}\right)\right]+3 \phi^{\prime}(0)\left(k_{2}^{+}-k_{2}^{-}\right)^{2} \\
& +\varepsilon\left[(d-2 c)\left(k_{2}^{-}-k_{1}\right)+(\rho-2 \gamma)\left(k_{1}-k_{2}^{+}\right)\right]-3 \phi^{\prime}(0)\left(k_{2}^{+}-k_{2}^{-}\right)^{2} \\
\Psi^{\prime}(q) & =3 \varepsilon\left[\gamma\left(k_{1}-k_{2}^{+}\right)-c\left(k_{1}-k_{2}^{-}\right)\right] q^{2}+ \\
& +2 \varepsilon\left[a\left(k_{2}^{+}-k_{2}^{-}\right)+2 \beta\left(k_{1}-k_{2}^{+}\right)-2 b\left(k_{1}-k_{2}^{-}\right)\right] q+ \\
& \\
& \\
& \\
&
\end{aligned}
$$

Os autovalores de $D Y(0,0 ; q)$ são $\lambda_{1}=0, \lambda_{2}:=\Psi^{\prime}(q)$ e $\lambda_{3}:=\lambda_{3}(q), q \in\left\{q_{1}, q_{2}, q_{3}\right\}$, 
onde

$$
\begin{aligned}
\lambda_{3} & =2 \varepsilon\left[c\left(k_{1}-k_{2}^{-}\right)-\gamma\left(k_{1}-k_{2}^{+}\right)\right] q^{2}+ \\
& +\varepsilon\left[a\left(k_{2}^{-}-k_{2}^{+}\right)+3 \beta\left(k_{2}^{+}-k_{1}\right)+3 b\left(k_{1}-k_{2}^{-}\right)\right] q+ \\
& +\varepsilon\left[(d-c)\left(k_{1}-k_{2}^{-}\right)+(\gamma-\rho)\left(k_{1}-k_{2}^{+}\right)\right]+3 \phi^{\prime}(0)\left(k_{2}^{+}-k_{2}^{-}\right)^{2}, q \in\left\{q_{1}, q_{2}, q_{3}\right\} .
\end{aligned}
$$

Como $q \in\left\{q_{1}, q_{2}, q_{3}\right\}$ não é raiz repetida do polinômio $\Psi(q)$, segue que $\Psi^{\prime}(q) \neq 0$, para todo $q \in\left\{q_{1}, q_{2}, q_{3}\right\}$ e, portanto, $\lambda_{2} \neq 0$.

Para o caso em que $\Delta>0$, o equilíbrio $\left(0,0 ; q_{1}\right)$ do campo de Lie-Cartan $Y$ é uma sela. De fato, pelas expressões de $\lambda_{2}=\lambda_{2}\left(q_{1}\right)$ em 2.41 e $\lambda_{3}=\lambda_{3}\left(q_{1}\right)$ em 2.42 temos, para $\varepsilon>0$ suficientemente pequeno, que $\frac{\lambda_{2}}{\lambda_{3}}<0$, pois

$$
\lim _{\varepsilon \rightarrow 0} \frac{\lambda_{2}\left(q_{1}\right)}{\lambda_{3}\left(q_{1}\right)}=-1<0
$$

Portanto, para $\Delta>0$, que corresponde ao caso em que $\gamma<c\left(\frac{k_{1}-k_{2}^{-}}{k_{1}-k_{2}^{+}}\right)$(veja figura 2.16), o ponto umbílico $(0,0)$ é Darbouxiano do tipo $D_{1}$.

Analogamente, para o caso em que $\Delta<0$, os equilíbrios $\left(0,0 ; q_{i}\right), i \in\{1,2,3\}$, do campo de Lie-Cartan $Y$ são selas. De fato, pelas expressões de $\lambda_{2}=\lambda_{2}\left(q_{i}\right)$ em 2.41) e $\lambda_{3}=\lambda_{3}\left(q_{i}\right)$ em 2.42 temos, para $\varepsilon>0$ suficientemente pequeno, que $\frac{\lambda_{2}}{\lambda_{3}}<0$, pois

$$
\lim _{\varepsilon \rightarrow 0} \frac{\lambda_{2}\left(q_{i}\right)}{\lambda_{3}\left(q_{i}\right)}=-1<0, i \in\{1,2,3\}
$$

Assim, para $\Delta<0$, que corresponde ao caso em que $\gamma>c\left(\frac{k_{1}-k_{2}^{-}}{k_{1}-k_{2}^{+}}\right)$(veja figura 2.16, o ponto umbílico $(0,0)$ é Darbouxiano do tipo $D_{3}$.

Isto completa a demonstração do teorema 2.2.6.

As figuras 2.17 e 2.18, abaixo, representam os pontos de tangências quadráticas da figura 2.3 (à esquerda), e suas $\varepsilon$-regularizações (à direita). A região de regularização foi feita em grande escala para facilitar a visualização das linhas de curvatura principal da superfície regularizada, $\mathbf{S}_{\varepsilon}$, na vizinhança da origem. 


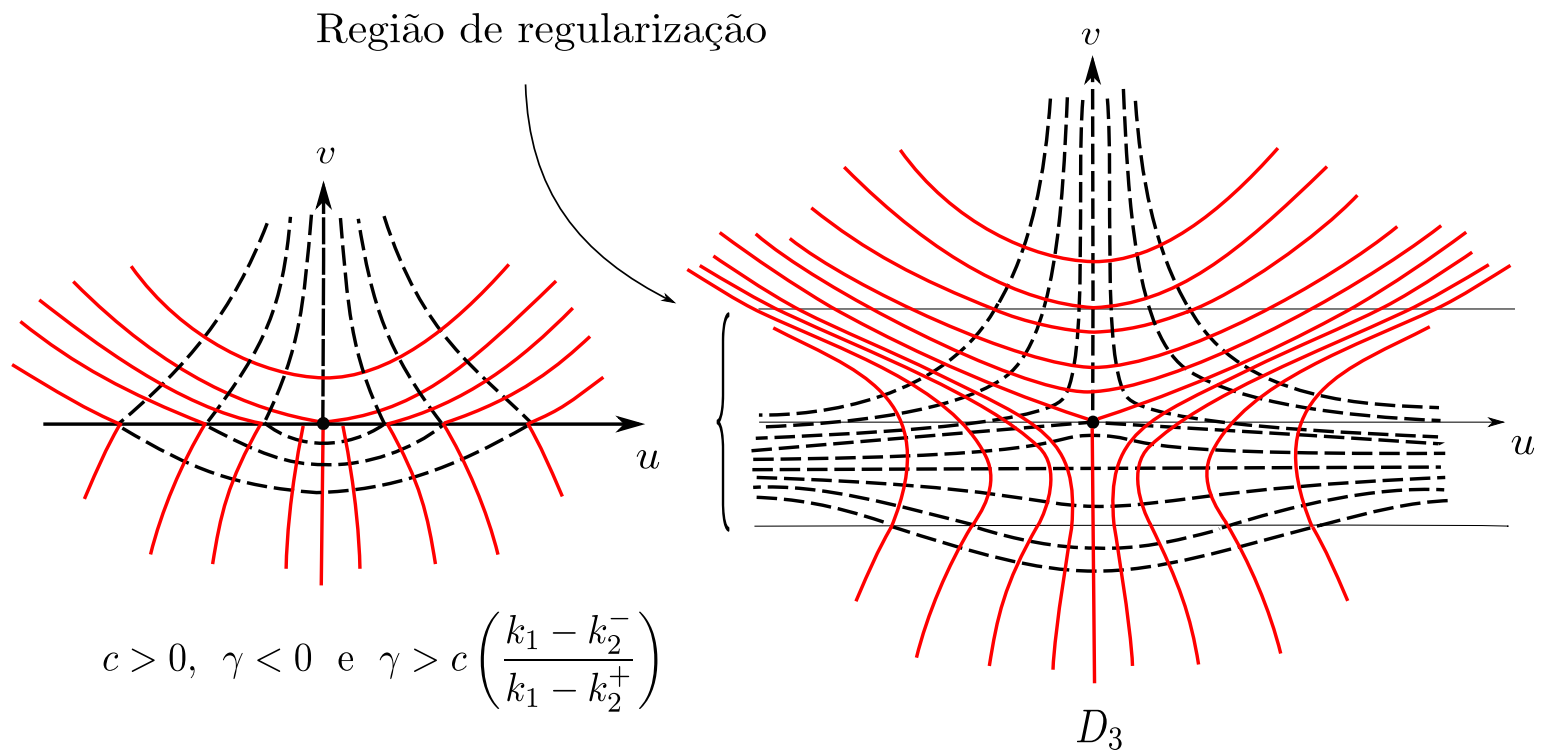

Figura 2.17: À esquerda, temos que a origem é um ponto de tangência tal que $k_{2}^{+}<k_{1}<k_{2}^{-}$, $c>0, \quad \gamma>0$ e $\gamma>c\left(\frac{k_{1}-k_{2}^{-}}{k_{1}-k_{2}^{+}}\right)$e, à direita, sua $\varepsilon$-regularização. Linhas principais mínimas (linhas tracejadas) são deslizantes e as linhas principais máximas (linhas contínuas) costuram.

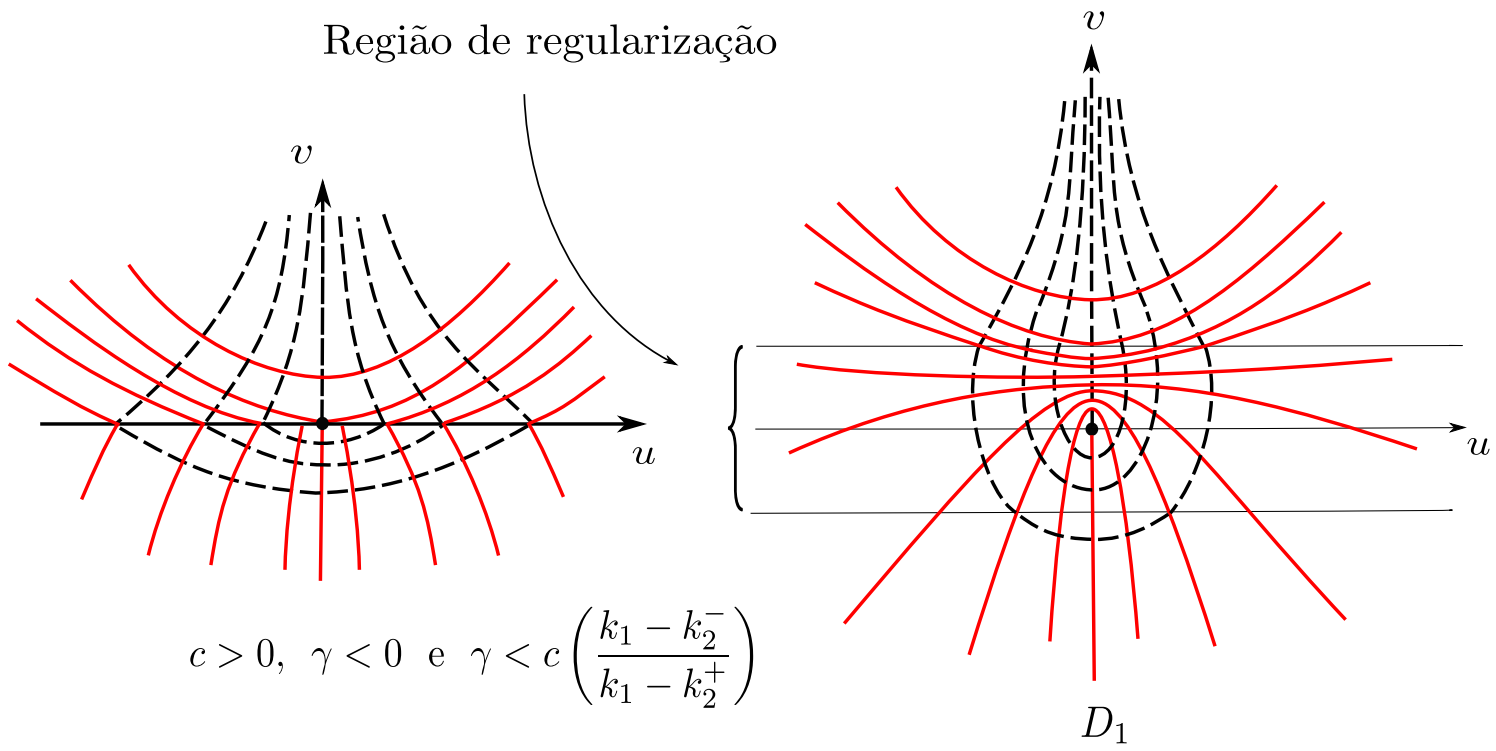

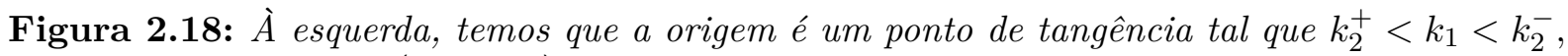
$c>0, \quad \gamma>0$ e $\gamma<c\left(\frac{k_{1}-k_{2}^{-}}{k_{1}-k_{2}^{+}}\right)$e, à direita, sua $\varepsilon$-regularização. Linhas principais máximas (linhas contínuas) são deslizantes e as linhas principais minimas (linhas tracejadas) costuram.

Observação 2.2.7. Vimos na demonstração do teorema 2.2.6 que na carta $q=d v / d u$, se $\Delta<0$ então $q_{1}, q_{2}$ e $q_{3}$ denotam as três raízes distintas do polinômio

$$
q^{3}+A_{2} q^{2}+A_{1} q+A_{0}=0
$$

$\left(A_{i}\right.$ definidos em (2.40)). Observemos que, para $\varepsilon>0$ pequeno o suficiente, o coeficiente 
$A_{1}$ torna-se cada vez maior. Portanto, podemos dizer que uma das raizes, digamos $q_{1}$, pode ser considerada nula, $q_{1}=0$, e as outras duas raízes $q_{2}$ e $q_{3}$ dadas por $q_{2}=\infty$ e $q_{3}=-\infty$. Na figura 2.17 (à direita), fizemos uma das separatrizes do ponto umbílicos $D_{3}$ sobre o eixo-v (que corresponde a $q_{1}=0$ ) e as outras duas "quase" paralelas ao eixo$u$ (correspondentes a $q_{2}=\infty$ e $\left.q_{3}=-\infty\right)$. Uma observação análoga vale para a única separatriz $(q=0)$ do ponto umbílico Darbouxiano $D_{1}$ esboçada na figura 2.18 (à direita) sobre o eixo-v.

\subsection{2 $2^{\circ}$ Caso: Regularização na Vizinhança de um Ponto de Cruzamento Transversal $\left(k_{3}^{+} \cdot k_{3}^{-} \neq 0\right)$}

Nesta seção estabeleceremos as hipóteses para que do ponto $\delta(0)$, de cruzamento transversal das linhas de curvatura das superfícies $\mathbf{S}^{+}$e $\mathbf{S}^{-}$com o bordo $\boldsymbol{\delta}$, emerjam pontos umbílicos sobre a superfície regularizada $\mathbf{S}_{\varepsilon}, \varepsilon>0$ suficientemente pequeno. $\mathrm{O}$ principal resultado obtido foi o teorema 2.2 .10 .

Das proposições 2.1.1 e 2.1.3 da seção 2.1.1 sabemos que as linhas de curvatura principal das superfícies $\mathbf{S}^{+}$e $\mathbf{S}^{-}$cruzam transversalmente o bordo $\boldsymbol{\delta}$ em uma vizinhança da origem $\delta(0)$ se, e somente se, $k_{3}^{+} \cdot k_{3}^{-} \neq 0$.

Duas condições foram estabelecidas na seção 2.1 .2 pela proposições 2.1 .8 e 2.1 .9 para que $\delta(0)$ seja um ponto de transição e, para as linhas de curvatura principal mínima, $\delta(u)$ seja um ponto de costura se $u>0$ e de deslizamento se $u<0$.

O próximo lema 2.2.8 apresenta condições relativas à função de transição $\phi$ para que a origem seja um ponto umbílico da superfície regularizada $\mathbf{S}_{\varepsilon}$, para $\varepsilon>0$ suficientemente pequeno.

Lema 2.2.8. Suponha que $\frac{k_{2}^{+}-k_{1}}{k_{3}^{+}}=\frac{k_{2}^{-}-k_{1}}{k_{3}^{-}}$e $k_{3}^{+}<0<k_{3}^{-}$, ou seja, as linhas de curvatura principal das superfícies $\mathbf{S}^{+}$e $\mathbf{S}^{-}$cruzam o bordo $\boldsymbol{\delta}$ transversalmente em uma vizinhança da origem $\delta(0)$. Sejam $\phi$ uma função de transição e $\mathbf{S}_{\varepsilon}$ a superfície regularizada, $\varepsilon>0$ pequeno o suficiente.

$$
\begin{aligned}
& \text { Se } k_{2}^{+}<k_{1}<k_{2}^{-} \quad \text { e } \phi(0)=\frac{k_{2}^{-}-k_{1}}{k_{2}^{-}-k_{2}^{+}} \text {então o conjunto } \\
& \quad U_{\varepsilon}=\{(u, v, \varepsilon): L(u, v, \varepsilon)=M(u, v, \varepsilon)=0\}
\end{aligned}
$$

é uma curva regular tal que $X\left(U_{\varepsilon}\right)=\{(0,0,0)\}$ é ponto umbilico de $\mathbf{S}_{\varepsilon}$.

Demonstração. Da hipótese $\frac{k_{2}^{+}-k_{1}}{k_{3}^{+}}=\frac{k_{2}^{-}-k_{1}}{k_{3}^{-}}$e $k_{3}^{+}<0<k_{3}^{-}$podemos concluir que $k_{1}=\frac{k_{3}^{+} k_{2}^{-}-k_{2}^{+} k_{3}^{-}}{k_{3}^{+}-k_{3}^{-}}$. Agora, substituindo em $\phi(0)=\frac{k_{2}^{-}-k_{1}}{k_{2}^{-}-k_{2}^{+}}$essa expressão obtida para 
$k_{1}$, obtemos que $\phi(0)=\frac{k_{3}^{-}}{k_{3}^{-}-k_{3}^{+}}$.

As expressões para $L$ e $M$ foram obtidas em (2.34) e são dadas por:

$$
\begin{aligned}
L(u, w, \varepsilon) & =-\varepsilon^{4}\left\{k_{3}^{-}+\phi(w)\left(k_{3}^{+}-k_{3}^{-}\right)+[\gamma+\phi(w)(c-\gamma)] u+\right. \\
& \left.+\left[\varepsilon(\beta+\phi(w)(b-\beta))+\phi^{\prime}(w)\left(k_{3}^{+}-k_{3}^{-}\right)\right] w\right\}+O(2), \\
M(u, w, \varepsilon) & =\varepsilon^{2}\left\{\varepsilon\left[k_{2}^{-}-k_{1}+\phi(w)\left(k_{2}^{+}-k_{2}^{-}\right)\right]+[\varepsilon(\beta-a+\phi(w)(b-\beta))+\right. \\
& \left.+2 \phi^{\prime}(w)\left(k_{3}^{+}-k_{3}^{-}\right)\right] u+\varepsilon[\varepsilon \rho-\varepsilon \gamma+\varepsilon \phi(w)(\gamma-c+d-\rho)+ \\
& \left.\left.+2 \phi^{\prime}(w)\left(k_{2}^{+}-k_{2}^{-}\right)\right] w\right\}+O(2) .
\end{aligned}
$$

Portanto, considerando $(u, w)=(0,0)$ e $\phi(0)=\frac{k_{3}^{-}}{k_{3}^{-}-k_{3}^{+}}$em $L$ obtemos $L(0,0, \varepsilon)=0$, $\varepsilon>0$ pequeno. E, analogamente, se $(u, w)=(0,0)$ e $\phi(0)=\frac{k_{2}^{-}-k_{1}}{k_{2}^{-}-k_{2}^{+}}$em $M$, então $M(0,0, \varepsilon)=0, \quad \varepsilon>0$. Donde concluímos que em $(u, w)=(0,0)$ temos um ponto umbílico.

Retornando às coordenadas originais $(u, v, \varepsilon)$, lembrando que $v=\varepsilon w, \varepsilon>0$ suficientemente pequeno, segue que $w=\varepsilon^{-1} v$ e a curva de umbílicos é dada por

$$
U(\varepsilon)=\{(0,0, \varepsilon), \varepsilon>0\}
$$

Isto completa a demonstração.

Observação 2.2.9. Vimos na observação 2.2.4 que:

$$
k_{2}^{+}<k_{1}<k_{2}^{-} \Longrightarrow \phi(0)=\frac{k_{2}^{-}-k_{1}}{k_{2}^{-}-k_{2}^{+}} \in(0,1) .
$$

Além disso,

$$
k_{3}^{+}<0<k_{3}^{-} \Longrightarrow \phi(0)=\frac{k_{3}^{-}}{k_{3}^{-}-k_{3}^{+}} \in(0,1) \text {. }
$$

O principal resultado obtido nessa seção é o próximo teorema que classifica o ponto umbílico do lema 2.2.8.

Teorema 2.2.10. Considere a superfície $\mathbf{S}=\mathbf{S}^{+} \cup \boldsymbol{\delta} \cup \mathbf{S}^{-}$e sua $\varepsilon$-regularização $\mathbf{S}_{\varepsilon}$. Suponha que a origem $\delta(0)$ seja um ponto de cruzamento transversal tal que $k_{3}^{+}<0<k_{3}^{-}$, 


$$
k_{2}^{+}<k_{1}<k_{2}^{-}, \quad \frac{k_{2}^{+}-k_{1}}{k_{3}^{+}}=\frac{k_{2}^{-}-k_{1}}{k_{3}^{-}} \quad \text { e } k_{3}^{-}>\frac{\left(k_{1}-k_{2}^{-}\right)\left[\gamma\left(k_{1}-k_{2}^{-}\right)+c\left(k_{1}-k_{2}^{-}\right)\right]}{(a-b)\left(k_{1}-k_{2}^{-}\right)+(a-\beta)\left(k_{1}-k_{2}^{+}\right)} \text {. }
$$

Se $\phi(0)=\frac{k_{2}^{-}-k_{1}}{k_{2}^{-}-k_{2}^{+}} \quad$ então sobre $\mathbf{S}_{\varepsilon}$ a origem será um ponto umbílico Darbouxiano do tipo $D_{3}$, para $\varepsilon>0$ suficientemente pequeno.

Demonstração. Os pontos umbílicos da superfície regularizada $\mathbf{S}_{\varepsilon}$ são dados pelas equações $L(u, w, \varepsilon)=M(u, w, \varepsilon)=N(u, w, \varepsilon)=0$.

Pelo lema 2.2.8, considerando $\phi(0)=\frac{k_{2}^{-}-k_{1}}{k_{2}^{-}-k_{2}^{+}}=\frac{k_{3}^{-}}{k_{3}^{-}-k_{3}^{+}}$, na vizinhança do ponto umbílico $(0,0)$, as expressões 2.34 de $L, M$ e $N$ tornam-se:

$$
\begin{aligned}
L(u, w, \varepsilon) & =\frac{\varepsilon^{4}\left(c k_{3}^{-}-\gamma k_{3}^{+}\right)}{k_{3}^{+}-k_{3}^{-}} u+\frac{\varepsilon^{4}}{k_{3}^{+}-k_{3}^{-}}\left[\varepsilon\left(b k_{3}^{-}-\beta k_{3}^{+}\right)-\right. \\
& \left.-2 \phi^{\prime}(0)\left(k_{3}^{+}-k_{3}^{-}\right)^{2}\right] w+O(2) \\
M(u, w, \varepsilon)= & \left\{\frac{\varepsilon^{3}\left[k_{3}^{+}(\beta-a)+k_{3}^{-}(a-b)\right]}{k_{3}^{+}-k_{3}^{-}}+2 \varepsilon^{2} \phi^{\prime}(0)\left(k_{3}^{+}-k_{3}^{-}\right)^{2}\right\} u+ \\
& +\left\{\frac{\varepsilon^{4}\left[k_{3}^{+}(\rho-\gamma)+k_{3}^{-}(c-d)\right]}{k_{3}^{+}-k_{3}^{-}}+3 \varepsilon^{3} \phi^{\prime}(0)\left(k_{2}^{+}-k_{2}^{-}\right)\left(k_{3}^{+}-k_{3}^{-}\right)\right\} w+O(2), \\
N(u, w, \varepsilon)= & \frac{\varepsilon^{2}\left(\gamma k_{3}^{+}-c k_{3}^{-}\right)}{k_{3}^{+}-k_{3}^{-}} u+\frac{\varepsilon^{2}}{k_{3}^{+}-k_{3}^{-}}\left[\varepsilon\left(\beta k_{3}^{+}-b k_{3}^{-}\right)+\right. \\
& \left.+2 \phi^{\prime}(0)\left(k_{3}^{+}-k_{3}^{-}\right)^{2}\right] w+O(2) .
\end{aligned}
$$

A Condição de Transversalidade T: Vamos calcular o determinante Jacobiano das funções $L(u, w, \varepsilon)$ e $M(u, w, \varepsilon)$ em $(u, w)=(0,0)$, usando as expressões de $L$ e $M$ obtidas em 2.43).

$$
\left.\operatorname{det}\left(\frac{\partial(L, M)}{\partial(u, w)}\right)\right|_{u=w=0}=\varepsilon^{6}\left[4 \phi^{\prime}(0)^{2}\left(k_{3}^{+}-k_{3}^{-}\right)^{2}\right]+O(\varepsilon, 7)
$$

Portanto, para $\varepsilon>0$ suficientemente pequeno, $\left.\operatorname{det}\left(\frac{\partial(L, M)}{\partial(u, w)}\right)\right|_{u=w=0} \neq 0$, pois $\phi^{\prime}(0)>0$ e, por hipótese, $k_{3}^{+} \neq k_{3}^{-}$.

A Condição do Discriminante D: Para $(u, w)$ próximo ao ponto umbílico $(0,0)$, a 
equação diferencial das linhas de curvatura é dada por:

$$
L(u, w, \varepsilon) d w^{2}+M(u, w, \varepsilon) d u d w+N(u, w, \varepsilon) d u^{2}=0,
$$

sendo as expressões de $L, M$ e $N$ obtidas em 2.43.

Vamos escrever a equação 2.44 nas coordenadas originais $(u, v, \varepsilon)$, lembrando que $v=\varepsilon w, \varepsilon>0$ suficientemente pequeno. Logo, ao substituirmos as expressões $w=v / \varepsilon$ e $d w=d v / \varepsilon$ na equação (2.44) obtemos, na carta $(u, v)$ a seguinte equação diferencial para as linhas de curvatura:

$$
L(u, v, \varepsilon) d v^{2}+M(u, v, \varepsilon) d u d v+N(u, v, \varepsilon) d u^{2}=0
$$

onde

$$
\begin{aligned}
L(u, v, \varepsilon) & =\varepsilon\left(c k_{3}^{-}-\gamma k_{3}^{+}\right) u+\left[\varepsilon\left(b k_{3}^{-}-\beta k_{3}^{+}\right)-2 \phi^{\prime}(0)\left(k_{3}^{+}-k_{3}^{-}\right)^{2}\right] v+O(2), \\
M(u, v, \varepsilon) & =\left\{\varepsilon\left[k_{3}^{+}(\beta-a)+k_{3}^{-}(a-b)\right]+2 \phi^{\prime}(0)\left(k_{3}^{+}-k_{3}^{-}\right)^{2}\right\} u+ \\
& +\left\{\varepsilon\left[k_{3}^{-}(c-d)+k_{3}^{+}(\rho-\gamma)\right]+3 \phi^{\prime}(0)\left(k_{3}^{+}-k_{3}^{-}\right)\left(k_{2}^{+}-k_{2}^{-}\right)\right\} v+O(2), \\
N(u, v, \varepsilon) & =\varepsilon\left(\gamma k_{3}^{+}-c k_{3}^{-}\right) u+\left[\varepsilon\left(\beta k_{3}^{+}-b k_{3}^{-}\right)+2 \phi^{\prime}(0)\left(k_{3}^{+}-k_{3}^{-}\right)^{2}\right] v+O(2) .
\end{aligned}
$$

Na carta $(u, v ; p)$, sendo $p=d v / d u$, vamos considerar a superfície de Lie-Cartan $\mathcal{L}^{-1}\{0\}$ sobre a qual temos definido o campo de Lie-Cartan $X$, sendo:

$$
\mathcal{L}(u, v, \varepsilon ; p)=L(u, v, \varepsilon) p^{2}+M(u, v, \varepsilon) p+N(u, v, \varepsilon)=0
$$

$L, M, N$ dadas pelas expressões da equação 2.46 e

$$
X=\mathcal{L}_{p} \frac{\partial}{\partial u}+p \mathcal{L}_{p} \frac{\partial}{\partial v}-\left(\mathcal{L}_{u}+p \mathcal{L}_{v}\right) \frac{\partial}{\partial p}
$$

Lembremos que as singularidades de $X$ são $(0,0 ; p)$, sendo $p$ as raízes do polinômio cúbico $\Psi(p)=-\left(\mathcal{L}_{u}+p \mathcal{L}_{v}\right)$.

Logo,

$$
\Psi(p)=-\left(\mathcal{L}_{u}+p \mathcal{L}_{v}\right)=0 \Longleftrightarrow p^{3}+A_{2} p^{2}+A_{1} p+A_{0}=0 .
$$

Os coeficientes $A_{2}, A_{1}$ e $A_{0}$ do polinômio $\Psi(p)$ são dados por:

$$
A_{2}=\frac{\varepsilon\left[k_{3}^{-}(d-2 c)+k_{3}^{+}(2 \gamma-\rho)\right]-3 \phi^{\prime}(0)\left(k_{2}^{+}-k_{2}^{-}\right)\left(k_{3}^{+}-k_{3}^{-}\right)}{\varepsilon\left(\beta k_{3}^{+}-b k_{3}^{-}\right)+2 \phi^{\prime}(0)\left(k_{3}^{+}-k_{3}^{-}\right)^{2}},
$$




$$
\begin{aligned}
& A_{1}=\frac{\varepsilon\left[k_{3}^{+}(a-2 \beta)+k_{3}^{-}(2 b-a)\right]-4 \phi^{\prime}(0)\left(k_{3}^{+}-k_{3}^{-}\right)^{2}}{\varepsilon\left(\beta k_{3}^{+}-b k_{3}^{-}\right)+2 \phi^{\prime}(0)\left(k_{3}^{+}-k_{3}^{-}\right)^{2}}, \\
& A_{0}=\frac{\varepsilon\left(c k_{3}^{-}-\gamma k_{3}^{+}\right)}{\varepsilon\left(\beta k_{3}^{+}-b k_{3}^{-}\right)+2 \phi^{\prime}(0)\left(k_{3}^{+}-k_{3}^{-}\right)^{2}} .
\end{aligned}
$$

Para $\varepsilon>0$ suficientemente pequeno, observamos das expressões 2.47) que o polinômio $p^{3}+A_{2} p^{2}+A_{1} p+A_{0}=0$ possui três raízes reais distintas que serão representadas por $p_{0}$, $p_{1}$ e $p_{2}$. Além disso, $p_{0}$ pode ser considerada tão próxima de zero e $p_{1} \cdot p_{2}$ tão próximo de $\frac{-4 \phi^{\prime}(0)\left(k_{3}^{+}-k_{3}^{-}\right)^{2}}{2 \phi^{\prime}(0)\left(k_{3}^{+}-k_{3}^{-}\right)^{2}}=-2$ quanto quisermos, bastando escolher $\varepsilon>0$ pequeno o suficiente. Portanto, $p_{1} \cdot p_{2}<-1$ e o ponto umbílico $(0,0)$ é Darbouxiano do tipo $D_{3}$.

Isto completa a demonstração.

A figura 2.19 (à esquerda) representa um ponto de cruzamento transversal e sua $\varepsilon$-regularização (à direita), na qual a origem é um ponto umbílico Darbouxiano do tipo $D_{3}$. A região de regularização foi feita em grande escala para facilitar a visualização das linhas de curvatura principal da superfície regularizada, $\mathbf{S}_{\varepsilon}$, na vizinhança da origem.

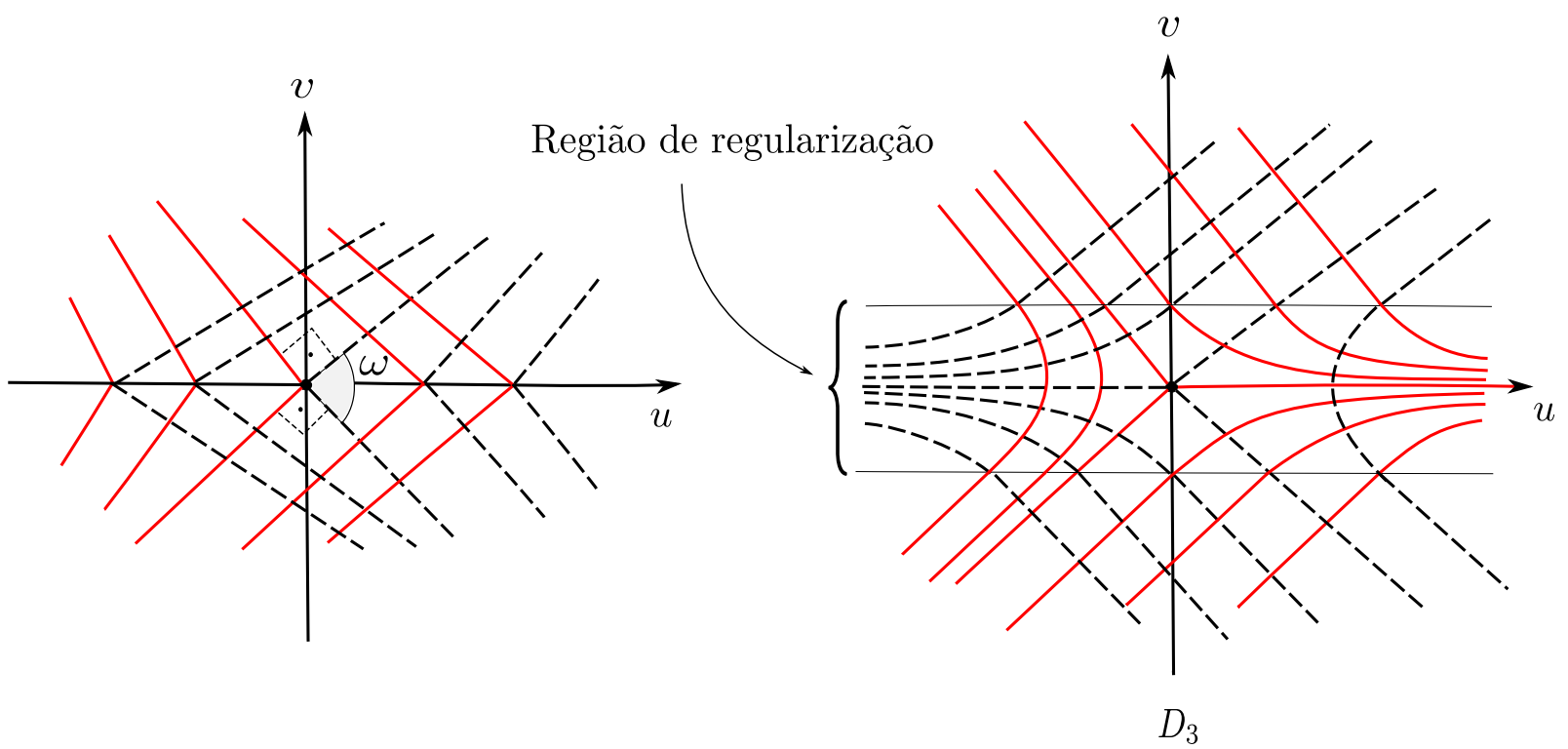

Figura 2.19: $\grave{A}$ esquerda, temos que a origem é um ponto de cruzamento transversal (observe que devemos ter $\omega=90^{\circ}$ ) e, à direita, sua $\varepsilon$-regularização. Linhas principais mínimas (linhas tracejadas) são deslizantes se $u<0$ e costuram se $u>0$. As linhas principais máximas (linhas contínuas) costuram se $u<0$ e deslizam se $u>0$. 


\subsection{3 $3^{\circ}$ Caso: Regularização na Vizinhança de um Ponto de Dobra}$$
\left(k_{3}^{+}=0 \text { e } k_{3}^{-} \neq 0\right)
$$

O último caso a ser considerado é a regularização de um ponto de $\boldsymbol{\delta}$-dobra (definição 2.1.10. Nesta seção veremos que sendo $\delta(0)$ um ponto de $\boldsymbol{\delta}$-dobra, sobre a superfície regularizada $\mathbf{S}_{\varepsilon}$ não teremos pontos umbílicos em uma vizinhança da origem, para todo $\varepsilon$ positivo. O principal resultado desta seção é o teorema 2.2.11 seguinte.

Das proposições 2.1.3 e 2.1.1 da seção 2.1.1 sabemos que $\delta(0)$ é um ponto de cruzamento transversal para $\mathbf{S}^{-}$se, e somente se, $k_{3}^{-} \neq 0$ e um ponto de tangência quadrática para $\mathbf{S}^{+}$se, e somente se, $k_{3}^{+}=0$.

Teorema 2.2.11. Considere a superfície $\mathbf{S}=\mathbf{S}^{+} \cup \boldsymbol{\delta} \cup \mathbf{S}^{-}$e sua $\varepsilon$-regularização $\mathbf{S}_{\varepsilon}$. Suponha que a origem $\delta(0)$ seja um ponto de $\boldsymbol{\delta}$-dobra tal que $k_{3}^{+}=0, k_{3}^{-} \neq 0$ e seja $\phi$ uma função de transição. Então sobre $\mathbf{S}_{\varepsilon}$ não teremos pontos umbílicos em uma vizinhança da origem, para todo $\varepsilon>0$.

Demonstração. Considerando $k_{3}^{+}=0$ e $k_{3}^{-} \neq 0$ na parametrização para $\mathbf{S}_{\varepsilon}$ na carta $(u, v)$ dada em (2.28), obtemos as seguintes expressões para as funções $L, M$ e $N$ :

$$
\begin{aligned}
L(u, v, \varepsilon) & =k_{3}^{-}\left(\phi_{\varepsilon}(v)-1\right)-\left[\gamma+\phi_{\varepsilon}(v)(c-\gamma)\right] u- \\
& -\left[\beta+\phi_{\varepsilon}(v)(b-\beta)-k_{3}^{-} \phi_{\varepsilon}^{\prime}(v)\right] v+O(2), \\
M(u, v, \varepsilon) & =k_{2}^{+}-k_{2}^{-}+\phi_{\varepsilon}(v)\left(k_{2}^{+}-k_{2}^{-}\right)+\left[\beta-a+\phi_{\varepsilon}(v)(b-\beta)-\frac{2 k_{3}^{-} \phi_{\varepsilon}^{\prime}(v)}{\varepsilon}\right] u+ \\
& +\left[\rho-\gamma+\phi_{\varepsilon}(v)(\gamma-c+d-\rho)+\frac{2 \phi_{\varepsilon}^{\prime}(v)\left(k_{2}^{+}-k_{2}^{-}\right)}{\varepsilon}\right] v+O(2), \\
N(u, v, \varepsilon) & =k_{3}^{-}\left(1-\phi_{\varepsilon}(v)\right)+\left[\gamma+\phi_{\varepsilon}(v)(c-\gamma)\right] u+ \\
& +\left[\beta+\phi_{\varepsilon}(v)(b-\beta)-k_{3}^{-} \phi_{\varepsilon}^{\prime}(v)\right] v+O(2) .
\end{aligned}
$$

Fazendo $(u, v)=(0,0)$ na expressão de $L$ dada pela equação 2.48 obtemos:

$$
L(0,0, \varepsilon)=0 \Longleftrightarrow k_{3}^{-}\left(\phi_{\varepsilon}(0)-1\right)=0 \Longleftrightarrow \phi(0)=\phi_{\varepsilon}(0)=1, \quad \varepsilon>0 .
$$

Logo, para $k_{3}^{+}=0, k_{3}^{-} \neq 0$ e $(u, v)=(0,0)$ temos que $\phi(0) \notin(0,1)$ e, por continuidade das funções $L$ e $N$, concluímos que elas não se anulam em uma vizinhança da origem. Portanto, para todo $\varepsilon$ positivo, a superfície regularizada $\mathbf{S}_{\varepsilon}$ não possui pontos umbílicos em uma vizinhança da origem.

Isto completa a demonstração. 
As figuras 2.20 e 2.20 representam pontos de $\boldsymbol{\delta}$-dobras e suas $\varepsilon$-regularizações. A região de regularização foi feita em grande escala para facilitar a visualização das linhas de curvatura principal da superfície regularizada, $\mathbf{S}_{\varepsilon}$, na vizinhança da origem.

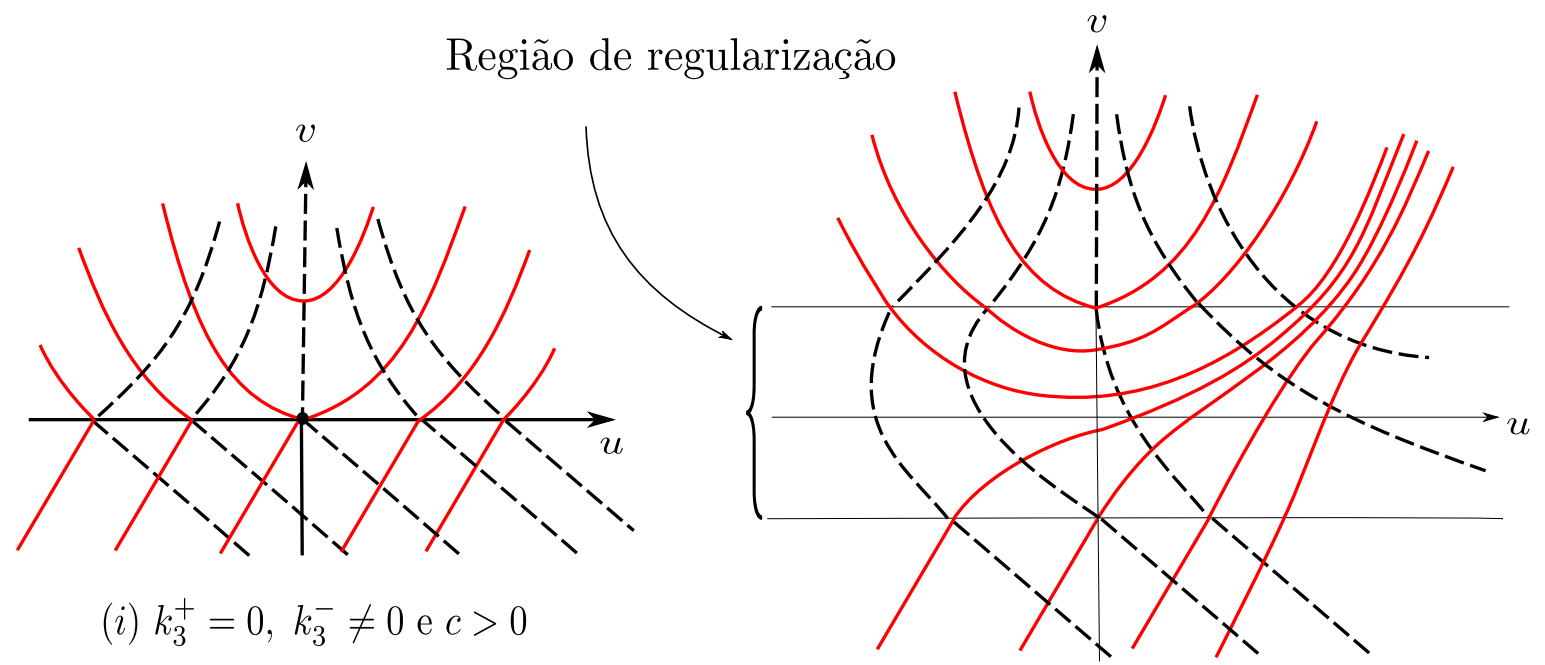

Figura 2.20: $\grave{A}$ esquerda, temos que a origem é um ponto de $\boldsymbol{\delta}$-dobra $\left(k_{3}^{+}=0, k_{3}^{-} \neq 0\right.$ e c $\left.>0\right)$ e, à direita, sua E-regularização.

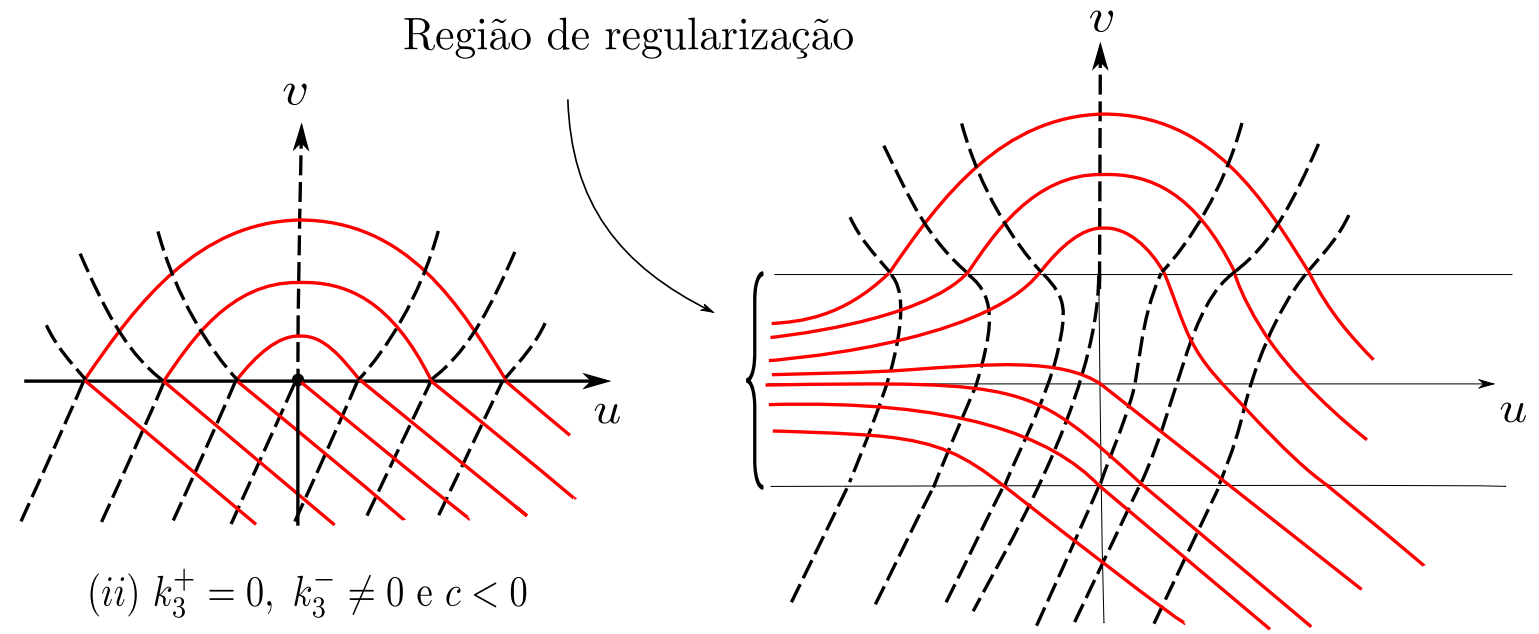

Figura 2.21: $\grave{A}$ esquerda, temos que a origem é um ponto de $\boldsymbol{\delta}$-dobra $\left(k_{3}^{+}=0, k_{3}^{-} \neq 0\right.$ e c $\left.<0\right)$ $e$, à direita, sua $\varepsilon$-regularização. 


\section{Capítulo 3}

\section{Configuração Principal sobre Superfícies Suaves por Partes e Curva de Descontinuidade com Torção}

Sejam $\mathbf{S}^{+}$e $\mathbf{S}^{-}$duas superfícies regulares de classe $C^{\infty}$, compactas, conexas e orientadas com bordo comum $\mathbf{B}$. Considere $\mathbf{S}=\mathbf{S}^{+} \cup \mathbf{B} \cup \mathbf{S}^{-} \subset \mathbb{R}^{3}$ a superfície obtida pela justaposição de $\mathbf{S}^{+}$e $\mathbf{S}^{-}$ao longo do bordo comum $\mathbf{B}$ de tal forma que $\mathbf{S}$ seja de classe $C^{1}$ sobre $\mathbf{B}$. Neste capítulo serão estudadas as linhas de curvatura principal da superfície suave por partes, $\mathbf{S}$, e da superfície suave, $\mathbf{S}_{\varepsilon}$, obtida através da $\varepsilon$-regularização de $\mathbf{S}$ ao longo de $\mathbf{B}, \varepsilon>0$ suficientemente pequeno. No capítulo 2 fizemos um estudo análogo para o caso em que a curva bordo comum $\boldsymbol{\delta}$ é uma curva plana (contida no plano $v=0$ ), agora consideraremos o bordo comum $\mathbf{B}$ de $\mathbf{S}^{+}$e $\mathbf{S}^{-}$como sendo uma curva arbitrária com torção genérica.

Em relação à configuração principal da superfície suave por partes $\mathbf{S}$, o único caso interessante a ser analisado aqui será quando $\delta\left(u_{0}\right)$ for um ponto de tangência quadrática das superfícies $\mathbf{S}^{+}$e $\mathbf{S}^{-}$com o bordo comum B. Veremos na seção 3.3 que dos cruzamentos transversais não emergirão pontos umbílicos e as dobras não são possíveis nesse contexto. $\mathrm{Na}$ seção 3.1 faremos algumas considerações e apresentaremos resultados úteis ao longo do texto. Na seção 3.2 analisaremos os efeitos da regularização dos pontos sobre $\mathbf{B}$ de tangência quadrática. Veremos que, sob certas condições (estabelecidas no teorema 3.2.4), de tais pontos do bordo emergem, sobre $\mathbf{S}_{\varepsilon}$, umbílicos Darbouxianos dos tipos $D_{1}$ e $D_{3}$, para $\varepsilon>0$ suficientemente pequeno. 


\subsection{Configuração Principal da Superfície $\mathbf{S}=\mathbf{S}^{+} \cup \mathbf{B} \cup$ $\mathrm{S}^{-}$: Singularidades Quadráticas e Pontos Umbíli- $\cos$}

Nesta seção será apresentada a formulação do nosso problema para o caso em que o bordo comum B é uma curva qualquer em $\mathbb{R}^{3}$. As proposições 3.1.2 e 3.1 .4 estabelecem as condições para que $\delta\left(u_{0}\right)$ seja um ponto de tangência quadrática tanto para a superfície $\mathbf{S}^{+}$quanto para a superfície $\mathbf{S}^{-}$.

Vamos considerar os pares $\left(\mathbf{S}^{+}, \mathbf{B}\right)$ e $\left(\mathbf{S}^{-}, \mathbf{B}\right)$, sendo $\mathbf{S}^{+}$e $\mathbf{S}^{-}$duas superfícies imersas em $\mathbb{R}^{3}$, regulares de classe $C^{\infty}$, compactas, conexas e orientadas com bordo comum $\mathbf{B}$. Sem perda de generalidade, podemos assumir que $\mathbf{S}^{+}$está contida em $\widehat{\mathbf{S}}^{+}$e $\mathbf{S}^{-}$em $\widehat{\mathbf{S}}^{-}$, sendo $\widehat{\mathbf{S}}^{ \pm}$duas superfícies sem bordo definidas implicitamente pelas funções suaves $f^{+}: \mathbb{R}^{3} \rightarrow \mathbb{R}$ e $f^{-}: \mathbb{R}^{3} \rightarrow \mathbb{R}$ tais que

$$
\begin{aligned}
& \widehat{\mathbf{S}}^{+}=\left\{\mathfrak{p} \in \mathbb{R}^{3}: f^{+}(\mathfrak{p})=0\right\}, \\
& \widehat{\mathbf{S}}^{-}=\left\{\mathfrak{p} \in \mathbb{R}^{3}: f^{-}(\mathfrak{p})=0\right\},
\end{aligned}
$$

e sendo $\mathbf{S}^{ \pm}$e $\mathbf{B}$ dados, em termos de uma função suave $b: \mathbb{R}^{3} \rightarrow \mathbb{R}$, respectivamente por:

$$
\begin{aligned}
& \mathbf{S}^{+}=\left\{\mathfrak{p} \in \widehat{\mathbf{S}}^{+}: b(\mathfrak{p}) \geq 0\right\}, \\
& \mathbf{S}^{-}=\left\{\mathfrak{p} \in \widehat{\mathbf{S}}^{-}: b(\mathfrak{p}) \leq 0\right\}, \\
& \mathbf{B}=\left\{\mathfrak{p} \in \widehat{\mathbf{S}}^{+} \cap \widehat{\mathbf{S}}^{-}: b(\mathfrak{p})=0\right\},
\end{aligned}
$$

com $\nabla f^{ \pm} \neq 0$ sobre $\widehat{\mathbf{S}}^{ \pm}$e $\widehat{T}^{ \pm}=\nabla f^{ \pm} \wedge \nabla b \neq 0$ sobre $\mathbf{B}$.

As funções $f^{ \pm}$são definidas de tais formas que as aplicações normais unitárias positivas das superfícies $\mathbf{S}^{ \pm}$são dadas por:

$$
\mathcal{N}^{+}=\frac{\nabla f^{+}}{\left|\nabla f^{+}\right|} \quad \text { e } \quad \mathcal{N}^{-}=\frac{\nabla f^{-}}{\left|\nabla f^{-}\right|}
$$

Vamos supor que a orientação positiva sobre B é definida pelo campo de vetores unitários tangente $T=\frac{\widehat{T}^{+}}{\left|\widehat{T}^{+}\right|}$. Veja em [5] exemplos de tais superfícies.

Suporemos, ainda, que a superfície $\mathbf{S}=\mathbf{S}^{+} \cup \mathbf{B} \cup \mathbf{S}^{-}$, obtida pela justaposição de $\mathbf{S}^{+}$ e $\mathbf{S}^{-}$ao longo do bordo comum $\mathbf{B}$, está positivamente orientada pela aplicação normal unitária $\mathcal{N}$ de tal forma que $\mathbf{S}$ seja de classe $C^{1}$ sobre $\mathbf{B}$.

A proposição 3.1.1 seguinte, baseada em [6], apresenta uma parametrização para uma componente conexa da curva bordo $\mathbf{B}$. 
Proposição 3.1.1. Considere $\delta:[0, l] \longrightarrow \mathbb{R}^{3}$ uma parametrização regular pelo comprimento de arco de uma componente conexa de $\mathbf{B}$ tal que $\{T, \mathcal{N} \wedge T, \mathcal{N}\}$ é um referencial móvel ao longo de $\delta$, sendo $T(u)$ o vetor tangente unitário à curva $u \longmapsto \delta(u)$. Então as expressões

$$
X^{+}(u, v)=\delta(u)+v(\mathcal{N} \wedge T)(u)+\left[\frac{1}{2} k^{+}(u) v^{2}+\frac{1}{6} A(u) v^{3}+C(u, v) v^{3}\right] \mathcal{N}(u),-\mu<v<\mu
$$

$X^{-}(u, v)=\delta(u)+v(\mathcal{N} \wedge T)(u)+\left[\frac{1}{2} k^{-}(u) v^{2}+\frac{1}{6} D(u) v^{3}+E(u, v) v^{3}\right] \mathcal{N}(u),-\mu<v<\mu$

definem, respectivamente sobre as superfícies $\widehat{\mathbf{S}}^{+}$e $\widehat{\mathbf{S}}^{-}$, uma carta local $(u, v)$ de classe $C^{\infty}$ em uma vizinhança de $\delta$, onde $C(u, 0)=E(u, 0)=0, k^{+}$e $k^{-}$são as curvaturas normais de $\mathbf{S}^{+}$e $\mathbf{S}^{-}$na direção de $B=\mathcal{N} \wedge T$.

Demonstração. Vamos provar que (3.1) define uma carta local $(u, v)$ em uma vizinhança de $\delta$ considerando o par $\left(\mathbf{S}^{+}, \mathbf{B}\right)$. Para o par $\left(\mathbf{S}^{-}, \mathbf{B}\right)$ a demonstração é análoga.

A aplicação

$$
X^{+}(u, v, w)=\delta(u)+v(\mathcal{N} \wedge T)(u)+w \mathcal{N}(u)
$$

é um difeomorfismo local em uma vizinhança do eixo $u$, pois $\left\langle X_{u}^{+} \wedge X_{v}^{+}, X_{u u}^{+}\right\rangle(u, 0,0)=1^{1}$. Portanto, existe uma função $W(u, v)$, de classe $C^{\infty}$, tal que $X^{+}(u, v, W(u, v))$ é uma parametrização de uma vizinhança tubular de $\delta$. Para cada $u, W(u, v)$ é justamente a parametrização da curva

$$
v \longmapsto v(\mathcal{N} \wedge T)(u)+W(u, v) \mathcal{N}(u)
$$

de interseção entre a superfície $\widehat{\mathbf{S}}^{+}$com o plano normal gerado por $\{(\mathcal{N} \wedge T)(u), \mathcal{N}(u)\}$. Esta curva de interseção é tangente à $(\mathcal{N} \wedge T)(u)$ em $v=0$ e $k^{+}(u)$ é a curvatura (plana) da curva no plano gerado por $\{\mathcal{N} \wedge T, \mathcal{N}\}$. Logo,

$$
X^{+}(u, v, W(u, v))=\delta(u)+v(\mathcal{N} \wedge T)(u)+\left[\frac{1}{2} k^{+}(u) v^{2}+\frac{1}{6} A(u) v^{3}+C(u, v) v^{3}\right] \mathcal{N}(u)
$$

onde $A$ é de classe $C^{\infty}$ e $B(u, 0)=0$.

Isto completa a demonstração.

Uma carta similar foi usada em [7] por Gutierrez e Sotomayor para o estudo das linhas de curvatura principal periódicas, em [4] por Garcia e Sotomayor, no estudo das linhas assintóticas na vizinhança de curvas parabólicas e em [5], também por Garcia e Sotomayor,

$$
{ }^{1} X_{u}^{+}=\frac{\partial X^{+}}{\partial u}, X_{v}^{+}=\frac{\partial X^{+}}{\partial v} \text { e } X_{u u}^{+}=\frac{\partial^{2} X^{+}}{\partial u^{2}} .
$$


no estudo de curvas que são bordos de superfícies em $\mathbb{R}^{3}$, como no caso apresentado pela proposição 3.1.1.

O referencial de Darboux $\{T, B=\mathcal{N} \wedge T, \mathcal{N}\}$ ao longo de $\mathbf{B}$ satisfaz o seguinte sistema de equações diferenciais:

$$
\begin{gathered}
T^{\prime}(u)=k_{g}(u) B(u)+k_{n}(u) \mathcal{N}(u), \\
B^{\prime}(u)=-k_{g}(u) T(u)+\tau_{g}(u) \mathcal{N}(u), \\
\mathcal{N}^{\prime}(u)=-k_{n}(u) T(u)-\tau_{g}(u) B(u),
\end{gathered}
$$

onde $k_{n}(u)$ é a curvatura normal, $k_{g}(u)$ é a curvatura geodésica e $\tau_{g}(u)$ é a torção geodésica da curva bordo $\mathbf{B}$.

A proposição 3.1.2, abaixo, estabelece as condições para que as linhas de curvatura principal da superfície $\mathbf{S}^{+}$sejam tangentes quadraticamente ou cruzem transversalmente o bordo B na vizinhança do ponto $\delta\left(u_{0}\right)$.

Proposição 3.1.2. Considere a superfície $\mathbf{S}^{+}$parametrizada, em uma vizinhança do ponto $\left(u_{0}, 0\right)$, por $X^{+}(u, v)=\delta(u)+v B(u)+\left[\frac{1}{2} k^{+}(u) v^{2}+\frac{1}{6} A(u) v^{3}+C(u, v) v^{3}\right] \mathcal{N}(u)$, $-\mu<v<\mu$, sendo $\delta$ uma parametrização pelo comprimento de arco u de uma componente conexa da curva bordo B. Então, as linhas de curvatura principal de $\mathbf{S}^{+}$são transversais ao bordo $\delta$ no ponto $\delta\left(u_{0}\right)$ se, e somente se, $\tau_{g}\left(u_{0}\right) \neq 0$.

Supondo que $\left(k_{n}-k^{+}\right)\left(u_{0}\right)>0$, uma linha de curvatura principal máxima tem contato quadrático com $\delta$ em $\delta\left(u_{0}\right)$ se, e somente se, $\tau_{g}\left(u_{0}\right)=0$ e $\tau_{g}^{\prime}\left(u_{0}\right) \neq 0$. Este contato é interno (respectivamente externo) se $\tau_{g}^{\prime}\left(u_{0}\right)>0$ (respectivamente $\tau_{g}^{\prime}\left(u_{0}\right)<0$ ). Veja figura 3.1 .
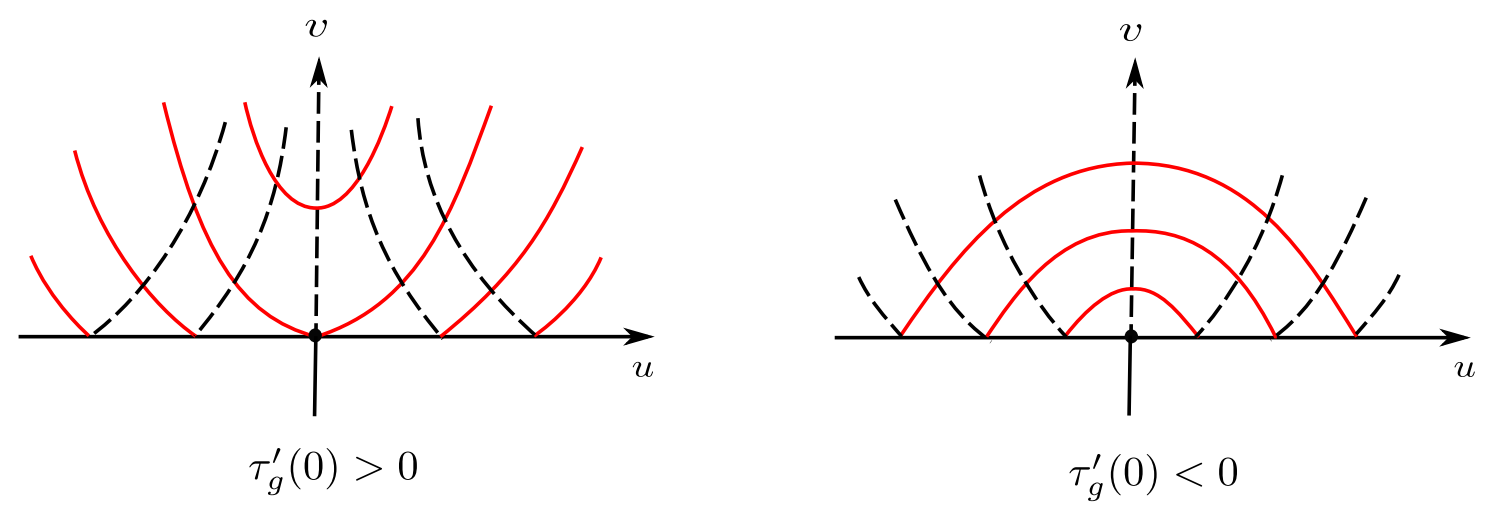

Figura 3.1: Configuração principal de $\mathbf{S}^{+}$em uma vizinhança de $\delta\left(u_{0}\right)$ : tangência interna para $\tau_{g}^{\prime}\left(u_{0}\right)>0$ e externa para $\tau_{g}^{\prime}\left(u_{0}\right)<0$, considerando $u_{0}=0$. Supondo $\left(k_{n}-k^{+}\right)\left(u_{0}\right)>0$, temos que as linhas tracejadas (respectivamente contínuas) ilustram as linhas de curvatura principal mínima (respectivamente máxima). 
Demonstração. Usando a parametrização da superfície $\mathbf{S}^{+}$da proposição 3.1, a qual é dada por:

$$
X^{+}(u, v)=\delta(u)+v B(u)+\left[\frac{1}{2} k^{+}(u) v^{2}+\frac{1}{6} A(u) v^{3}+C(u, v) v^{3}\right] \mathcal{N}(u), \quad-\mu<v<\mu
$$

e o referencial de Darboux ao longo de $\mathbf{B}$ dado pelas equações (3.3), temos que os coeficientes da Primeira e Segunda Formas Fundamentais em $(u, 0)$ são:

$$
\begin{aligned}
E(u, 0) & =1, & F(u, 0) & =0, & G(u, 0) & =1, \\
e(u, 0) & =k_{n}(u), & f(u, 0) & =\tau_{g}(u), & g(u, 0) & =k^{+}(u) .
\end{aligned}
$$

As direções principais de $\mathbf{S}^{+}$em $(u, 0)$ satisfazem:

$$
-\tau_{g}(u) d v^{2}+\left(k^{+}-k_{n}\right)(u) d u d v+\tau_{g}(u) d u^{2}=0 .
$$

Portanto, o vetor $T(u)$ é uma direção principal de (3.5) se, e somente se, $\tau_{g}(u)=0$.

Próximo do ponto $\left(u_{0}, 0\right)$ a equação diferencial das linhas de curvatura de $\mathbf{S}^{+}$é dada por

$$
L(u, v) d v^{2}+M(u, v) d u d v+N(u, v) d u^{2}=0,
$$

sendo

$$
\begin{aligned}
& L(u, v)=(F g-G f)(u, v)=-\tau_{g}(u)-\left(k^{+}\right)^{\prime}(u) v+O\left(v^{2}\right), \\
& M(u, v)=(E g-G e)(u, v)=k^{+}(u)-k^{-}(u)+ \\
&+\left[2 k_{g}(u) k_{n}(u)-\tau_{g}^{\prime}(u)-2 k_{g}(u) k^{+}(u)\right] v+O\left(v^{2}\right), \\
& N(u, v)=(E f-F e)(u, v)=\tau_{g}(u)+\left[\left(k^{+}\right)^{\prime}(u)-2 k_{g}(u) \tau_{g}(u)\right] v+O\left(v^{2}\right) .
\end{aligned}
$$

Usando o "Método dos Coeficientes a Determinar" temos que na vizinhança do ponto de tangência $\left(u_{0}, 0\right)$, as linhas de curvatura principal máxima são soluções de (3.6) e descritas por

$$
v(u)=\frac{1}{2} \frac{\tau_{g}^{\prime}\left(u_{0}\right)}{\left(k_{n}-k^{+}\right)\left(u_{0}\right)}\left(u-u_{0}\right)^{2}+\cdots
$$

Isto completa a demonstração.

Observação 3.1.3. Na proposição 3.1.2 mudamos a orientação de $\mathbf{S}^{+}$se considerarmos $\left(k^{+}-k_{n}\right)\left(u_{0}\right)>0$ logo, supondo que $\tau_{g}\left(u_{0}\right)=0$, uma linha de curvatura principal mínima terá contato quadrático com $\delta$ em $\delta\left(u_{0}\right)$ interno (respectivamente externo) se $\tau_{g}^{\prime}\left(u_{0}\right)<0$ (respectivamente $\tau_{g}^{\prime}\left(u_{0}\right)>0$ ). 
A proposição seguinte é a análoga da proposição 3.1 .2 para a superfície $\mathbf{S}^{-}$.

Proposição 3.1.4. Considere a superfície $\mathbf{S}^{-}$parametrizada, em uma vizinhança do ponto $\left(u_{0}, 0\right)$, por $X^{-}(u, v)=\delta(u)+v B(u)+\left[\frac{1}{2} k^{-}(u) v^{2}+\frac{1}{6} D(u) v^{3}+E(u, v) v^{3}\right] \mathcal{N}(u)$, $-\mu<v<\mu$, sendo $\delta$ uma parametrização pelo comprimento de arco u de uma componente conexa da curva bordo $\mathbf{B}$. Então, as linhas de curvatura principal de $\mathbf{S}^{-}$são transversais ao bordo $\delta$ no ponto $\delta\left(u_{0}\right)$ se, e somente se, $\tau_{g}\left(u_{0}\right) \neq 0$.

Supondo que $\left(k_{n}-k^{-}\right)\left(u_{0}\right)<0$, uma linha de curvatura principal minima tem contato quadrático com $\delta$ em $\delta\left(u_{0}\right)$ se, e somente se, $\tau_{g}\left(u_{0}\right)=0$ e $\tau_{g}^{\prime}\left(u_{0}\right) \neq 0$. Este contato é interno (respectivamente externo) se $\tau_{g}^{\prime}\left(u_{0}\right)>0$ (respectivamente $\tau_{g}^{\prime}\left(u_{0}\right)<0$ ). Veja figura 3.2.
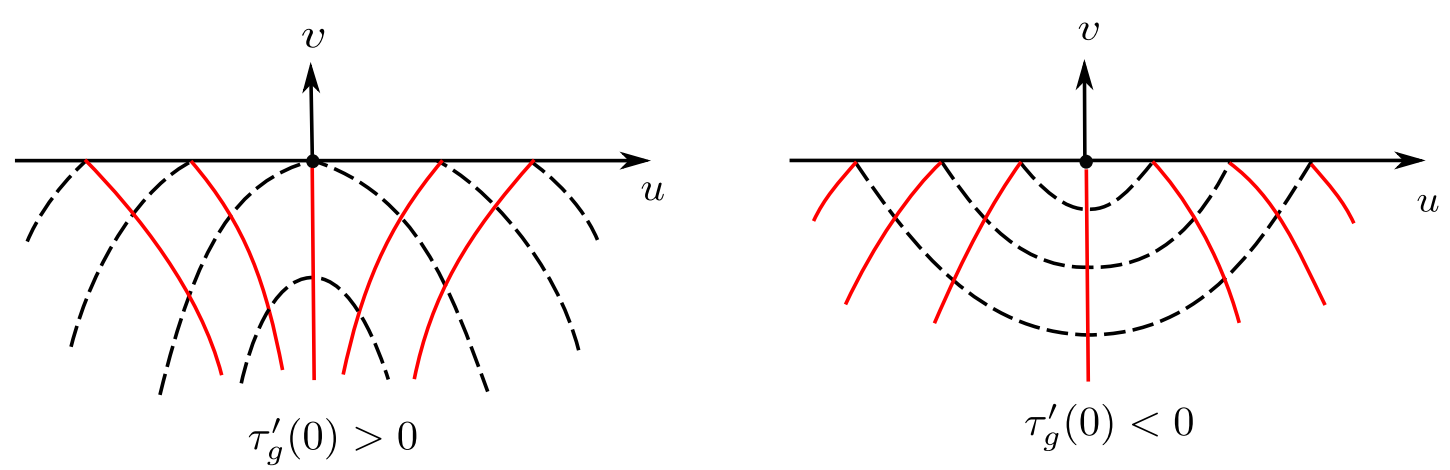

Figura 3.2: Configuração principal de $\mathbf{S}^{-}$em uma vizinhança da origem: tangência interna para $\tau_{g}^{\prime}\left(u_{0}\right)>0$ e externa para $\tau_{g}^{\prime}\left(u_{0}\right)<0$, considerando $u_{0}=0$. Supondo $\left(k_{n}-k^{-}\right)\left(u_{0}\right)<0$, temos que as linhas tracejadas (respectivamente contínuas) ilustram as linhas de curvatura principal mínimas (respectivamente máximas).

Demonstração. Usando a parametrização da superfície $\mathbf{S}^{-}$da proposição 3.1, a qual é dada por:

$$
X^{-}(u, v)=\delta(u)+v B(u)+\left[\frac{1}{2} k^{-}(u) v^{2}+\frac{1}{6} D(u) v^{3}+E(u, v) v^{3}\right] \mathcal{N}(u), \quad-\mu<v<\mu
$$

e o referencial de Darboux ao longo de $\mathbf{B}$ dado pelas equações 3.3 temos que os coeficientes da Primeira e Segunda Formas Fundamentais em $(u, 0)$ são:

$$
\begin{aligned}
E(u, 0) & =1, & F(u, 0) & =0, & G(u, 0) & =1, \\
e(u, 0) & =k_{n}(u), & f(u, 0) & =\tau_{g}(u), & g(u, 0) & =k^{-}(u) .
\end{aligned}
$$

A demonstração segue análoga à da proposição 3.1 .2 sendo que próximo da tangência, as linhas de curvatura principal mínima são descritas por

$$
v(u)=\frac{1}{2} \frac{\tau_{g}^{\prime}\left(u_{0}\right)}{\left(k_{n}-k^{-}\right)\left(u_{0}\right)}\left(u-u_{0}\right)^{2}+\cdots .
$$


Isto completa a demonstração.

Observação 3.1.5. Na proposição 3.1.4 mudamos a orientação se considerarmos $\left(k_{n}-k^{-}\right)\left(u_{0}\right)>0$. Logo, supondo que $\tau_{g}\left(u_{0}\right)=0$, uma linha de curvatura principal máxima terá contato quadrático com $\delta$ em $\delta\left(u_{0}\right)$ interno (respectivamente externo) se $\tau_{g}^{\prime}\left(u_{0}\right)<0$ (respectivamente $\tau_{g}^{\prime}\left(u_{0}\right)>0$ ).

A curva parametrizada por $\delta(u)=X^{+}(u, 0)=X^{-}(u, 0)$ é o bordo comum das superfícies $\mathbf{S}^{+}$e $\mathbf{S}^{-}$(faça $v=0$ em $X^{+}$da proposição 3.1 .2 e em $X^{-}$da proposição 3.1.4). Considere $\mathbf{S}=\mathbf{S}^{+} \cup \mathbf{B} \cup \mathbf{S}^{-}$como sendo a superfície sem bordo de classe $C^{1}$ que foi obtida pela justaposição de $\mathbf{S}^{+}$e $\mathbf{S}^{-}$ao longo de $\mathbf{B}$. Observe que $\mathbf{S}$ é suave no complementar da curva bordo comum $\mathbf{B}$ e de classe $\mathbf{C}^{1}$ ao longo desta curva.

Supondo que $\tau_{g}\left(u_{0}\right)=0$, as folheações principais das superfícies $\mathbf{S}^{+}$e $\mathbf{S}^{-}$, em uma vizinhança do ponto de tangência $\delta\left(u_{0}\right)$, foram descritas nas proposições 3.1.2 e 3.1.4. respectivamente. Estas considerações permitem-nos adotar a seguinte definição.

Definição 3.1.6. Seja $\mathbf{S}=\mathbf{S}^{+} \cup \mathbf{B} \cup \mathbf{S}^{-}$a superfície obtida pela justaposição de $\mathbf{S}^{+}$e $\mathbf{S}^{-}$ao longo de $\mathbf{B}$. A configuração principal de $\mathbf{S}=\mathbf{S}^{+} \cup \mathbf{B} \cup \mathbf{S}^{-}$, isto é, suas singularidades e linhas das folheações principais são como segue:

(I) As linhas principais minimas de $\mathbf{S}$, fora das tangências, são as linhas principais mínimas da superfície $\mathbf{S}^{+}$justapostas com as linhas principais minimas da superfície $\mathrm{S}^{-}$.

(II) As linhas principais máximas de $\mathbf{S}$, fora das tangências, são as linhas principais máximas da superfície $\mathbf{S}^{+}$justapostas com as linhas principais máximas da superfície $\mathbf{S}^{-}$.

(III) As singularidades de $\mathbf{S}$ são os pontos umbílicos de $\mathbf{S}^{+}$e $\mathbf{S}^{-}$e os pontos de $\mathbf{B}$ de tangência com alguma das linhas de curvatura principal destas superfícies.

As figuras 3.3 e 3.4 abaixo ilustram as possiveis configurações topológicas de $\mathbf{S}::^{2}$

\footnotetext{
${ }^{2}$ Para esboçar as linhas de curvatura de $\mathbf{S}$ basta analisar o sinal de $\tau_{g}^{\prime}\left(u_{0}\right)$ nas proposições 3.1 .2 e 3.1 .4 observando a definição 3.1 .6 acima e as possibilidades $k^{+}\left(u_{0}\right)<k_{n}\left(u_{0}\right)<k^{-}\left(u_{0}\right)$ e $\left(k_{n}\left(u_{0}\right)<k^{+}\left(u_{0}\right)\right.$ e $\left.k_{n}\left(u_{0}\right)<k^{-}\left(u_{0}\right)\right)$.
} 

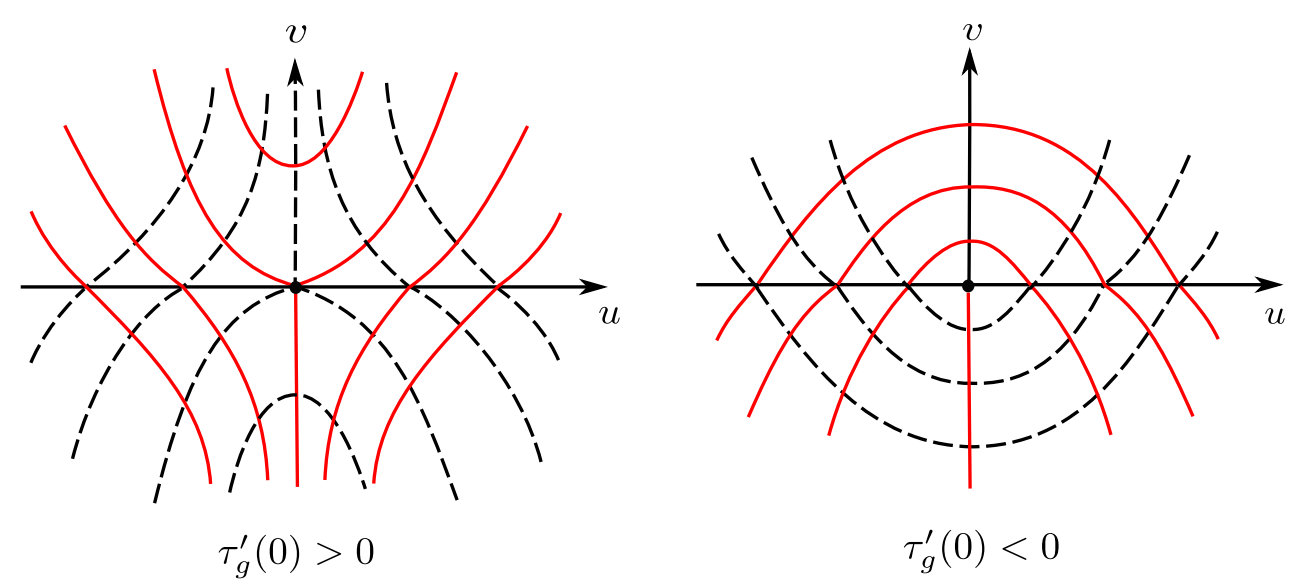

Figura 3.3: Configuração principal suave por partes de $\mathbf{S}=\mathbf{S}^{+} \cup \mathbf{B} \cup \mathbf{S}^{-}$em uma vizinhança da origem considerando $u_{0}=0$ e $k^{+}\left(u_{0}\right)<k_{n}\left(u_{0}\right)<k^{-}\left(u_{0}\right)$. Linhas tracejadas (respectivamente contínuas) ilustram as linhas de curvatura principal mínimas (respectivamente máximas). Dois casos: $\left.\tau_{g}^{\prime}\left(u_{0}\right)\right)>0$, à esquerda e $\tau_{g}^{\prime}\left(u_{0}\right)<0$, à direita.
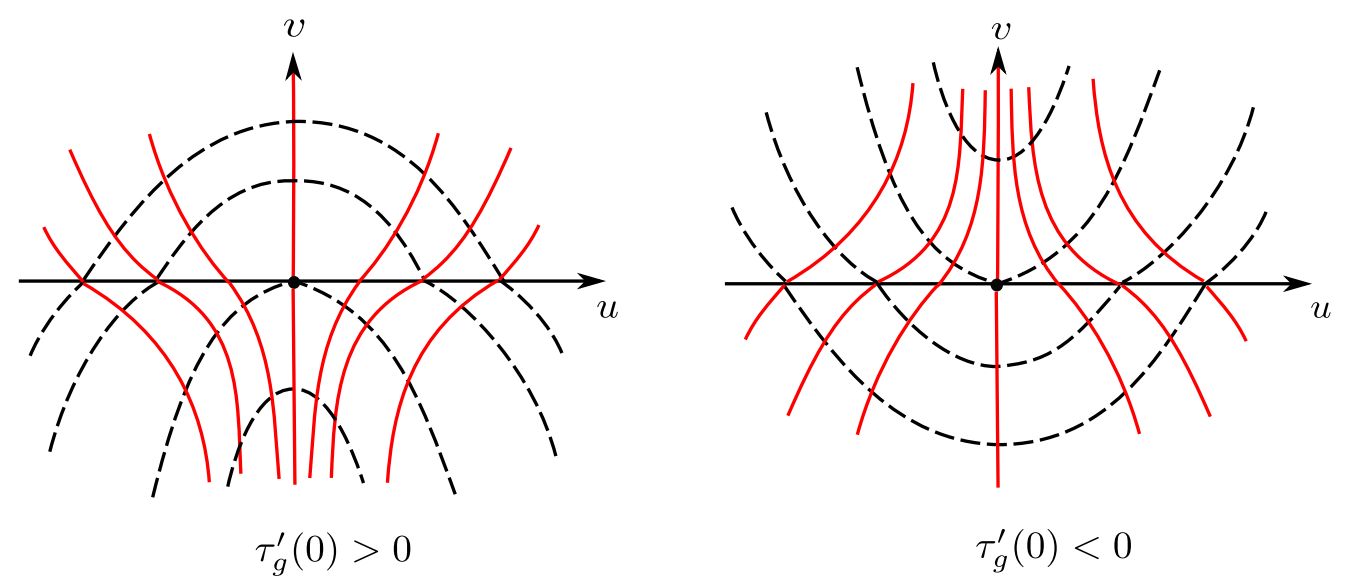

Figura 3.4: Configuração principal suave por partes de $\mathbf{S}=\mathbf{S}^{+} \cup \mathbf{B} \cup \mathbf{S}^{-}$em uma vizinhança da origem considerando $u_{0}=0$ e $k_{n}\left(u_{0}\right)<k^{+}\left(u_{0}\right)$ e $k_{n}\left(u_{0}\right)<k^{-}\left(u_{0}\right)$. Linhas tracejadas (respectivamente contínuas) ilustram as linhas de curvatura principal minimas (respectivamente máximas). Dois casos: $\tau_{g}^{\prime}\left(u_{0}\right)>0$, à esquerda e $\tau_{g}^{\prime}\left(u_{0}\right)<0$, à direita.

\subsection{Regularização da Superfície S}

Nesta seção analisaremos os efeitos da regularização sobre os pontos da curva bordo comum $\mathbf{B}$ de tangência quadrática das linhas de curvatura principal das superfícies $\mathbf{S}^{+}$ e $\mathbf{S}^{-}$com B. Veremos que, sob certas condições (estabelecidas no teorema 3.2), após o processo de regularização, a configuração principal de $\mathbf{S}$ será equivalente à configuração principal na vizinhança dos pontos umbílicos Darbouxianos dos tipos $D_{1}$ ou $D_{3}$.

Vamos adotar as seguintes definições:

Definição 3.2.1. Seja $\phi: \mathbb{R} \longrightarrow \mathbb{R}$ uma função de transição tal que $\phi^{(n)}(0)=$ $\phi^{(n)}(\mu)=0$, para todo $n \in \mathbb{N}, \phi^{\prime}(t)<0$, para $t \in(0, \mu), \phi(t)=0$, se $t \geq \mu$ e $\phi(t)=1$, se $t \leq 0$ (no apêndice 4.4 apresentamos um exemplo de uma tal função $\phi$ ). 
Definição 3.2.2. Sejam $\phi$ uma função de transição e $\mathbf{S}=\mathbf{S}^{+} \cup \mathbf{B} \cup \mathbf{S}^{-}$ uma superfície sem bordo em $\mathbb{R}^{3}$, sendo $\mathbf{S}^{+}, \mathbf{S}^{-}$e $\mathbf{B}$ definidas anteriormente. Para $\varepsilon>0$, a $\phi_{\varepsilon}$-regularização de $\mathbf{S}$, denotada por $\mathbf{S}_{\varepsilon}$, é (não unicamente) definida por:

$$
X(u, v, \varepsilon)=\phi\left(\frac{v}{\varepsilon^{2}}\right) X^{-}(u, v)+\left[1-\phi\left(\frac{v}{\varepsilon^{2}}\right)\right] X^{+}(u, v), \quad-\varepsilon^{2} \mu<v<\varepsilon^{2} \mu,
$$

onde $X^{+}, X^{-}$são as parametrizações das superfícies $\mathbf{S}^{+}$e $\mathbf{S}^{-}$dadas, respectivamente, pelas expressões (3.1) e (3.2) da proposição 3.1.1, as quais serão reescritas abaixo:

$$
\begin{aligned}
& X^{+}(u, v)=\delta(u)+v(\mathcal{N} \wedge T)(u)+\left[\frac{1}{2} k^{+}(u) v^{2}+\frac{1}{6} A(u) v^{3}+C(u, v) v^{3}\right] \mathcal{N}(u), 0 \leq v<\mu \\
& X^{-}(u, v)=\delta(u)+v(\mathcal{N} \wedge T)(u)+\left[\frac{1}{2} k^{-}(u) v^{2}+\frac{1}{6} D(u) v^{3}+E(u, v) v^{3}\right] \mathcal{N}(u),-\mu<v \leq 0
\end{aligned}
$$

lembrando que $\{T, \mathcal{N} \wedge T, \mathcal{N}\}$ é um referencial positivo de $\mathbb{R}^{3}$.

Assim, a equação 3.9 torna-se:

$$
\begin{aligned}
X(u, v, \varepsilon) & =\delta(u)+v(\mathcal{N} \wedge T)(u)+\left\{\left[\frac{1}{2}\left(1-\phi\left(\frac{v}{\varepsilon^{2}}\right)\right) k^{+}(u)+\frac{1}{2} \phi\left(\frac{v}{\varepsilon^{2}}\right) k^{-}(u)\right] v^{2}+\right. \\
& \left.+\left[\left(1-\phi\left(\frac{v}{\varepsilon^{2}}\right)\right)\left(\frac{A(u)}{2}+C(u, v)\right)+\phi\left(\frac{v}{\varepsilon^{2}}\right)\left(\frac{D(u)}{2}+E(u, v)\right)\right] v^{3}\right\} \mathcal{N}(u),
\end{aligned}
$$

$-\varepsilon^{2} \mu<v<\varepsilon^{2} \mu, \quad \operatorname{com} C(u, 0)=E(u, 0)=0$.

Façamos o reescalonamento $v=\varepsilon^{2} w$ na expressão (3.10). Logo, na carta $(u, w)$, a parametrização de $\mathbf{S}_{\varepsilon}$ é dada por:

$$
\begin{aligned}
X(u, w, \varepsilon) & =\delta(u)+\varepsilon^{2} w(\mathcal{N} \wedge T)(u)+\left\{\varepsilon^{4}\left[\frac{1}{2}(1-\phi(w)) k^{+}(u)+\frac{1}{2} \phi(w) k^{-}(u)\right] w^{2}+\right. \\
& +\varepsilon^{6}\left[(1-\phi(w))\left(\frac{A(u)}{2}+\varepsilon^{2} C(u, w)\right)+\right. \\
& \left.\left.+\phi(w)\left(\frac{D(u)}{2}+\varepsilon^{2} E(u, w)\right)\right] w^{3}\right\} \mathcal{N}(u), \quad-\mu<w<\mu
\end{aligned}
$$

$\operatorname{com} C(u, 0)=E(u, 0)=0$.

O nosso objetivo é estudar os pontos umbílicos Darbouxianos sobre a superfície regularizada $\mathbf{S}_{\varepsilon}, \varepsilon>0$. Para isso vamos determinar os coeficientes da Primeira e Segunda Formas Fundamentais de $X_{\varepsilon}(u, w)=X(u, w, \varepsilon)$ e obter a equação diferencial das linhas de curvatura na carta $(u, w)$, a qual é dada por:

$$
L(u, w, \varepsilon) d w^{2}+M(u, w, \varepsilon) d u d w+N(u, w, \varepsilon) d u^{2}=0
$$


sendo $L=F g-G f, \quad M=E g-G e$ e $N=E f-F e$.

Abaixo, omitiremos a variável $u$ das funções $k^{+}(u), k^{-}(u), k_{n}(u), k_{g}(u)$ e $\tau_{g}(u)$ e a variável $w$ da função $\phi(w)$ a fim de simplificar a notação.

Da equação (3.11) temos, na carta $(u, w)$, que:

$$
\begin{aligned}
\frac{\partial X_{\varepsilon}}{\partial u} & =\left\{1-\varepsilon^{2} k_{g} w-\frac{1}{2} \varepsilon^{4} k_{n} w^{2}\left[(1-\phi) k^{+}+\phi k^{-}+\varepsilon w(\cdots)\right]\right\} T(u)+ \\
& +\left\{-\frac{1}{2} \varepsilon^{4} \tau_{g} w^{2}\left[(1-\phi) k^{+}+\phi k^{-}+\varepsilon^{2} w(\cdots)\right]\right\} B(u)+ \\
& +\left\{\varepsilon^{2} \tau_{g} w+\frac{1}{2} \varepsilon^{4} w^{2}\left[(1-\phi)\left(k^{+}\right)^{\prime}+\phi\left(k^{-}\right)^{\prime}+\varepsilon w(\cdots)\right]\right\} \mathcal{N}(u) \\
\frac{\partial X_{\varepsilon}}{\partial w} & =\varepsilon^{2} B(u)+\left\{\varepsilon^{4} w\left[(1-\phi) k^{+}+\phi k^{-}-\frac{1}{2} w \phi^{\prime}\left(k^{+}-k^{-}\right)+\varepsilon^{2} w(\cdots)\right]\right\} \mathcal{N}(u) .
\end{aligned}
$$

O vetor normal (não unitário) a $\mathbf{S}_{\varepsilon}$ é dado por:

$$
\mathcal{N}_{\varepsilon}(u, w)=\left(\left(X_{\varepsilon}\right)_{u} \wedge\left(X_{\varepsilon}\right)_{w}\right)(u, w)=n_{1} T(u)+n_{2} B(u)+n_{3} \mathcal{N}(u),
$$

onde

$$
\begin{aligned}
& n_{1}=-\varepsilon^{4} \tau_{g} w-\frac{1}{2} \varepsilon^{6} w^{2}\left[(1-\phi)\left(k^{+}\right)^{\prime}+\phi\left(k^{-}\right)^{\prime}+\varepsilon^{2} w(\cdots)\right], \\
& n_{2}=-\varepsilon^{4} w\left[k^{+}\left(1-\phi-\frac{1}{2} w \phi^{\prime}\right)+k^{-}\left(\phi+\frac{1}{2} w \phi^{\prime}\right)\right]+\varepsilon^{6} w^{2}(\cdots), \\
& n_{3}=\varepsilon^{2}-\varepsilon^{4} k_{g} w-\frac{1}{2} \varepsilon^{6} k_{n} w^{2}\left[(1-\phi) k^{+}+\phi k^{-}+\varepsilon^{2} w(\cdots)\right] .
\end{aligned}
$$

Os coeficientes da Primeira Forma Fundamental de $X_{\varepsilon}$, expressos na carta $(u, w)$, são:

$$
\begin{aligned}
E(u, w, \varepsilon) & =\left\langle\frac{\partial X_{\varepsilon}}{\partial u}, \frac{\partial X_{\varepsilon}}{\partial u}\right\rangle=1-2 \varepsilon^{2} k_{g} w+ \\
& +\varepsilon^{4} w^{2}\left[-k_{n} k^{+}(1-\phi)-k_{n} k^{-} \phi+\tau_{g}^{2}+k_{g}^{2}\right]+\varepsilon^{6} w^{3}(\cdots), \\
F(u, w, \varepsilon) & =\left\langle\frac{\partial X_{\varepsilon}}{\partial u}, \frac{\partial X_{\varepsilon}}{\partial w}\right\rangle=\frac{1}{2} \varepsilon^{6} w^{2}\left[\tau_{g} k^{-}\left(\phi+w \phi^{\prime}\right)+\right. \\
& \left.+\tau_{g} k^{+}\left(1-\phi-w \phi^{\prime}\right)\right]+\varepsilon^{8} w^{2}(\cdots),
\end{aligned}
$$




$$
\begin{aligned}
G(u, w, \varepsilon) & =\left\langle\frac{\partial X_{\varepsilon}}{\partial w}, \frac{\partial X_{\varepsilon}}{\partial w}\right\rangle=\varepsilon^{4}+\frac{1}{4} \varepsilon^{8} w^{2}\left[k^{-}\left(2 \phi+w \phi^{\prime}\right)+\right. \\
& \left.+k^{+}\left(2-2 \phi-w \phi^{\prime}\right)\right]^{2}+\varepsilon^{8} w^{3}(\cdots)
\end{aligned}
$$

Os coeficientes da Segunda Forma Fundamental de $X_{\varepsilon}$, expressos na carta $(u, w)$, são dados por $\frac{e}{\left|\mathcal{N}_{\varepsilon}\right|}, \frac{f}{\left|\mathcal{N}_{\varepsilon}\right|}$ e $\frac{g}{\left|\mathcal{N}_{\varepsilon}\right|}$, onde $\mathcal{N}_{\varepsilon}$ é o vetor normal definido em 3.12 e

$$
\begin{aligned}
e(u, w, \varepsilon) & =\left\langle\frac{\partial^{2} X_{\varepsilon}}{\partial u^{2}}, \mathcal{N}_{\varepsilon}\right\rangle(u, w, \varepsilon)=\varepsilon^{2} k_{n}+\varepsilon^{4} w\left[\tau_{g}^{\prime}-2 k_{g} k_{n}+\right. \\
& \left.+k_{g} k^{+}\left(-1+\phi+\frac{1}{2} w \phi^{\prime}\right)+k_{g} k^{+}\left(-\phi-\frac{1}{2} w \phi^{\prime}\right)\right]+\varepsilon^{6} w^{2}(\cdots) \\
f(u, w, \varepsilon) & =\left\langle\frac{\partial^{2} X_{\varepsilon}}{\partial u \partial w}, \mathcal{N}_{\varepsilon}\right\rangle(u, w, \varepsilon)=\varepsilon^{4} \tau_{g}+\varepsilon^{6} w\left[\left(k^{+}\right)^{\prime}\left(1-\phi-\frac{1}{2} w \phi^{\prime}\right)+\right. \\
& \left.+\left(k^{-}\right)^{\prime}\left(\phi+\frac{1}{2} w \phi^{\prime}\right)\right]+\varepsilon^{8} w^{2}(\cdots) \\
g(u, w, \varepsilon) & =\left\langle\frac{\partial^{2} X_{\varepsilon}}{\partial w^{2}}, \mathcal{N}_{\varepsilon}\right\rangle(u, w, \varepsilon)=\varepsilon^{6}\left[k^{+}\left(1-\phi-2 w \phi^{\prime}-\frac{1}{2} w^{2} \phi^{\prime \prime}\right)+\right. \\
& \left.+k^{-}\left(\phi+2 w \phi^{\prime}+\frac{1}{2} w^{2} \phi^{\prime \prime}\right)\right]+\varepsilon^{8} w(\cdots)
\end{aligned}
$$

Sendo $L=F g-G f, \quad M=E g-G e$ e $N=E f-F e$, dos cálculos acima podemos concluir que:

$$
\begin{aligned}
L(u, w, \varepsilon) & =-\varepsilon^{8} \tau_{g}(u)+\varepsilon^{10} w\left\{\left(k^{-}(u)\right)^{\prime}\left[-\phi(w)-\frac{1}{2} w \phi^{\prime}(w)\right]+\right. \\
& \left.+\left(k^{+}(u)\right)^{\prime}\left[-1+\phi(w)+\frac{1}{2} w \phi^{\prime}(w)\right]\right\}+\varepsilon^{12} w^{2}(\cdots), \\
M(u, w, \varepsilon) & =\varepsilon^{6}\left\{-k_{n}(u)+k^{-}(u)\left[\phi(w)+2 w \phi^{\prime}(w)+\frac{1}{2} w^{2} \phi^{\prime \prime}(w)\right]+\right. \\
& \left.+k^{+}(u)\left[1-\phi(w)-2 w \phi^{\prime}(w)-\frac{1}{2} w^{2} \phi^{\prime \prime}(w)\right]\right\}+\varepsilon^{8} w(\cdots), \\
N(u, w, \varepsilon) & =\varepsilon^{4} \tau_{g}(u)+\varepsilon^{6} w\left\{\left(k^{+}(u)\right)^{\prime}\left[1-\phi(w)-\frac{1}{2} w \phi^{\prime}(w)\right]+\right. \\
& \left.+\left(k^{-}(u)\right)^{\prime}\left[\phi(w)+\frac{1}{2} w \phi^{\prime}(w)\right]-2 k_{g}(u) \tau_{g}(u)\right\}+\varepsilon^{8} w^{2}(\cdots) .
\end{aligned}
$$

Sabemos das proposições 3.1.2 e 3.1.4 da seção 3.1 que as linhas de curvatura principal 
das superfícies $\mathbf{S}^{+}$e $\mathbf{S}^{-}$tem contato quadrático com a curva bordo comum $\mathbf{B}$ em $\delta\left(u_{0}\right)$ se, e somente se, $\tau_{g}\left(u_{0}\right)=0$ e $\tau_{g}^{\prime}\left(u_{0}\right) \neq 0$.

O lema 3.2.3, seguinte, apresenta as condições para tenhamos pontos umbílicos sobre a superfície regularizada $\mathbf{S}_{\varepsilon}, \varepsilon>0$ suficientemente pequeno.

Lema 3.2.3. Seja $\delta\left(u_{0}\right)$ um ponto de tangência quadrática das superfícies $\mathbf{S}^{+}$e $\mathbf{S}^{-}$tal que $\tau_{g}\left(u_{0}\right)=0$ e $\tau_{g}^{\prime}\left(u_{0}\right) \neq 0$. Se $k^{+}\left(u_{0}\right)<k_{n}\left(u_{0}\right)<k^{-}\left(u_{0}\right)$, então o conjunto

$$
U_{\varepsilon}=\{(u, v, \varepsilon): M(u, v, \varepsilon)=N(u, v, \varepsilon)=0\}
$$

é uma curva contínua. A curva de pontos umbilicos $X\left(U_{\varepsilon}\right)$ é tangente ao vetor normal $N\left(\delta\left(u_{0}\right)\right)$ e é transversal à superfície regularizada $\mathbf{S}_{\varepsilon}$ em uma vizinhança deste ponto de tangência.

Demonstração. Suponhamos, sem perda de generalidade, que $u_{0}=0$.

Nas coordenadas $(u, w, \varepsilon)$ (sendo $\left.v=\varepsilon^{2} w\right)$ temos que a expressão de $N(u, w, \varepsilon)$ está definida em (3.15).

Vamos resolver $N(u, w, \varepsilon)=0$ na vizinhança de $(u, w, \varepsilon)=(0,0, \varepsilon)$.

Como $\varepsilon>0$, segue que $N(u, w, \varepsilon)=0$ se, e somente se, $\tilde{N}=\frac{N}{\varepsilon^{4}}=0$.

Observe que $\tilde{N}(0,0, \varepsilon)=\tau_{g}(0)=0$ e $\tilde{N}_{u}(0,0, \varepsilon)=\tau_{g}^{\prime}(0) \neq 0$, por hipótese. Portanto, pelo Teorema da Função Implícita, exite uma função suave $u=u(w, \varepsilon)$ em uma vizinhança de $(0,0, \varepsilon)$, tal que $\tilde{N}(u(w, \varepsilon), w, \varepsilon)=0, u(w, \varepsilon)=\varepsilon^{2} \xi(w, \varepsilon)$ e $\xi(0, \varepsilon)=0$.

Vamos resolver agora $\tilde{M}(w, \varepsilon)=\tilde{M}(u(w, \varepsilon), w, \varepsilon)$, sendo $M$ definido na expressão 3.15 e $\tilde{M}=\frac{M}{\varepsilon^{6}}$.

Como $\phi(0)=1$ e $\phi(\mu)=0$, pelas propriedades da função de transição $\phi$ (veja um exemplo de uma $\phi$ com estas propriedades no apêndice 4.4 do capítulo 4), podemos concluir que

$$
\tilde{M}(0,0)=\left(k^{-}-k_{n}\right)(0)>0, \quad \tilde{M}(\mu, 0)=\left(k^{+}-k_{n}\right)(0)<0
$$

por hipótese. Portanto, pelo Teorema do Valor Intermediário, existe $w_{0} \in(0, \mu)$ tal que $\tilde{M}\left(w_{0}, 0\right)=0$. Defina a seguinte função

$$
H(w)=\phi(w)+2 w \phi^{\prime}(w)+\frac{1}{2} w^{2} \phi^{\prime \prime}(w) .
$$

Assim, devemos resolver a seguinte equação

$$
\tilde{M}(w, \varepsilon)=-k_{n}(0)+k^{+}(0)+\left[k^{-}(0)-k^{+}(0)\right] H(w)+\varepsilon^{2} w(\cdots)=0 .
$$

Podemos considerar a função de transição $\phi$ tal que $H$ seja uma função decrescente 
para $w$ próximo de zero, e portanto, inversível. Logo, da equação (3.16) concluímos que $w=\varphi(\varepsilon)$.

Retornando às coordenadas originais $(u, v, \varepsilon)$, temos que $u=\varepsilon^{2} \xi(w, \varepsilon)=\varepsilon^{2} \xi(\varphi(\varepsilon), \varepsilon)$, $v=\varepsilon^{2} w=\varepsilon^{2} \varphi(\varepsilon)$ e a curva de pontos umbílicos (no parâmetro $\varepsilon>0$ ) é dada por

$$
U_{\varepsilon}=\left(\varepsilon^{2} \xi(\varphi(\varepsilon), \varepsilon), \varepsilon^{2} \varphi(\varepsilon), \varepsilon\right)
$$

Cálculos diretos mostram que $U_{\varepsilon}^{\prime}(0)=(0,0,1)=\mathcal{N}(\delta(0))$.

Isto completa a demonstração do lema.

O teorema 3.2.4, abaixo, classifica o ponto umbílico dado pelo lema 3.2 .3 .

Teorema 3.2.4. Seja $\delta\left(u_{0}\right)$ um ponto de tangência quadrática das superfícies $\mathbf{S}^{+} e \mathbf{S}^{-}$ tal que $\tau_{g}\left(u_{0}\right)=0, \tau_{g}^{\prime}\left(u_{0}\right) \neq 0$ e suponha que $k^{+}\left(u_{0}\right)<k_{n}\left(u_{0}\right)<k^{-}\left(u_{0}\right)$. Vimos (no lema 3.2.3) que a curva de pontos umbilicos $X\left(U_{\varepsilon}\right)$ é tangente ao vetor normal $\mathcal{N}\left(\delta\left(u_{0}\right)\right)$ e transversal à superfície $\mathbf{S}_{\varepsilon}$ em uma vizinhança de $\delta\left(u_{0}\right)$. Então, este ponto de interseção é um ponto umbílico Darbouxiano do tipo $D_{1}$ se $\tau_{g}^{\prime}\left(u_{0}\right)<0$ ou do tipo $D_{3}$ se $\tau_{g}^{\prime}\left(u_{0}\right)>0$.

Demonstração. Assim como na demonstração do lema 3.2 .3 defina a seguinte função:

$$
H(w)=\phi(w)+2 w \phi^{\prime}(w)+\frac{1}{2} w^{2} \phi^{\prime \prime}(w) .
$$

Os pontos umbílicos da superfície $\mathbf{S}_{\varepsilon}$ são dados pelas equações em (3.15), $L(u, w, \varepsilon)=M(u, w, \varepsilon)=N(u, w, \varepsilon)=0$.

Pelo lema 3.2.3 próximo do ponto umbílico $\left(0, w_{1}\right)$ segue que

$$
\begin{aligned}
L(u, w, \varepsilon) & =-\varepsilon^{8} \tau_{g}^{\prime}(0) u+O(2) ; \\
M(u, w, \varepsilon) & =\varepsilon^{6}\left(k^{+}-k_{n}\right)^{\prime}(0) u+\varepsilon^{6}\left(k^{-}-k^{+}\right)(0) H^{\prime}\left(w_{1}\right)\left(w-w_{1}\right)+O(2) ; \\
N(u, w, \varepsilon) & =\varepsilon^{4} \tau_{g}^{\prime}(0) u+O(2) .
\end{aligned}
$$

A equação diferencial das linhas de curvatura de $\mathbf{S}_{\varepsilon}$ na vizinhança do ponto umbílico $\left(0, w_{1}\right)$ é dada por:

$$
\begin{aligned}
{\left[-\varepsilon^{8} \tau_{g}^{\prime}(0) u+O(2)\right] d w^{2}+} & {\left[\varepsilon^{6}\left(k^{+}-k_{n}\right)^{\prime}(0) u+\varepsilon^{6} b_{0}\left(w-w_{1}\right)+O(2)\right] d u d w+} \\
& +\left[\varepsilon^{4} \tau_{g}^{\prime}(0) u+O(2)\right] d u^{2}=0
\end{aligned}
$$

onde $b_{0}=\left(k^{-}-k^{+}\right)(0) H^{\prime}\left(w_{1}\right)<0$.

Retornando às coordenadas originais $(u, v, \varepsilon)$, lembrando que $v=\varepsilon^{2} w, \varepsilon>0$ sufici- 
entemente pequeno, segue que $w=\varepsilon^{-2} v, d w=\varepsilon^{-2} d v$ e a equação 3.18 torna-se

$$
\left[-\varepsilon^{2} \tau_{g}^{\prime}(0) u+O(2)\right] d v^{2}+\left[\varepsilon^{2} k_{0} u+b_{0}\left(v-v_{1}\right)+O(2)\right] d u d v+\left[\varepsilon^{2} \tau_{g}^{\prime}(0) u+O(2)\right] d u^{2}=0
$$

sendo $k_{0}=\left(k^{+}-k_{n}\right)^{\prime}(0)$ e $w_{1}=\varepsilon^{-2} v_{1}$.

Pela classificação dos pontos umbílicos Darbouxianos, teorema 1.2 .3 do capítulo 1 , segue que a reta tangente às separatrizes umbílicas são definidas por $u=\lambda v$, onde

$$
\lambda\left[\varepsilon^{2} \tau_{g}^{\prime}(0) \lambda^{2}+\varepsilon^{2} k_{0} \lambda-\varepsilon^{2} \tau_{g}^{\prime}(0)+b_{0}\right]=0
$$

Portanto o discriminante desta equação é dado por

$$
\Delta=\varepsilon^{2}\left[\varepsilon^{2} k_{0}^{2}+4 \varepsilon\left(\tau_{g}^{\prime}(0)\right)^{2}-4 b_{0} \tau_{g}^{\prime}(0)\right]
$$

Como $b_{0}<0$, o sinal do discriminante $\Delta$ é determinado pelo sinal de $\tau_{g}^{\prime}(0)$. Assim, se $\tau_{g}^{\prime}(0)>0$, segue que $\Delta>0$ e o ponto umbílico é Darbouxiano do tipo $D_{3}$. Por outro lado, se $\tau_{g}^{\prime}(0)<0$, temos que $\Delta<0$ e o ponto umbílico é Darbouxiano do tipo $D_{1}$. Veja figura 3.3 .

Isto completa a demonstração.

\subsection{Pontos de Dobras e Cruzamentos Transversais}

\subsubsection{Ponto de Dobra}

Vimos na definição 2.1 .10 do capítulo 2 que um ponto sobre a curva bordo comum $\mathbf{B}$ é um ponto de B-dobra se é um ponto de tangência quadrática das linhas de curvatura da superfície $\mathbf{S}^{+}$(respectivamente $\mathbf{S}^{-}$) com $\mathbf{B}$ e um ponto de cruzamento transversal das linhas de curvatura da superfície $\mathbf{S}^{-}$(respectivamente $\mathbf{S}^{+}$) com $\mathbf{B}$.

Das proposições 3.1.2 e 3.1.4 sabemos que $\delta\left(u_{0}\right)$ será um ponto de cruzamento transversal das linhas de curvatura principal da superfície $\mathbf{S}^{+}$com $\mathbf{B}$ se $\tau_{g}\left(u_{0}\right) \neq 0$ e será um ponto de tangência quadrática das linhas de curvatura principal da superfície $\mathbf{S}^{-}$com $\mathbf{B}$ se $\tau_{g}\left(u_{0}\right)=0$. Portanto, não é possível termos um ponto de $\mathbf{B}$-dobra neste contexto.

\subsubsection{Cruzamentos Transversais}

Supondo $\tau_{g}\left(u_{0}\right) \neq 0$ teremos que as linhas de curvatura principal das superfícies $\mathbf{S}^{+}$e $\mathbf{S}^{-}$são transversais ao bordo $\mathbf{B}$ em uma vizinhança do ponto $\delta\left(u_{0}\right)$.

Definamos $\theta^{+}$(respectivamente $\theta^{-}$) como sendo o ângulo entre o eixo- $u$ positivo e a linha principal mínima da superfície $\mathbf{S}^{+}$(respectivamente $\mathbf{S}^{-}$) transversal à curva $\mathbf{B}$ em $\delta\left(u_{0}\right)$. Considere ainda o ângulo $\omega \in(0, \pi)$, entre a linha principal mínima da superfície 
$\mathbf{S}^{+}$e a linha principal mínima da superfície $\mathbf{S}^{-}$(veja figura 3.5). Como já discutimos no capítulo2, proposição 2.1.8, uma condição necessária para a existência de pontos umbílicos sobre a regularização da superfície $\mathbf{S}=\mathbf{S}^{+} \cup \mathbf{B} \cup \mathbf{S}^{-}$é que tenhamos sobre $\mathbf{S}$ um ponto de transição (definição 2.1.5 Neste ponto, por definição, as linhas de curvatura principais mínimas (e máximas) de $\mathbf{S}^{+}$e $\mathbf{S}^{-}$são ortogonais

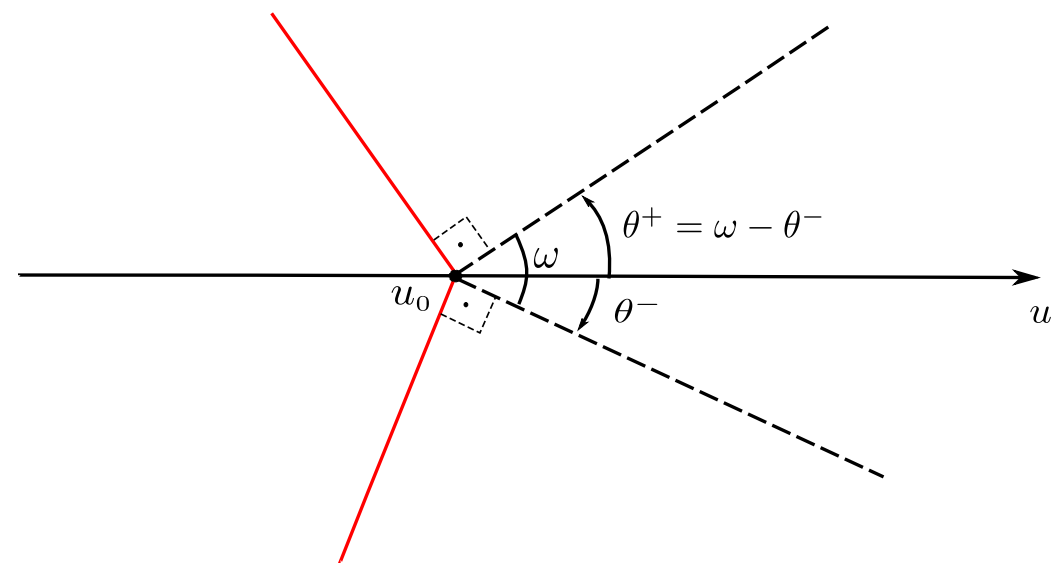

Figura 3.5: Exemplos de ângulos formados entre as linhas principais minimas (linhas tracejadas) e as linhas principais máximas (linhas contínuas) de $\mathbf{S}^{+}$e de $\mathbf{S}^{-}$com o eixo-u positivo.

Vamos determinar as curvaturas principais das superfícies $\mathbf{S}^{+}$e $\mathbf{S}^{-}$em $(u, v)=\left(u_{0}, 0\right)$ e, posteriormente, usando o sistema (1.3) do capítulo 1, encontraremos as condições para que $\delta\left(u_{0}\right)$ seja um ponto de transição.

No que segue, $k_{(1,+)} \leq k_{(2,+)}$ são as curvaturas principais da superfície $\mathbf{S}^{+}$em $\left(u_{0}, 0\right)$ e $k_{(1,-)} \leq k_{(2,-)}$ são as curvaturas principais da superfície $\mathbf{S}^{-}$em $\left(u_{0}, 0\right)$. Das expressões de $E, F, G, e, f$ e $g$ encontradas em (3.4) e (3.8) temos:

$$
\begin{aligned}
& k_{(1,+)}\left(u_{0}, 0\right)=\frac{1}{2}\left(k_{n}\left(u_{0}\right)+k^{+}\left(u_{0}\right)-\sqrt{\left[k^{+}\left(u_{0}\right)-k_{n}\left(u_{0}\right)\right]^{2}+4\left(\tau_{g}\left(u_{0}\right)\right)^{2}}\right), \\
& k_{(2,+)}\left(u_{0}, 0\right)=\frac{1}{2}\left(k_{n}\left(u_{0}\right)+k^{+}\left(u_{0}\right)+\sqrt{\left[k^{+}\left(u_{0}\right)-k_{n}\left(u_{0}\right)\right]^{2}+4\left(\tau_{g}\left(u_{0}\right)\right)^{2}}\right), \\
& k_{(1,-)}\left(u_{0}, 0\right)=\frac{1}{2}\left(k_{n}\left(u_{0}\right)+k^{-}\left(u_{0}\right)-\sqrt{\left[k^{-}\left(u_{0}\right)-k_{n}\left(u_{0}\right)\right]^{2}+4\left(\tau_{g}\left(u_{0}\right)\right)^{2}}\right), \\
& k_{(2,-)}\left(u_{0}, 0\right)=\frac{1}{2}\left(k_{n}\left(u_{0}\right)+k^{-}\left(u_{0}\right)+\sqrt{\left[k^{-}\left(u_{0}\right)-k_{n}\left(u_{0}\right)\right]^{2}+4\left(\tau_{g}\left(u_{0}\right)\right)^{2}}\right) .
\end{aligned}
$$

${ }^{3}$ Lembremos que, em termos dos coeficientes das formas fundamentais, as curvaturas Gaussiana $(\mathcal{K})$ e Média $(\mathcal{H})$ são dadas respectivamente por:

$$
\mathcal{K}=k_{1} \cdot k_{2}=\frac{e g-f^{2}}{E G-F^{2}}, \quad \mathcal{H}=\frac{k_{1}+k_{2}}{2}=\frac{e G-2 f F+g E}{2\left(E G-F^{2}\right)} .
$$

onde $k_{1} \leq k_{2}$ são as curvaturas principais. Portanto, $k_{1}$ e $k_{2}$ podem ser expressas por:

$$
k_{1}=\mathcal{H}-\sqrt{\mathcal{H}^{2}-\mathcal{K}}, \quad k_{2}=\mathcal{H}+\sqrt{\mathcal{H}^{2}-\mathcal{K}} .
$$


Abaixo, omitiremos o ponto $u_{0}$ das funções $k^{+}\left(u_{0}\right), k^{-}\left(u_{0}\right), k_{n}\left(u_{0}\right)$ e $\tau_{g}\left(u_{0}\right)$ a fim de simplificar a notação.

Do sistema (1.3) do capítulo 1 temos que as direções principais, relativas à superfície $\mathbf{S}^{+}$, são soluções do sistema:

$$
\left\{\begin{array}{l}
\left(e-k_{(i,+)} E\right) d u+\left(f-k_{(i,+)} F\right) d v=0 \\
\left(f-k_{(i,+)} F\right) d u+\left(g-k_{(i,+)} G\right) d v=0
\end{array}\right.
$$

onde $k_{(i,+)}(i=1,2)$ são as curvaturas principais determinadas em $(3.19)$. Agora, substituindo em (3.20) as expressões de $E\left(u_{0}, 0\right), F\left(u_{0}, 0\right), G\left(u_{0}, 0\right), e\left(u_{0}, 0\right), f\left(u_{0}, 0\right)$ e $g\left(u_{0}, 0\right)$ dadas por 3.4) e 3.8 encontramos dois sistemas:

Sistema que determina a direção principal mínima em $\mathbf{S}^{+}$:

$$
\left\{\begin{array}{l}
\frac{1}{2}\left(k_{n}-k^{+}+\sqrt{\left(k^{+}-k_{n}\right)^{2}+4\left(\tau_{g}\right)^{2}}\right) d u+\tau_{g} d v=0 \\
\tau_{g} d u+\frac{1}{2}\left(k^{+}-k_{n}+\sqrt{\left(k^{+}-k_{n}\right)^{2}+4\left(\tau_{g}\right)^{2}}\right) d v=0
\end{array}\right.
$$

Sistema que determina a direção principal máxima em $\mathbf{S}^{+}$:

$$
\left\{\begin{array}{l}
\frac{1}{2}\left(k_{n}-k^{+}-\sqrt{\left(k^{+}-k_{n}\right)^{2}+4\left(\tau_{g}\right)^{2}}\right) d u+\tau_{g} d v=0 \\
\tau_{g} d u+\frac{1}{2}\left(k^{+}-k_{n}-\sqrt{\left(k^{+}-k_{n}\right)^{2}+4\left(\tau_{g}\right)^{2}}\right) d v=0
\end{array} .\right.
$$

Analogamente ao que foi feito para a superfície $\mathbf{S}^{+}$temos que as direções principais, relativas à superfície $\mathbf{S}^{-}$, são soluções do sistema:

$$
\left\{\begin{array}{l}
\left(e-k_{(i,-)} E\right) d u+\left(f-k_{(i,-)} F\right) d v=0 \\
\left(f-k_{(i,-)} F\right) d u+\left(g-k_{(i,-)} G\right) d v=0
\end{array},\right.
$$

onde $k_{(i,-)}(i=1,2)$ são as curvaturas principais determinadas em $(3.19)$. Agora, substituindo em (3.23) as expressões de $E\left(u_{0}, 0\right), F\left(u_{0}, 0\right), G\left(u_{0}, 0\right), e\left(u_{0}, 0\right), f\left(u_{0}, 0\right)$ e $g\left(u_{0}, 0\right)$ dadas por 3.4 e (3.8) temos dois sistemas:

Sistema que determina a direção principal mínima em $\mathbf{S}^{-}$:

$$
\left\{\begin{array}{l}
\frac{1}{2}\left(k_{n}-k^{-}+\sqrt{\left(k^{-}-k_{n}\right)^{2}+4\left(\tau_{g}\right)^{2}}\right) d u+\tau_{g} d v=0 \\
\tau_{g} d u+\frac{1}{2}\left(k^{-}-k_{n}+\sqrt{\left(k^{-}-k_{n}\right)^{2}+4\left(\tau_{g}\right)^{2}}\right) d v=0
\end{array} .\right.
$$


Sistema que determina a direção principal máxima em $\mathbf{S}^{-}$:

$$
\left\{\begin{array}{l}
\frac{1}{2}\left(k_{n}-k^{-}-\sqrt{\left(k^{-}-k_{n}\right)^{2}+4\left(\tau_{g}\right)^{2}}\right) d u+\tau_{g} d v=0 \\
\tau_{g} d u+\frac{1}{2}\left(k^{-}-k_{n}-\sqrt{\left(k^{-}-k_{n}\right)^{2}+4\left(\tau_{g}\right)^{2}}\right) d v=0
\end{array} .\right.
$$

Como $\theta^{+}$(respectivamente $\theta^{-}$) é o ângulo entre o eixo- $u$ positivo e a linha principal mínima da superfície $\mathbf{S}^{+}$(respectivamente $\mathbf{S}^{-}$), temos que $\operatorname{tg}\left(\theta^{+}\right)=d v / d u$ (respectivamente $\operatorname{tg}\left(\theta^{-}\right)=d v / d u$ ) é dada por qualquer uma das equações do sistema (3.21) (respectivamente (3.24) ) acima.

Observe que $\omega=\pi$ se, e somente se, $\tan \left(\theta^{+}\right) \cdot \tan \left(\theta^{-}\right)=-1$.

Da primeira equação do sistema (3.21) e do sistema 3.24) (também poderíamos ter escolhido a segunda equação destes sistemas) obtemos que

$$
\begin{aligned}
& \tan \left(\theta^{+}\right)=\frac{d v}{d u}=-\frac{k_{n}-k^{+}+\sqrt{\left(k^{+}-k_{n}\right)^{2}+4\left(\tau_{g}\right)^{2}}}{2 \tau_{g}}, \\
& \tan \left(\theta^{-}\right)=\frac{d v}{d u}=-\frac{k_{n}-k^{-}+\sqrt{\left(k^{-}-k_{n}\right)^{2}+4\left(\tau_{g}\right)^{2}}}{2 \tau_{g}} .
\end{aligned}
$$

Portanto, $\tan \left(\theta^{+}\right) \cdot \tan \left(\theta^{-}\right)=-1$ se, e somente se,

$$
\frac{k_{n}-k^{+}+\sqrt{\left(k_{n}-k^{+}\right)^{2}+4\left(\tau_{g}\right)^{2}}}{\tau_{g}} \cdot \frac{k_{n}-k^{-}+\sqrt{\left(k_{n}-k^{-}\right)^{2}+4\left(\tau_{g}\right)^{2}}}{\tau_{g}}=-4 .
$$

Supondo, sem perda de generalidade, que $\tau_{g}>0$, a equação 3.26 se escreve como:

$$
\left[\frac{k_{n}-k^{+}}{\tau_{g}}+\sqrt{\left(\frac{k_{n}-k^{+}}{\tau_{g}}\right)^{2}+4}\right]\left[\frac{k_{n}-k^{-}}{\tau_{g}}+\sqrt{\left(\frac{k_{n}-k^{-}}{\tau_{g}}\right)^{2}+4}\right]=-4 .
$$

Observe que não exitem valores para $k^{+}, k^{-}, k_{n}$ e $\tau_{g}$ que resolvem a equação (3.27). Portanto, as linhas principais mínimas das superfícies $\mathbf{S}^{+}$e $\mathbf{S}^{-}$nunca serão ortogonais. Baseados nestas conclusões podemos enunciar o seguinte teorema.

Teorema 3.3.1. Considere a superficie $\mathbf{S}=\mathbf{S}^{+} \cup \mathbf{B} \cup \mathbf{S}^{-}$e sua $\varepsilon$-regularização $\mathbf{S}_{\varepsilon}$. Suponha que $\delta\left(u_{0}\right)$ seja um ponto de cruzamento transversal tal que $\tau_{g}\left(u_{0}\right) \neq 0$ e seja $\phi$ uma função de transição. Então sobre $\mathbf{S}_{\varepsilon}$ não teremos pontos umbílicos em uma vizinhança de $\left(u_{0}, 0\right)$, para todo $\varepsilon>0$.

Demonstração. Das expressões para $L(u, v), M(u, v)$ e $N(u, v)$ em 3.15) concluímos que $L(u, v)$ e $N(u, v)$ nunca se anulam para $(u, v)$ próximo de $\left(u_{0}, v\right)$ considerando $\tau_{g}\left(u_{0}\right) \neq 0$ e $\varepsilon>0$ pequeno. Portanto, sobre $\mathbf{S}_{\varepsilon}$ não teremos pontos umbílicos. 
Isto completa a demonstração.

A figura 3.6 representa um ponto de cruzamento transversal e sua $\varepsilon$-regularização. A região de regularização foi feita em grande escala para facilitar a visualização das linhas de curvatura principal da superfície regularizada, $\mathbf{S}_{\varepsilon}$, na vizinhança da origem.

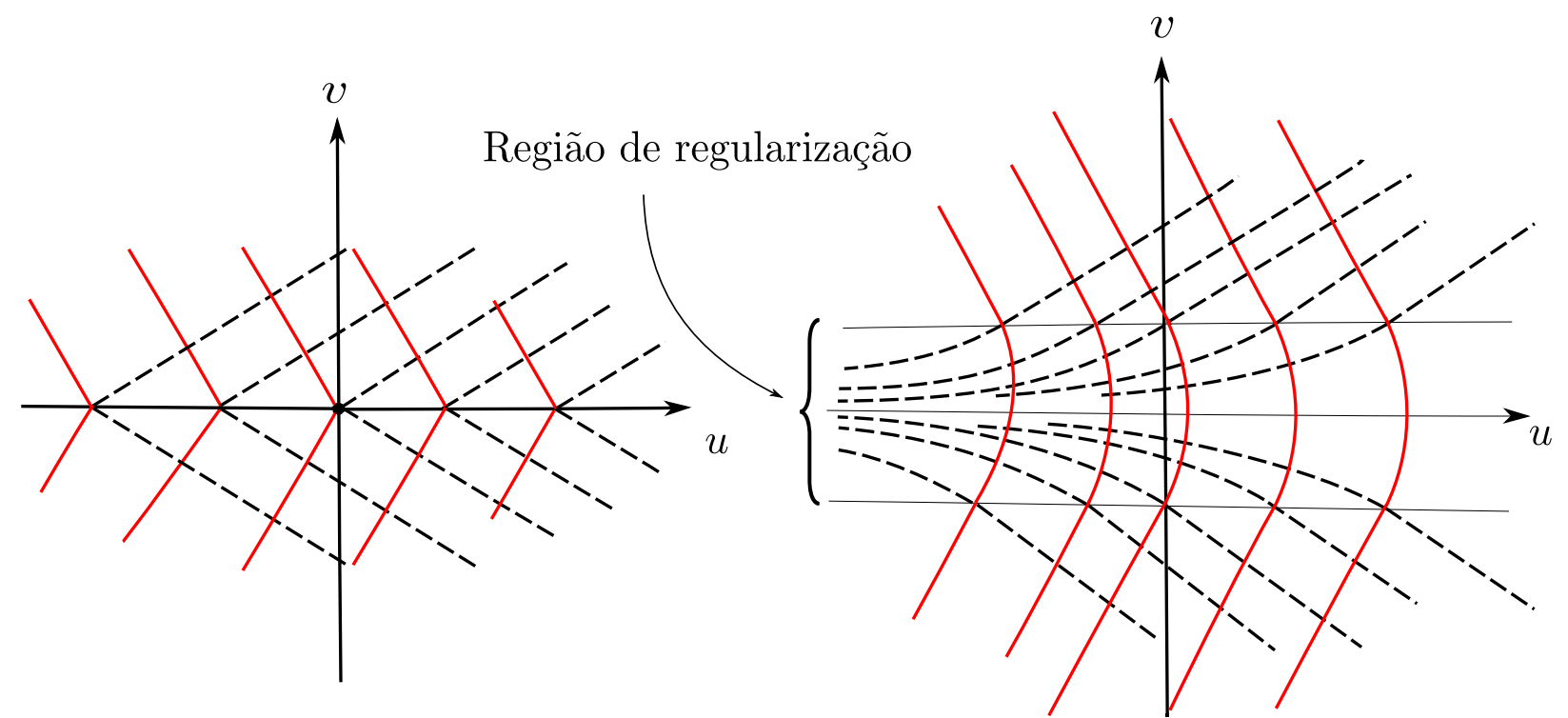

Figura 3.6: $\grave{A}$ esquerda, as linhas de curvatura principal de $\mathbf{S}^{+} e \mathbf{S}^{-}$são transversais ao bordo comum $\mathbf{B}$ e, à direita, a $\varepsilon$-regularização de $\mathbf{S}$. 


\section{Capítulo 4}

\section{Configuração Principal sobre Superfícies com Bordo em $\mathbb{R}^{3}$ : Contato Cúbico e Pontos Umbílicos}

Neste capítulo serão estudadas as linhas de curvatura principal de uma superfície $\mathbf{S}$ em $\mathbb{R}^{3}$ com bordo $\mathbf{B}$ e da superfície suave, obtida de $\mathbf{S}$, através do engrossamento e regularização definidos por Garcia e Sotomayor em [5].

Em [5], os autores consideraram o caso genérico, no qual a configuração principal da superfície com bordo é regular, ou seja, sem pontos umbílicos e sendo o contato de uma linha de curvatura principal com o bordo do tipo quadrático.

Analisaremos um caso complementar, chamado aqui de crítico, no qual o contato de uma linha de curvatura principal com o bordo é cúbico, dito tangencialmente crítico.

\subsection{Introdução}

Nesta seção, baseada em [5, 6], apresentaremos a operação de engrossamento de superfícies em $\mathbb{R}^{3}$ com bordo, a qual será aplicada para o estudo do caso tangencialmente crítico proposto acima.

Seja $\mathbf{S}$ uma superfície imersa em $\mathbb{R}^{3}$ com bordo $\mathbf{B}$, sendo $\mathbf{S}$ de classe $C^{\infty}$, compacta, conexa e orientada. Sem perda de generalidade, podemos assumir que $\mathbf{S}$ está contida em $\widehat{\mathbf{S}}$, sendo $\widehat{\mathbf{S}}$ uma superfície sem bordo definida implicitamente pela função suave $f: \mathbb{R}^{3} \rightarrow \mathbb{R}$ tal que

$$
\widehat{\mathbf{S}}=\left\{\mathfrak{p} \in \mathbb{R}^{3}: f(\mathfrak{p})=0\right\}
$$


e sendo $\mathbf{S}$ e $\mathbf{B}$ dados, em termos de uma função suave $b: \mathbb{R}^{3} \rightarrow \mathbb{R}$, respectivamente por:

$$
\begin{aligned}
\mathbf{S} & =\{\mathfrak{p} \in \widehat{\mathbf{S}}: b(\mathfrak{p}) \geq 0\}, \\
\mathbf{B} & =\{\mathfrak{p} \in \widehat{\mathbf{S}}: b(\mathfrak{p})=0\},
\end{aligned}
$$

com $\nabla f \neq 0$ sobre $\widehat{\mathbf{S}}$ e $\widehat{T}=\nabla f \wedge \nabla b \neq 0$ sobre $\mathbf{B}$.

A função $f$ é definida de tal forma que a aplicação normal unitária positiva da superfície $\mathbf{S}$ é dada por:

$$
\mathcal{N}=\frac{\nabla f}{|\nabla f|}
$$

A orientação positiva sobre $\mathbf{B}$ é definida pelo campo de vetores tangente unitário $T=\frac{\widehat{T}}{|\widehat{T}|}$. Diremos, neste caso, que B está munido da orientação externa.

A configuração principal do par $(\mathbf{S}, \mathbf{B})$, consistindo da superfície orientada $\mathbf{S}$ e do bordo orientado $\mathbf{B}$, é definida pela quádrupla

$$
\mathcal{P}_{(\mathbf{S}, \mathbf{B})}=\left(\mathcal{U}_{\mathbf{S}}, \mathcal{C}_{\mathbf{S}}, \mathcal{F}_{1, \mathbf{S}}, \mathcal{F}_{2, \mathbf{S}}\right)
$$

onde $\mathcal{U}_{\mathbf{S}}$ é o conjunto dos pontos umbílicos de $\mathbf{S}, \mathcal{C}_{\mathbf{S}}$ é o conjunto das singularidades tangenciais sobre $\mathbf{B}$ ( $T$ é uma direção principal), $\mathcal{F}_{1, \mathbf{S}}$ e $\mathcal{F}_{2, \mathbf{S}}$ são, respectivamente, as folheações principais mínima e máxima sobre $\mathbf{S} \backslash \mathcal{U}_{\mathbf{S}} \cup \mathcal{C}_{\mathbf{S}}$.

\subsubsection{Envelopes de Superfícies Regulares}

Uma família a 1 parâmetro de superfícies regulares em $\mathbb{R}^{3}$ pode ser definida por $F(\mathfrak{p}, \lambda)=0$, onde $F: \mathbb{R}^{3} \times \mathbb{R} \longrightarrow \mathbb{R}$ é tal que, para cada $\lambda, \nabla F_{\lambda} \neq 0$, sendo $F_{\lambda}(\cdot)=F(\cdot, \lambda)$.

A variação desta família em relação ao parâmetro $\lambda$ pode ser definida como $\frac{\partial F}{\partial \lambda}(\mathfrak{p}, \lambda)$. O conjunto

$$
\mathcal{C}:=\left\{(\mathfrak{p}, \lambda) \in \mathbb{R}^{3} \times \mathbb{R} ; F(\mathfrak{p}, \lambda)=\frac{\partial F}{\partial \lambda}(\mathfrak{p}, \lambda)=0\right\}
$$

é chamado conjunto característico desta família.

A projeção $\pi_{1}(\mathcal{C})=\mathbb{E}$ é o envelope da família, onde $\pi_{1}: \mathbb{R}^{3} \times \mathbb{R} \longrightarrow \mathbb{R}, \pi_{1}(\mathfrak{p}, \lambda)=\mathfrak{p}$.

Exemplo 4.1.1. Considere uma família a 1 parâmetro de esferas de raios constantes $r>0$ com centro variando ao longo de uma curva $c(s)$. Esta família pode ser representada por

$$
F(\mathfrak{p}, s)=|\mathfrak{p}-c(s)|^{2}-r^{2}=0 .
$$

Portanto, segue que $\frac{\partial F}{\partial s}(\mathfrak{p}, s)=-2\left\langle\mathfrak{p}-c(s), c^{\prime}(s)\right\rangle$. 
O envelope $\mathbb{E}$ desta família é chamado de superfície canal.

Definição 4.1.2. Seja $\mathbf{S}$ uma superfície em $\mathbb{R}^{3}$ com bordo B. O E-engrossamento de $\mathbf{S}$ é a vizinhança tubular $\mathbf{T} \mathbf{S}_{\varepsilon}$ de $\mathbf{S}$, onde

$$
\mathbf{T S}_{\varepsilon}=\left\{q \in \mathbb{R}^{3}:|q-p| \leq \varepsilon, p \in \mathbf{S}\right\}
$$

Para $\varepsilon>0$ suficientemente pequeno, $\mathbf{S}_{\varepsilon}=\partial\left(\mathbf{T S}_{\varepsilon}\right)$ é o envelope da família de esferas de raio $\varepsilon$ e centro variando sobre $\mathbf{S}$.

A superfície $\mathbf{S}_{\varepsilon}$ é compacta, de classe $C^{1}$ e orientada pelo normal exterior à $\mathbf{S}_{\varepsilon}$.

Observe que, para $\varepsilon>0$ pequeno o suficiente, $\mathbf{S}_{\varepsilon}$ é uma superfície obtida pela justaposição da superfície $\mathbf{S}_{\varepsilon,+}=\mathbf{S}+\varepsilon \mathcal{N}$, do semitubo $\mathbf{T}_{\varepsilon}$ e da superfície $\mathbf{S}_{\varepsilon,-}=\mathbf{S}-\varepsilon \mathcal{N}$, sendo $\mathbf{T}_{\varepsilon}$ um tubo de raio $\varepsilon>0$ com centro variando sobre o bordo $\mathbf{B}$ e definido parametricamente por:

$$
\mathbf{T}_{\varepsilon}=\mathbf{B}+\varepsilon \operatorname{sen} \theta(\mathcal{N} \wedge T)+\varepsilon \cos \theta \mathcal{N}, \quad 0 \leq \theta \leq \pi
$$

Vamos denotar por $\mathbf{B}_{\varepsilon,+}$ (respectivamente $\mathbf{B}_{\varepsilon,-}$ ) o bordo comum à superfície $\mathbf{S}_{\varepsilon,+}$ (respectivamente $\mathbf{S}_{\varepsilon,-}$ ) e ao semitubo $T_{\varepsilon}$. Destas considerações podemos concluir que a superfície $\mathbf{S}_{\varepsilon}$ é de classe $C^{\infty}$ sobre o complementar das curvas $\mathbf{B}_{\varepsilon, \pm}$. Veja na figura 4.1 uma ilustração local da superfície $\mathbf{S}_{\varepsilon}$.

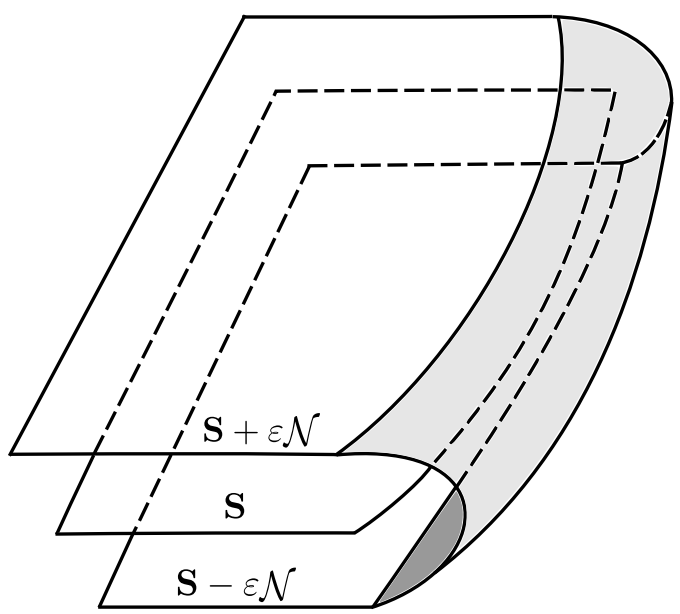

Figura 4.1: Um aberto do E-engrossamento da superfície $\mathbf{S}$.

\subsection{Configuração Principal de $\mathbf{S}_{\varepsilon}=\mathbf{S}_{\varepsilon,+} \cup \mathbf{T}_{\varepsilon} \cup \mathbf{S}_{\varepsilon,-}$}

Usando a mesma notação da seção 4.1. seja (S, B) o par consistindo da superfície orientada $\mathbf{S}$ e do bordo orientado externamente $\mathbf{B}$ e seja $\mathbf{S}_{\varepsilon}$ a superfície definida por $\mathbf{S}_{\varepsilon}=\mathbf{S}_{\varepsilon,+} \cup \mathbf{T}_{\varepsilon} \cup \mathbf{S}_{\varepsilon,-}, \quad \varepsilon>0$ suficientemente pequeno. 
A proposição 4.2.1, seguinte, apresenta uma parametrização para uma vizinhança de uma componente conexa da curva bordo $\mathbf{B}$.

Proposição 4.2.1. Considere $\delta:[0, l] \longrightarrow \mathbb{R}^{3}$ uma parametrização pelo comprimento de arco de uma componente conexa de $\mathbf{B}$ tal que $\{T, \mathcal{N} \wedge T, \mathcal{N}\}$ é um referencial móvel ao longo de $\delta$, sendo $T(u)$ o vetor tangente unitário à curva $u \longmapsto \delta(u)$. Então, a expressão $X(u, v)=\delta(u)+v(\mathcal{N} \wedge T)(u)+\left[\frac{1}{2} k_{n}^{\perp}(u) v^{2}+\frac{1}{6} A(u) v^{3}+C(u, v) v^{3}\right] \mathcal{N}(u),-\mu<v<\mu$

define uma carta local $(u, v)$ sobre a superfície $\widehat{\mathbf{S}}$, de classe $C^{\infty}$ em uma vizinhança de $\delta$, onde $C(u, 0)=0$ e $k_{n}^{\perp}$ é a curvatura normal de $\mathbf{S}$ na direção de $B=\mathcal{N} \wedge T$.

Demonstração. Análoga à da proposição 3.1.1 do capítulo 3.

O referencial de Darboux $\{T, B=\mathcal{N} \wedge T, \mathcal{N}\}$ ao longo de $\mathbf{B}$ satisfaz o seguinte sistema de equações diferenciais:

$$
\begin{gathered}
T^{\prime}(u)=k_{g}(u) B(u)+k_{n}(u) \mathcal{N}(u), \\
B^{\prime}(u)=-k_{g}(u) T(u)+\tau_{g}(u) \mathcal{N}(u), \\
\mathcal{N}^{\prime}(u)=-k_{n}(u) T(u)-\tau_{g}(u) B(u),
\end{gathered}
$$

onde $k_{n}(u)$ é a curvatura normal, $k_{g}(u)$ é a curvatura geodésica e $\tau_{g}(u)$ é a torção geodésica da curva bordo $\mathbf{B}$ contida em $\mathbf{S}$.

A proposição 4.2.2, abaixo, estabelece as condições para que as linhas de curvatura principal da superfície $\mathbf{S}$ tenham contato cúbico com o bordo $\mathbf{B}$ na vizinhança do ponto $\delta\left(u_{0}\right)$.

Proposição 4.2.2. Considere a superfície $\mathbf{S}$ parametrizada, em uma vizinhança do ponto $\left(u_{0}, 0\right)$, por $X(u, v)=\delta(u)+v B(u)+\left[\frac{1}{2} k_{n}^{\perp}(u) v^{2}+\frac{1}{6} A(u) v^{3}+C(u, v) v^{3}\right] \mathcal{N}(u)$, $-\mu<v<\mu$, sendo $\delta$ uma parametrização pelo comprimento de arco u de uma componente conexa da curva bordo $\mathbf{B}$.

Supondo que $\left(k_{n}^{\perp}-k_{n}\right)\left(u_{0}\right)>0$, uma linha de curvatura principal minima tem contato cúbico com $\delta$ em $\delta\left(u_{0}\right)$ se, e somente se, $\tau_{g}\left(u_{0}\right)=\tau_{g}^{\prime}\left(u_{0}\right)=0$ e $\tau_{g}^{\prime \prime}\left(u_{0}\right) \neq 0$.

Demonstração. Usando a parametrização da superfície $\mathbf{S}$ dada por 4.1) e o referencial de Darboux ao longo de B satisfazendo as equações (4.2) temos que os coeficientes da Primeira e Segunda Formas Fundamentais em $(u, 0)$ são:

$$
\begin{aligned}
E(u, 0) & =1, & F(u, 0) & =0, & G(u, 0) & =1, \\
e(u, 0) & =k_{n}(u), & f(u, 0) & =\tau_{g}(u), & g(u, 0) & =k_{n}^{\perp}(u) .
\end{aligned}
$$


As direções principais de $\mathbf{S}$ em $(u, 0)$ satisfazem a equação:

$$
-\tau_{g}(u) d v^{2}+\left(k_{n}^{\perp}-k_{n}\right)(u) d u d v+\tau_{g}(u) d u^{2}=0 .
$$

Portanto, o vetor $T(u)$ é uma direção principal de 4.3 se, e somente se, $\tau_{g}(u)=0$.

Próximo ao ponto $\left(u_{0}, 0\right)$, a equação diferencial das linhas de curvatura de $\mathbf{S}$ é dada por

$$
L(u, v) d v^{2}+M(u, v) d u d v+N(u, v) d u^{2}=0
$$

sendo

$$
\begin{aligned}
L(u, v) & =(F g-G f)(u, v)=-\tau_{g}(u)-\left(k_{n}^{\perp}(u)\right)^{\prime} v+\left[\frac{1}{2} k_{g}(u)\left(k_{n}^{\perp}(u)\right)^{\prime}-\right. \\
& \left.-\frac{1}{2} k_{n}(u) k_{n}^{\perp}(u) \tau_{g}(u)-\frac{1}{2} A^{\prime}(u)-3 C_{u}(u, v)-\frac{3}{2} \tau_{g}(u)\left(k_{n}^{\perp}(u)\right)^{\prime}\right] v^{2}+v^{3}(\cdots), \\
M(u, v) & =(E g-G e)(u, v)=k_{n}^{\perp}(u)-k_{n}(u)+\left[-\tau_{g}^{\prime}(u)+6 C(u, v)+A(u)+\right. \\
& \left.+2 k_{g}(u) k_{n}(u)-2 k_{g}(u) k_{n}^{\perp}(u)\right] v+\left\{-\frac{5}{2} k_{n}(u)\left(k_{n}^{\perp}(u)\right)^{2}+\right. \\
& +\left[k_{n}^{2}(u)+\frac{1}{2} \tau_{g}^{2}(u)+k_{g}^{2}(u)\right] k_{n}^{\perp}(u)-k_{g}^{2}(u) k_{n}(u)-\frac{1}{2}\left(k_{n}^{\perp}(u)\right)^{\prime \prime}+ \\
& +\left[\tau_{g}^{\prime}(u)-\frac{5}{2} A(u)-15 C(u, v)\right] k_{g}(u)+6 C_{v}(u, v)- \\
& \left.-\tau_{g}^{2}(u) k_{n}(u)-\tau_{g}(u) k_{g}^{\prime}(u)\right\} v^{2}+v^{3}(\cdots), \\
N(u, v) & =(E f-F e)(u, v)=\tau_{g}(u)+\left[-2 k_{g}(u) \tau_{g}(u)+\left(k_{n}^{\perp}(u)\right)^{\prime}\right] v+ \\
+ & {\left[3 C_{u}(u, v)-k_{n}^{\perp}(u) k_{n}(u) \tau_{g}(u)+\tau_{g}(u)\left(k_{n}^{\perp}(u)\right)^{2}-\right.} \\
& \left.-\frac{5}{2} k_{g}(u)\left(k_{n}^{\perp}(u)\right)^{\prime}+\tau_{g}^{3}(u)+\frac{1}{2} A^{\prime}(u)+k_{g}^{2}(u) \tau_{g}(u)\right] v^{2}+v^{3}(\cdots) .
\end{aligned}
$$

Usando o "Método dos Coeficientes a Determinar" temos que, na vizinhança do ponto $\left(u_{0}, 0\right)$, as linhas de curvatura principal são soluções de 4.4$)$ e descritas por

$$
v(u)=\frac{1}{6} \frac{\tau_{g}^{\prime \prime}\left(u_{0}\right)}{\left(k_{n}-k_{n}^{\perp}\right)\left(u_{0}\right)}\left(u-u_{0}\right)^{3}+\cdots, \quad-\mu<v<\mu .
$$

De fato, no "Método dos Coeficientes a Determinar" supomos que

$$
v=a_{0}+a_{1}\left(u-u_{0}\right)+\frac{a_{2}}{2}\left(u-u_{0}\right)^{2}+\frac{a_{3}}{6}\left(u-u_{0}\right)^{3}+\cdots
$$


é uma solução de (4.4). Para determinarmos os coeficientes $a_{i}, i=0,1,2, \cdots$ basta substituir

$$
v=a_{0}+a_{1}\left(u-u_{0}\right)+\frac{a_{2}}{2}\left(u-u_{0}\right)^{2}+\frac{a_{3}}{6}\left(u-u_{0}\right)^{3}+\cdots
$$

e

$$
d v=a_{1} d u+a_{2}\left(u-u_{0}\right) d u+\frac{1}{2} a_{3}\left(u-u_{0}\right)^{2} d u+\cdots
$$

na equação (4.4). Conhecida a expressão do coeficiente $a_{i-1}$, podemos determinar os próximos coeficientes $a_{i}, i=1,2, \cdots$.

Observe que da equação 4.3 já concluímos que se $\tau_{g}\left(u_{0}\right)=0$ então $a_{0}=a_{1}=0$. Após alguns cálculos, obtemos

$$
\begin{aligned}
a_{2} & =\frac{\tau_{g}^{\prime}\left(u_{0}\right)}{\left(k_{n}-k_{n}^{\perp}\right)\left(u_{0}\right)}, \\
a_{3} & =\frac{\tau_{g}^{\prime}\left(u_{0}\right)\left[3\left(k_{n}^{\perp}\right)^{\prime}-2 k_{n}^{\prime}\right]\left(u_{0}\right)+\tau_{g}^{\prime \prime}\left(u_{0}\right)\left[k_{n}-k_{n}^{\perp}\right]\left(u_{0}\right)}{\left(k_{n}-k_{n}^{\perp}\right)^{2}\left(u_{0}\right)},
\end{aligned}
$$

e assim por diante.

Portanto, em $\left(u_{0}, 0\right)$ uma linha principal mínima de $\mathbf{S}$ tem contato cúbico com $\mathbf{B}$ em $\delta\left(u_{0}\right)$ se, e somente se, $\tau_{g}\left(u_{0}\right)=\tau_{g}^{\prime}\left(u_{0}\right)=0$ e $\tau_{g}^{\prime \prime}\left(u_{0}\right) \neq 0$.

Isto completa a demonstração.

Observação 4.2.3. Na proposição 4.2.2 mudamos a orientação de $\mathbf{S}$ se considerarmos $\left(k_{n}^{\perp}-k_{n}\right)\left(u_{0}\right)<0$. Logo, supondo que $\tau_{g}\left(u_{0}\right)=\tau_{g}^{\prime}\left(u_{0}\right)=0$, uma linha de curvatura principal máxima terá contato cúbico com $\delta$ em $\delta\left(u_{0}\right)$ se, e somente se, $\tau_{g}\left(u_{0}\right)=\tau_{g}^{\prime}\left(u_{0}\right)=$ 0 e $\tau_{g}^{\prime \prime}\left(u_{0}\right) \neq 0$.

Veremos na proposição 4.2.4, a seguir, que as superfícies $\mathbf{S}$ e $\mathbf{S}_{\varepsilon,+}$ possuem as mesmas configurações principais.

Proposição 4.2.4 ([5], Proposição 3). Considere a superfície $\mathbf{S}$ parametrizada, em uma vizinhança de uma componente conexa do bordo $\mathbf{B}$, por

$$
X(u, v)=\delta(u)+v B(u)+\left[\frac{1}{2} k_{n}^{\perp}(u) v^{2}+\frac{1}{6} A(u) v^{3}+C(u, v) v^{3}\right] \mathcal{N}(u),-\mu<v<\mu .
$$

Sejam $\varepsilon>0$ suficientemente pequeno e $\mathbf{S}_{\varepsilon,+}$ superfícies paralelas definidas por

$$
X_{\varepsilon,+}(u, v)=X(u, v)+\varepsilon \mathcal{N}(u, v),
$$

onde $\mathcal{N}$ é a aplicação normal unitária que orienta positivamente a superfície $\mathbf{S}$.

Então, as configurações principais de $\mathbf{S} e \mathbf{S}_{\varepsilon,+}$ são equivalentes. Isto é, as linhas de 
curvatura principal, os pontos umbílicos e as tangências são preservadas por deslocamentos paralelos.

Demonstração. Sejam $(u, v)$ uma carta positiva arbitrária,

$$
(F g-G f)(u, v) d v^{2}+(E g-G e)(u, v) d u d v+(E f-F e)(u, v) d u^{2}=0
$$

a equação diferencial das linhas de curvatura de $\mathbf{S}$ e

$$
\left(F_{\varepsilon} g_{\varepsilon}-G_{\varepsilon} f_{\varepsilon}\right)(u, v) d v^{2}+\left(E_{\varepsilon} g_{\varepsilon}-G_{\varepsilon} e_{\varepsilon}\right)(u, v) d u d v+\left(E_{\varepsilon} f_{\varepsilon}-F_{\varepsilon} e_{\varepsilon}\right)(u, v) d u^{2}=0
$$

a equação diferencial das linhas de curvatura de $\mathbf{S}_{\varepsilon}$.

Cálculos diretos (veja [13], p.185) mostram que os coeficientes da Primeira e Segunda Formas Fundamentais de $\mathbf{S}$ e $\mathbf{S}_{\varepsilon}$ são expressos por:

$$
\begin{aligned}
E_{\varepsilon} & =(1-\varepsilon \mathcal{K}) E+\left(\varepsilon^{2} \mathcal{H}-2 \varepsilon\right) e, \\
F_{\varepsilon} & =(1-\varepsilon \mathcal{K}) F+\left(\varepsilon^{2} \mathcal{H}-2 \varepsilon\right) f, \\
G_{\varepsilon} & =(1-\varepsilon \mathcal{K}) G+\left(\varepsilon^{2} \mathcal{H}-2 \varepsilon\right) g, \\
e_{\varepsilon} & =(1-\varepsilon \mathcal{H}) e+\varepsilon \mathcal{K} E, \\
f_{\varepsilon} & =(1-\varepsilon \mathcal{H}) f+\varepsilon \mathcal{K} F, \\
g_{\varepsilon} & =(1-\varepsilon \mathcal{H}) g+\varepsilon \mathcal{K} G .
\end{aligned}
$$

Aqui $\mathcal{K}=k_{1} k_{2}$ e $\mathcal{H}=\frac{k_{1}+k_{2}}{2}$ são, respectivamente, as curvaturas Gaussiana e Média da superfície $\mathbf{S}$.

Portanto,

$$
\begin{aligned}
& \left(F_{\varepsilon} g_{\varepsilon}-G_{\varepsilon} f_{\varepsilon}\right)(u, v)=\left(1+\varepsilon^{2} \mathcal{K}-\varepsilon \mathcal{H}\right)(F g-G f), \\
& \left(E_{\varepsilon} g_{\varepsilon}-G_{\varepsilon} e_{\varepsilon}\right)(u, v)=\left(1+\varepsilon^{2} \mathcal{K}-\varepsilon \mathcal{H}\right)(E g-G e), \\
& \left(E_{\varepsilon} f_{\varepsilon}-F_{\varepsilon} e_{\varepsilon}\right)(u, v)=\left(1+\varepsilon^{2} \mathcal{K}-\varepsilon \mathcal{H}\right)(E f-F e) .
\end{aligned}
$$

Concluímos assim, que as equações diferenciais das linhas de curvatura das superfícies $\mathbf{S}$ e $\mathbf{S}_{\varepsilon}$ na carta $(u, v)$ são as mesmas, para todo $\varepsilon>0$ pequeno.

Isto completa a demonstração.

A proposição 4.2.5, abaixo, estabelece as hipóteses para que as linhas de curvatura principal do tubo $\mathbf{T}_{\varepsilon}$, de raio $\varepsilon>0$ e centro variando sobre a curva $\mathbf{B}$, tenham contato cúbico com as curvas definidas por $\theta=0$ e $\theta=\pi$. 
Proposição 4.2.5. Seja $\delta:[0, l] \longrightarrow \mathbb{R}^{3}$ uma parametrização pelo comprimento de arco u de uma componente conexa da curva bordo $\mathbf{B}$ tal que $\{T, B=\mathcal{N} \wedge T, \mathcal{N}\}$ é um referencial móvel ao longo de $\delta$. Então, a expressão

$$
\alpha(u, \theta)=\delta(u)+\varepsilon \operatorname{sen} \theta B(u)+\varepsilon \cos \theta \mathcal{N}(u)
$$

é uma parametrização regular do tubo $\mathbf{T}_{\varepsilon}$ de raio $\varepsilon>0$ e centro sobre a curva $\delta(u)$. A equação diferencial das linhas de curvatura principal de $\mathbf{T}_{\varepsilon}$ é dada por:

$$
d u\left[d \theta-\tau_{g}(u) d u\right]=0 .
$$

Em todos os pontos onde $\tau_{g}\left(u_{0}\right)=\tau_{g}^{\prime}\left(u_{0}\right)=0$ e $\tau_{g}^{\prime \prime}\left(u_{0}\right) \neq 0$, o contato entre as linhas de curvatura principal máxima e as curvas definidas por $\theta=0$ e $\theta=\pi$ é do tipo cúbico.

Demonstração. Para $\varepsilon>0$ suficientemente pequeno, o tubo $\mathbf{T}_{\varepsilon}$ é, claramente, uma superfície regular.

Usando a parametrização (4.5) e o referencial de Darboux (4.2), obtemos:

$$
\begin{aligned}
& \frac{\partial \alpha}{\partial u}(u, v)=\left[1-\varepsilon k_{n}(u) \cos \theta-\varepsilon k_{g}(u) \operatorname{sen} \theta\right] T(u)-\varepsilon \tau_{g}(u) \cos \theta B(u)+\varepsilon \tau_{g}(u) \operatorname{sen} \theta \mathcal{N}(u) \\
& \frac{\partial \alpha}{\partial \theta}(u, v)=\varepsilon \cos \theta B(u)-\varepsilon \operatorname{sen} \theta \mathcal{N}(u) \\
& \frac{\partial \alpha}{\partial u} \wedge \frac{\partial \alpha}{\partial \theta}=\varepsilon\left(1-\varepsilon k_{n}(u) \cos \theta-\varepsilon k_{g}(u) \operatorname{sen} \theta\right)[\operatorname{sen} \theta B(u)+\cos \theta \mathcal{N}(u)]
\end{aligned}
$$

Os coeficientes da Primeira Forma Fundamental de $\mathbf{T}_{\varepsilon}$, expressos na carta $(u, \theta)$, são:

$$
\begin{aligned}
& E(u, \theta)=\left[1-\varepsilon k_{n}(u) \cos \theta-\varepsilon k_{g}(u) \operatorname{sen} \theta\right]^{2}+\left(\varepsilon k_{g}(u)\right)^{2}+\left(\varepsilon \tau_{g}(u)\right)^{2}, \\
& F(u, \theta)=-\varepsilon^{2} \tau_{g}(u), \\
& G(u, \theta)=\varepsilon^{2} .
\end{aligned}
$$

O tubo $\mathbf{T}_{\varepsilon}$ é o envelope de uma família a 1 parâmetro de esferas de raio $\varepsilon$ e centro variando ao longo de $\delta$, veja exemplo 4.1.1. Os círculos parametrizados por $u=c t e$ são as linhas de curvatura principal mínima de $\mathbf{T}_{\varepsilon}$ (veja [8, 13]). Considerando que a orientação positiva do tubo é definida pelo seu normal exterior, temos que os círculos parametrizados por $u=$ cte têm curvatura principal mínima $k_{1}=-\frac{1}{\varepsilon}$.

Portanto, as linhas de curvatura principal máxima (família de curvas ortogonais aos círculos $u=$ cte) são soluções da seguinte equação diferencial

$$
\frac{d \theta}{d u}=-\frac{F}{G}=\tau_{g}(u)
$$


Logo, a equação diferencial das linhas de curvatura de $\mathbf{T}_{\varepsilon}$ é dada por $d u\left[d \theta-\tau_{g}(u) d u\right]=0$ e, nos pontos $u_{0}$ onde $\tau_{g}\left(u_{0}\right)=\tau_{g}^{\prime}\left(u_{0}\right)=0$ e $\tau_{g}^{\prime \prime}\left(u_{0}\right) \neq 0$, a solução da equação (4.6) tem a seguinte expansão de Taylor em torno do ponto $u_{0}$

$$
\theta(u)=\theta_{0}+\frac{1}{6} \tau_{g}^{\prime \prime}\left(u_{0}\right)\left(u-u_{0}\right)^{3}+\cdots
$$

Isto completa a demonstração.

Definição 4.2.6. Sejam $\mathbf{S}_{\varepsilon,+}$ e $\mathbf{S}_{\varepsilon,-}$ superfícies paralelas definidas, respectivamente, por $X_{\varepsilon,+}=X+\varepsilon \mathcal{N}$ e $X_{\varepsilon,-}=X-\varepsilon \mathcal{N}=X+\varepsilon(-\mathcal{N}), \varepsilon>0$. Considere $\mathbf{S}_{\varepsilon}=\mathbf{S}_{\varepsilon,+} \cup \mathbf{T}_{\varepsilon} \cup \mathbf{S}_{\varepsilon,-}$ a superfície sem bordo obtida justapondo-se a superfície $\mathbf{S}_{\varepsilon,+}, \quad$ o semitubo $\mathbf{T}_{\varepsilon}$ de raio $\varepsilon>0$ e centro variando ao longo do bordo $\mathbf{B}$ de $\mathbf{S}$ e a superfície $\mathbf{S}_{\varepsilon,-}$. Então, para $\varepsilon>0$ suficientemente pequeno, a superfície $\mathbf{S}_{\varepsilon}$ é suave no complementar das curvas $\mathbf{B}_{\varepsilon}=\mathbf{B} \pm \varepsilon \mathcal{N}$ e regular de classe $C^{1}$ ao longo destas curvas.

Para cada $\varepsilon>0$ suficientemente pequeno, as linhas de curvatura das folheações principais de $\mathbf{S}_{\varepsilon}$ são definidas como segue:

(I) As linhas principais mínimas de $\mathbf{S}_{\varepsilon}$, fora das tangências, são as linhas principais minimas da superficie $\mathbf{S}_{\varepsilon,+}$ justapostas com as linhas principais minimas do tubo $T_{\varepsilon}$ (semicírculos) e as linhas principais mínimas da superfície $\mathbf{S}_{\varepsilon,-}$. Observe que as linhas principais mínimas de $\mathbf{S}_{\varepsilon,-}$ são translações paralelas das linhas principais máximas de $\mathbf{S}$.

(II) As linhas principais máximas de $\mathbf{S}_{\varepsilon}$, fora das tangências, são as linhas principais máximas da superfície $\mathbf{S}_{\varepsilon,+}$ justapostas com as linhas principais máximas do tubo $T_{\varepsilon}$ (curvas definidas pela equação diferencial $d \theta-\tau_{g}(u) d u=0$, veja proposição 4.2.5) $e$ as linhas principais máximas da superfície $\mathbf{S}_{\varepsilon,-}$. As linhas principais máximas de $\mathbf{S}_{\varepsilon,-}$ são translações paralelas das linhas principais mínimas de $\mathbf{S}$.

Veja figuras 4.3 e 4.4. onde temos contato cúbico e figura 4.2 onde o contato é quadrático.

O tubo $\mathbf{T}_{\varepsilon}$ justapõe-se à superfície $\mathbf{S}_{\varepsilon,+}$ em $\theta=0$ e à superfície $\mathbf{S}_{\varepsilon,-}$ em $\theta=\pi$. O tubo está orientado positivamente por sua normal exterior e a superfície $\mathbf{S}_{\varepsilon,+}$ tem orientação oposta à da superfície $\mathbf{S}_{\varepsilon,-}$. As linhas principais mínimas de $\mathbf{S}_{\varepsilon,-}$ são obtidas por deslocamentos paralelos das linhas principais máximas de $\mathbf{S}_{\varepsilon,+}$ e as linhas principais máximas de $\mathbf{S}_{\varepsilon,-}$ são obtidas por deslocamentos paralelos das linhas principais mínimas de $\mathbf{S}_{\varepsilon,+}$.

A definição 4.2.6 permite-nos considerar a configuração principal da superfície $\mathbf{S}_{\varepsilon}=\mathbf{S}_{\varepsilon,+} \cup \mathbf{T}_{\varepsilon} \cup \mathbf{S}_{\varepsilon,-}$ como segue: 

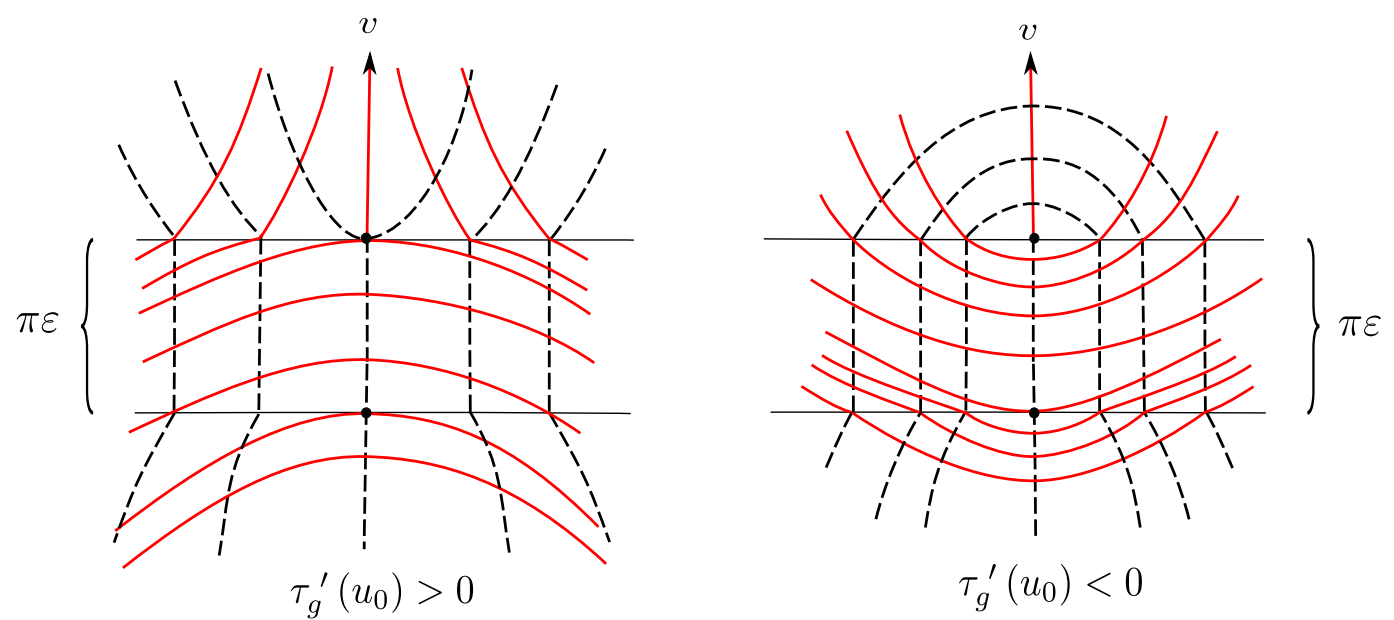

Figura 4.2: Configurações suaves por partes da superfície $\mathbf{S}_{\varepsilon}=\mathbf{S}_{\varepsilon,+} \cup \mathbf{T}_{\varepsilon} \cup \mathbf{S}_{\varepsilon,-}$ em uma vizinhança do ponto de tangência quadrática, sendo $k_{n}\left(u_{0}\right)<k_{n}^{\perp}\left(u_{0}\right)$. Dois casos: tangência interna se $\tau_{g}^{\prime}\left(u_{0}\right)>0$, à esquerda, e tangência interna se $\tau_{g}^{\prime}\left(u_{0}\right)<0$, à direita. Para mais detalhes sobre o caso quadrático ver [5].

Definição 4.2.7. A configuração principal da superfície $\mathbf{S}_{\varepsilon}=\mathbf{S}_{\varepsilon,+} \cup \mathbf{T}_{\varepsilon} \cup \mathbf{S}_{\varepsilon,-}$ consiste de um par de folheações seccionalmente suaves, sendo suas singularidades os pontos umbílicos de $\mathbf{S}_{\varepsilon} \backslash \mathbf{B}_{\varepsilon}$ e as tangências minimas (respectivamente máximas) destas folhas $\operatorname{com} \mathbf{B}_{\varepsilon}$ quando $\varepsilon>0$ (respectivamente $\varepsilon<0$ ). As folhas suaves por partes são definidas pela justaposição das linhas de curvatura principal de $\mathbf{S}_{\varepsilon,+}, \mathbf{T}_{\varepsilon}$ e $\mathbf{S}_{\varepsilon,-}$ como na definição 4.2.6.

Na figura 4.3, abaixo, em $u=u_{0}$ considerando $\tau_{g}\left(u_{0}\right)=\tau_{g}^{\prime}\left(u_{0}\right)=0, \tau_{g}^{\prime \prime}\left(u_{0}\right) \neq 0 \mathrm{e}$ $k_{n}\left(u_{0}\right)<k_{n}^{\perp}\left(u_{0}\right)$, o contato entre a linha de curvatura principal mínima da superfície $\mathbf{S}_{\varepsilon,+}$ (linha tracejada) e o bordo comum $\mathbf{B}_{\varepsilon,+}$ é cúbico (veja proposição 4.2.2). No semitubo de raio $\varepsilon$ e centro variando sobre $\mathbf{B}$ temos que a linha principal máxima (linha contínua) tem contato cúbico $\operatorname{com} \mathbf{B}_{\varepsilon,+}$ em $\theta=0$ e com $\mathbf{B}_{\varepsilon,-}$ em $\theta=\pi$ (veja proposição 4.2.5). Para a superfície $\mathbf{S}_{\varepsilon,-}$, o contato cúbico é entre a linha de curvatura principal máxima (linha contínua) e o bordo comum $\mathbf{B}_{\varepsilon,-}$.

Na figura 4.4, abaixo, em $u=u_{0}$ temos que $\tau_{g}\left(u_{0}\right)=\tau_{g}^{\prime}\left(u_{0}\right)=0, \tau_{g}^{\prime \prime}\left(u_{0}\right) \neq 0$, $k_{n}\left(u_{0}\right)>k_{n}^{\perp}\left(u_{0}\right)$ e as considerações são análogas às feitas para a figura 4.3 . 

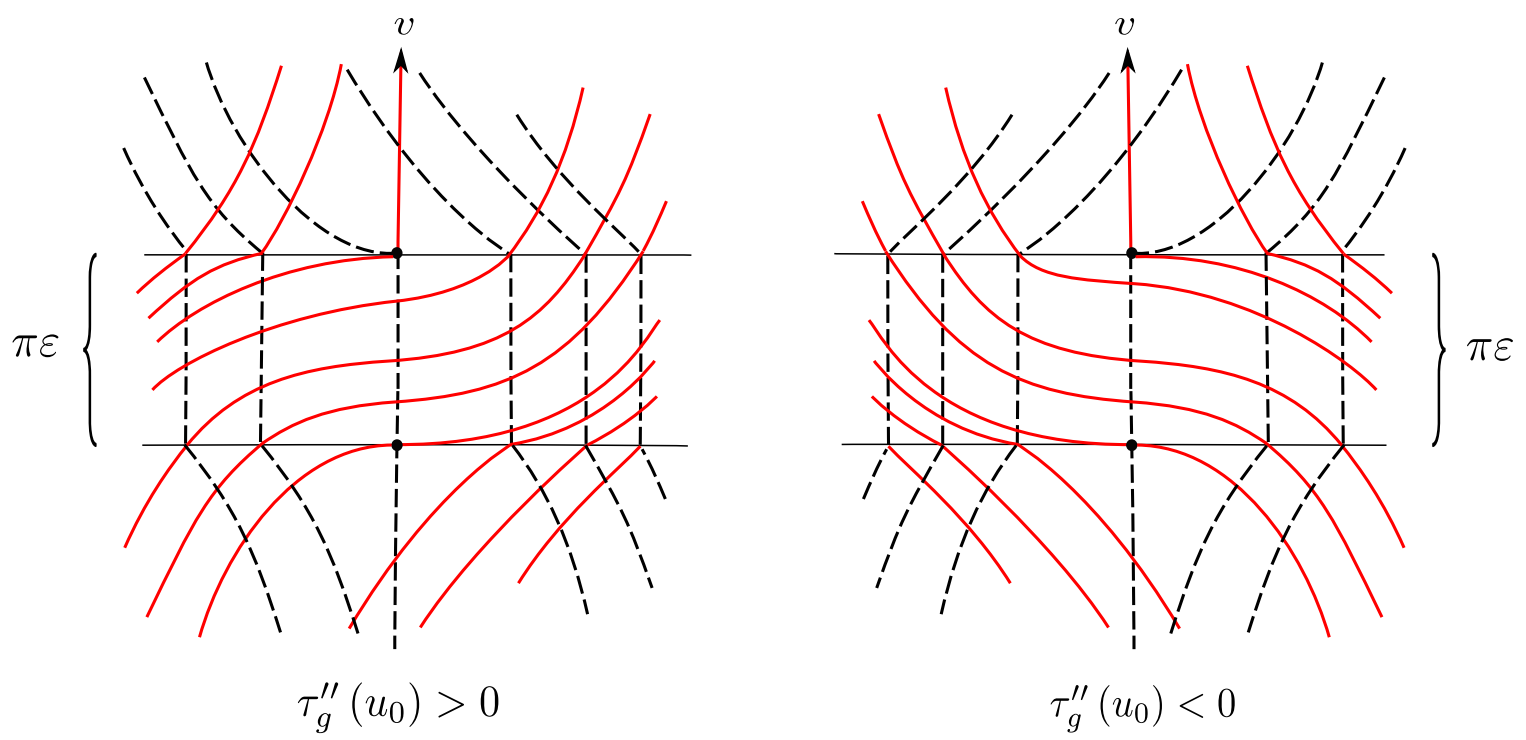

Figura 4.3: Configurações suaves por partes da superfície $\mathbf{S}_{\varepsilon}=\mathbf{S}_{\varepsilon,+} \cup \mathbf{T}_{\varepsilon} \cup \mathbf{S}_{\varepsilon,-}$ em uma vizinhança do ponto de contato cúbico, sendo $k_{n}\left(u_{0}\right)<k_{n}^{\perp}\left(u_{0}\right)$. Dois casos: $\tau_{g}^{\prime \prime}\left(u_{0}\right)>0$, à esquerda e $\tau_{g}^{\prime \prime}\left(u_{0}\right)<0$, à direita.
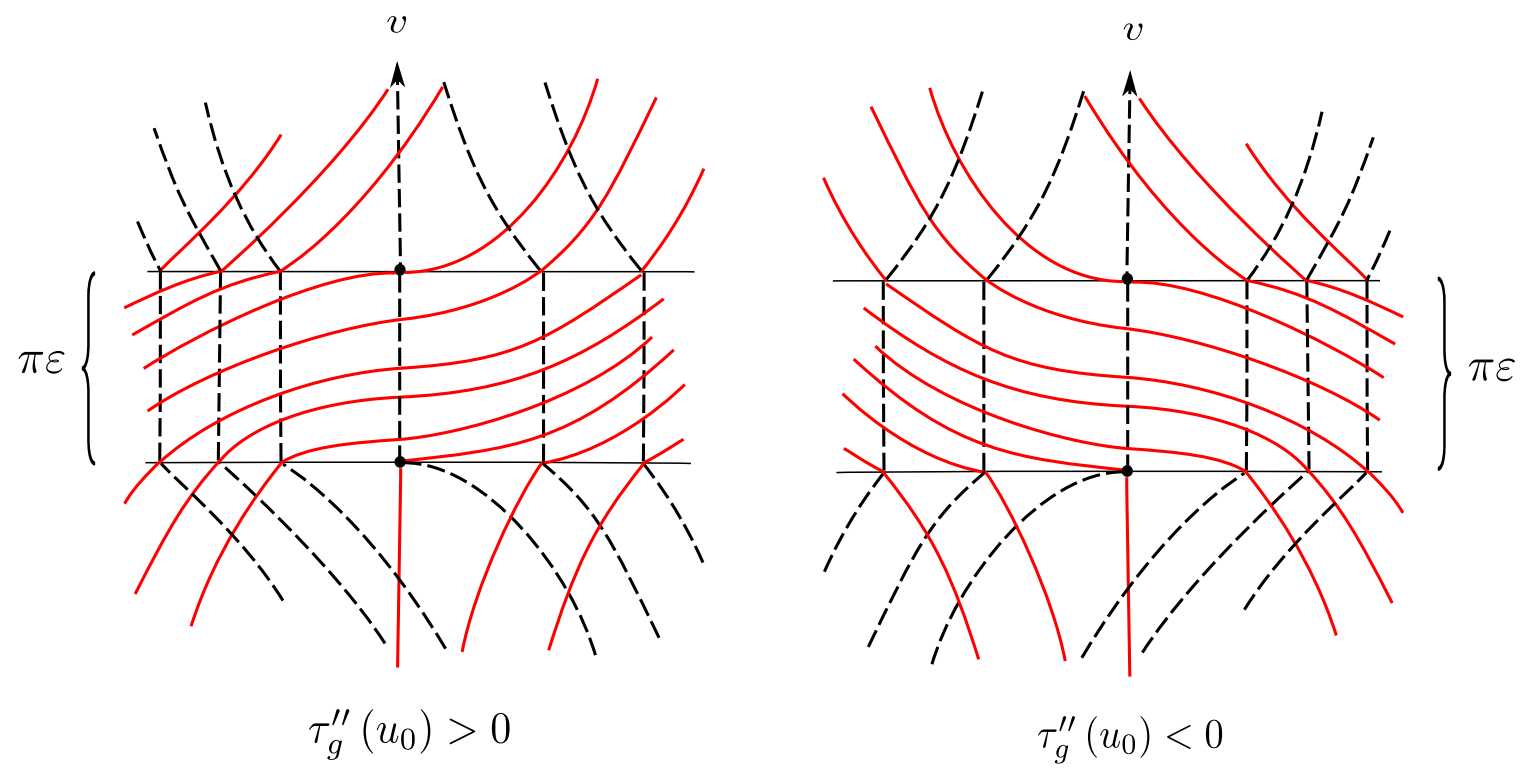

Figura 4.4: Configuraçôes suaves por partes da superfície $\mathbf{S}_{\varepsilon}=\mathbf{S}_{\varepsilon,+} \cup \mathbf{T}_{\varepsilon} \cup \mathbf{S}_{\varepsilon,-}$ em uma vizinhança do ponto de contato cúbico, sendo $k_{n}\left(u_{0}\right)>k_{n}^{\perp}\left(u_{0}\right)$. Dois casos: $\tau_{g}^{\prime \prime}\left(u_{0}\right)>0$, ̀̀ esquerda e $\tau_{g}^{\prime \prime}\left(u_{0}\right)<0$, à direita.

\subsection{Regularização de $\mathrm{S}_{\varepsilon}$ em uma Carta Local}

Nesta seção vamos estudar as configurações principais das $\mu$-regularizações das superfícies $\mathbf{S}_{\varepsilon}$, para $\varepsilon, \mu>0$ suficientemente pequenos.

Vamos supor que $\delta$ é uma parametrização regular de uma componente conexa de $\mathbf{B}$ e que $\delta_{+}$é uma parametrização pelo comprimento de arco $u$ da curva $\mathbf{B}_{\varepsilon,+}$ tal que $\{T, B=\mathcal{N} \wedge T, \mathcal{N}\}$ é um referencial móvel de $\mathbb{R}^{3}$ ao longo de $\delta_{+}$. Portanto, $\mathbf{B}=\mathbf{B}_{\varepsilon,+}-\varepsilon \mathcal{N}$ e $\delta=\delta_{+}-\varepsilon \mathcal{N}$. 
Na proposição 4.3.1, abaixo, apresentamos uma parametrização para o tubo de raio constante $\varepsilon>0$ e centro sobre a cuva $\delta_{+}-\varepsilon \mathcal{N}$. Este tubo é o envelope da família de esferas de raio $\varepsilon>0$ e centro variando sobre $\delta_{+}-\varepsilon \mathcal{N}$, veja exemplo 4.1 .1

Proposição 4.3.1. Seja $\delta_{+}:[0, l] \longrightarrow \mathbb{R}^{3}$ uma parametrização pelo comprimento de arco $u$ de uma componente conexa de $\mathbf{B}_{\varepsilon,+}$ tal que $\{T, B=\mathcal{N} \wedge T, \mathcal{N}\}$ é um referencial móvel de $\mathbb{R}^{3}$ ao longo de $\delta_{+}$. Então a expressão

$$
\begin{aligned}
Y_{\varepsilon}(u, v) & =\delta_{+}(u)-\left\{\frac{\varepsilon^{2}\left[1+\varepsilon k_{n}(u)\right] \tau_{g}(u) \operatorname{sen}(2 v)}{\left[1+\varepsilon k_{n}(u)\right]^{2}-\varepsilon^{2} \tau_{g}(u)[1+\operatorname{sen} v]}\right\} T(u)+ \\
& +\left\{\frac{\varepsilon\left[1+\varepsilon k_{n}(u)\right]^{2} \operatorname{sen}(2 v)}{\left[1+\varepsilon k_{n}(u)\right]^{2}-\varepsilon^{2} \tau_{g}(u)[1+\operatorname{sen} v]}\right\} B(u)- \\
& -\left\{\frac{2 \varepsilon\left[1+\varepsilon k_{n}(u)\right]^{2} \operatorname{sen}^{2} v}{\left[1+\varepsilon k_{n}(u)\right]^{2}-\varepsilon^{2} \tau_{g}(u)[1+\operatorname{sen} v]}\right\} \mathcal{N}(u), \quad v \in[0,2 \pi]
\end{aligned}
$$

cuja expansão em série de Taylor nas variáveis $v$ e $\varepsilon$, até a ordem 5 , na vizinhança de $v=\varepsilon=0$ é dada por:

$$
\begin{aligned}
Y_{\varepsilon}(u, v) & =\delta_{+}(u)+ \\
& +\left\{\tau_{g}(u)\left[2 \varepsilon^{2} \tau_{g}^{2}(u)+2 \varepsilon k_{n}(u)-2 \varepsilon^{2} k_{n}^{2}(u)-2\right] \varepsilon^{2} v+\frac{4}{3} \tau_{g}(u) \varepsilon^{2} v^{3}+O(6)\right\} T(u)+ \\
& +\left\{2\left[1-\varepsilon^{2} \tau_{g}^{2}(u)\left(1-2 \varepsilon k_{n}(u)\right)\right] \varepsilon v-\frac{4}{3} \varepsilon v^{3}+O(6)\right\} B(u)+ \\
& +\left\{2\left[\varepsilon^{2} \tau_{g}^{2}(u)-1\right] \varepsilon v^{2}+\frac{2}{3} \varepsilon v^{4}+O(6)\right\} \mathcal{N}(u)
\end{aligned}
$$

define uma parametrização regular do tubo de raio $\varepsilon$ e centro variando ao longo de $\delta_{+}-\varepsilon \mathcal{N}$, onde $k_{n}(u)$ é a curvatura normal e $\tau_{g}(u)$ é a torção geodésica da curva bordo, $\mathbf{B}_{\varepsilon,+}$, da superfície $\mathbf{S}_{\varepsilon,+}$. Veja figura 4.5 .

Demonstração. Denotemos por $\mathbf{T}_{\varepsilon}$ o tubo de raio $\varepsilon$ e centro variando sobre $\delta_{+}-\varepsilon \mathcal{N}$. Como $\mathbf{T}_{\varepsilon}$ é o envelope da família de esferas de raio $\varepsilon$ e centro sobre $\delta_{+}-\varepsilon \mathcal{N}$ temos que $P \in \mathbf{T}_{\varepsilon}$ satisfaz:

$$
\left\|P-\left(\delta_{+}(u)-\varepsilon \mathcal{N}(u)\right)\right\|^{2}=\varepsilon^{2} .
$$

Derivando a equação 4.9 em relação à variável $u$ obtemos

$$
\left\langle P-\delta_{+}(u)+\varepsilon \mathcal{N}(u),-\delta_{+}^{\prime}(u)+\varepsilon \mathcal{N}^{\prime}(u)\right\rangle=0
$$



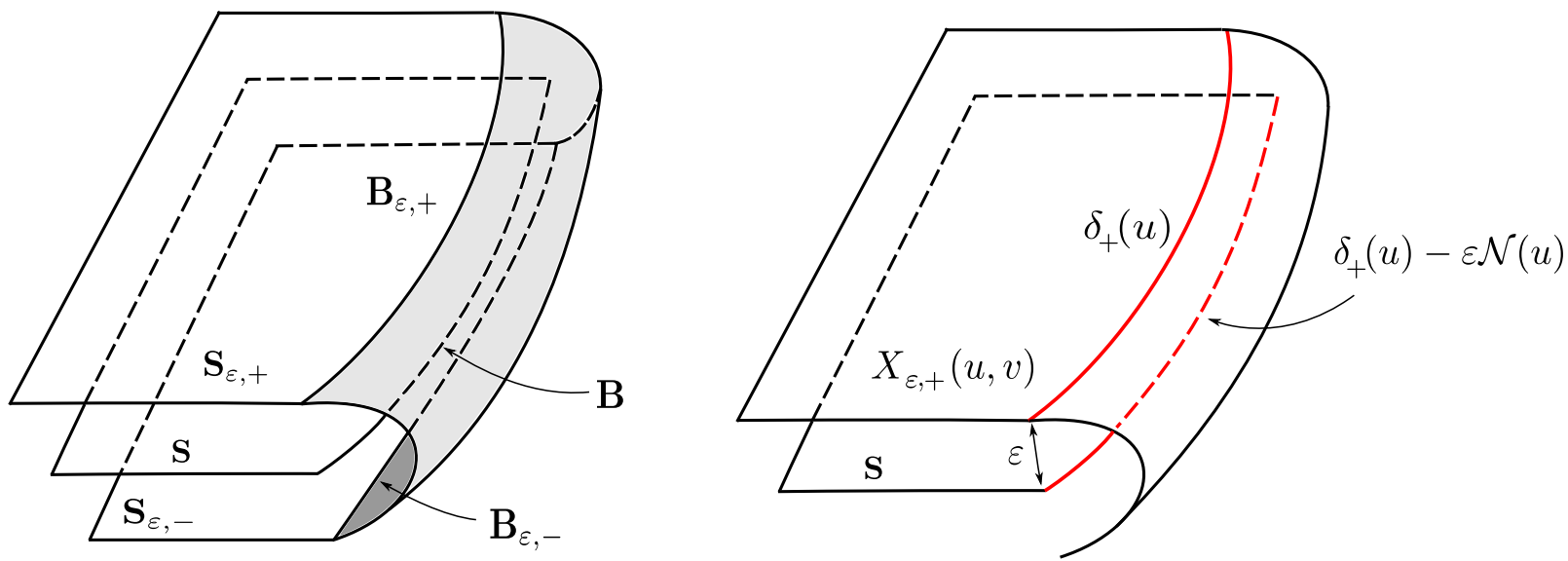

Figura 4.5: $\mathbf{B}$ é o deslocamento paralelo do bordo $\mathbf{B}_{\varepsilon,+}$ na direção de $-\mathcal{N}$, sendo $\mathcal{N}$ campo normal da superfície $\mathbf{S}_{\varepsilon,+}$.

Observe que o sistema de equações

$$
\left\{\begin{aligned}
\left\|P-\left(\delta_{+}(u)-\varepsilon \mathcal{N}(u)\right)\right\|^{2} & =\varepsilon^{2} \\
\left\langle P-\left(\delta_{+}(u)-\varepsilon \mathcal{N}(u)\right),-\delta_{+}^{\prime}(u)+\varepsilon \mathcal{N}^{\prime}(u)\right\rangle & =0
\end{aligned}\right.
$$

representa um círculo na esfera de raio $\varepsilon$ e centro sobre a curva $\delta_{+}-\varepsilon \mathcal{N}$ pois, para cada $u$ fixado, tal sistema define a interseção da esfera e do cone de vértice $\delta_{+}(u)-\varepsilon \mathcal{N}(u)$.

Lembremos que o referencial de Darboux $\{T, B=\mathcal{N} \wedge T, \mathcal{N}\}$ ao longo de $\delta_{+}$satisfaz o seguinte sistema de equações diferenciais:

$$
\begin{gathered}
T^{\prime}(u)=k_{g}(u) B(u)+k_{n}(u) \mathcal{N}(u), \\
B^{\prime}(u)=-k_{g}(u) T(u)+\tau_{g}(u) \mathcal{N}(u), \\
\mathcal{N}^{\prime}(u)=-k_{n}(u) T(u)-\tau_{g}(u) B(u),
\end{gathered}
$$

onde $k_{n}(u)$ é a curvatura normal, $k_{g}(u)$ é a curvatura geodésica e $\tau_{g}(u)$ é a torção geodésica da curva bordo deslocada $\mathbf{B}_{\varepsilon,+}$.

Assim, supondo que $P=\delta_{+}(u)+x T(u)+y B(u)+z \mathcal{N}(u)$ nas equações (4.11), obtemos

$$
\left\{\begin{aligned}
x^{2}+y^{2}+(z+\varepsilon)^{2} & =\varepsilon^{2} \\
\left\langle x T(u)+y B(u)+(z+\varepsilon) \mathcal{N}(u),-\delta_{+}^{\prime}(u)+\varepsilon \mathcal{N}^{\prime}(u)\right\rangle & =0
\end{aligned}\right. \text {, }
$$

o qual pode ser reescrito, usando o referencial de Darboux ao longo de $\mathbf{B}_{\varepsilon,+}$, da seguinte forma:

$$
\left\{\begin{aligned}
x^{2}+y^{2}+(z+\varepsilon)^{2} & =\varepsilon^{2} \\
\left\langle x T(u)+y B(u)+(z+\varepsilon) \mathcal{N}(u),-T(u)+\varepsilon\left[-k_{n}(u) T(u)-\tau_{g}(u) B(u)\right]\right\rangle & =0
\end{aligned}\right. \text {. }
$$


O sistema 4.13 reduz-se a

$$
\left\{\begin{array}{rll}
x^{2}+y^{2}+(z+\varepsilon)^{2} & = & \varepsilon^{2} \\
\left(1+\varepsilon k_{n}(u)\right) x+\varepsilon \tau_{g}(u) y & = & 0
\end{array} .\right.
$$

Como já foi discutido acima, para cada $u$ fixado, o sistema (4.14) representa um círculo de raio $f(u, v)$ na esfera de raio $\varepsilon$ e centro sobre a curva $\delta_{+}(u)-\varepsilon \mathcal{N}(u)$, considerando $v>0$ como sendo o ângulo indicado na figura 4.6, abaixo.

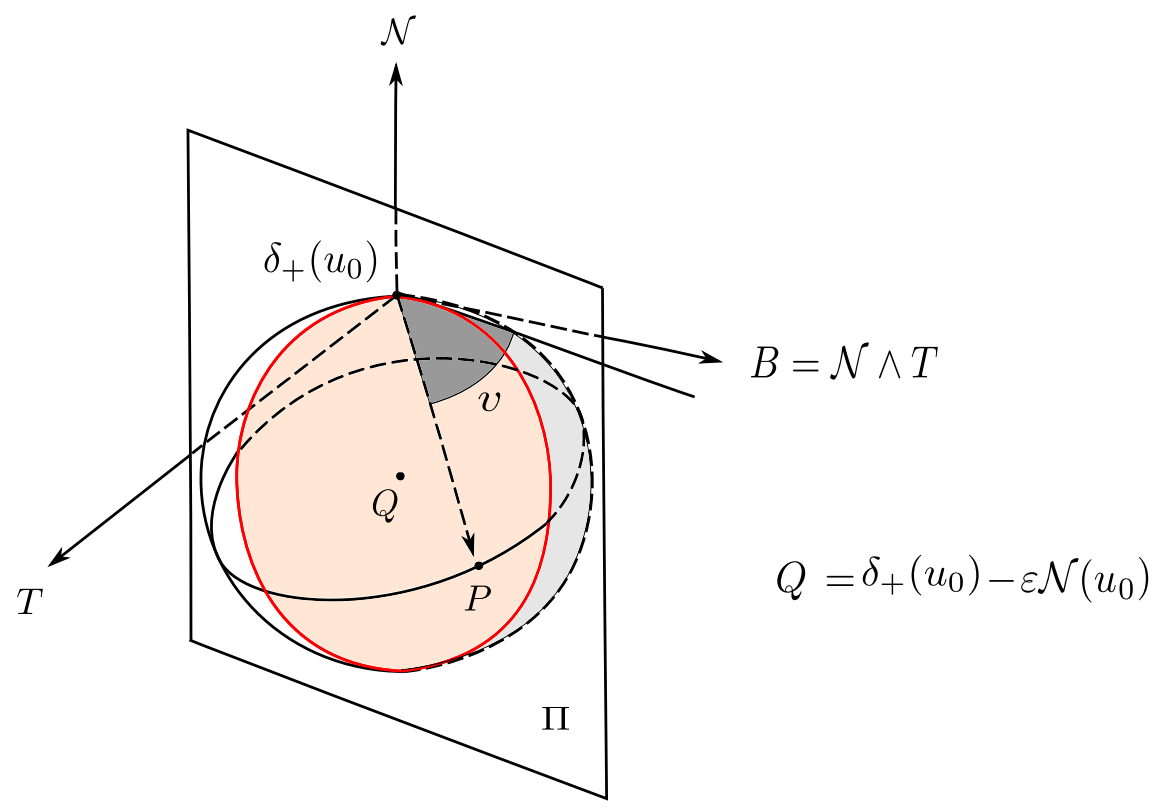

Figura 4.6: Solução do sistema (4.14): para cada $u_{0}$ fixado temos um círculo de raio $f\left(u_{0}, v\right)$ obtido da interseção entre a esfera de centro $Q=\delta_{+}\left(u_{0}\right)-\varepsilon \mathcal{N}\left(u_{0}\right)$ e raio $\varepsilon$ e o plano $\Pi$ de equação $\left(1+\varepsilon k_{n}\left(u_{0}\right)\right) x+\varepsilon \tau_{g}\left(u_{0}\right) y=0$.

Portanto, sendo $y=f(u, v) \cos v$ e $z=-f(u, v) \operatorname{sen} v$ 11 no sistema (4.14, obtemos:

$$
\left\{\begin{aligned}
x^{2}+f^{2}(u, v)-2 \varepsilon f(u, v) \operatorname{sen} v & =0 \\
\left(1+\varepsilon k_{n}(u)\right) x+\varepsilon \tau_{g}(u) f(u, v) \cos v & =0
\end{aligned}\right. \text {. }
$$

A solução não nula de (4.15) é dada por:

$$
\begin{gathered}
x=-\frac{2 \varepsilon^{2}\left[1+\varepsilon k_{n}(u)\right] \tau_{g}(u) \operatorname{sen} v \cos v}{\left[1+\varepsilon k_{n}(u)\right]^{2}-\varepsilon^{2} \tau_{g}(u)(1+\operatorname{sen} v)}, \\
f(u, v)=\frac{2 \varepsilon\left[1+\varepsilon k_{n}(u)\right]^{2} \operatorname{sen} v}{\left[1+\varepsilon k_{n}(u)\right]^{2}-\varepsilon^{2} \tau_{g}(u)(1+\operatorname{sen} v)} .
\end{gathered}
$$

Substituindo os valores obtidos acima para $x, y=f(u, v) \cos v$ e $z=-f(u, v) \operatorname{sen} v$,

\footnotetext{
${ }^{1}$ Consideramos $z=-f(u, v)$ sen $v$, pois como $v \in[0,-2 \pi],-v \in[0,2 \pi]$ e $\operatorname{sen}(-v)=-\operatorname{sen} v$.
} 
obtemos

$$
\begin{aligned}
P & =\delta_{+}(u)+x T(u)+y B(u)+z \mathcal{N}(u) \\
& =\delta_{+}(u)-\left\{\frac{\varepsilon^{2}\left[1+\varepsilon k_{n}(u)\right] \tau_{g}(u) \operatorname{sen}(2 v)}{\left[1+\varepsilon k_{n}(u)\right]^{2}-\varepsilon^{2} \tau_{g}(u)(1+\operatorname{sen} v)}\right\} T(u)+ \\
& +\left\{\frac{\varepsilon\left[1+\varepsilon k_{n}(u)\right]^{2} \operatorname{sen}(2 v)}{\left[1+\varepsilon k_{n}(u)\right]^{2}-\varepsilon^{2} \tau_{g}(u)(1+\operatorname{sen} v)}\right\} B(u)-\left\{\frac{2 \varepsilon\left[1+\varepsilon k_{n}(u)\right]^{2} \operatorname{sen}^{2} v}{\left[1+\varepsilon k_{n}(u)\right]^{2}-\varepsilon^{2} \tau_{g}(u)(1+\operatorname{sen} v)}\right\} \mathcal{N}(u),
\end{aligned}
$$

cuja expansão em série de Taylor nas variáveis $v$ e $\varepsilon$, até a ordem 5 , na vizinhança de $v=\varepsilon=0$ é dada por:

$$
\begin{aligned}
Y_{\varepsilon}(u, v) & =\delta_{+}(u)+ \\
& +\left\{\tau_{g}(u)\left[2 \varepsilon^{2} \tau_{g}^{2}(u)+2 \varepsilon k_{n}(u)-2 \varepsilon^{2} k_{n}^{2}(u)-2\right] \varepsilon^{2} v+\frac{4}{3} \tau_{g}(u) \varepsilon^{2} v^{3}+O(6)\right\} T(u)+ \\
& +\left\{2\left[1-\varepsilon^{2} \tau_{g}^{2}(u)\left(1-2 \varepsilon k_{n}(u)\right)\right] \varepsilon v-\frac{4}{3} \varepsilon v^{3}+O(6)\right\} B(u)+ \\
& +\left\{2\left[\varepsilon^{2} \tau_{g}^{2}(u)-1\right] \varepsilon v^{2}+\frac{2}{3} \varepsilon v^{4}+O(6)\right\} \mathcal{N}(u)
\end{aligned}
$$

Isto completa a demonstração.

Proposição 4.3.2. Seja $\delta_{+}:[0, l] \longrightarrow \mathbb{R}^{3}$ uma parametrização pelo comprimento de arco $u$ de uma componente conexa de $\mathbf{B}_{\varepsilon,+}$ tal que $\{T, B, \mathcal{N}\}$ é um referencial móvel de $\mathbb{R}^{3}$ ao longo de $\delta_{+}$. Então as expressões

$$
\begin{aligned}
X_{\varepsilon,+}(u, v) & =\delta_{+}(u)+v B(u)+\left[k_{n}^{\perp}(u) \frac{v^{2}}{2}+A(u, \varepsilon) \frac{v^{3}}{6}+C(u, v, \varepsilon) v^{3}\right] \mathcal{N}(u),-\mu<v \leq 0 \\
Y_{\varepsilon}(u, v) & =\delta_{+}(u)+\left\{\tau_{g}(u)\left[\varepsilon^{2} \tau_{g}^{2}(u)+\varepsilon k_{n}(u)-\varepsilon^{2} k_{n}^{2}(u)-1\right] \varepsilon v+\frac{\tau_{g}(u)}{6 \varepsilon} v^{3}+O(6)\right\} T(u)+ \\
& +\left\{\left[1-\varepsilon^{2} \tau_{g}^{2}(u)\left(1-2 \varepsilon k_{n}(u)\right)\right] v-\frac{v^{3}}{6 \varepsilon^{2}}+O(6)\right\} B(u)+ \\
& +\left\{\frac{1}{2}\left[-1+\varepsilon^{2} \tau_{g}^{2}(u)\right] \frac{v^{2}}{\varepsilon}+\frac{v^{4}}{24 \varepsilon^{3}}+O(6)\right\} \mathcal{N}(u), \quad 0<v<\mu
\end{aligned}
$$

definem uma parametrização regular de classe $C^{1}$ para a superfície $\mathbf{S}_{\varepsilon}$, onde $C(u, 0, \varepsilon)=0$, $k_{n}^{\perp}$ é a curvatura normal da superfície $\mathbf{S}_{\varepsilon,+}$ na direção de $B=\mathcal{N} \wedge T, k_{n}$ e $\tau_{g}$ são, 
respectivamente, a curvatura normal e a torção geodésica da curva bordo deslocada $\mathbf{B}_{\varepsilon,+}$. Demonstração. Observe que a parte linear de $Y_{\varepsilon}$ em (4.8), proposição 4.3.1, é dada por $Y_{\varepsilon}(u, v)=\delta_{+}(u)+2 \varepsilon v B(u)$ e a parte linear de $X_{\varepsilon,+}$ em 4.17 dada por $X_{\varepsilon,+}=\delta_{+}(u)+v B(u)$. Portanto, considerando o reescalonamento

$$
v=\frac{\widetilde{v}}{2 \varepsilon}
$$

em (4.8) e reescrevendo $\widetilde{v}=v\left(\varepsilon>0\right.$ suficientemente pequeno), obtemos a expressão de $Y_{\varepsilon}$ em (4.17) e garantimos que as parametrizações em (4.17) da superfície, $X_{\varepsilon,+}$, e do tubo, $Y_{\varepsilon}$, ao longo da curva $\delta_{+}$são $C^{1}$ - próximas.

A demostração segue análoga à da proposição 3.1.1 do capítulo 3.

Uma parametrização da $\mu$-regularização da superfície $\mathbf{S}_{\varepsilon}$ será apresentada na próxima definição 4.3.3. na qual a função de transição $\phi: \mathbb{R} \longrightarrow \mathbb{R}$ é (como na definição 3.2.1 do capítulo 3) decrescente em $[0, \mu], \phi(t)=0$, para $t \geq \mu, \phi(t)=1$, para $t \leq 0 \mathrm{e}$ $\phi^{(n)}(0)=\phi^{(n)}(\mu)=0$, para todo $n \in \mathbb{N}$. Veja no apêndice 4.4 um exemplo de uma tal $\phi$.

Definição 4.3.3. [Regularização] Sejam $\phi$ uma função de transição e a superfície $\mathbf{S}_{\varepsilon}=\mathbf{S}_{\varepsilon,+} \cup \mathbf{T}_{\varepsilon} \cup \mathbf{S}_{\varepsilon,-}$ como definida na seção 4.2. Para $\mu>0$, a $\mu$-regularização de $\mathbf{S}_{\varepsilon}$, denotada por $\mathbf{S}_{\varepsilon, \mu}$ é (não unicamente) definida por

$$
Z(u, v, \varepsilon)=\phi\left(\frac{v}{\varepsilon^{2}}\right) X_{\varepsilon,+}(u, v)+\left[1-\phi\left(\frac{v}{\varepsilon^{2}}\right)\right] Y_{\varepsilon}(u, v), \quad-\varepsilon^{2} \mu<v<\varepsilon^{2} \mu,
$$

onde $X_{\varepsilon,+}$ e $Y_{\varepsilon}$ são, respectivamente, as parametrizações da superfície $\mathbf{S}_{\varepsilon,+}$ e do tubo $\mathbf{T}_{\varepsilon}$ dadas pelas expressões 4.17) da proposição 4.3.2.

Assim, a equação 4.18 torna-se:

$$
\begin{aligned}
& Z(u, v, \varepsilon)=\delta_{+}(u)-\left\{\tau_{g}(u) \varepsilon v-\tau_{g}(u) k_{n}(u) \varepsilon^{2} v-\phi\left(\frac{v}{\varepsilon^{2}}\right)\left[1-\varepsilon k_{n}(u)\right] \tau_{g}(u) \varepsilon v\right\} T(u)+ \\
&+\left\{v-\tau_{g}^{2}(u) v \varepsilon^{2}-\frac{1}{6} \frac{v^{3}}{\varepsilon^{2}}+\phi\left(\frac{v}{\varepsilon^{2}}\right)\left[\frac{v^{2}}{6 \varepsilon^{2}}+\varepsilon^{2} \tau_{g}^{2}(u)\right] v\right\} B(u)- \\
&-\left\{\frac{v^{2}}{2 \varepsilon}-\phi\left(\frac{v}{\varepsilon^{2}}\right)\left[\frac{1}{2 \varepsilon}+\frac{k_{n}^{\perp}(u)}{2} v+\frac{1}{6} A(u, \varepsilon) v+C(u, v, \varepsilon) v\right] v^{2}\right\} \mathcal{N}(u), \\
&-\varepsilon^{2} \mu<v<\varepsilon^{2} \mu,
\end{aligned}
$$

onde $C(u, 0, \varepsilon)=0$ e $\{T, B, \mathcal{N}\}$ é um referencial móvel de $\mathbb{R}^{3}$ ao longo de $\delta_{+}(u)$.

Façamos o reescalonamento $v=\varepsilon^{2} w$ na expressão 4.19 . Logo, na carta $(u, w)$ a 
parametrização de $\mathbf{S}_{\varepsilon, \mu}$ é dada por:

$$
\begin{aligned}
Z(u, w, \varepsilon) & =\delta_{+}(u)-\left\{\tau_{g}(u) \varepsilon^{3} w-\tau_{g}(u) k_{n}(u) \varepsilon^{4} w^{2}-\phi(w)\left[1-\varepsilon k_{n}(u)\right] \tau_{g}(u) \varepsilon^{3} w\right\} T(u)+ \\
& +\left\{\varepsilon^{2} w-\tau_{g}^{2}(u) \varepsilon^{4} w-\frac{\varepsilon^{4} w^{3}}{6}+\phi(w)\left[\frac{\varepsilon^{2} w^{2}}{6}+\varepsilon^{2} \tau_{g}^{2}(u)\right] \varepsilon^{2} w\right\} B(u)- \\
& -\left\{\frac{\varepsilon^{3} w^{2}}{2}-\phi(w)\left[\frac{1}{2 \varepsilon}+\frac{k_{n}^{\perp}(u)}{2} \varepsilon^{2} w+\frac{1}{6} A(u, \varepsilon) \varepsilon^{2} w+C(u, w, \varepsilon) \varepsilon^{4} w\right] \varepsilon^{4} w^{2}\right\} \mathcal{N}(u),
\end{aligned}
$$

$-\mu<w<\mu$, onde $C(u, 0, \varepsilon)=0$.

O nosso objetivo é estudar os pontos umbílicos Darbouxianos e suas transições sobre a superfície regularizada $\mathbf{S}_{\varepsilon, \mu}, \varepsilon, \mu>0$ suficientemente pequenos. Para isso, usando a parametrização dada em (4.20), vamos determinar a equação diferencial das linhas de curvatura na carta $(u, w)$, a qual é dada por:

$$
L(u, w, \varepsilon) d w^{2}+M(u, w, \varepsilon) d u d w+N(u, w, \varepsilon) d u^{2}=0
$$

sendo $L=F g-G f, M=E g-G e$ e $N=E f-F e$.

Abaixo, omitiremos a variável $u$ das funções $k_{n}^{\perp}(u), k_{n}(u)$ e $\tau_{g}(u)$ e a variável $w$ da função $\phi(w)$ a fim de simplificar a notação.

Considerando $Z_{\varepsilon}(u, w)=Z(u, w, \varepsilon)$, da equação 4.20 temos na carta $(u, w)$ :

$$
\begin{aligned}
\frac{\partial Z_{\varepsilon}}{\partial u} & =\left\{1-\varepsilon^{2} w k_{g}+\varepsilon^{3}\left[w(1-\phi)\left(\frac{1}{2} w k_{n}-\tau_{g}^{\prime}\right)\right]+\varepsilon^{4} w(\cdots)\right\} T(u)+ \\
& +\left\{\frac{1}{2} \varepsilon^{3} w \tau_{g}\left(w-2 k_{g}\right)(1-\phi)+\frac{1}{2} \varepsilon^{4} w \tau_{g}(\cdots)\right\} B(u)+ \\
& +\left\{\varepsilon^{2} w \tau_{g}-\varepsilon^{3} w \tau_{g} k_{n}(1-\phi)+\varepsilon^{4} w(\cdots)\right\} \mathcal{N}(u) \\
\frac{\partial Z_{\varepsilon}}{\partial w}= & \left\{\varepsilon^{3} \tau_{g}\left(w \phi^{\prime}-\phi\right)+\varepsilon^{4} k_{n} \tau_{g}\left(1-\phi-w \phi^{\prime}\right)+\varepsilon^{5}(\cdots)\right\} T(u)+ \\
+ & \left\{\varepsilon^{2}-\varepsilon^{4}\left[\tau_{g}^{2}\left(1-\phi-w \phi^{\prime}\right)+\frac{1}{2} w^{2}\left(1-\phi-\frac{1}{3} w \phi^{\prime}\right)\right]+\varepsilon^{5} \tau_{g}^{2} k_{n}(\cdots)\right\} B(u)+ \\
+ & \left\{\varepsilon^{3} w\left(-1+\phi+\frac{1}{2} w \phi^{\prime}\right)+\varepsilon^{4} w k_{n}^{\perp}\left(\phi+\frac{1}{2} w \phi^{\prime}\right)+\varepsilon^{5} w(\cdots)\right\} \mathcal{N}(u) .
\end{aligned}
$$

O vetor normal (não unitário) a $\mathbf{S}_{\varepsilon, \mu}$ é dado por:

$$
\mathcal{N}_{\varepsilon}(u, w)=\left(\left(Z_{\varepsilon}\right)_{u} \wedge\left(Z_{\varepsilon}\right)_{w}\right)(u, w)=n_{1} T(u)+n_{2} B(u)+n_{3} \mathcal{N}(u),
$$


onde

$$
\begin{aligned}
n_{1} & =-\varepsilon^{4} w \tau_{g}+\varepsilon^{5} w \tau_{g} k_{n}(1-\phi)+\varepsilon^{6}\left\{w \tau_{g}\left(2 \tau_{g}^{2}+k_{n}^{2}\right)(1-\phi)+w^{2}(\cdots)\right\}+\varepsilon^{7}(\cdots), \\
n_{2} & =\varepsilon^{3} w\left(1-\phi-\frac{1}{2} w \phi^{\prime}\right)+\varepsilon^{4} w k_{n}^{\perp}\left(-\phi-\frac{1}{2} w \phi^{\prime}\right)+\varepsilon^{5}\left\{2 \tau_{g}^{2} w(\phi-1)+\right. \\
& \left.+w^{2}\left[k_{g}(\phi-1)+\frac{3}{2} \tau_{g}^{2} \phi^{\prime}\right]+w^{3}(\cdots)\right\}+\varepsilon^{6} w(\cdots), \\
n_{3} & =\varepsilon^{2}+\varepsilon^{4}\left\{\tau_{g}^{2}(\phi-1)+w\left[\tau_{g}^{2} \phi^{\prime}-k_{g}\right]+\frac{1}{2} w^{2}\left(-1+\phi+\frac{1}{3} \phi^{\prime}\right)+w^{3}(\cdots)\right\}+\varepsilon^{5}(\cdots) .
\end{aligned}
$$

Os coeficientes da Primeira Forma Fundamental de $Z_{\varepsilon}$, expressos na carta $(u, w)$, são:

$$
\begin{aligned}
E(u, w, \varepsilon)=\left\langle\frac{\partial Z_{\varepsilon}}{\partial u}, \frac{\partial Z_{\varepsilon}}{\partial u}\right\rangle & =1-2 \varepsilon^{2} w k_{g}+\varepsilon^{3} w\left[(1-\phi)\left(w k_{n}-2 \tau_{g}^{\prime}\right)\right]+\varepsilon^{4}(\cdots), \\
F(u, w, \varepsilon)=\left\langle\frac{\partial Z_{\varepsilon}}{\partial u}, \frac{\partial Z_{\varepsilon}}{\partial w}\right\rangle & =\varepsilon^{3}\left[\tau_{g}\left(-1+\phi+w \phi^{\prime}\right)+\right. \\
& \left.+\varepsilon \tau_{g} k_{n}\left(1-\phi-w \phi^{\prime}\right)+\varepsilon^{2}(\cdots)\right] \\
G(u, w, \varepsilon)=\left\langle\frac{\partial Z_{\varepsilon}}{\partial w}, \frac{\partial Z_{\varepsilon}}{\partial w}\right\rangle & =\varepsilon^{4}\left\{1+\varepsilon^{2}\left[-\tau_{g}^{2}\left(1-\phi^{2}\right)+2 \tau_{g}^{2} w \phi \phi^{\prime}+\right.\right. \\
& \left.\left.+w^{2}\left(-1+\tau_{g}^{2}\left(\phi^{\prime}\right)^{2}+\phi^{2}\right)+\cdots\right]\right\} .
\end{aligned}
$$

Os coeficientes da Segunda Forma Fundamental de $Z_{\varepsilon}$, expressos na carta $(u, w)$, são dados por $\frac{e}{\left|\mathcal{N}_{\varepsilon}\right|}, \frac{f}{\left|\mathcal{N}_{\varepsilon}\right|}$ e $\frac{g}{\left|\mathcal{N}_{\varepsilon}\right|}$, onde $\mathcal{N}_{\varepsilon}$ é o vetor normal definido em 4.21 e

$$
\begin{aligned}
e(u, w, \varepsilon) & =\left\langle\frac{\partial^{2} Z_{\varepsilon}}{\partial u^{2}}, \mathcal{N}_{\varepsilon}\right\rangle(u, w, \varepsilon)=\varepsilon^{2} k_{n}+\varepsilon^{3} k_{g} w\left(1-\phi-\frac{1}{2} w \phi^{\prime}\right)+\varepsilon^{4} w(\cdots), \\
f(u, w, \varepsilon) & =\left\langle\frac{\partial^{2} Z_{\varepsilon}}{\partial u \partial w}, \mathcal{N}_{\varepsilon}\right\rangle(u, w, \varepsilon)=\varepsilon^{4} \tau_{g}+\varepsilon^{5} \tau_{g} k_{n}\left(-1+\phi+w \phi^{\prime}\right)+ \\
& +\varepsilon^{6}\left\{\left(k_{n}^{\perp}\right)^{\prime} w\left(\phi+\frac{1}{2} w \phi^{\prime}\right)-2 \tau_{g}^{3}\left(1-\phi-w \phi^{\prime}\right)+\right. \\
& \left.+\tau_{g}\left[\frac{1}{2} k_{g} w\left(1-\phi-w \phi^{\prime}\right)\left(-2+2 \phi+w \phi^{\prime}\right)+k_{n}^{2}\left(1-\phi-w \phi^{\prime}\right)+w^{2}(\cdots)\right]\right\}
\end{aligned}
$$




$$
\begin{aligned}
g(u, w, \varepsilon) & =\left\langle\frac{\partial^{2} Z_{\varepsilon}}{\partial w^{2}}, \mathcal{N}_{\varepsilon}\right\rangle(u, w, \varepsilon)=\varepsilon^{5}\left[-1+\phi+2 w \phi^{\prime}+\frac{1}{2} w^{2} \phi^{\prime \prime}\right]+ \\
& +\varepsilon^{6} k_{n}^{\perp}\left[\phi+2 w \phi^{\prime}+\frac{1}{2} w^{2} \phi^{\prime \prime}\right]+\varepsilon^{7}(\cdots) .
\end{aligned}
$$

Sendo $L=F g-G f, \quad M=E g-G e$ e $N=E f-F e$, dos cálculos acima obtemos:

$$
\begin{aligned}
L(u, w, \varepsilon) & =-\varepsilon^{8} \tau_{g}(u)\left\{2 \phi(w)-\phi(w)^{2}+3 w \phi^{\prime}(w)(1-\phi(w))+\frac{1}{2} w^{2}\left[\phi^{\prime \prime}(w)(1-\phi(w))\right.\right. \\
& \left.\left.-4\left(\phi^{\prime}(w)\right)^{2}\right]-\frac{1}{2} w^{3} \phi^{\prime}(w) \phi^{\prime \prime}(w)\right\}+\varepsilon^{9} \tau_{g}(u)\left(k_{n}^{\perp}-k_{n}\right)(u)\{\phi(w)(\phi(w)-1)+ \\
& \left.+w \phi^{\prime}(w)(3 \phi(w)-2)-\frac{1}{2} w^{2}\left[\phi^{\prime \prime}(w)(\phi(w)-1)-4\left(\phi^{\prime}(w)\right)^{2}\right]+\frac{w^{3}}{2} \phi^{\prime} \phi^{\prime \prime}\right\}+ \\
& +\varepsilon^{10}(\cdots) \\
M(u, w, \varepsilon) & =\varepsilon^{5}\left\{-1+\phi(w)+2 w \phi^{\prime}(w)+\frac{1}{2} w^{2} \phi^{\prime \prime}(w)\right\}+ \\
& +\varepsilon^{6}\left\{k_{n}^{\perp}(u)-k_{n}(u)+k_{n}^{\perp}(u)\left[-1+\phi(w)+2 w \phi^{\prime}(w)+\frac{1}{2} w^{2} \phi^{\prime \prime}(w)\right]\right\}+\varepsilon^{7}(\cdots) \\
N(u, w, \varepsilon) & =\varepsilon^{4} \tau_{g}(u)+\varepsilon^{6}\left\{w\left(k_{n}^{\perp}(u)\right)^{\prime}\left[\phi(w)+\frac{1}{2} w \phi^{\prime}(w)\right]+\right. \\
& +\tau_{g}(u)\left[\frac{1}{4} w^{4}\left(\phi^{\prime}(w)\right)^{2}-\frac{2}{3} w^{3} \phi^{\prime}(w)+w^{3} \phi(w) \phi^{\prime}(w)-w^{2} \phi(w)+\right. \\
& \left.\left.+w^{2} \phi^{2}-2 w k_{g}(u)+2 w \tau_{g}^{2} \phi^{\prime}-2 \tau_{g}^{2}(\phi(w)-1)\right]\right\}+\varepsilon^{7} \tau_{g}(\cdots)
\end{aligned}
$$

O teorema 4.3.4 abaixo, é o principal resultado deste capítulo.

Teorema 4.3.4. Seja $\delta\left(u_{0}\right)$ um ponto de contato cúbico para a superfície $\mathbf{S}$ tal que $\tau_{g}\left(u_{0}\right)=0, \tau_{g}^{\prime}\left(u_{0}\right)=0, \tau_{g}^{\prime \prime}\left(u_{0}\right)>0 e\left(k_{n}^{\perp}-k_{n}\right)\left(u_{0}\right)<0$. Suponha que $k_{n}\left(u_{0}\right), k_{n}^{\prime}\left(u_{0}\right)$, $k_{n}^{\perp}\left(u_{0}\right),\left(k_{n}^{\perp}\right)^{\prime}\left(u_{0}\right),\left(k_{n}^{\perp}\right)^{\prime \prime}\left(u_{0}\right)$ são todos não nulos e que $k_{n}^{\prime}\left(u_{0}\right) \neq\left(k_{n}^{\perp}\right)^{\prime}\left(u_{0}\right)$.

Considere a família a 1 parâmetro de superfícies $\mathbf{S}_{\tau_{0}}$ com bordo variável $\mathbf{B}_{\tau_{0}}$, sendo $\tau_{0}$ definido pela expressão

$$
\tau_{g}(u)=\tau_{0}+\frac{\tau_{g}^{\prime \prime}(0)}{2} u^{2}+\frac{\tau_{g}^{\prime \prime \prime}(0)}{6} u^{3}+O(4),
$$

(ilustração na figura 4.7). Denotemos por $\mathbf{S}_{\tau_{0}, \varepsilon, \mu}$ o $\varepsilon$-engrossamento e $\mu$-regularização da superfície $\mathbf{S}_{\tau_{0}}$, $\varepsilon$, $\mu$ positivos e suficientemente pequenos (definição 4.3.3). No espaço de 
parâmetros $\varepsilon, \tau_{0}$ ( $\varepsilon, \tau_{0}$ suficientemente pequenos), existem duas curvas, $D_{23}$ e $D_{12}$ passando pela origem tais que:

(i) acima da curva $D_{23}, \mathbf{S}_{\tau_{0}, \varepsilon, \mu}$ não possui pontos umbílicos em uma vizinhança do ponto $\delta\left(u_{0}\right)$ em $\mathbf{S}_{\tau_{0}, \varepsilon, \mu} ;$

(ii) entre as curvas $D_{23}$ e $D_{12}, \mathbf{S}_{\tau_{0}, \varepsilon, \mu}$ possui dois pontos umbilicos Darbouxianos dos tipos $D_{2}$ e $D_{3}$ em uma vizinhança do ponto $\delta\left(u_{0}\right)$ em $\mathbf{S}_{\tau_{0}, \varepsilon, \mu}$;

(iii) abaixo da curva $D_{12}, \mathbf{S}_{\tau_{0}, \varepsilon, \mu}$ possui dois pontos umbilicos Darbouxianos dos tipos $D_{1}$ e $D_{3}$ em uma vizinhança do ponto $\delta\left(u_{0}\right)$ em $\mathbf{S}_{\tau_{0}, \varepsilon, \mu}$.

(iv) para cada ponto da curva $D_{23}$ existe um único ponto umbílico não Darbouxiano do tipo $D_{23}$ em uma vizinhança do ponto $\delta\left(u_{0}\right)$ em $\mathbf{S}_{\tau_{0}, \varepsilon, \mu}$;

(v) para cada ponto da curva $D_{12}$ existe um único ponto umbílico não Darbouxiano do tipo $D_{12}$ em uma vizinhança do ponto $\delta\left(u_{0}\right)$ em $\mathbf{S}_{\tau_{0}, \varepsilon, \mu}$.

Estas situações estão ilustradas no plano $\Pi$ da figura 4.8 .

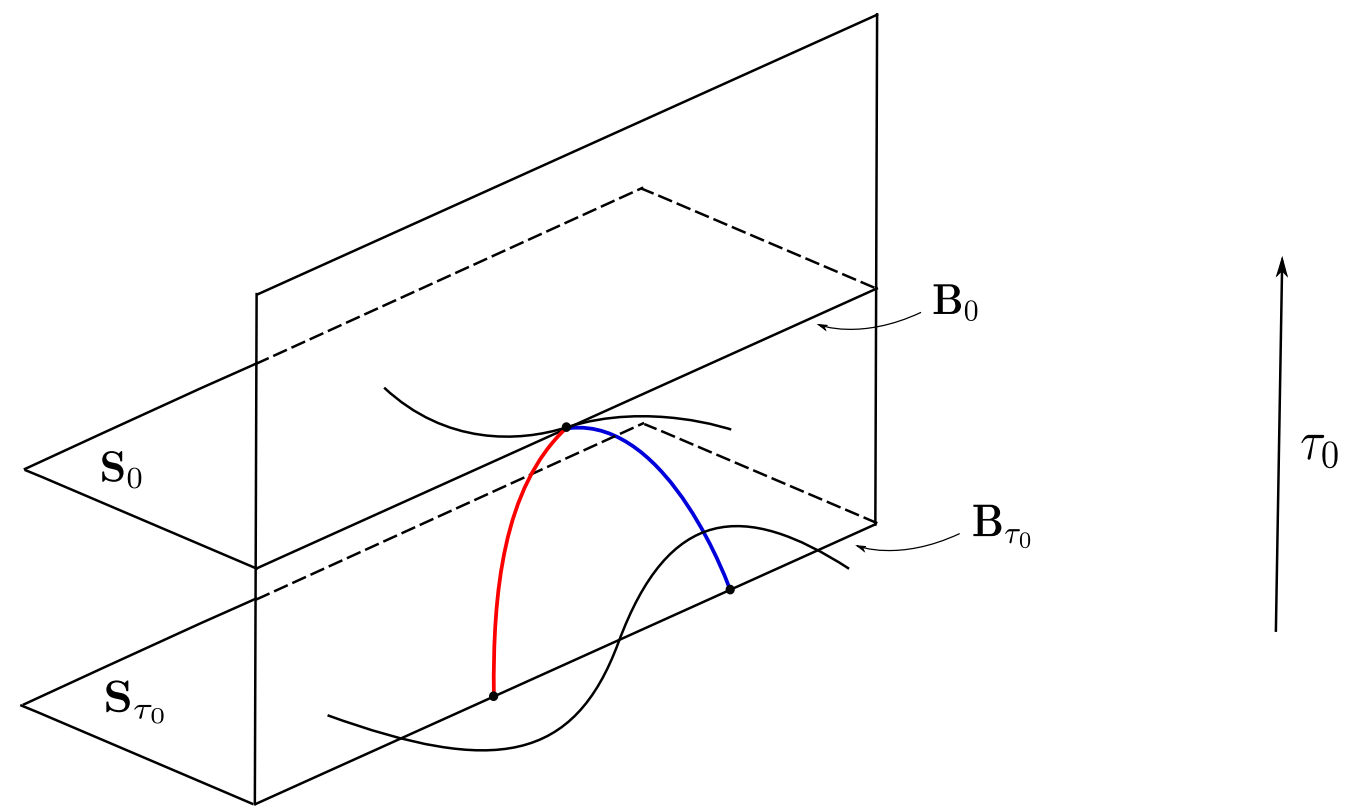

Figura 4.7: Familia a 1 parâmetro de superfícies $\mathbf{S}_{\tau_{0}}$ com bordo variável $\mathbf{B}_{\tau_{0}}$. Se $\tau_{0}=0$ temos um ponto de contato cúbico entre as linhas de curvatura principal mínima de $\mathbf{S}_{0}=\mathbf{S}$ com o bordo $\mathbf{B}_{0}=\mathbf{B}$. Com relação à superfície $\mathbf{S}_{\tau_{0}}\left(\tau_{0}<0\right)$, o contato das linhas de curvatura de $\mathbf{S}_{\tau_{0}}$ com $\mathbf{B}_{\tau_{0}}$ é quadrático externo (curva azul) e quadrático interno (curva vermelha). Destes pontos de tangências quadráticas externa e interna emergem pontos umbílicos Darbouxianos, respectivamente, dos tipos $D_{1}$ e $D_{3}$; isto foi demonstrado por Garcia e Sotomayor em [5], em um contexto local específico e para valores pequenos do parâmetro $\varepsilon>0$.

Demonstração. Vamos dividir a demonstração em duas partes: na primeira delas determinaremos os pontos umbílicos não Darbouxianos do tipo $D_{23}$ e, na segunda, os pontos umbílicos não Darbouxianos do tipo $D_{12}$. 


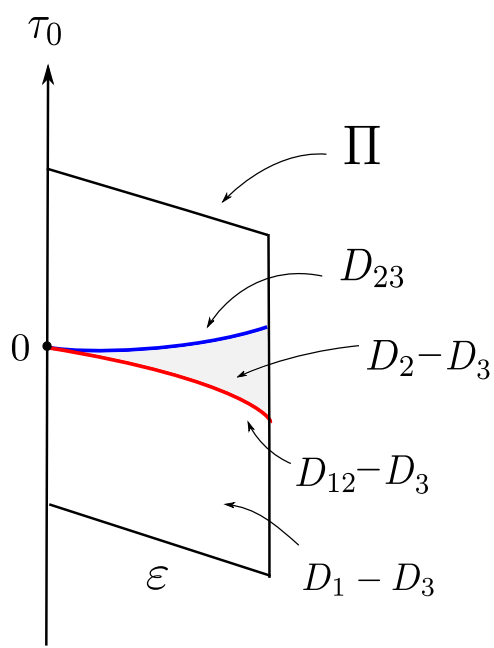

Figura 4.8: Diagrama de bifurcação em uma vizinhança de um ponto de contato cúbico.

\section{Pontos umbílicos não Darbouxianos do tipo $D_{23}$}

Vimos na seção 1.3 .2 do capítulo 1 que o ponto umbílico não Darbouxiano $D_{23}$ ocorre quando a condição de regularidade, $T$, da definição 1.2 .2 é desrespeitada e a condição do discriminante, $D$, é preservada. Veja definição do ponto $D_{23}$ em 1.3 .4

Vamos supor, sem perda de generalidade, que $u_{0}=0$.

Considere na expressão de $N(u, w, \varepsilon)$ em (4.24) que a torção geodésica $\tau_{g}(u)$ tem a seguinte expansão de Taylor na variável $u$, até ordem 3 , na vizinhança de $u=0$.

$$
\tau_{g}(u)=\tau_{0}+\frac{\tau_{g}^{\prime \prime}(0)}{2} u^{2}+\frac{\tau_{g}^{\prime \prime \prime}(0)}{6} u^{3}+O(4),
$$

onde $\tau_{g}^{\prime}(0)=0, \tau_{g}^{\prime \prime}(0) \neq 0$, por hipótese do teorema e $\tau_{0}:=\tau_{g}(0)$.

Iremos seguir os seguinte passos:

(1) Nosso primeiro objetivo é determinar os pontos umbílicos não Darbouxianos $D_{23}$. Três condições necessárias para termos pontos umbílicos $D_{23}$ são dadas pelas equações do seguinte sistema:

$$
\left\{\begin{array}{rl}
M(u, w, \varepsilon) & =0 \\
N\left(\tau_{0}, u, w, \varepsilon\right) & =0 \\
\operatorname{det}\left(\frac{\partial(M, N)}{\partial(u, w)}\right) & =0
\end{array},\right.
$$

sendo as expressões de $M$ e $N$ obtidas em (4.24).

Vamos resolver $M(u, w, \varepsilon)=0$. Como $\varepsilon>0$, observe que $M(u, w, \varepsilon)=0$ se, e somente 
se,

$$
\begin{aligned}
\tilde{M}(u, w, \varepsilon) & =\left[-1+\phi(w)+2 w \phi^{\prime}(w)+\frac{1}{2} w^{2} \phi^{\prime \prime}(w)\right]+ \\
& +\varepsilon\left[k_{n}^{\perp}(u)-k_{n}(u)+k_{n}^{\perp}(u)\left(-1+\phi(w)+2 w \phi^{\prime}(w)+\frac{1}{2} w^{2} \phi^{\prime \prime}(w)\right)\right]+ \\
& +\varepsilon^{2}(\cdots)=0,
\end{aligned}
$$

onde $\widetilde{M}(u, w, \varepsilon)=\frac{M(u, w, \varepsilon)}{\varepsilon^{5}}$.

Logo, das propriedades da função de transição $\phi$, temos:

$$
\begin{gathered}
\tilde{M}(0,0,0)=-1+\phi(0)=0 \text { e } \\
\tilde{M}_{\varepsilon}(0,0,0)=\phi(0) k_{n}^{\perp}(0)-k_{n}(0)=k_{n}^{\perp}(0)-k_{n}(0) \neq 0,
\end{gathered}
$$

por hipótese do teorema. Resolvendo $\tilde{M}(u, w, \varepsilon)=0$, em uma vizinhança da origem, pelo Teorema da Função Implícita, existe uma função suave $\varepsilon(u, w)$ tal que $\tilde{M}(u, w, \varepsilon(u, w))=$ 0 e $\varepsilon(0,0)=0$.

Após algumas diferenciações, obtemos a seguinte expansão de Taylor nas variáveis $u$ e $w$, até a ordem 2, para a função $\varepsilon(u, w)$ em uma vizinhança de $u=w=0$.

$$
\begin{aligned}
\varepsilon(u, w) & =\frac{1-\phi(0)}{\left(\phi k_{n}^{\perp}-k_{n}\right)(0)}+\frac{(-1+\phi(0))\left[\phi\left(k_{n}^{\perp}\right)^{\prime}-k_{n}^{\prime}\right](0)}{\left[\left(\phi k_{n}^{\perp}-k_{n}\right)(0)\right]^{2}} u+\frac{2 \phi^{\prime}(0)\left(k_{n}-k_{n}^{\perp}\right)(0)}{\left[\left(\phi k_{n}^{\perp}-k_{n}\right)(0)\right]^{2}} w- \\
& -(1-\phi(0))\left\{\frac{\left[\phi\left(k_{n}^{\perp}\right)^{\prime \prime}-k_{n}^{\prime \prime}\right](0)}{2\left[\left(\phi k_{n}^{\perp}-k_{n}\right)(0)\right]^{2}}-\frac{\left[k_{n}^{\prime}(0)-\phi(0)\left(k_{n}^{\perp}\right)^{\prime}(0)\right]^{2}}{6\left[\left(\phi k_{n}^{\perp}-k_{n}\right)(0)\right]^{3}}\right\} u^{2}+ \\
& +2\left\{\frac{(1-\phi(0)) \phi^{\prime}(0)\left(k_{n}^{\perp}\right)^{\prime}(0)}{\left[\left(\phi k_{n}^{\perp}-k_{n}\right)(0)\right]^{2}}-\frac{\left[\phi^{\prime}\left(k_{n}-k_{n}^{\perp}\right)\right]\left[\phi\left(k_{n}^{\perp}\right)^{\prime}-k_{n}^{\prime}\right](0)}{\left[\left(\phi k_{n}^{\perp}-k_{n}\right)(0)\right]^{3}}-\right. \\
& \left.-\frac{\left(\phi^{\prime} k_{n}\right)(0)\left[(1-\phi)\left(k_{n}^{\prime}-\phi\left(k_{n}^{\perp}\right)^{\prime}\right)\right](0)}{\left[\left(\phi k_{n}^{\perp}-k_{n}\right)(0)\right]^{3}}\right\} u w+ \\
& +\frac{1}{2}\left\{\frac{\left(k_{n}-k_{n}^{\perp}\right)(0)\left[\phi^{\prime \prime}\left(\phi k_{n}^{\perp}-k_{n}\right)-8\left(\phi^{\prime}\right)^{2} k_{n}^{\perp}\right](0)}{\left[\left(\phi k_{n}^{\perp}-k_{n}\right)(0)\right]^{3}}\right\} w^{2}+O(3) .
\end{aligned}
$$

Agora, vamos resolver $N\left(\tau_{0}, u, w, \varepsilon\right)=0$. Como $\varepsilon>0$, observe da expressão de $N$ em 
4.24 que $N\left(\tau_{0}, u, w, \varepsilon\right)=0$ se, e somente se, $\tilde{N}\left(\tau_{0}, u, w, \varepsilon\right)=0$, sendo $\tilde{N}=\frac{N}{\varepsilon^{4}}$.

Substituindo $\varepsilon=\varepsilon(u, w)$ da equação 4.28 em $\tilde{N}\left(\tau_{0}, u, w, \varepsilon\right)$, obtemos

$$
\tilde{N}\left(\tau_{0}, u, w\right)=\tilde{N}\left(\tau_{0}, u, w, \varepsilon(u, w)\right) .
$$

A equação $\tilde{N}\left(\tau_{0}, u, w\right)=0$ é a equação a ser resolvida, em uma vizinhança do ponto $\left(\tau_{0}, u, w\right)=(0,0,0)$.

Das propriedades da função de transição $\phi$, temos que $\tilde{N}(0,0,0)=0$ e

$$
\begin{aligned}
\frac{\partial \tilde{N}}{\partial \tau_{0}}(0,0,0) & =864\left[\left(\phi k_{n}^{\perp}-k_{n}\right)(0)\right]^{4}\left\{6[\phi(0)-1]^{3} \tau_{g}^{2}(0)+\left[\left(\phi k_{n}^{\perp}-k_{n}\right)(0)\right]^{2}\right\}= \\
& =864\left[\left(k_{n}^{\perp}-k_{n}\right)(0)\right]^{6} \neq 0,
\end{aligned}
$$

por hipótese do teorema. Pelo Teorema da Função Implícita, existe uma função suave $\tau_{0}=\tau_{0}(u, w)$ tal que $\tilde{N}\left(\tau_{0}(u, w), u, w\right)=0$ e $\tau_{0}(0,0)=0$.

Assim, obtemos a seguinte expansão de Taylor nas variáveis $u$ e $w$, até a ordem 2 , da função $\tau_{0}(u, w)$ em uma vizinhança de $(u, w)=(0,0)$ :

$$
\begin{aligned}
\tau_{0}(u, w) & =-\frac{\left[\phi(\phi-1)^{2}\left(k_{n}^{\perp}\right)^{\prime}\right](0)}{\left[\left(\phi k_{n}^{\perp}-k_{n}\right)(0)\right]^{2}} w-\frac{\tau_{g}^{\prime \prime}(0)}{2} u^{2}- \\
& -\phi(0)[(\phi-1)(0)]^{2}\left\{\frac{\left(k_{n}^{\perp}\right)^{\prime \prime}(0)}{\left[\left(\phi k_{n}^{\perp}-k_{n}\right)(0)\right]^{2}}+\frac{2\left(k_{n}^{\perp}\right)^{\prime}(0)\left[k_{n}^{\prime}-\phi\left(k_{n}^{\perp}\right)^{\prime}\right](0)}{\left[\left(\phi k_{n}^{\perp}-k_{n}\right)(0)\right]^{3}}\right\} u w+ \\
& +\frac{\left[(\phi-1)\left(k_{n}^{\perp}\right)^{\prime}\right](0)}{2}\left\{\frac{\phi^{\prime}(0)\left[\phi\left(9 k_{n}-7 k_{n}^{\perp}-\phi k_{n}^{\perp}\right)-k_{n}\right](0)}{\left[\left(\phi k_{n}^{\perp}-k_{n}\right)(0)\right]^{3}}+\right. \\
& \left.+\frac{4\left[\phi(\phi-1)^{3} k_{g}\right](0)}{\left[\left(\phi k_{n}^{\perp}-k_{n}\right)(0)\right]^{4}}\right\} w^{2}+O(3) .
\end{aligned}
$$

Por fim resolveremos a terceira equação do sistema (4.27), dada por:

$$
d:=\operatorname{det}\left(\frac{\partial(M, N)}{\partial(u, w)}\right)=0
$$

Usando as expressões de $M$ e $N$ da equação (4.24) e considerando, como antes, que

$$
\tau_{g}(u)=\tau_{0}+\frac{\tau_{g}^{\prime \prime}(0)}{2} u^{2}+\frac{\tau_{g}^{\prime \prime \prime}(0)}{6} u^{3}+O(4),
$$


onde $\tau_{g}^{\prime}(0)=0, \tau_{g}^{\prime \prime}(0) \neq 0$, por hipótese do teorema e $\tau_{0}:=\tau_{g}(0)$, obtemos, após alguns cálculos, o seguinte:

$$
\begin{aligned}
d=d\left(\tau_{0}, u, w, \varepsilon\right) & =-3 \tau_{g}^{\prime \prime}(0)\left[w \phi^{\prime \prime}(w)+\phi^{\prime}(w)\right] u-3 \tau_{g}^{\prime \prime \prime}(0)\left[w \phi^{\prime \prime}(w)+\phi^{\prime}(w)\right] u^{2}- \\
& -\frac{3}{2} w\left[w \phi^{\prime \prime}(w)+\phi^{\prime}(w)\right]\left\{\left(k_{n}^{\perp}\right)^{\prime \prime}(0)\left[2 \phi(w)+w \phi^{\prime}(w)\right]-4 \tau_{0} k_{g}^{\prime}(0)\right\} \varepsilon^{2}- \\
& -3 \tau_{g}^{\prime \prime}(0) k_{n}^{\perp}(0)\left[w \phi^{\prime \prime}(w)+\phi^{\prime}(w)\right] u \varepsilon+O(3) .
\end{aligned}
$$

O próximo passo será substituir neste determinante jacobiano, $d\left(\tau_{0}, u, w, \varepsilon\right)$, as expressões obtidas para $\varepsilon=\varepsilon(u, w)$ em 4.28 e $\tau_{0}=\tau_{0}(u, w)$ em 4.29). Fazendo isso, teremos a seguinte equação a ser resolvida em uma vizinhança de $(u, w)=(0,0)$.

$$
d(u, w)=d\left(\tau_{0}(u, w), u, w, \varepsilon(u, w)\right)=0 .
$$

A solução de 4.30 tem a seguinte expressão: ${ }^{2}$

$$
\begin{aligned}
u_{23} & =\left\{\frac{-[\phi(0)-1]^{2}\left(k_{n}^{\perp}\right)^{\prime \prime}(0)\left[\phi(0) k_{n}(0)-k_{n}^{\perp}(0)\right]}{\tau_{g}^{\prime \prime}(0)\left[k_{n}(0)-k_{n}^{\perp}(0)\right]^{3}}\right\} w+ \\
& +\left\{\frac{6 \phi^{\prime}(0)\left(k_{n}^{\perp}\right)^{\prime \prime}(0)[1-\phi(0)]}{\tau_{g}^{\prime \prime}(0)\left[k_{n}(0)-k_{n}^{\perp}(0)\right]^{2}}\right\} w^{2}+O(3) .
\end{aligned}
$$

Substituindo $u_{23}=u_{23}(w)$ da equação 4.31 nas expressões para $\varepsilon=\varepsilon(u, w)$ em 4.28 e para $\tau_{0}=\tau_{0}(u, w)$ em 4.29 , obtemos a seguinte equação paramétrica, com parâmetro $w$, para os pontos umbílicos do tipo $D_{23}$ :

$$
\left\{\begin{array}{rl}
\varepsilon_{23}(w) & =\varepsilon_{23}\left(u_{23}(w), w\right) \\
\left(\tau_{0}\right)_{23}(w) & =\left(\tau_{0}\right)_{23}\left(u_{23}(w), w\right)
\end{array},\right.
$$

\footnotetext{
${ }^{2} \mathrm{~A}$ notação $u_{23}$ na equação 4.31 faz referência ao caso que estamos demonstrando: determinar os pontos umbílicos do tipo $D_{23}$.
} 
sendo

$$
\begin{aligned}
\varepsilon_{23}(w)= & \frac{[\phi-1]\left[k_{n}^{\perp}(0)(\phi-2)+k_{n}(0)\right]}{\left[k_{n}(0)-k_{n}^{\perp}(0)\right]^{2}}+ \\
+ & \frac{3 \phi^{\prime}}{\left[k_{n}(0)-k_{n}^{\perp}(0)\right]^{3}}\left\{\left(k_{n}^{\perp}(0)\right)^{2}\left[3 \phi^{2}-8 \phi+6\right]+2 k_{n}(0) k_{n}^{\perp}(0)(\phi-2)+k_{n}^{2}(0)\right\} w+ \\
+ & \left\{\frac{9 \phi^{\prime}\left(k_{n}^{\perp}\right)^{\prime \prime}(0)(\phi-1)^{2}}{\tau_{g}^{\prime \prime}(0)\left[k_{n}(0)-k_{n}^{\perp}(0)\right]^{3}}+\frac{1}{2\left[k_{n}(0)-k_{n}^{\perp}(0)\right]^{3}}\left[39 k_{g}(0) \phi^{\prime}\left(2 \phi-\phi^{2}-1\right)+\right.\right. \\
+ & \left.6 k_{n}^{\perp}(0)\left(3 \phi k_{n}^{\perp}(0)+2 k_{n}(0)-8 k_{n}^{\perp}(0)\right) \phi \phi^{\prime \prime}+6\left(k_{n}^{2}-4 k_{n}^{\perp} k_{n}+6\left(k_{n}^{\perp}\right)^{2}\right)(0) \phi^{\prime \prime}\right]+ \\
+ & \frac{18\left(\phi^{\prime}\right)^{2}}{2\left[k_{n}(0)-k_{n}^{\perp}(0)\right]^{4}}\left[6\left(k_{n}^{\perp}(0)\right)^{3} \phi^{2}+3\left(k_{n}^{\perp}(0)\right)^{2}\left(k_{n}(0)-5 k_{n}^{\perp}(0)\right) \phi+\right. \\
+ & \left.\left.\left.k_{n}^{\perp}(0)\left(k_{n}^{2}-5 k_{n} k_{n}^{\perp}+10\left(k_{n}^{\perp}\right)^{2}\right)(0)\right]\right\} w^{2}+\cdots, 33\right) \\
+ & \frac{\left(k_{n}^{\perp}\right)^{\prime}(0) \phi^{\prime}(\phi-1)}{2\left[k_{n}(0)-k_{n}^{\perp}(0)\right]^{4}}\left\{\left(k_{n}^{\perp}(0)\right)^{2}\left[34 \phi^{2}-91 \phi+69\right]+\right. \\
\left(\tau_{0}\right)_{23}(w)=\left\{\frac{\left(k_{n}^{\perp}\right)^{\prime}(0)(\phi-1)^{2}\left[2 k_{n}^{\perp}(0)-\phi k_{n}^{\perp}(0)-\phi k_{n}(0)\right]}{\left[k_{n}(0)-k_{n}^{\perp}(0)\right]^{3}}\right\} & \left.(0)\left[19 \phi^{2}-33 \phi+2\right]+k_{n}^{2}(0)(13 \phi-1)\right\} w^{2}+
\end{aligned}
$$

(2) Para que os pontos descritos pelas equações em 4.32 sejam, de fato, do tipo $D_{23}$, falta verificar que, ao longo destes pontos, o contato entre $M(u, w, \varepsilon)=0$ e $N(u, w, \varepsilon)=0$ é quadrático. A seguir, vamos verificar esta condição no espaço de parâmetros $\varepsilon$ e $\tau_{0}$.

Seja $J$ a seguinte matriz jacobiana:

$$
J:=\frac{\partial\left(\varepsilon, \tau_{0}\right)}{\partial(u, w)}=\left[\begin{array}{cc}
\frac{\partial \varepsilon}{\partial u}(u, w) & \frac{\partial \varepsilon}{\partial w}(u, w) \\
\frac{\partial \tau_{0}}{\partial u}(u, w) & \frac{\partial \tau_{0}}{\partial w}(u, w)
\end{array}\right]
$$

onde as expressões de $\varepsilon=\varepsilon(u, w)$ e $\tau_{0}=\tau_{0}(u, w)$ são as dadas, respectivamente, pelas 
equações 4.28) e 4.29).

Considere $\operatorname{adj}(J)$, a matriz adjunta de $J$, logo

$$
\operatorname{adj}(J)=\left[\begin{array}{cc}
a_{11}(u, w) & a_{12}(u, w) \\
a_{21}(u, w) & a_{22}(u, w)
\end{array}\right]=\left[\begin{array}{cc}
\frac{\partial \tau_{0}}{\partial w}(u, w) & -\frac{\partial \tau_{0}}{\partial u}(u, w) \\
-\frac{\partial \varepsilon}{\partial w}(u, w) & \frac{\partial \varepsilon}{\partial u}(u, w)
\end{array}\right] .
$$

Denote por $b_{11}=b_{11}(w), b_{21}=b_{21}(w)$ as respectivas restrições de $a_{11}(u, w)$ e $a_{21}(u, v)$ (poderíamos, também, ter escolhido a segunda coluna da matriz $\operatorname{adj}(J)$ ) à curva $u_{23}=$ $u_{23}(w)$ dada pela equação 4.31.

Afirmamos que o determinante da matriz $W$ é não nulo, sendo

$$
W:=\left[\begin{array}{cc}
b_{11}(w) & \frac{d}{d u} u_{23}(w) \\
b_{21}(w) & \frac{d}{d w} u_{23}(w)
\end{array}\right]=\left[\begin{array}{cc}
b_{11}(w) & 1 \\
b_{21}(w) & \frac{d}{d w} u_{23}(w)
\end{array}\right]
$$

De fato,

$$
\begin{aligned}
\operatorname{det}(W) & =b_{11}(w) \cdot \frac{d}{d w} u_{23}(w)-b_{21}(w)=\frac{(\phi(w)-1)}{\left(\tau_{g}^{\prime \prime}(0)\right)^{2}\left[k_{n}(0)-k_{n}^{\perp}(0)\right]^{6}}\{w(\cdots)+ \\
& +\phi(w)\left[k_{n}^{\perp}\left(k_{n}^{\perp}\right)^{\prime}\left(k_{n}^{\perp}\right)^{\prime \prime} \tau_{g}^{\prime \prime}\right](0)\left[k_{n}(0)-k_{n}^{\perp}(0)\right]^{3}\left(k_{n}(0)\right)^{2}- \\
& -\phi^{2}(w)\left[\left(k_{n}^{\perp}\right)^{\prime}\left(k_{n}^{\perp}\right)^{\prime \prime} k_{n} \tau_{g}^{\prime \prime}\right](0)\left[k_{n}(0)-k_{n}^{\perp}(0)\right]^{3}\left[k_{n}(0)+k_{n}^{\perp}(0)\right] . \\
& \left.\cdot\left[k_{n}(0)+2 k_{n}^{\perp}(0)\right]+\phi^{3}(w)(\ldots)\right\},
\end{aligned}
$$

onde observamos que o denominador $\tau_{g}^{\prime \prime}(0)\left[k_{n}(0)-k_{n}^{\perp}(0)\right]$ é não nulo, por hipótese.

Segue das propriedades da função de transição $\phi$ que, para $w$ próximo de $0, \phi(w)$ está próximo de 1, porém é sempre menor que 1, uma vez que $\phi$ é uma função decrescente. Portanto, para $w$ próximo de $0, \operatorname{det}(W)$ em (4.35) é não nulo se, e somente se, o coeficiente de $\phi(w)$ dado por

$$
\left[k_{n}^{\perp}\left(k_{n}^{\perp}\right)^{\prime}\left(k_{n}^{\perp}\right)^{\prime \prime} \tau_{g}^{\prime \prime}\right](0)\left[k_{n}(0)-k_{n}^{\perp}(0)\right]^{3}\left(k_{n}(0)\right)^{2}
$$

é não nulo.

Como, por hipótese do teorema, $k_{n}^{\perp}(0),\left(k_{n}^{\perp}\right)^{\prime}(0),\left(k_{n}^{\perp}\right)^{\prime \prime}(0)$ e $k_{n}(0)$ são todos não nulos, temos $\operatorname{det}(W) \neq 0$ para $w$ próximo de 0 .

Portanto, 4.32 é a imagem de uma dobra de Whitney e, para cada $w,\left(u_{23}(w), w\right)$ 
são as coordenadas do ponto umbílico não Darbouxiano $D_{23}$. Ver [14] para mais detalhes sobre a Teoria de Whitney.

\section{Pontos umbílicos não Darbouxianos do tipo $D_{12}$}

Na segunda parte da demonstração vamos determinar os pontos umbílicos do tipo $D_{12}$.

Como antes suponha, sem perda de generalidade, que $u_{0}=0$.

Vimos na seção 1.3.1 do capítulo 1 que o ponto umbílico não Darbouxiano $D_{12}$ ocorre quando a condição do discriminante, $D$, da definição 1.2 .2 é desrespeitada e a condição de transversalidade, $T$, é preservada. Veja definição do ponto $D_{12}$ em 1.3 .1 .

Também vimos no capítulo1, definição 1.2.1, que na carta $(u, w ; p)$, sendo $p=d w / d u$, consideramos a superfície de Lie-Cartan $\mathcal{L}^{-1}\{0\}$ sobre a qual temos definido o campo de Lie-Cartan $X$, sendo:

$$
\mathcal{L}(u, w, \varepsilon ; p)=L(u, w, \varepsilon) p^{2}+M(u, w, \varepsilon) p+N(u, w, \varepsilon)=0,
$$

$L, M, N$ dadas pelas expressões da equação 4.24 e

$$
X=\mathcal{L}_{p} \frac{\partial}{\partial u}+p \mathcal{L}_{p} \frac{\partial}{\partial w}-\left(\mathcal{L}_{u}+p \mathcal{L}_{w}\right) \frac{\partial}{\partial p} .
$$

Lembremos que as singularidades de $X$ são $(0,0 ; p)$, sendo $p$ as raízes do polinômio cúbico $\Psi(p)=-\left(\mathcal{L}_{u}+p \mathcal{L}_{w}\right)$.

A condição $D_{12}$ é equivalente à existência de uma raiz dupla e não nula do polinômio cúbico $\Psi(p)=-\left(\mathcal{L}_{u}+p \mathcal{L}_{w}\right)$.

Vamos denotar por result $\left(\Psi(p), \Psi^{\prime}(p), p\right)$ o resultante dos polinômios $\Psi(p)$ e $\Psi^{\prime}(p)$. Portanto, $\Psi(p)$ possui uma raiz dupla se, e somente se, result $\left(\Psi(p), \Psi^{\prime}(p), p\right)=0 .{ }^{3}$

(3) Nosso objetivo é determinar os pontos umbílicos não Darbouxianos $D_{12}$. Três condições necessárias para termos pontos umbílicos do tipo $D_{12}$ são dadas pelas equações do seguinte sistema:

$$
\left\{\begin{aligned}
M(u, w, \varepsilon) & =0 \\
N\left(\tau_{0}, u, w, \varepsilon\right) & =0 \\
\operatorname{result}\left(\Psi(p), \Psi^{\prime}(p), p\right) & =0
\end{aligned}\right.
$$

sendo as expressões de $M(u, w, \varepsilon)$ e $N(u, w, \varepsilon)$ dadas na equação 4.24 e lembrando que na expressão para $N(u, w, \varepsilon)$ a torção geodésica $\tau_{g}(u)$ tem a seguinte expansão de Taylor na variável $u$, até ordem 3 , na vizinhança de $u=0$ :

$$
\tau_{g}(u)=\tau_{0}+\frac{\tau_{g}^{\prime \prime}(0)}{2} u^{2}+\frac{\tau_{g}^{\prime \prime \prime}(0)}{6} u^{3}+O(4)
$$

\footnotetext{
${ }^{3} \mathrm{O}$ resultante de dois polinômios é o determinante da matriz de Sylvester associada a estes polinômios.
} 
onde $\tau_{g}^{\prime}(0)=0, \tau_{g}^{\prime \prime}(0) \neq 0$, por hipótese do teorema e $\tau_{0}:=\tau_{g}(0)$.

Após alguns cálculos obtemos:

$$
\begin{aligned}
\operatorname{result}\left(\Psi(p), \Psi^{\prime}(p), p\right) & =L_{w}\left\{-2 M_{w} N_{w}^{2} L_{u}-18 L_{w} M_{w} N_{w} N_{u}-2 M_{w}^{2} N_{w} M_{u}-\right. \\
& -18 L_{w} L_{u} N_{w} N_{u}-2 L_{u}^{2} N_{w} M_{u}-18 L_{w} N_{u} M_{u} L_{u}-18 L_{w} N_{u} M_{u} M_{w}- \\
& -2 L_{u} M_{u}^{2} M_{w}+12 L_{u} M_{w}^{2} N_{u}+12 M_{w} L_{u}^{2} N_{u}+27 L_{w}^{2} N_{u}^{2}+4 L_{w} N_{w}^{3}+ \\
& +4 L_{w} M_{u}^{3}+12 L_{w} N_{w} M_{u}^{2}-M_{w}^{2} N_{w}^{2}-L_{u}^{2} N_{w}^{2}-L_{u}^{2} M_{u}^{2}-M_{w}^{2} M_{u}^{2}- \\
& \left.+4 M_{w}^{3} N_{u}+4 L_{u}^{3} N_{u}+12 L_{w} N_{w}^{2} M_{u}-4 M_{w} N_{w} M_{u} L_{u}\right\},
\end{aligned}
$$

onde $\frac{\partial L}{\partial u}=L_{u}, \frac{\partial L}{\partial w}=L_{w}, \frac{\partial M}{\partial u}=M_{u}, \quad \frac{\partial M}{\partial w}=M_{w}, \quad \frac{\partial N}{\partial u}=N_{u}$ e $\frac{\partial N}{\partial w}=N_{w}$.

Seja

$$
R\left(\tau_{0}, u, w, \varepsilon\right):=\frac{\operatorname{result}\left(\Psi(p), \Psi^{\prime}(p), p\right)}{L_{w}} .
$$

Substituindo neste resultante $R\left(\tau_{0}, u, w, \varepsilon\right)$, as expressões obtidas para $\varepsilon=\varepsilon(u, w)$ em (4.28) e $\tau_{0}=\tau_{0}(u, w)$ em 4.29), teremos a seguinte equação a ser resolvida em uma vizinhança de $(u, w)=(0,0)$ :

$$
R(u, w)=R\left(\tau_{0}(u, w), u, w, \varepsilon(u, w)\right)=0 .
$$

A curva solução de 4.39 tem a seguinte expressão:

$$
\begin{aligned}
u & =\frac{[\phi(w)-1]^{3}\left[k_{n}^{\prime}(0)-2\left(k_{n}^{\perp}\right)^{\prime}(0)\right]^{2}}{12 \phi^{\prime}(w) \tau_{g}^{\prime \prime}(0)\left[k_{n}(0)-k_{n}^{\perp}(0)\right]^{3}}-\left\{\frac{[\phi(w)-1]^{2}\left(k_{n}^{\perp}\right)^{\prime \prime}(0)}{\tau_{g}^{\prime \prime}(0)\left[k_{n}(0)-k_{n}^{\perp}(0)\right]^{2}}-\right. \\
& \left.-\frac{3[\phi(w)-1]^{2}\left[k_{n}^{\prime}(0)-2\left(k_{n}^{\perp}\right)^{\prime}(0)\right]^{2}}{2 \tau_{g}^{\prime \prime}(0)\left[k_{n}(0)-k_{n}^{\perp}(0)\right]^{3}}\right\} w+\cdots .
\end{aligned}
$$

Vale observar que a primeira parcela da equação 4.40 dada por:

$$
\frac{[\phi(w)-1]^{3}\left[k_{n}^{\prime}(0)-2\left(k_{n}^{\perp}\right)^{\prime}(0)\right]^{2}}{12 \phi^{\prime}(w) \tau_{g}^{\prime \prime}(0)\left[k_{n}(0)-k_{n}^{\perp}(0)\right]^{3}}
$$

é uma indeterminação pois para $w$ próximo de $0, \phi(w)$ está próximo de 1 e $\phi^{\prime}(w)$ próximo de 0 , das propriedades da função de transição $\phi$. No entanto, pelo Teorema do 
Valor Médio, podemos considerar $\phi(w)=1+w \phi^{\prime}\left(w^{*}\right), w^{*} \in(0, w)$. Como $w$ está suficientemente próximo de 0 , continuaremos usando $w$ ao invés de $w^{*}$. Portanto, sendo $\phi(w)=1+w \phi^{\prime}(w)$, para $w$ próximo de 0 , concluímos que $\frac{[\phi(w)-1]^{3}}{\phi^{\prime}(w)}$ está próximo de $w^{3}\left[\phi^{\prime}(w)\right]^{2}$. Por fim, considerando $\phi(w)=1+w \phi^{\prime}(w)$ na equação 4.40 obtemos:

$$
u_{12}=\left\{\frac{19\left[\phi^{\prime}(w)\right]^{2}\left[k_{n}^{\prime}(0)-2\left(k_{n}^{\perp}\right)^{\prime}(0)\right]^{2}}{12 \tau_{g}^{\prime \prime}(0)\left[k_{n}(0)-k_{n}^{\perp}(0)\right]^{3}}-\frac{\left(k_{n}^{\perp}\right)^{\prime \prime}(0)\left[\phi^{\prime}(w)\right]^{2}}{\tau_{g}^{\prime \prime}(0)\left[k_{n}(0)-k_{n}^{\perp}(0)\right]^{2}}\right\} w^{3}+\cdots
$$

Substituindo $u_{12}=u_{12}(w)$ da equação 4.41 nas expressões para $\varepsilon=\varepsilon(u, w)$ em 4.28 e para $\tau_{0}=\tau_{0}(u, w)$ em 4.29 , obtemos a seguinte equação paramétrica, com parâmetro $w$, para a curva $D_{12}$ :

$$
\left\{\begin{array}{rl}
\varepsilon_{12}(w) & =\varepsilon_{12}\left(u_{12}(w), w\right) \\
\left(\tau_{0}\right)_{12}(w) & =\left(\tau_{0}\right)_{12}\left(u_{12}(w), w\right)
\end{array},\right.
$$

sendo

$$
\begin{aligned}
\varepsilon_{12}(w) & =\frac{[\phi-1]\left[k_{n}^{\perp}(0)(\phi-2)+k_{n}(0)\right]}{\left[k_{n}(0)-k_{n}^{\perp}(0)\right]^{2}}+ \\
& +\frac{3 \phi^{\prime}}{\left[k_{n}(0)-k_{n}^{\perp}(0)\right]^{3}}\left\{\left(k_{n}^{\perp}(0)\right)^{2}\left[3 \phi^{2}-8 \phi+6\right]+2 k_{n}(0) k_{n}^{\perp}(0)(\phi-2)+k_{n}^{2}(0)\right\} w+ \\
& +\left\{\frac { 1 } { 2 [ k _ { n } ( 0 ) - k _ { n } ^ { \perp } ( 0 ) ] ^ { 3 } } \left[39 k_{g}(0) \phi^{\prime}\left(2 \phi-\phi^{2}-1\right)+\right.\right. \\
& \left.+6 k_{n}^{\perp}(0)\left(3 \phi k_{n}^{\perp}(0)+2 k_{n}(0)-8 k_{n}^{\perp}(0)\right) \phi \phi^{\prime \prime}+6\left(k_{n}^{2}-4 k_{n}^{\perp} k_{n}+6\left(k_{n}^{\perp}\right)^{2}\right)(0) \phi^{\prime \prime}\right]+ \\
& +\frac{18\left(\phi^{\prime}\right)^{2}}{2\left[k_{n}(0)-k_{n}^{\perp}(0)\right]^{4}}\left[6\left(k_{n}^{\perp}(0)\right)^{3} \phi^{2}+3\left(k_{n}^{\perp}(0)\right)^{2}\left(k_{n}(0)-5 k_{n}^{\perp}(0)\right) \phi+\right. \\
& \left.\left.+k_{n}^{\perp}(0)\left(k_{n}^{2}-5 k_{n} k_{n}^{\perp}+10\left(k_{n}^{\perp}\right)^{2}\right)(0)\right]\right\} w^{2}+\cdots,
\end{aligned}
$$

\footnotetext{
${ }^{4} \mathrm{~A}$ notação $u_{12}$ na equação (4.41) faz referência ao caso que estamos demonstrando: determinar os pontos umbílicos do tipo $D_{12}$.
} 


$$
\begin{aligned}
\left(\tau_{0}\right)_{12}(w) & =\left\{\frac{\left(k_{n}^{\perp}\right)^{\prime}(0)(\phi-1)^{2}\left[2 k_{n}^{\perp}(0)-\phi k_{n}^{\perp}(0)-\phi k_{n}(0)\right]}{\left[k_{n}(0)-k_{n}^{\perp}(0)\right]^{3}}\right\} w- \\
& -\frac{\left(k_{n}^{\perp}\right)^{\prime}(0) \phi^{\prime}(\phi-1)}{2\left[k_{n}(0)-k_{n}^{\perp}(0)\right]^{4}}\left\{\left(k_{n}^{\perp}(0)\right)^{2}\left[34 \phi^{2}-91 \phi+69\right]+\right. \\
& \left.+2 k_{n}(0) k_{n}^{\perp}(0)\left[19 \phi^{2}-33 \phi+2\right]+k_{n}^{2}(0)(13 \phi-1)\right\} w^{2}+\cdots
\end{aligned}
$$

Falta verificar a condição de transversalidade, $T$, ao longo destes pontos. Faremos isso no espaço de parâmetros $\varepsilon$ e $\tau_{0}$.

Seja $J_{1}$ a matriz jacobiana

$$
J_{1}:=\frac{\partial\left(\varepsilon, \tau_{0}\right)}{\partial(u, w)}=\left[\begin{array}{cc}
\frac{\partial \varepsilon}{\partial u}(u, w) & \frac{\partial \varepsilon}{\partial w}(u, w) \\
\frac{\partial \tau_{0}}{\partial u}(u, w) & \frac{\partial \tau_{0}}{\partial w}(u, w)
\end{array}\right]
$$

onde as expressões de $\varepsilon=\varepsilon(u, w)$ e $\tau_{0}=\tau_{0}(u, w)$ são dadas, respectivamente, por 4.28 e 4.29 .

Para $w$ próximo de 0 , concluímos que $\operatorname{det}(J)$ é não nulo. De fato,

$$
\begin{aligned}
\operatorname{det}\left(J_{1}\right)= & \frac{1}{\left(\tau_{g}^{\prime \prime}(0)\right)^{2}\left[k_{n}(0)-k_{n}^{\perp}(0)\right]^{6}\left[\phi k_{n}^{\perp}(0)-k_{n}(0)\right]}\{w(\cdots)+ \\
& \left.+(\phi-1)^{3}\left[\tau_{g}^{\prime \prime}(0)\left(k_{n}^{\perp}\right)^{\prime}(0)\left(k_{n}-k_{n}^{\perp}\right)^{\prime}(0)\right]+(\phi-1)^{4}(\cdots)\right\} .
\end{aligned}
$$

Pelas hipóteses do teorema, concluímos que $\operatorname{det}\left(J_{1}\right) \neq 0$.

(4) As expressões paramétricas de pontos umbílicos do tipo $D_{23}$ e do tipo $D_{12}$ dadas, respectivamente, pelas equações 4.32 e 4.42 coincidem somente quando $w=0$. De fato, observemos, por exemplo, que

$$
\begin{gathered}
\varepsilon_{12}(w)=\varepsilon_{23}(w) \Longleftrightarrow \varepsilon_{12}(w)-\varepsilon_{23}(w)=0 \Longleftrightarrow \\
\Longleftrightarrow \frac{9 w^{2} \phi^{\prime}(w)\left(k_{n}^{\perp}\right)^{\prime \prime}(0)[\phi(w)-1]^{2}\left[k_{n}^{\prime}(0)-\left(k_{n}^{\perp}\right)^{\prime}(0)\right]}{\tau_{g}^{\prime \prime}(0)\left[k_{n}(0)-k_{n}^{\perp}(0)\right]^{4}}=0 .
\end{gathered}
$$

Pela hipótese do teorema, sabemos que $k_{n}(0) \neq k_{n}^{\perp}(0), \quad k_{n}^{\prime}(0) \neq\left(k_{n}^{\perp}\right)^{\prime}(0)$ e que $\left(k_{n}^{\perp}\right)^{\prime \prime}(0)$ e $\tau_{g}^{\prime \prime}(0)$ são não nulos. Além disso, pelas propriedades da função de transição $\phi$ temos $\phi^{\prime}(w)<0$ e $[\phi(w)-1]^{2}>0$, pois $\phi$ é decrescente.

Consequentemente, a equação 4.45 só é satisfeita se $w=0$. Observe, também, que 
$\left(\tau_{0}\right)_{12}(w)=\left(\tau_{0}\right)_{23}(w)$ para $w=0$.

Portanto, supondo as hipóteses do teorema, usando a parametrização apresentada em 4.20 da superfície regularizada $\mathbf{S}_{\varepsilon, \mu}, \varepsilon, \mu>0$ suficientemente pequenos e as expressões de $L, M$ e $N$ definidas em 4.24 na carta $(u, w)$ (sendo $\left.w=v / \varepsilon^{2}\right)$ determinamos, no item (1) da demonstração deste teorema, pontos umbílicos sobre os quais a condição de transversalidade foi desrespeitada. Para decidir se os pontos umbílicos em (1) são do tipo $D_{23}$ verificamos, no item (2), que ao longo destes pontos, o contato entre $M(u, w, \varepsilon)=0$ e $N(u, w, \varepsilon)=0$ é quadrático (condição de Whitney). No item (3) encontramos pontos umbílicos sobre os quais a condição do discriminante foi vulnerada e vimos que nesses pontos o contato entre $M(u, w, \varepsilon)=0$ e $N(u, w, \varepsilon)=0$ é transversal, isto é, os pontos umbílicos são não-Darbouxianos do tipo $D_{12}$. No item (4) observamos que, no espaço de parâmetros $\varepsilon$ e $\tau_{0}$, as curvas $D_{23}$ e $D_{12}$ passam pela origem e não se interceptam em uma vizinhança dela, completando a demostração do teorema. 


\subsection{Apêndice}

Neste apêndice vamos apresentar um exemplo de uma função de transição. Esta função foi utilizada por Garcia e Sotomayor em [5].

A função de transição $\phi: \mathbb{R} \longrightarrow \mathbb{R}$, definida abaixo, é decrescente no intervalo $[0, \mu]$, $\phi^{(n)}(0)=\phi^{(n)}(\mu)=0$, para todo $n \in \mathbb{N}, \phi(v)=1$, para $v \leq 0$ e $\phi(v)=0$, para $v \geq \mu$ $\left(\right.$ onde $\left.\phi^{(n)}=\frac{d^{n} \phi}{d v^{n}}\right)$. Veja figura 4.9

Seja

$$
h(v)=\left\{\begin{array}{ll}
0, & \text { se } v \leq 0 \\
e^{-a / v}, & \text { se } 0<v \leq \mu
\end{array},\right.
$$

onde $a>0$ é uma constante.

Considere a função $\phi$ como sendo definida por

$$
\phi(v)=\frac{h(\mu-v)}{h(v)+h(\mu-v)} .
$$

Observe que $\phi$ definida em 4.46 satisfaz:

$* \phi(v)=1$, para todo $v \leq 0$.

De fato, $\phi(v)=\frac{h(\mu-v)}{h(v)+h(\mu-v)}=1$, pois $h(v)=0$, para $v \leq 0$

$* \phi(v)=0$, para todo $v \geq \mu$.

De fato, $\phi(v)=\frac{h(\mu-v)}{h(v)+h(\mu-v)}=0$, pois $h(\mu-v)=0$, para $v \geq \mu$;

$* \phi(v)$ é decrescente em $(0, \mu)$.

$$
\text { De fato, } \phi^{\prime}(v)=-\frac{a e^{\frac{a \mu}{(v-\mu) v}}\left[v^{2}+(\mu-v)^{2}\right]}{v^{2}(\mu-v)^{2}\left[e^{-\frac{a}{v}}+e^{\frac{a}{v-\mu}}\right]^{2}}<0 \text {, para } a>0 \text { e } 0<v<\mu \text {. }
$$

Além disso, das propriedades da função exponencial na base e podemos concluir que $\phi$ tem todas as derivadas iguais a zero em $v=0$ e $v=\mu$ e $\phi_{v v}(v)<0$, para $v$ próximo de zero e $a>0$ suficientemente pequeno.

Portanto, $\phi$ definida em 4.46 é uma função de transição.

Veja na figura 4.9, abaixo, um esboço do gráfico de $\phi$ para valores de $\mu$ e $a>0$ suficientemente pequenos. 


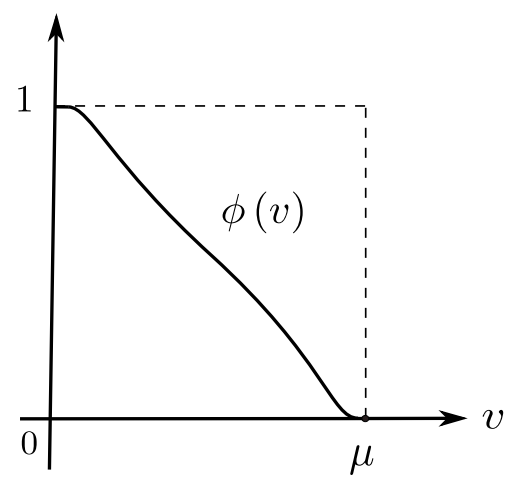

Figura 4.9: Gráfico da função de transição $\phi$, para $a>0$ e $\mu>0$ suficientemente pequenos. 


\section{Conclusões}

Neste trabalho retomamos a sequência de contribuições encontradas nas publicações de Gutierrez e Sotomayor [7] e [8], Gutierrez, Garcia e Sotomayor [9] e Garcia e Sotomayor [5], particularmente esta última na qual são abordadas questões sobre os efeitos da descontinuidade das superfícies, representada pela quebra da regularidade ou suavidade ao longo de uma curva, na configuração principal das mesmas. Desta forma, este estudo se insere na linha do trabalho clássico de Filippov [3] para o retrato de fase das equações diferenciais descontínuas em geral, aspectos que foram reformulados e estendidos por Sotomayor e Teixeira [12] e Sotomayor e Machado [11], usando o método da regularização e comparando as equações diferenciais resultantes com os sistemas estruturalmente estáveis.

Assim nosso trabalho se conecta com as contribuições pioneiras de Andronov - Pontrjagin e Peixoto sobre a estabilidade estrutural das equações diferenciais e sistemas dinâmicos e com as bifurcações.

Resumimos a seguir em forma circunstanciada os resultados de nosso estudo.

Sendo $\left(\mathbf{S}^{+}, \mathbf{B}\right)$ e $\left(\mathbf{S}^{-}, \mathbf{B}\right)$ duas superfícies imersas em $\mathbb{R}^{3}$ e $\mathbf{B}$ a linha de descontinuidade separando $\mathbf{S}^{+}$e $\mathbf{S}^{-}$, considere $\mathbf{S}=\mathbf{S}^{+} \cup \mathbf{B} \cup \mathbf{S}^{-}$, a superfície seccionalmente suave obtida pela justaposição de $\mathbf{S}^{+}$e $\mathbf{S}^{-}$ao longo do bordo comum $\mathbf{B}$. Estudamos os pontos nos quais as linhas de curvatura principal de ambas as superfícies $\mathbf{S}^{+}$e $\mathbf{S}^{-}$têm contato quadrático ou cruzam transversalmente a curva de descontinuidade, representada pela curva bordo B. Concluímos com os teoremas 2.2.6, 2.2.10, 2.2.11, 3.2.4 e 3.3.1 que, de tais pontos sobre $\mathbf{B}$ emergem pontos umbílicos Darbouxianos dos tipos $D_{1}$ e $D_{3}$. O ponto umbílico Darbouxiano do tipo $D_{2}$ não surgiu neste processo de regularização. Por outro lado, sendo $\mathbf{S}$ uma superfície imersa em $\mathbb{R}^{3}$ com bordo $\mathbf{B}$ para a qual o contato da linha de curvatura principal com B é cúbico, concluímos com o teorema 4.3 .4 que os pontos umbílicos Darbouxianos dos tipos $D_{1}, D_{2}$ e $D_{3}$ e também os tipos não Darbouxianos $D_{23}$ e $D_{12}$ surgem sobre a superfície engrossada e regularizada.

Acreditamos que as questões locais abordadas nesta tese possam servir de ponto de apoio para estudos geométricos e analíticos mais profundos e globais focalizando a relação entre a regularização das superfícies seccionalmente suaves e suas configurações principais. 


\section{Referências Bibliográficas}

[1] Bruce, J., And Fidal, D. On binary differential equations and umbilics. Proceedings of the Royal Society of Edinburgh, 111A (1989), 147-168.

[2] Darboux, G. Sur la forme des lignes de courbure dans la voisinage d'un ombilic. Gauthier Villars IV, Note 07, Leçons sur la théorie de surfaces (1896).

[3] Filippov, A. F. Differential Equations with Discontinuous Righthand Sides. Kluwer Academic Press, 1988.

[4] Garcia, R., And Sotomayor, J. Structural stability of parabolic points and periodic asymptotic lines. Matemática Contemporânea, Soc. Bras. Matemática 12 (1997), 83-102.

[5] Garcia, R., And Sotomayor, J. Umbilic and tangential singularities on configurations of principal curvature lines. Anais da Academia Brasileira de Ciências 74, 1 (2002), 1-17.

[6] Garcia, R., and Sotomayor, J. Differential Equations of Classical Geometry, a Qualitative Theory. $27^{\text {th }}$ Colóquio Brasileiro de Matemática. IMPA, Rio de Janeiro, 2009.

[7] Gutierrez, C., And Sotomayor, J. Structural stable configurations of lines of principal curvature. Asterisque, 98-99 (1982), 185-215.

[8] Gutierrez, C., And Sotomayor, J. Lines of Curvature and Umbilic Points on Surfaces. $18^{\text {th }}$ Colóquio Brasileiro de Matemática. IMPA, Rio de Janeiro, 1991.

[9] Gutierrez, C., Sotomayor, J., and Garcia, R. Bifurcations of umbilic points and related principal cycles. Journal of Dynamics and Differential Equations 16, 2 (2004), 321-346.

[10] Monge, G. Sur les lignes de courbure de la surface de l'ellipsoide. Journ. de l' Ecole Polytech. II cah. (1796), 145-165.

[11] Sotomayor, J., And Machado, A. L. F. Structurally stable discontinuous vector fields in the plane. Qualitative Theory of Dynamical Systems 3 (2002), 227-250. 
[12] Sotomayor, J., And Teixeira, M. A. Regularization of discontinuous vector fields. World Scientific Publishing River Edge (1998), 207-223.

[13] SpIvak, M. A Comprehensive Introduction to Differential Geometry, vol. III. Berkeley: Publish or Perish, Houston, Texas, 1999.

[14] Whitney, H. On singularities of mappings of euclidean spaces. i. mappings of the plane into the plane. Annals of Mathematics 62, 3 (1955), 374-410. 


\section{Índice Remissivo}

Bordo

orientação externa do, 74

superfícies com, 56, 74

Bordo comum $\mathbf{B}_{\varepsilon, \pm}, 75$

Bordo comum $\boldsymbol{\delta}, 15,61$

Campo de Lie-Cartan, 4

Carta de Monge, 5

Coeficientes Forma Fundamental

Primeira, 35, 63, 79, 80, 90

Segunda, 36, 63, 79, 90

Configuração principal, 3

da superfície $\mathbf{S}=\mathbf{S}^{+} \cup \mathbf{B} \cup \mathbf{S}^{-}, 61$

da superfície $\mathbf{S}_{\varepsilon}=\mathbf{S}_{\varepsilon,+} \cup \mathbf{T}_{\varepsilon} \cup \mathbf{S}_{\varepsilon,-}$, 81

do $\operatorname{par}(\mathbf{S}, \mathbf{B}), 74$

Conjunto característico, 74

Curvatura

geodésica, 58

normal, 2, 58

Curvaturas principais, 2

Direções principais, 2

Engrossamento de uma superfície em $\mathbb{R}^{3}$ com bordo, 75

Equação diferencial das linhas de curvatura, 3. 63, 91

Formas fundamentais, 2

Função de transição, $33,62,88$

Linhas de curvatura principal, 3

Parâmetro de regularização, 33

Ponto de

costura, 24, 25 deslizamento, 23

dobra, 32

transição, 24

Ponto umbílico, 2

Ponto umbílico $D_{12}, 7$

Ponto umbílico $D_{23}, 10$

Ponto umbílico Darbouxiano, 4, 6

Referencial de Darboux associado a uma curva, $58,76,85$

Região de regularização, 34

Regularização, 33, 62, 88

Separatrizes umbílicas, 6, 8

Superfície

canal, 75

de Lie-Cartan, 4

envelope de, 74

regularizada, 33

Torção geodésica, 58

Vetor normal, 35 